\title{
Development of Centroid Based Metrics to Provide Personalized Monitoring and Assessment of Gait
}

\author{
A Dissertation Presented to \\ The Faculty of the Graduate School \\ University of Missouri-Columbia \\ in Partial Fulfillment \\ of the Requirements for the Degree \\ Doctor of Philosophy \\ by \\ ROBERT STUART WALLACE, III \\ M.S. Engineering Management, University of South Florida 2001 \\ M.S. Biomedical Engineering, Boston University, 1991 \\ B.S. Biomedical Engineering, Boston University, 1987
}

Dr. Marjorie Skubic, Dissertation Supervisor

DECEMBER 15, 2017 
The undersigned, appointed by the dean of the Graduate School, have examined the dissertation entitled

\section{Development of Centroid Based Metrics to Provide Personalized Monitoring and Assessment} of Gait

\section{Presented by Robert S. Wallace, III}

A candidate for the degree of doctor of philosophy, and hereby certify that, in their opinion, it is worthy of acceptance.

Professor Marjorie Skubic

Professor Marilyn Rantz

Professor Mihail Popescu

Professor Jianlin Chen

Professor Yunxin Zhao 
To: Audrey, Aidan, and Nolan

...26 years later, the miracles keep occurring! 


\section{ACKNOWLEDGEMENTS}

I would first like to thank Dr. Marjorie Skubic for her guidance, support, and patience during those times when life interrupted my progress. Particularly the last several years as I have been a remote student. From my original plan to contribute to the CERT using my experiences as an embedded software engineer, to a stint working with image processing, and finally to analyzing gait using centroid based metrics, you have helped me to find something new and different that excited me. Thank you!

I would also like to thank Dr. Rantz, Dr. Popescu, Dr. Cheng, and Dr. Zhao for reviewing this document and offering suggestions. Of course, your help has not been limited just to this document and the research it represents, but includes helping me learn, both in the classroom and in the laboratory, machine learning, fall risk assessments, and many other concepts too numerous to mention here.

I also would like to thank Dr. Carmen Abbott for the help you have given me through each of the papers and weekly lab meetings back when I was still in Columbia. It really excited me when, after describing some of my results, to hear you say that it made sense clinically.

I want to thank my wife Audrey and my sons Aidan and Nolan. Audrey, despite juggling medical school and residency, you always provided me with the time I needed to get my work done. Without your support and encouragement, it would have been much harder. Aidan, you were 4 when I started this journey, and you are almost 12 . Thank you for your understanding on those occasions over the years when I had to "do my homework" instead of going out to play. And to Nolan, thank you for joining us nearly 2 years ago. The house was just getting too quiet and you have done your best to correct it. Lastly, I would like to acknowledge the National Science Foundation for funding this research. 


\section{TABLE OF CONTENTS}

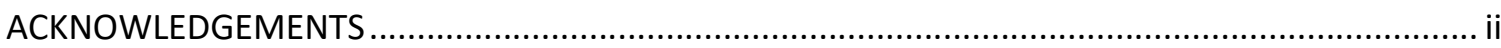

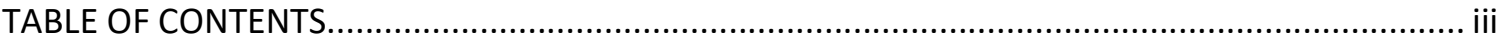

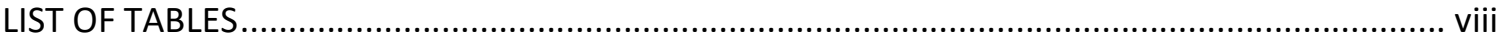

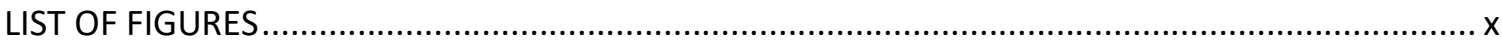

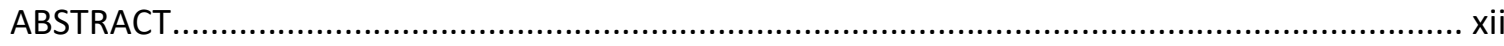

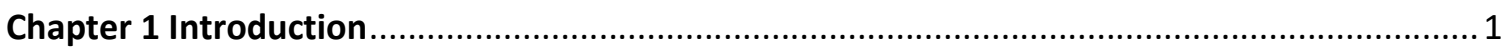

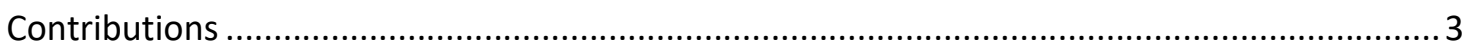

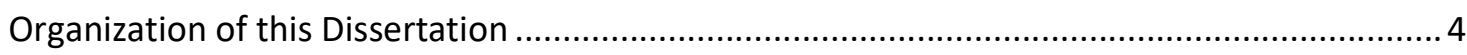

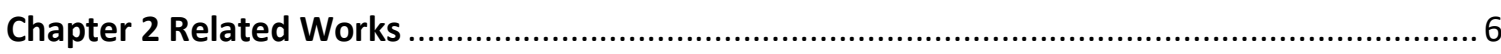

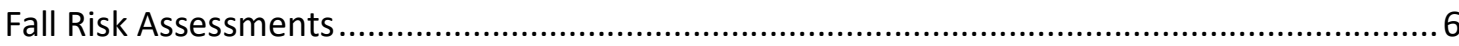

Balance

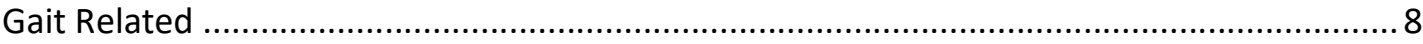

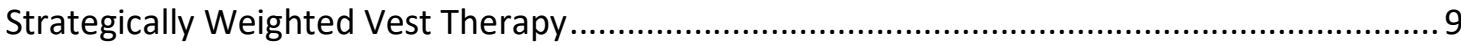

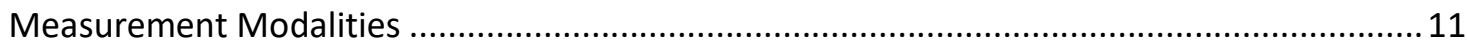

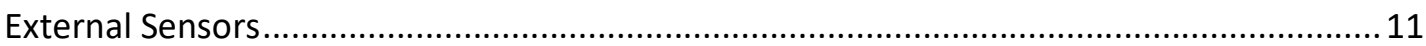

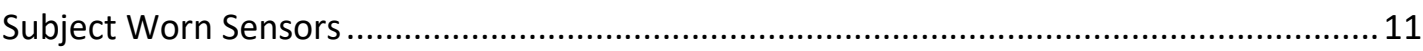

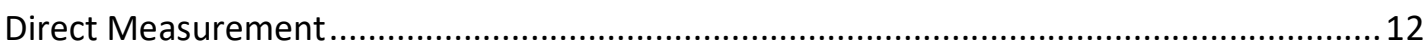

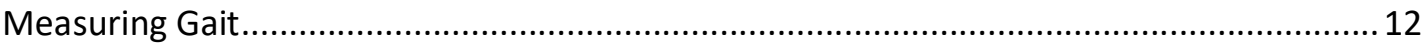

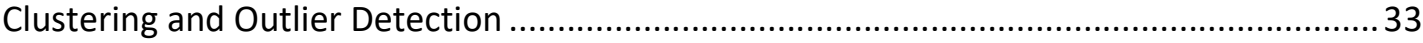

Chapter 3 Metrics from In-Home Sensor Data to Assess the Effect of Clinical Therapies .......... 39

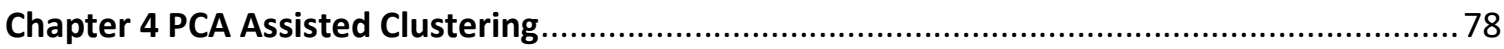

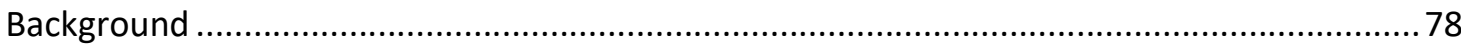

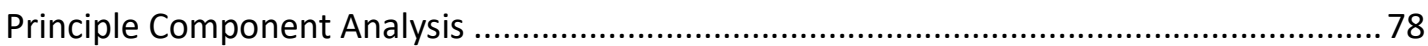

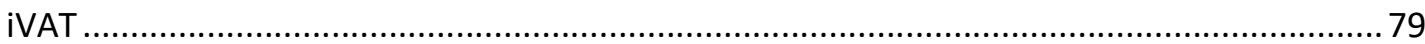

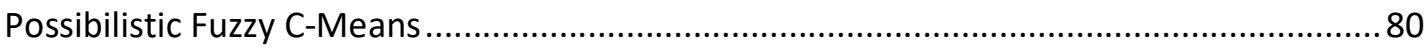

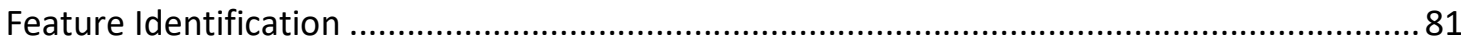

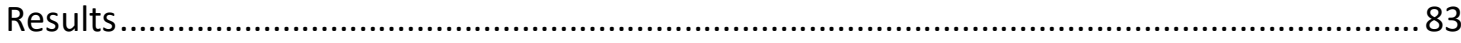

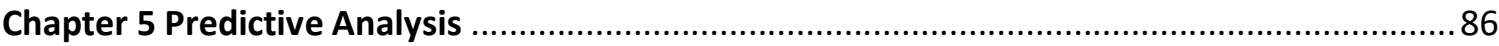

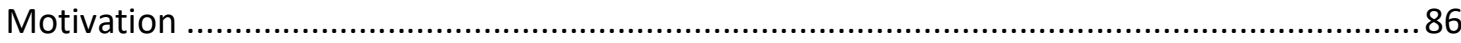

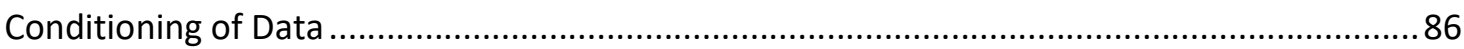

Outlier Detection using One Class Possibilistic C-Means (OCPCM) ...................................... 88

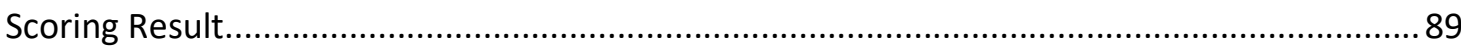




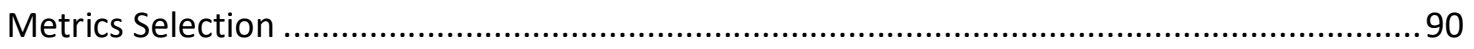

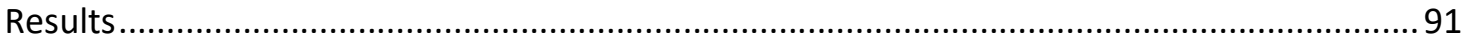

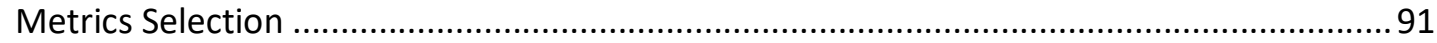

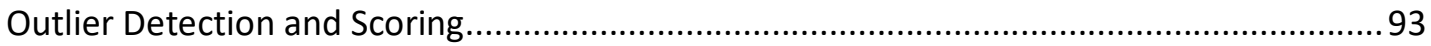

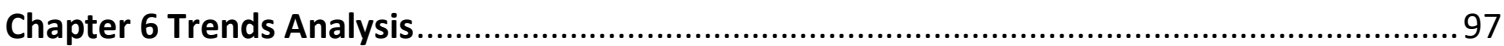

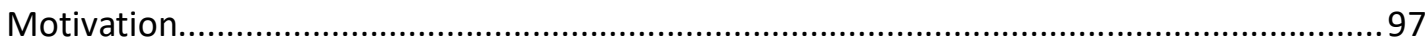

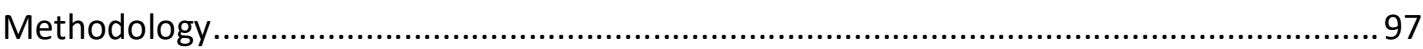

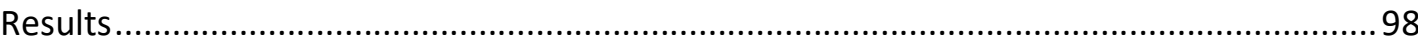

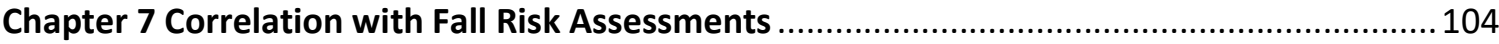

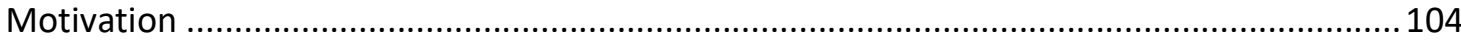

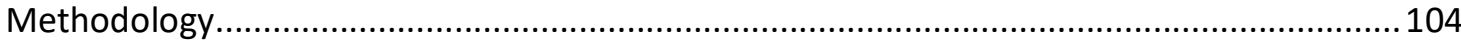

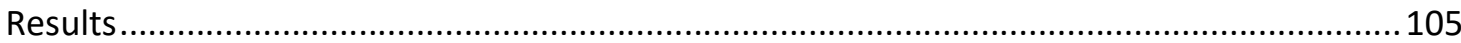

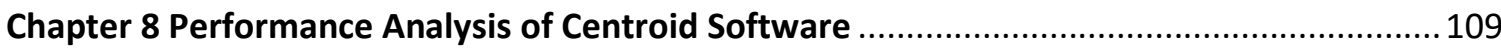

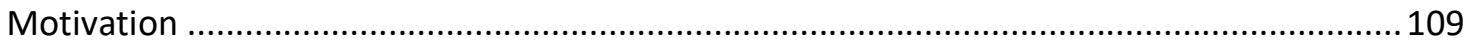

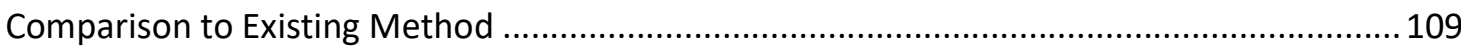

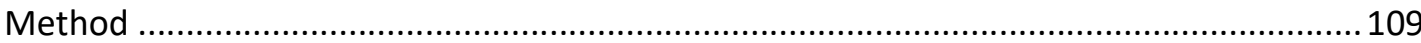

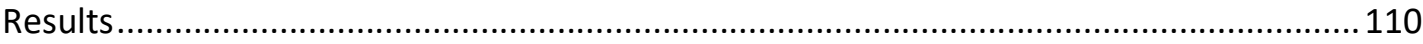

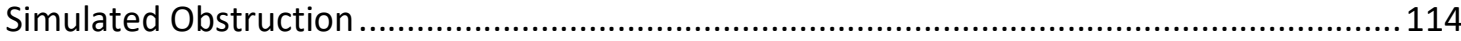

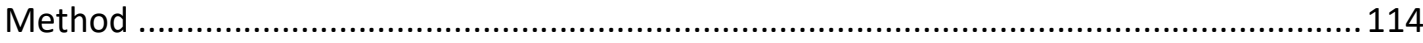

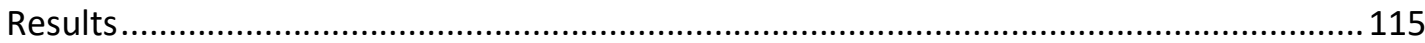

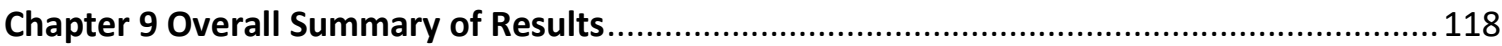

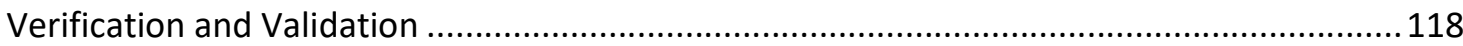

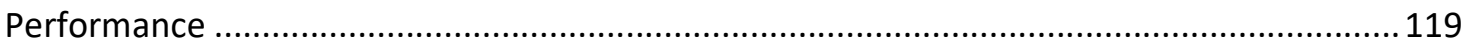

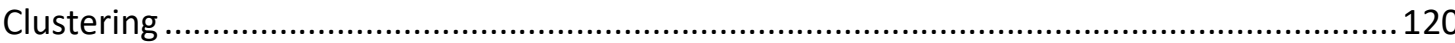

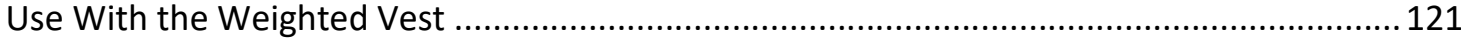

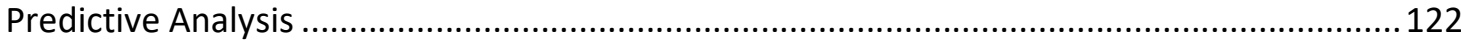

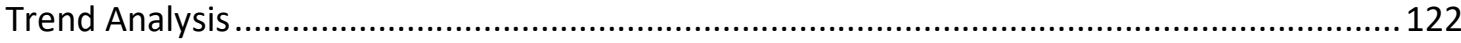

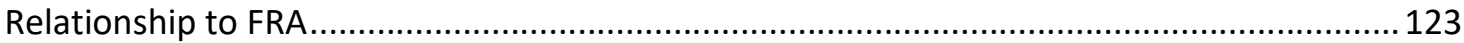

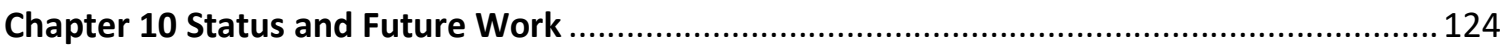

Appendix I: Summary of Current Status of Proposed Tasks ................................................. 128

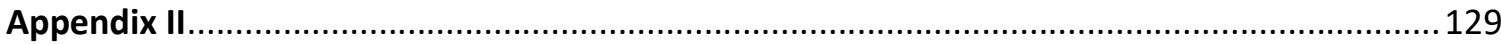

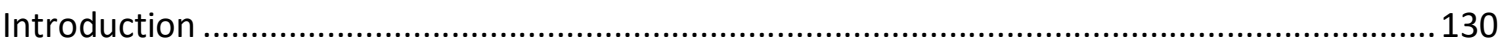

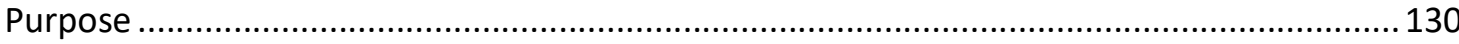


Scope

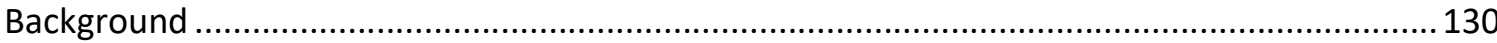

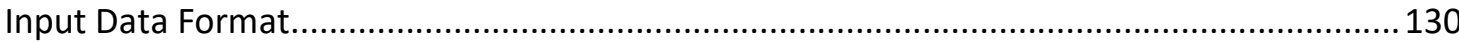

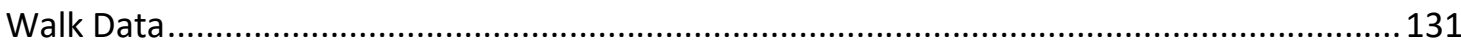

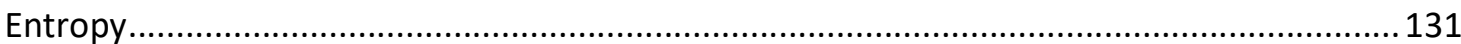

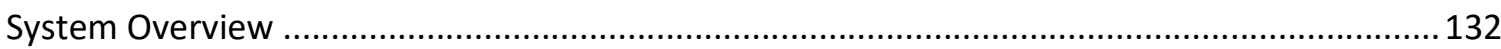

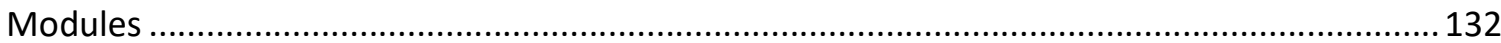

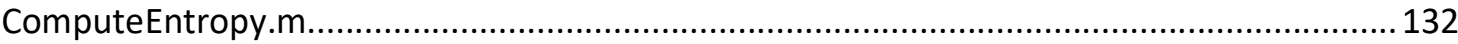

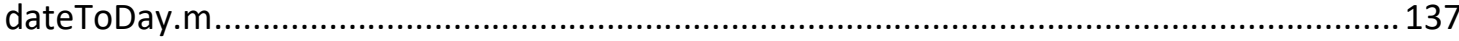

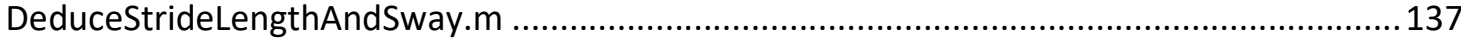

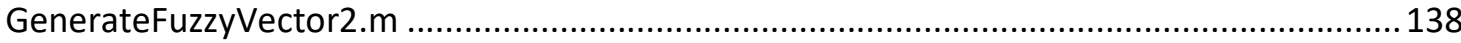

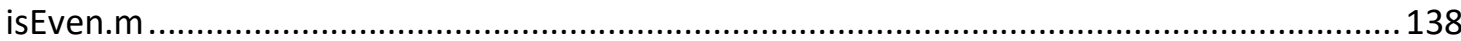

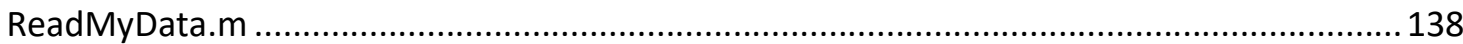

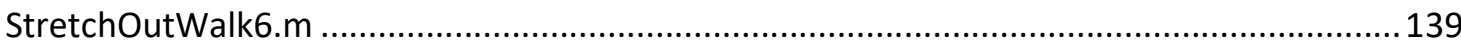

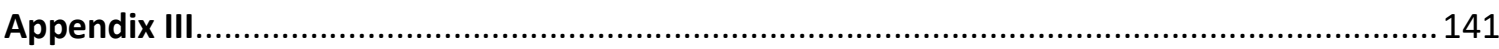

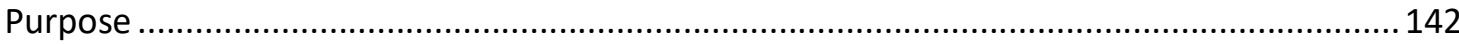

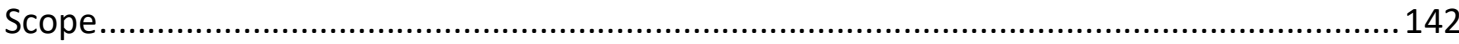

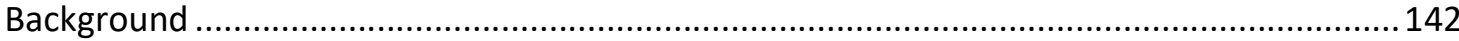

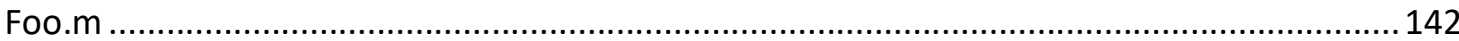

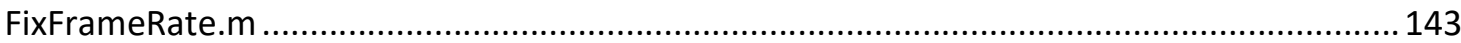

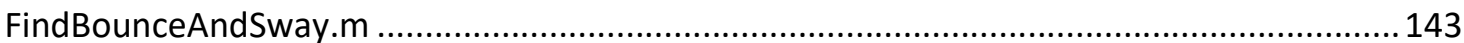

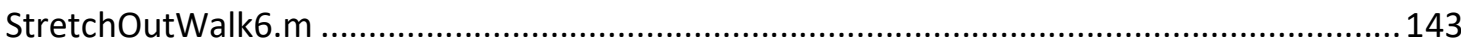

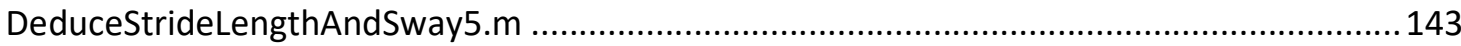

Appendix IV - SDD for Resident Validation Component ..................................................... 144

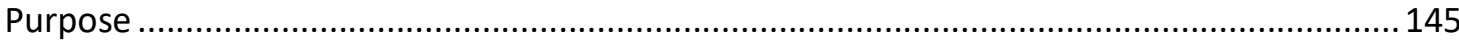

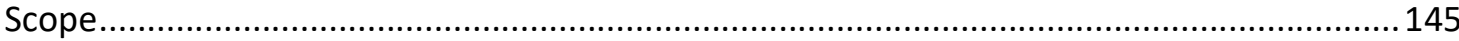

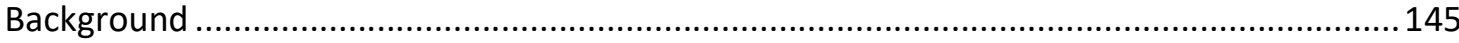

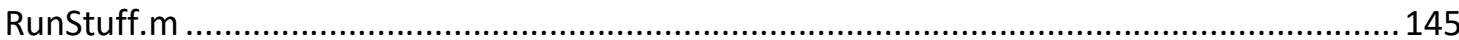

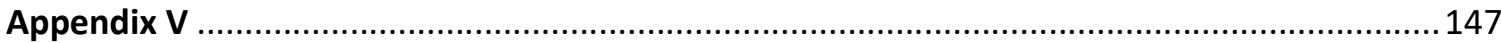

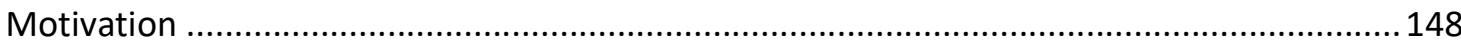

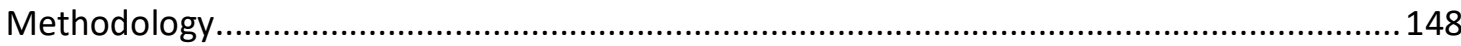

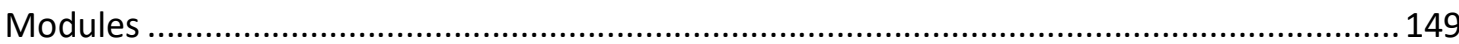

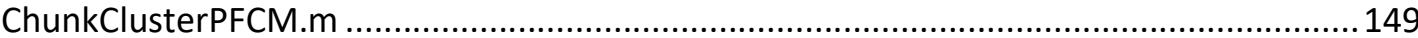




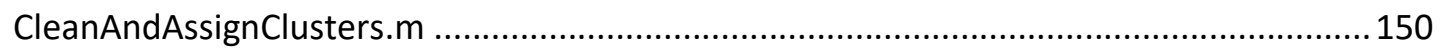

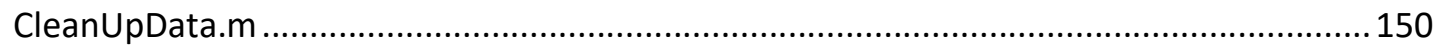

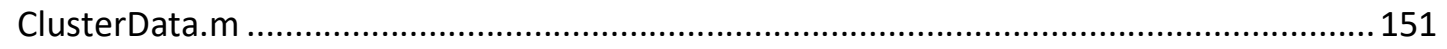

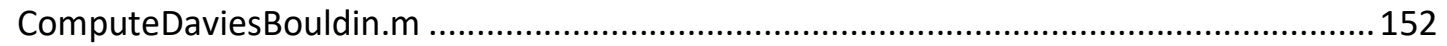

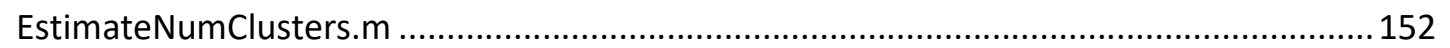

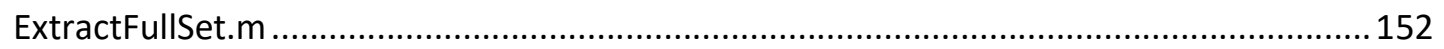

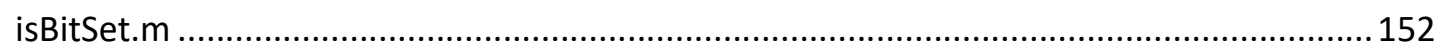

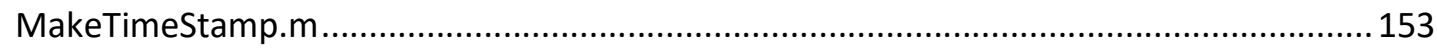

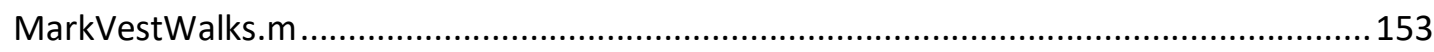

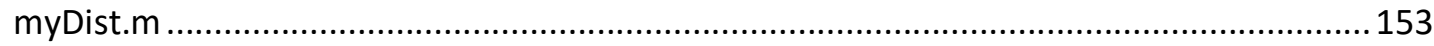

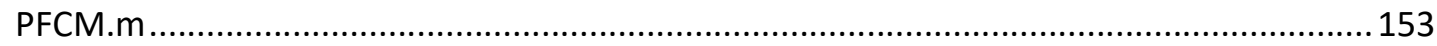

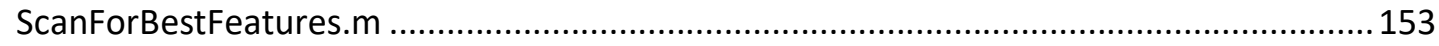

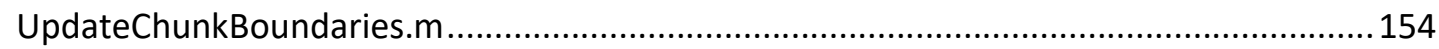

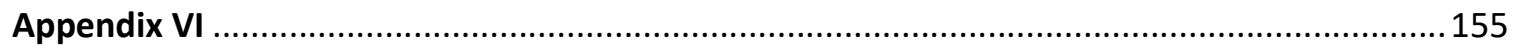

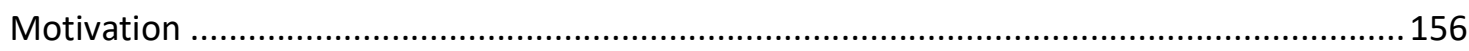

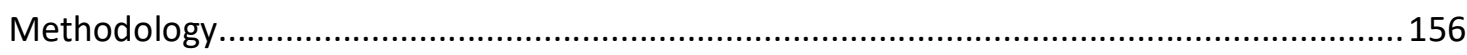

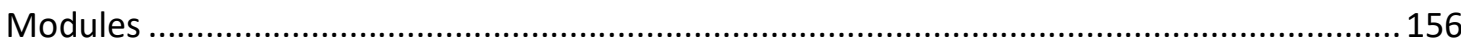

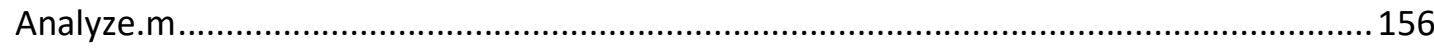

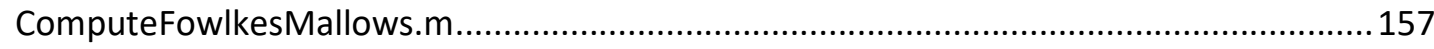

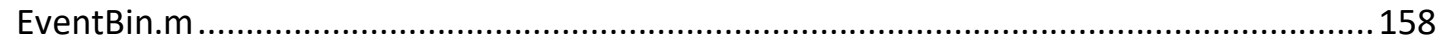

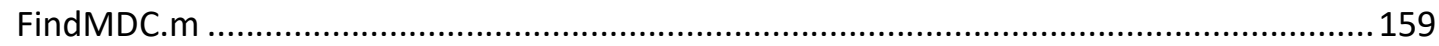

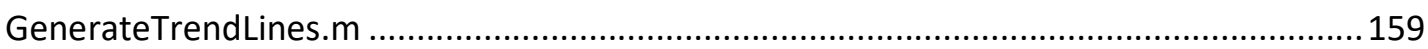

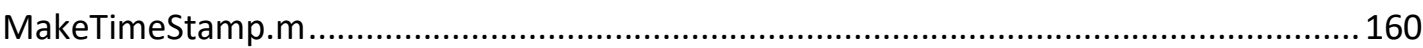

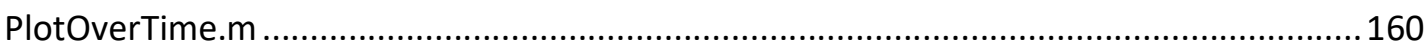

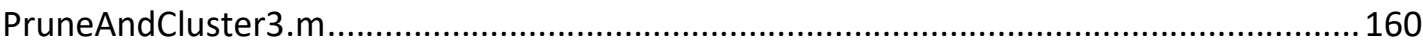

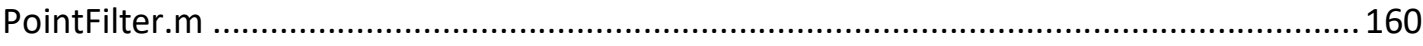

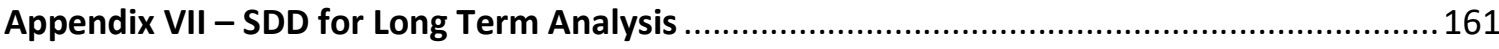

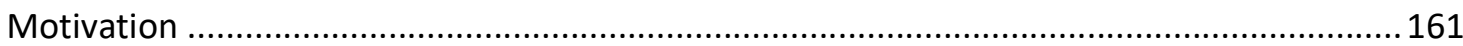

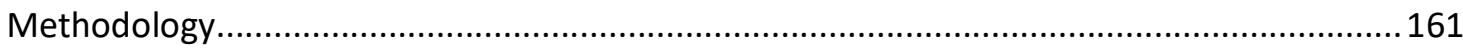

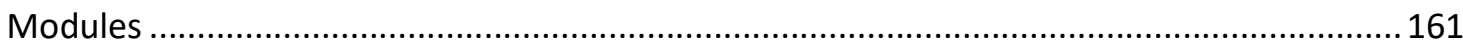

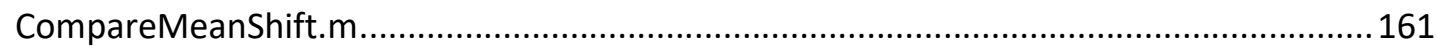

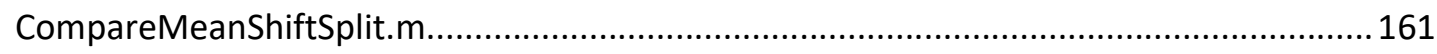

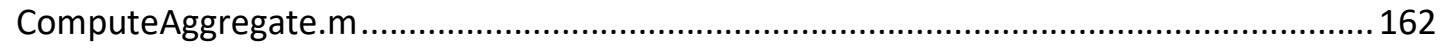




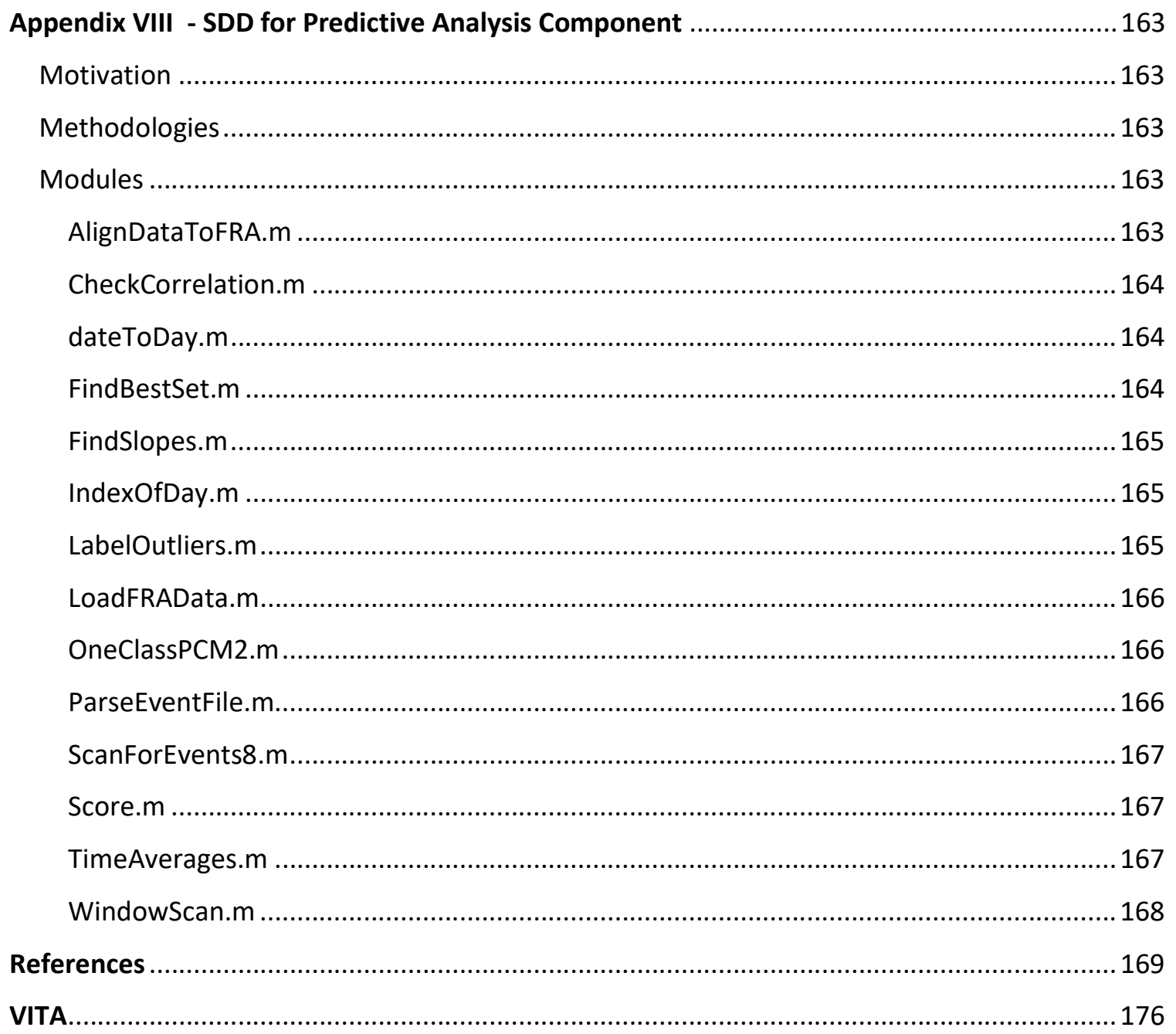




\section{LIST OF TABLES}

Table 2-1: Validation results from accelerometer derived gait parameters ...............................16

Table 2-2: Results from McDonough et al. comparing paper-pencil and video methods............19

Table 2-3: Results from Miaou et al. [64]. Table II shows a truth table for fall recognition.........20

Table 2-4: Comparison of joint angles computed by the proposed algorithm ............................24

Table 2-5: Results comparing the Kinect based motion capture system to the Vicon .................25

Table 2-6: Differences in Walking Speed, Stride Time, and Stride Length..................................29

Table 3-1: Results from validation of proposed algorithm using in-home data ...........................60

Table 3-2: Correlation Between Proposed Metrics and FRA for Subject \#1...............................61

Table 3-3: Subjects With Strong Correlation Between Proposed Metrics and FRAs ....................62

Table 3-4: Correlations Between Proposed Metrics and FRAs With Aggregated Data...............63

Table 3-5: Window Size for Weighted Vest Analysis ...............................................................64

Table 3-6: Short Term Impact of the Weighted Vest Therapy on the Proposed Metrics .............65

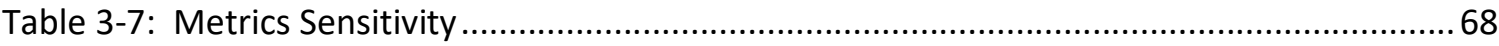

Table 4-1: Metrics Used for Resident Identification Clustering .................................................82

Table 5-1: Classification criteria for scoring outliers against clinical events...............................89

Table 5-2: Chart of the metrics used in the optimal outlier detection.....................................91

Table 5-3: Scores for eleven residents. M/F shows resident's gender. ....................................93

Table 5-4: Scores for eleven residents using a fixed set of 16 metrics......................................94

Table 6-1 Percentage of clinical events corresponding to outliers for each metric....................99

Table 6-2: Qualitative changes in 9 metrics over 14,42 , and 70 day periods .........................100

Table 6-3: Relationship between trends in metrics 30 days prior to, and 30 days following.....102

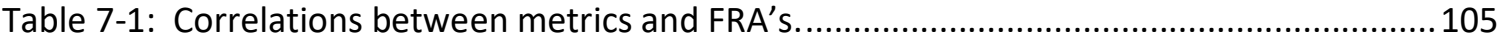

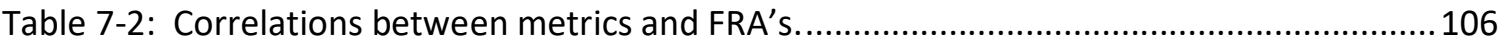


Table 7-3: Correlations between metrics and FRA's.

Table 8-1: Results from comparing existing method results to centroid based method........... 110

Table 8-2: Results from comparing existing method results to centroid based method........... 115 


\section{LIST OF FIGURES}

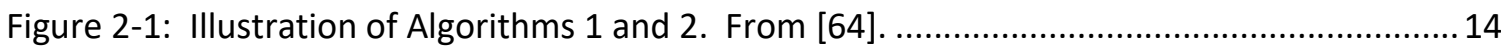

Figure 2-2: Components of the "GaitShoe" device. ............................................................ 18

Figure 2-3: Location of joints (red dots) of the subject taken using a ToF camera. .....................2 21

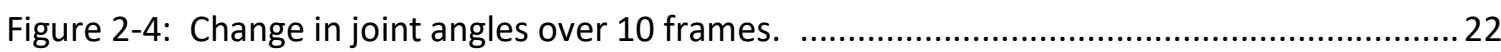

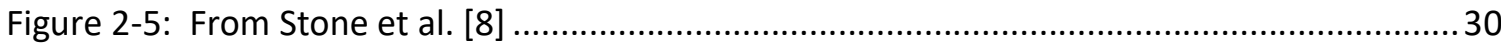

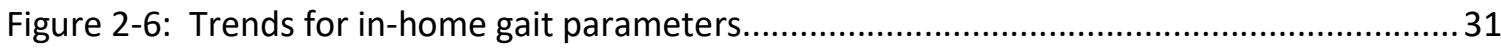

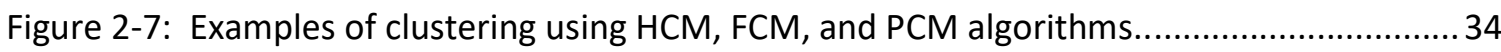

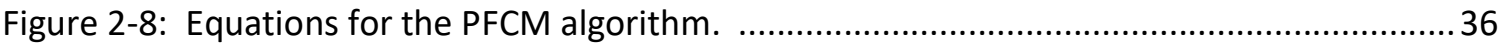

Figure 2-9: Graph of movement of the cluster center during a One Class PCM computation.....38

Figure 3-1: Diagram of the process used to generate the $(\delta x, \delta y$, and $\delta z)$ error signals ............. 48

Figure 3-2: An example of strategically weighted vest used in this study................................56

Figure 3-3: Plot showing Entropy in the XY direction for subject \#3004 ..................................57

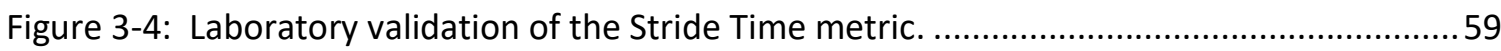

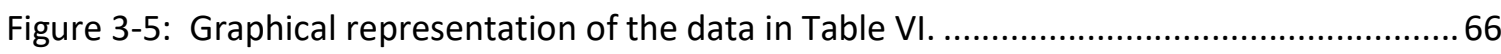

Figure 4-2 Example of an iVAT image for a two resident apartment. ........................................80

Figure 4-3: Two examples of an iVAT image for a single resident apartment............................ 82

Figure 4-4: Clustering attempt using the step length metric for a two resident apartment........83

Figure 4-5: Progression of two week chunks clustered using PFCM with PCA transform............84

Figure 5-1: Frequency of inclusion in optimal set for each resident. .......................................92

Figure 5-2: Comparison between the personalized set of features and a fixed set ....................94

Figure 8-1: PDF's for Stride Length (left), Stride Time (center), and Average Speed (right) .......112

Figure 8-2: PDF's for Stride Length (left), Stride Time (center), and Average Speed (right) ...... 112

Figure 8-3: PDF's for Stride Length (left), Stride Time (center), and Average Speed (right) ...... 113 
Figure 8-4: PDF's for Stride Length (left), Stride Time (center), and Average Speed (right) ...... 113

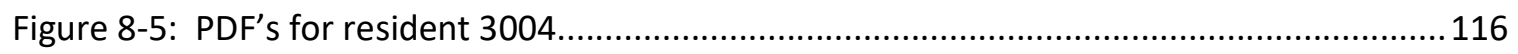

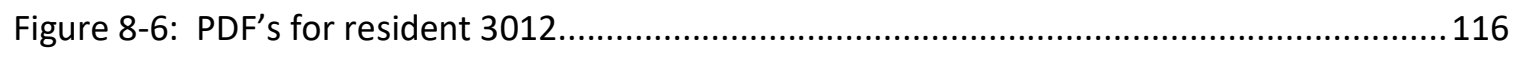

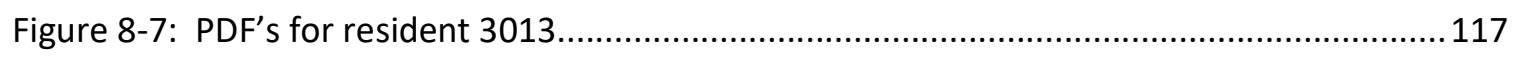

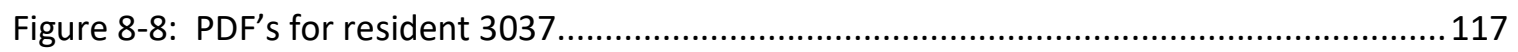




\begin{abstract}
A methodology is presented to characterize a subject's ability to ambulate using various metrics generated from the movement of the subject's centroid as detected using an inexpensive depth camera system. Metrics have been developed focusing on three major categories of motion. The first, and most basic, class measures characteristics of movement in the direction of motion, laterally, and vertically. The second class focuses on measuring the entropy that exists in the subject's walk. The third class uses periodicity in the subject's motion to deduce temporal gait parameters including stride length, and step times on the left and right side. As each patient is unique with different histories, disease progression, and overall state, metrics and the associated analysis approaches integrate a personalized approach to selecting and using metrics.
\end{abstract}

These stride time, stride length, and average speed metrics were then validated against both the Vicon ${ }^{\circledast}$ system and an established reference algorithm. From these metrics, a set of methodologies were developed to study short and long-term effects of therapies, significant changes in metrics due to clinical events, as well as the ability to predict potential clinical events by identifying outliers in long term trends.

These metrics and my analysis approach were then tested using a group of subjects undergoing therapy using strategic weighted vests. The ability of the metrics to show changes in the subject's ambulation when the vest is either put on, or taken off was examined. Results show sufficient sensitivity to detect changes when the vest is donned and doffed. Interestingly, results also show that the effects of the vest are not seen immediately, but over 2-4 hours following donning. Results also demonstrate the ability, using the window size, to 
focus on the time required for the effects of each metric to change. Lastly, results show distinct results for each individual subject.

Additional studies were also done using subjects not undergoing the vest therapy to identify trends and outliers as portents of clinical events. Results show the ability to identify potential clinical events by identifying outliers in long term trends. Again, results are improved if the metrics used in the analysis are chosen specific to each subject.

The metrics are also compared against existing Fall Risk Assessments to validate their potential usefulness in monitoring subjects for changing risk of falls. While results show strong correlation to some FRA's, not every subject has the same relationships between metrics and FRA's suggesting a unique "fingerprint" of metrics associated with a subject's condition.

Lastly, the performance of these metrics was evaluated against a reference algorithm using in home data as well as against in home data into which a simulated obstruction was placed. In both cases, greater than $90 \%$ of the walks could produce a valid set of metrics and the simulated obstruction resulted in no significant changes in the examined metrics.

The metrics and methodologies presented here show a significant improvement in performance, a wider diversity of measurements, as well as the ability to measure both short term and long-term trends than currently existing approaches. 


\section{Chapter 1 Introduction}

More than one third of older adults fall each year[1]-[4]. Of these falls, $10 \%$ to $20 \%$ cause serious injuries. In the year 2000 alone, there were nearly 10,300 fatal and 2.6 million non-fatal falls in the United States costing $\$ 0.2$ million and $\$ 19$ million respectively [5]. Of these falls, about three percent resulted in a fracture of some kind [6].

Beyond the physical and immediate financial cost of falls, there are longer term costs, psychological, physical and financial, that result from falling. Howland reported that the fear of falling may, in fact, affect social interaction regardless of actual fall risk [7]. Nevitt found that approximately one quarter of falls cause the subject to limit their normal activities, usually because of injury, but often simply due to the fear of falling again [6]. Reducing the occurrence of falls, then, can go a long way in reducing the direct and indirect costs of falls. Not only will the patient's life be extended, and direct hospitalization costs be reduced, but more patients will be able to maintain their functional independence longer, delaying or eliminating their dependency on family, friends, or the healthcare system as a whole. This will lead to a higher quality of life for the patient.

The current standard of care involves assessment of the patient's condition and delivery of therapy in a clinical, laboratory, or possibly an assisted living facility where specialized health care practitioners periodically visit. In these settings, the practitioner will often utilize one or more Fall Risk Assessment Instruments (FRA's) to assess the subject's current condition. These FRA's, while very effective in assessing the patient's state, require a trained professional and, possibly, specialized equipment to administer properly. There are several reasons why this is not the ideal solution. Primary among these are the cost of a health care practitioner's time, the cost of equipment, and the inconvenience of visiting a doctor's or physical therapist's office periodically. 
Additionally, the behavior of the patient in these settings is different from their behavior at home. It is easy to imagine the case where a patient who fears interventions may take extra care than what is taken at home to improve their score. What is needed is a method of continuously monitoring the patient in familiar and comfortable settings with a system that can provide information about the patient's ambulation as they go about their daily business.

Numerous approaches to monitor a patient's ambulation have been devised using an array of different technologies. Many of these involve costly specialized equipment, such as sophisticated motion capture systems or pressure sensitive flooring. Others utilize sensors that must be worn by the subject like accelerometers, or other methods that present a financial or cognitive burden to the subject. Other research has been conducted on the use of visual monitoring of the subject's ambulation through different camera technologies. Other researchers in our lab have devised, using inexpensive depth camera systems such as the Microsoft Kinect, an approach where the subject's gait is monitored in real time by "watching" movement of the lower body / feet [8]. While this approach is very effective when the lower part of the body is visible, objects in an unstructured, cluttered residence can occlude the subject's feet and create challenges to properly determine gait parameters. To supplement and complement this approach, an approach allowing the measurement of stride time and step length, as well as other additional metrics, using centroid data could prove beneficial.

Monitoring the subject's centroid will remove the requirement that the subject's feet be visible to the camera. To a depth camera mounted near the ceiling "looking down" towards the subject, many occlusions caused by typical furniture would be below the subject's centroid. In the worst case, the centroid position can be estimated based upon the remainder of the subject's silhouette. 
Another focus recently has been the idea of precision medicine - medical interventions, diagnoses, and other care specifically tailored to the individual patient. Typical gait analysis, to date, has focused on a few highly effective metrics (walking speed, step length (right, left, total), and step time (right, left, and total)). For a given disease, different patients will present differently and show different disease progressions due to genetics, history, and environmental conditions. It makes sense, then, to use a broad selection of metrics to better characterize a person's gait.

To monitor the subject in this manner, it is necessary to develop a set of metrics that can be used to characterize the dynamics of gait and balance and can be monitored on a continuous basis to study ambulation over the patient's day-to-day activities. In this dissertation, I present a set of metrics, and methodologies to analyze both the short term and long-term trends of the monitored subjects. The metrics can be computed based upon the subject's centroid position and can be used to monitor and characterize the ambulation of the subject. A wide variety of metrics are developed as individuals will have different expressions of disease conditions or decline based upon genetic and environmental influences over their lifetimes. These metrics will be demonstrated on a cohort of residents at Tiger Place, an assisted living facility in Columbia, MO. A subset of these residents has undergone weighted vest therapy during the study period. The metrics have been used to characterize long term, short term, and predictive ability of this therapy. The second group consists of patients who are not undergoing a specific therapy but who have a history of clinical events (falls, Emergency Department visits, and hospitalizations) during the study period. These metrics have been used in this group to study long term and predictive ability for falls and other clinical events.

\section{Contributions}

This work presents several novel contributions to the field. These contributions include: 
1. A set of metrics that measure fundamental aspects of a subject's ambulation by monitoring the movement of the subject's centroid. Published in [9], [10]. (Chapter 3)

2. An algorithm that computes the average step time and average step length based upon the movement of the subject's centroid. Published in [9], [10]. (Chapter 3)

3. A Short-Term analysis technique that can be used to identify changes in metric values coincident with the application of therapies. Published in [9], [10]. (Chapter 3).

4. An improved clustering algorithm, using Principle Component Analysis, that more effectively separates the data from multiple resident apartments. Published in [11]. (Chapter 4)

5. A predictive analysis technique which measures metrics over the long term with various subjects and conditions with the goal of demonstrating the ability of these metrics to identify changes. Published in [11]. (Chapter 5)

6. A Long-Term analysis technique that can be used to identify long term changes in metric values as the result of particular therapies or events. Published in [11]. (Chapter 6)

7. Demonstration of the relationship between these metrics and established fall risk assessments. Published in [9], [10], [11]. (Chapter 7)

8. Demonstration of the insensitivity of centroid based metrics to occlusions/obstructions in the subject's environment. (Chapter 8)

\section{Organization of this Dissertation}

Chapter 2 describes research related to this project. First, I will present six of the fall risk assessments that are currently being used in practice and that the metrics developed here are compared against. Next, I will take a brief look at the weighted vest intervention used to evaluate these metrics. I will show prior work done in monitoring subject's ambulation in real time using external sensors, wearable devices, and direct measurement using various camera technologies, radar, and other means. I will show related work in gait computation using accelerometers, external sensors, and camera systems including the Microsoft Kinect. The chapter will conclude 
by exploring related work in clustering and outlier detection which is used as a starting point for the PCA based clustering and predictive analysis approaches presented here.

Chapter 3 contains the content of the paper entitled Metrics from In-Home Sensor Data to Assess the Effect of Clinical Therapies [10]. This full-length paper has been published by the Smart Health Journal. This paper presents all three groups of metrics and goes into detail describing how they were developed, verified using laboratory data, and validated against existing Fall Risk Assessments. The paper then discusses the use of the metrics to evaluate subjects undergoing the weighted vest therapy.

Chapter 4 presents an improved method of clustering walk data for residents with similar sets of features. Chapter 5 presents the Predictive Analysis technique which is developed as a way of predicting potential clinical events based upon changes in gait. Chapter 6 explores the long-term changes in metrics as a result of both clinical events as well as the strategic weighted vest therapy. Chapter 7 shows the correlation of these metrics to existing Fall Risk Analysis Instruments.

Chapter 8 measures the performance of the centroid based Stride Time, Stride Length, and Average Speed metrics against similar metrics developed by Erik Stone et al. [8], [12] using direct measurement of the feet. It also presents the results of a study using a simulated obstruction located in the center of the camera's field of view.

It should be noted that chapters 4 through 8 present different aspects of the project related to each of the project's contributions. Each chapter is presented with its own motivations, methods, results, and discussion sections.

Chapter 9 will provide an overall summary and discussion of this research. Lastly, chapter 10 will present the status of the work proposed during the comprehensive examination and presents some ideas about the next logical steps in the use of these metrics. 


\section{Chapter 2 Related Works}

In this chapter, we will examine current technologies and methods for assessing subject's ambulation both as standard of care in the healthcare fields and experimental methods. We will first examine an established set of existing fall risk instruments. While not a complete set, the set does examine both gait and balance and, when combined, provides a broad overview of the subject's condition. Next, I will briefly describe the weighted vest therapy. Following that, I will discuss currently used measurement modalities to assess gait. Next, I will explore other methods that have been used to measure gait, using both accelerometers, external sensors, and cameras. Lastly, I will discuss clustering and other techniques and algorithms that are used in this research.

\section{Fall Risk Assessments}

Fall risk assessments are divided into two broad categories, ones that characterize the subject's ability to balance and ones that characterize the subject's gait. Both are important as different subjects present differently, even for the same diseases. Genetics, medical history, upbringing, and physical environment will all play a part in a subject's individual condition.

\section{Balance}

$S P P B$

The Short Physical Performance Battery, developed by Guralnik et al. [13], consists of three groups of activities designed to gather an overview of the subject's condition as a predictor of the tendency to fall. The subject is asked to perform the following tasks:

1. Stand with feet together in a side-by-side position

2. Stand with feet together in a semi-tandem stance.

3. Stand with feet together in a tandem stance.

4. Walk forward 8 feet.

5. Rise from a chair and return to seated position 5 times.

The team found that the test can distinguish a gradient of risk for mortality and nursing home admission. Of 1122 subjects who were not disabled at the start of the study, those with the lowest 
scores on the performance test were 4-5 times more likely to have some disability at the 4 year follow up than those with the highest scores. Those whose score was in the intermediate range were 1.6-1.8 times more likely to have disability at the follow up than those with the highest scores [14]. Corsonello et al. found that SPPB qualified as an independent correlate of functional decline. An SPPB score $<5$ could identify patients experiencing functional decline during their follow-up. They found that SPPB was a valid instrument to identify patients at major risk of functional decline and death after discharge from an acute care hospital [15].

\section{Functional Reach}

A second common fall risk instrument is the Functional Reach Assessment. This test walks the patient through a series of reach related tasks designed to gauge not only the flexibility, but also the ability of the patient to balance sufficiently to reach. This instrument was published in 1990 by Duncan et al. [16] and focused on reach in the forward direction. The instrument measures the difference between the arm's length and the maximum forward reach using a fixed base of support.

Behrman et al. evaluated the effectiveness of the Functional Reach FRA and their findings indicate that the instrument using either one trial or three consecutive test trials effectively differentiated subjects with Parkinson's disease who have and who do not have a history of falling. They found that the FRA had high specificity though its sensitivity was rather low [17].

\section{Berg Balance Scale}

The Berg Balance Scale (BBS) is a test of 14 different items and is used to monitor fall risk principally relying on assessment of the patient's balance. The items examined are:
1) Sit to stand
2) Standing unsupported
3) Sitting unsupported
4) Stand to sit
5) Transfers 
6) Standing unsupported with eyes closed

7) Standing unsupported with feet together

8) Reaching forward with outstretched arm while standing.

9) Pick up object from floor from a standing position

10) Turn to look over left and right shoulder while standing

11) Turn 360 degrees

12) Place alternate foot on step stool while standing unsupported

13) Standing unsupported one foot in front

14) Standing on one leg

In this instrument, all 14 items are rated on a scale of 0 to 4 corresponding from no ability to full ability. The final score is simply the total of each rating out of a total of 56 with each item being equally weighted[18][19].

\section{Single Leg Stance}

The Single Leg Stance (SLS) assessment is done by asking the subject to stand unassisted on one leg and recording the time he/she was able to stand without touching the ground with the raised foot or touching the other leg [20]. This is repeated three times and the longest of the three attempts is used. Hurvitz et al. found out of 52 subjects, the 20 subjects who reported falling all had "significantly shorter" SLS time (9.6 seconds vs. 31 seconds) [20].

\section{Gait Related}

\section{Timed Up-and-Go}

The "Timed Up and Go" (TUG) test is a modification of the "Get Up and Go" test developed by Podsiadlo et al. in 1986 to add a temporal component to the assessment [21]. Their research has shown that the TUG test is reliable, correlates well with the Berg Balance Scale, Gait Speed, and Barthel Index of ADL, and lastly appears to predict the patient's ability to go outside alone safely [21]. Other researchers have arrived at similar conclusions [22], [23].

The test requires the patient to rise from an arm chair, walk 3 meters, turn around, return to the chair, and sit back down. The time required to perform this sequence of tasks is measured. 
Wall, et al. added timing of each individual component of the TUG [24] referred to as the "Expanded Timed Up and Go (ETUG)". By timing each specific component of the TUG, a more detailed assessment can be obtained. For example, the ETUG shows a significant difference between young and elderly subjects in walking speed with a less dramatic difference in the other parameters (for example, balance).

Reference values for the TUG have been published that are stratified based upon the patient's decade of age: 9.0 seconds for patients $60-69,10.2$ seconds for patients $70-79$, and 12.7 for patients 80-99 years old [25]. Morris et al. [26] investigated the reliability of this measure in people with Parkinson's disease. The TUG has been studied with other pathological conditions as well, including amyotrophic lateral sclerosis [27], post stroke [28], and orthopedic disturbances [29], [30].

\section{Habitual Gait Speed}

Habitual gait speed (HGS) has been studied as a means to detect a person's risk of falling [31][32][33]. To measure, the subject is asked to walk at their comfortable, habitual speed for a specified distance (20 feet is typical). The walk is timed, and the average speed is computed. Bohannon found gait speed to be highly reliable. This FRA was also shown to be moderately correlated with the subject's age $(-0.558 ; p<0.001)$, and minimally correlated with height and

several mechanical aspects of gait (ankle dorsiflexion strength, hip flexion strength, and hip abduction strength). Knee extension strength was found to be strongly correlated with HGS. Bohannon also published height normalized means and standard deviations for Habitual Gait Speed and Maximum Gait Speed [31].

\section{Strategically Weighted Vest Therapy}

Strategically weighted vests are currently being utilized to treat patients with multiple sclerosis, Parkinson's disease, and ataxia. One such BalanceWear ${ }^{\circledR}$ vest, produced by Motion Therapeutics 
in Oxnard, CA, consists of a vest, worn on the torso with Velcro areas where small, light weights are attached. The physical therapist performs the Balance-Based Torso-Weighting ${ }^{\mathrm{TM}}$ (BBTW) balance assessment on the patient to identify directional imbalance of a subject and strategically places $1 / 4$ to $1 / 2$ pound increments of weight in specific locations on the vest to improve balance and postural control. The patient is then instructed to wear the vest for several hours once or twice during the day. Studies conducted have focused on the clinical effects of the vest [34]-[36] but have not investigated how the vest specifically impacts the patient's ambulation beyond gait speed.

While there is some published research being conducted on the use of strategically weighted vests in other applications, there has been little to no research, outside of the studies referenced above, on the usefulness of these vests for improving the ambulation and reducing the risk of falls in the elderly. In [34], the author presents the case of a single patient who experienced improved balance and gait during static and dynamic activities. In [35] the authors expand upon the previous study to include 16 subjects with Multiple Sclerosis and found significant improvement in several clinical assessments of balance. A third study, [36], was a full clinical trial demonstrating immediate improvement of gait velocity and functional activity. In Gorgas et al., an increase in speed was seen in Multiple Sclerosis patients while no significant change was seen in cadence, step width or step length [37]. More recently, [9] has demonstrated the ability to measure changes in some metrics in the home environment. This paper more fully develops these and presents new metrics to serve as a basis to study the mechanisms by which the vest, and other, interventions improve a subject's ambulation. Gorgas, et al. in 2014, demonstrated significant increases in speed, cadence, and time spent in single limb support for both healthy control subjects as well as Multiple Sclerosis patients [38]. 


\section{Measurement Modalities}

\section{External Sensors}

Several techniques have been utilized to measure gait parameters in a laboratory or clinical setting. These will typically rely on markers worn by the subject that are detected by some form of sensor external to the patient or worn by the subject. The Vicon ${ }^{\circledR}$ Motion Capture System is an example. This system requires multiple infra-red reflecting markers to be worn by the subject, typically at the joints, to capture a skeletal model. Using several infra-red emitters and sensors, the position of each of the markers is captured and tracked in real time. Based upon the locations of specific sensors (for example, the ankles) gait parameters (stride time, step length, walking speed and others) can be computed automatically.

While this system is extremely accurate in monitoring the subject's position and works well in a laboratory or clinical environment, it is prohibitively expensive and invasive to use for home monitoring since it requires several infra-red cameras, emitters, and other equipment. In addition to the cost of procuring and installing the system, the subject would need to ensure that he or she is wearing the markers while they are performing their daily routines. For this reason, as well as the financial cost of installing the equipment, this method would likely be impractical.

\section{Subject Worn Sensors}

An alternative, and less expensive, solution is achieved by using sensors worn by the subject. This common approach [39]-[42] requires the subject to wear one or more sensors which convey the subject's motion to a processing computer. The sensors either transmit their data individually over a wireless network or they interface with a transmitter pack which, in turn, transmit the data from all of the subject's sensors. The transmitted information typically includes the subject's acceleration in each of three directions, and other related parameters. While this technology is less accurate than the Vicon ${ }^{\circledast}$ system, this type of system has been used to determine ambulation parameters since the cost to deploy is much lower[40][43]. 
The major drawback of this system, as with the previous system, is the necessity of wearing one or more sensors. For a subject to reliably wear the sensor, it must be designed to be put on, taken off, and activated easily. Not only must the subject remember to charge the device, they must also remember to wear the sensor when they are moving throughout their environment. If the subject were to wake in the middle of the night they may not remember or take the time to put on the sensor, leaving them unmonitored during a period when they are at a higher risk of falling. Lastly, there are psychological reasons why these wearable sensors may not be accepted by the elderly population which can range from simply being unwilling to wear the device, questioning the need for the device, or even associating the device with an admission of vulnerability [44].

\section{Direct Measurement}

What is needed, particularly for in-home monitoring, is an inexpensive system that does not require any direct interaction by the user. Several approaches have been used including multiple orthogonal webcams [45][46][47][48][49], using radar to monitor subject movement and or falls [50][51][52][53], acoustic [54][55][56], detecting interference with WiFi frequency signals [57], [58], [59], using a system of multiple laser range finders[60], [61], and passive infrared sensors [62], [63], [64]. An inexpensive depth camera, such as the Microsoft Kinect system, has been used to measure gait parameters by using the movement of the subject's feet to measure stride length, stride time and walking speed [65], [66]. While this system generates excellent results as compared to ground truth generated by a Vicon ${ }^{\circledast}$ Motion Capture System, it suffers when the subject's feet are occluded by objects in the subject's environment (tables, chairs, etc...).

\section{Measuring Gait}

Measuring gait is currently receiving a great deal of research attention with a wide range of goals including automating fall risk assessments [67]-[69], to broader characterization of a 
subject's gait. Originally, gait assessments were typically done in a clinical or laboratory setting, but with advances in computing power and the development of more capable camera systems, gait analysis is moving out of the clinical and into the home. This section will look at several different approaches to measure gait parameters. Four broad categories of sensors will be examined: accelerometers, other external sensors, cameras, and depth cameras.

\section{Accelerometers}

An accelerometer is a device, typically an integrated circuit or small device that can measure acceleration in one, two, or three directions. Using acceleration values over time, velocity and displacement can be calculated. While integrating acceleration and, then, velocity can be used to compute displacement, identifying specifically where the subject is located, and their velocity can still be problematic. While sufficiently detailed algorithms have been developed to compute gait parameters with acceptable accuracy, the precision of individual calculations can be low. As the stride parameters are ultimately based upon displacement, integrating from acceleration to location, particularly over a longer duration, the system can accumulate error.

Gonzalez et al. in [70] present an algorithm to find Initial Contact (IC) and Final Contact (FC) components in a step. They initially find IC's and FC's by identifying the zero crossing from positive to negative in the antero-posterior acceleration signal. Once this is found, they approximate the area of the positive values of this wavelet and, if it is above a specified threshold, they consider this an IC event.

Not all peaks, however, are candidates for inclusion. They must meet the following three criteria:

1) Vertical acceleration must be higher than gravity.

2) Peak must occur before acceleration reaches $99 \%$ of its local maximum value. 
3) If several peaks satisfy these conditions, the closest to the Zero Crossing is selected.

Once an IC is identified, the corresponding FC is selected as the first local minimum following the first detected local maximum.

In [71], Din et al. utilize a tri-axial accelerometer to compute gait parameters. They utilized four different algorithms to compute the following gait parameters: step time, stride time, swing time, and stance time. For the first two algorithms, their objective is to find IC and FC points utilizing an approach published in [72]. The vertical acceleration signal is smoothed by integrating

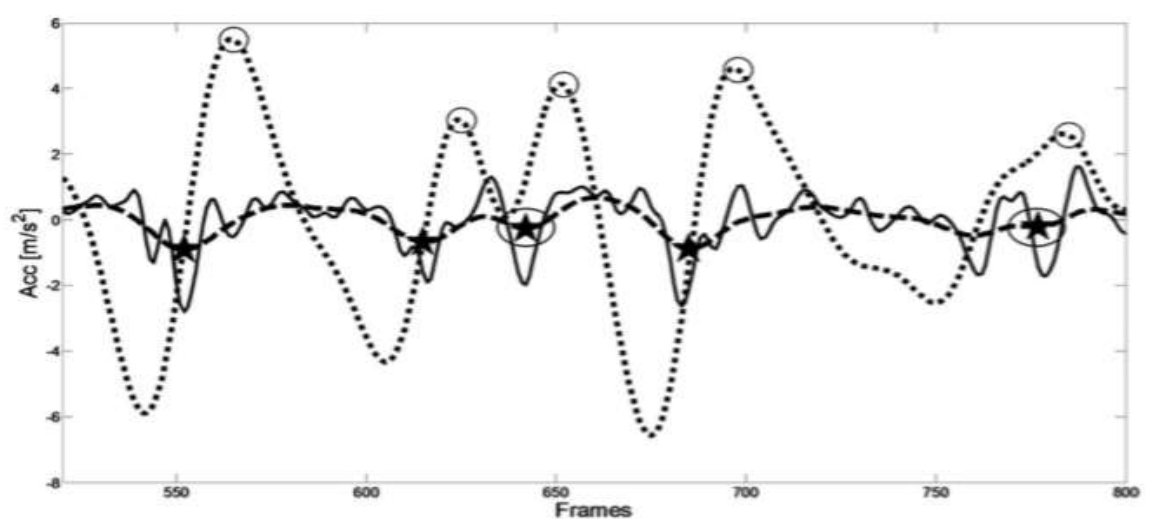

(a)

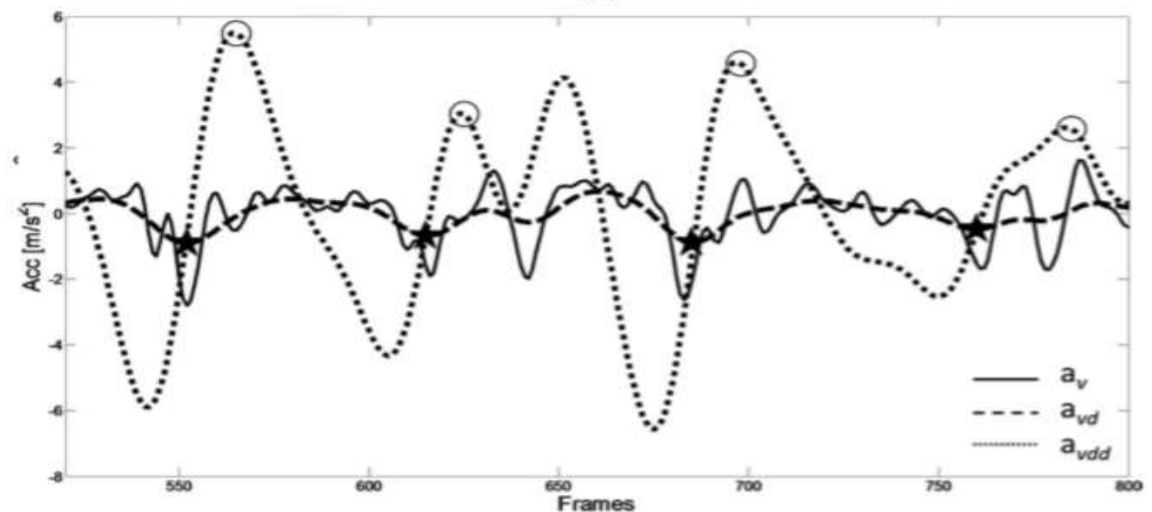

(b)

Fig. 4. Example of ICs (squares) and FCs (circles) detection during a single pass on the walkway. The black solid line represents $a_{v}$, the dashed line the differentiated with Gaussian CWT of $a_{v}\left(a_{v d}\right)$, and the dotted line the differentiated with Gaussian CWT of $a_{v d}\left(a_{v d d}\right)$. Panel (a) shows the IC/FC algorithm with spuriously detected IC events (circled squares). Panel (b) shows the correct detection of the ICs and FCs with the optimized technique.

Figure 2-1: Illustration of Algorithms 1 and 2. From [72]. 
then differentiating the signal using a Gaussian Continuous Wavelet Transform. IC events were identified as the times of the minima of the smoothed signal. Right and left IC's were designated by the sign of the filtered vertical axis angular velocity at the IC. The signal was differentiated once again, and the FC's are identified as the times of the maxima in new signal. Using a GaitRight mat as their ground truth, they achieved stride time measurement errors of $2 \%$ for IC's and $3 \%$ for FC's. The second algorithm is an optimization of the first that removes spurious IC events by applying a time window of 0.25 to 2.25 s following a previous IC. A diagram of these algorithms is shown in Figure 2-1. In the figure, the three traces show the vertical acceleration (solid line), the vertical acceleration differentiated with a Gaussian CWT (dashed line), and the vertical acceleration differentiated twice with the Gaussian CWT (dotted line). In the figure, the stars represent identified IC's and the circles represent identified FC's.

Their third algorithm uses an inverted pendulum model to estimate the distance traveled for each step. Utilizing the formula

$$
S L=2 \sqrt{2 l h-h^{2}}
$$

with $l$ equal to the distance between the accelerometer and the bottom of the foot and $h$ is the vertical distance traveled by the accelerometer computed using a double integration of the accelerometer's vertical data.

Their fourth algorithm computes Step Velocity using the Step Length computed by algorithm three and step time computed with algorithm two. Step Velocity is simply the Step Length / Step Time.

The study involved 60 subjects, 30 were diagnosed with Parkinson's Disease within the previous 4 months, and 30 age matched healthy subjects were used as a control. Table 2-1 shows the results of the validation of their gait measurements against data collected using the GaitRite 
Table 2-1: Validation results from accelerometer derived gait parameters as compared to the GaitRite system ground truth. From [72].

MeAN \pm SD Values and Mean Differences of 14 Gait Parameters Evaluated with an Instrumented WaLKWAy and BWM

\begin{tabular}{|c|c|c|c|c|c|c|c|c|}
\hline \multirow[t]{2}{*}{ Characteristic } & \multicolumn{4}{|c|}{$\mathrm{HC}$} & \multicolumn{4}{|c|}{ PD } \\
\hline & Instrumented walkway & BWM & Mean Difference, $\%$ & $p$ & Instrumented walkway & BWM & Mean Difference, \% & $P$ \\
\hline \multicolumn{9}{|c|}{ Mean gait characteristics } \\
\hline Step time (s) & $0.554 \pm 0.051$ & $0.567 \pm 0.054$ & $0.013,2.4 \%$ & $<0.001$ & $0.543 \pm 0.045$ & $0.555 \pm 0.047$ & $0.012,2.2 \%$ & $<0.001$ \\
\hline Stance time (s) & $0.715 \pm 0.080$ & $0.731 \pm 0.062$ & $0.016,2.2 \%$ & 0.018 & $0.706 \pm 0.072$ & $0.717 \pm 0.063$ & $0.011,1.6 \%$ & 0.119 \\
\hline Swing time (s) & $0.393 \pm 0.031$ & $0.404 \pm 0.051$ & $0.011,2.7 \%$ & 0.116 & $0.380 \pm 0.029$ & $0.394 \pm 0.044$ & $0.014,3.7 \%$ & 0.052 \\
\hline Step length (m) & $0.692 \pm 0.094$ & $0.718 \pm 0.099$ & $0.026,3.7 \%$ & 0.009 & $0.638 \pm 0.100$ & $0.681 \pm 0.089$ & $0.043,6.7 \%$ & $<0.001$ \\
\hline Step vel. $(\mathrm{m} / \mathrm{s})$ & $1.266 \pm 0.219$ & $1.275 \pm 0.220$ & $0.009,0.7 \%$ & 0.606 & $1.187 \pm 0.223$ & $1.242 \pm 0.218$ & $0.055,4.6 \%$ & 0.007 \\
\hline \multicolumn{9}{|c|}{ Variability (var) gait characteristics } \\
\hline Step time var (s) & $0.017 \pm 0.008$ & $0.024 \pm 0.011$ & $0.007,32.8 \%$ & 0.002 & $0.022 \pm 0.012$ & $0.031 \pm 0.023$ & $0.009,41.8 \%$ & 0.009 \\
\hline Stance time var $(s)$ & $0.022 \pm 0.012$ & $0.026 \pm 0.015$ & $0.005,21.8 \%$ & 0.039 & $0.023 \pm 0.010$ & $0.033 \pm 0.022$ & $0.011,46.3 \%$ & 0.003 \\
\hline Swing time var (s) & $0.016 \pm 0.009$ & $0.027 \pm 0.024$ & $0.011,66.3 \%$ & 0.015 & $0.019 \pm 0.007$ & $0.031 \pm 0.025$ & $0.013,65.9 \%$ & 0.004 \\
\hline Step length var (m) & $0.023 \pm 0.007$ & $0.043 \pm 0.021$ & $0.021,92.6 \%$ & $<0.001$ & $0.025 \pm 0.008$ & $0.040 \pm 0.015$ & $0.015,57.6 \%$ & $<0.001$ \\
\hline Step vel. var (m/s) & $0.058 \pm 0.017$ & $0.043 \pm 0.021$ & $-0.015 .25 .4 \%$ & 0.008 & $0.062 \pm 0.023$ & $0.040 \pm 0.015$ & $-0,022,35.8 \%$ & $<0.001$ \\
\hline \multicolumn{9}{|c|}{ Asymmetry (asy) gait characteristics } \\
\hline Step-time asy (s) & $0.010 \pm 0.006$ & $0.015 \pm 0.014$ & $0.005,38.2 \%$ & 0.151 & $0.023 \pm 0.027$ & $0.023 \pm 0.044$ & $-0.0001,0.3 \%$ & 0.990 \\
\hline Stance time asy (s) & $0.020 \pm 0.012$ & $0.014 \pm 0.013$ & $-0.006,30.9 \%$ & 0.035 & $0.022 \pm 0.009$ & $0.023 \pm 0.045$ & $0.001,6.9 \%$ & 0.846 \\
\hline Swing time asy (s) & $0.010 \pm 0.010$ & $0.013 \pm 0.015$ & $0.003,29.7 \%$ & 0.387 & $0.018 \pm 0.016$ & $0.019 \pm 0.024$ & $0.001,4.8 \%$ & 0.848 \\
\hline Step length asy (m) & $0.022 \pm 0.016$ & $0.014 \pm 0.017$ & $-0.008,37.8 \%$ & 0,049 & $0.024 \pm 0.017$ & $0.014 \pm 0.015$ & $-0.010,4.6 \%$ & 0.023 \\
\hline
\end{tabular}

Mean difference: BWM - Instrumented walkway; Step vel.: Step velocity.

Mat as ground truth. Focusing on the mean and variability, Step Time and Step Length had errors between mean of $2.4 \%$ and $3.7 \%$ respectively. These measurements were, however, quite variable as compared to the GaitRite system. Accelerometer computed step time showed a $32 \%$ increase in variability while step length showed a $92 \%$ increase in variability.

In a more recent study, van Schooten et al. looked at using accelerometer data to predict the likelihood that subjects will experience a first and a second fall [73]. They assessed habitual physical activity and gait quality utilizing a collection of metrics. Amount of physical activity was measured using duration of locomotion, time sitting, standing, and lying, the number of strides, duration of walks, and number of transitions to stance among others. Gait quality was measured with metrics including stride frequency, walking speed, stride length, stride frequency, gait 
symmetry, gait smoothness, sample entropy, and gait intensity (Root-mean-square (RMS) of the signal).

With a total of 39 different metrics (6 general metrics +11 metrics $\times 3$ directions), van Schooten et al. utilized principle component analysis to dimensionally reduce the number of features considered. They selected components with an eigenvalue $>1$. The 18 principle components selected explained $80.5 \%$ of the variance.

A total of 319 older people were included in this study with $51 \%$ female and $90 \%$ community dwelling and the average age was 75 years old. Results showed that fall history, alcohol consumption, and gait quality predicted time to first fall with adequate to good accuracy. Adding in a factor related to strength, time to second fall is predicted with good accuracy. The study also shows high degree of correlation between various quality of gait parameters. For example, walking speed was highly correlated with the RMS of the vertical signal. Walking speed was also correlated with the magnitude of the dominant frequency.

Carbonaro et al. developed the FootMoov smart shoe device for monitoring gait [74]. This device has a built-in tri-axial accelerometer and force sensors. Combined with a gait phase detection algorithm, using both force and inertia information, they are able to reliably detect gait phases to within $45 \mathrm{~ms}$. The device transmits data wirelessly to a nearby smartphone for processing and storage.

Bamberg et al. developed a more advanced and full featured device called the "GaitShoe" system consisting of a pair of heavily instrumented shoes designed to provide a comprehensive measurement of gait [40]. A diagram of the shoe is shown in figure 2-2. The shoe contains accelerometers to measure stride length, stride velocity, and other velocities and displacements. Gyroscopes are used to measure the orientation of the shoe. Force sensitive resistors measure 
the pressure across the foot and are principally used to capture HS and TO events. Bend sensors are used to sense the degree to which the foot bends during steps, and electric field sensors are used to measure the shoe's height above ground.

Initial results show promise in the ability to measure gait in both healthy subjects ( 3 male, 7 female) and subjects with Parkinson's Disease ( 2 male, 3 female). Results evaluate favorably against data collected using the motion capture facility at Massachusetts General Hospital Biomotion Laboratory.

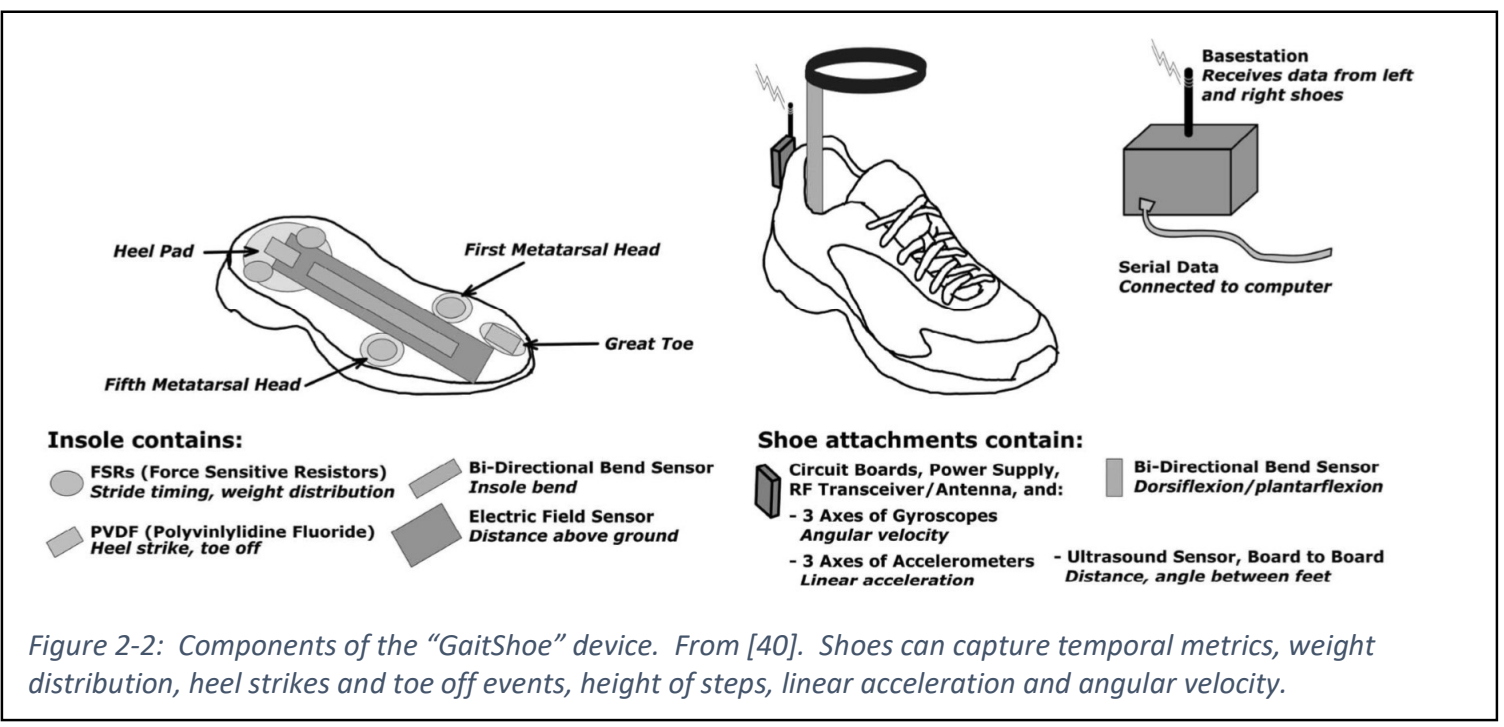

Gait Measurements Using External Sensors

A better method would be one that utilizes a direct measurement of step length and time.

The GaitRite system is one commercial example of this approach. Used as ground truth for the accelerometer studies mentioned above, this system boasts a high degree of accuracy. McDonough et al. validated the GaitRite system versus pencil/paper and video based methods [75]. In the study, one 27 year old healthy female subject was asked to walk across the GaitRite mat at various rates and degrees of step symmetry. The monitored metrics included speed, right and left step times, and right and left step lengths. Pencil/paper measurements were taken by placing a strip of paper, similarly sized to the mat, over the GaitRite mat. The soles of the subject's 
shoes were colored using blue carpenter's chalk such that as the subject walked on the paper, the steps were shown on the paper. A camera system was also used to video the subject's walks to provide a second ground truth with which to compare.

Table 2-2 shows the results of their study. There is generally good agreement between the GaitRite values and the other methods with one the distance step length agreeing more closely with the paper/pencil method and step time agreeing better with the video methods. For walking speed, the GaitRite system generally returned values between the pencil/paper method (usually lower except in "Step-to"), and video (higher).

Table 2-2: Results from McDonough et al. comparing paper-pencil and video methods of measuring gait parameters vs. the GaitRite system. From [75].

Table 1: Results of Gait Analysis Performed Concurrently by 3 Methods: Paper-and-Pencil (PP), Video (VB), and GAITRite (GR) System

\begin{tabular}{|c|c|c|c|c|c|c|c|c|c|c|c|c|c|c|c|c|c|c|c|}
\hline \multirow{3}{*}{\multicolumn{2}{|c|}{ Trial Description }} & \multicolumn{3}{|c|}{$\begin{array}{l}\text { Walking Speed } \\
(\mathrm{cm} / \mathrm{s})\end{array}$} & \multicolumn{3}{|c|}{$\begin{array}{c}\text { Cadence } \\
\text { (steps/min) }\end{array}$} & \multicolumn{6}{|c|}{ Mean Step Length $(\mathrm{cm})$} & \multicolumn{6}{|c|}{ Mean Step Time (s) } \\
\hline & & \multirow[b]{2}{*}{$\mathrm{PP}$} & \multirow[b]{2}{*}{ VB } & \multirow[b]{2}{*}{$\mathrm{GR}$} & \multirow[b]{2}{*}{$\mathrm{PP}$} & \multirow[b]{2}{*}{ VB } & \multirow[b]{2}{*}{ GR } & \multicolumn{3}{|c|}{ Right } & \multicolumn{3}{|c|}{ Left } & \multicolumn{3}{|c|}{ Right } & \multicolumn{3}{|c|}{ Left } \\
\hline & & & & & & & & PP & VB & GR & PP & VB & GR & PP & VB & GR & PP & VB & GR \\
\hline 1 & Fast & 154 & 190 & 165 & 116 & 111 & 113 & 85.00 & 119.12 & 85.84 & 88.43 & 117.54 & 89.33 & 0.55 & 0.45 & 0.48 & 0.57 & 0.53 & 0.56 \\
\hline 4 & Slow & 82 & 101 & 89 & 86 & 82 & 85 & 60.93 & 49.87 & 60.57 & 62.50 & 62.52 & 64.21 & 0.74 & 0.69 & 0.70 & 0.76 & 0.73 & 0.70 \\
\hline 5 & Wide base & 57 & 72 & 68 & 68 & 67 & 77 & 60.32 & 77.67 & 54.46 & 59.37 & 64.80 & 53.09 & 1.06 & 0.81 & 0.76 & 1.04 & 0.81 & 0.80 \\
\hline 6 & Narrow base & 48 & 64 & 57 & 72 & 75 & 77 & 40.41 & 58.61 & 40.36 & 48.54 & 67.47 & 48.26 & 0.84 & 0.78 & 0.76 & 1.01 & 0.84 & 0.80 \\
\hline 7 & Toe-in & 53 & 68 & 58 & 80 & 79 & 77 & 43.95 & 70.05 & 43.95 & 52.29 & 75.10 & 48.13 & 0.83 & 0.72 & 0.78 & 0.98 & 0.82 & 0.79 \\
\hline
\end{tabular}

The authors conclude that the GaitRite system is "a valid and reliable tool" as well as point out the advantages of portability, (relatively) low cost, and simplicity in connecting to a PC compatible computer through a serial port.

The GaitRite system is constructed with two columns of 256 sensors along a mat measuring approximately 3 meters [75]. Four years later, Middleton et al. published a design for a similar mat but with a denser sensor array to capture more detail about foot placement and location. While they also use a mat length of $3 \mathrm{~m}$, the sensor density was increased by a factor of 
6 (16 columns of 96 sensors each). This system can be constructed with components totaling less than $\$ 100$ (2005 dollars) and is comparable in performance to the GaitRite system.

While more accurate than accelerometers, these systems require the subject to only walk along the mat, or to create a surface the size of the subject's room. The first is not feasible in the home setting while the second could be prohibitively expensive.

\section{Gait Measurements Using Cameras}

To monitor a subject's gait outside of a laboratory or clinic setting which is, after all, the goal of this research, it is ideal to use a data collection system that does not rely on hardware which is worn by the subject. Since monitoring is to be performed in a home setting, reducing the cost of the system is also an important goal. One way of meeting both of these constraints is by using cameras. This section will take a look at different methods of qualitatively and/or quantitatively measuring gait using one or more 2-D camera systems.

The first study was done by Miaou et al. [76] and uses a spherical camera system to generate an image of the entire room. Background subtracting using an average of ten background images is done to isolate the subject. A very simple fall detection algorithm is then used to classify the subject as standing or as having fallen. The vertical and horizontal lengths of the subject's image is calculated and the ratio of height to width is computed. In the event of a fall, this value will change significantly, and it is this change that is used to trigger a fall event. Specifically,

Table 2-3: Results from Miaou et al. [76]. Table II shows a truth table for fall recognition vs. actual falls. The left half of the table shows the rates with no optimization using personal information (specifically BMI). The right half of the table uses that information to improve results.

TABLE II. RESULTS OF RECOGNITIONS WITH AND WITHOUT PERSONAL INFORMATION

\begin{tabular}{c|c|c|c|c}
\hline & \multicolumn{2}{|c|}{$\begin{array}{c}\text { Without personal } \\
\text { information }\end{array}$} & \multicolumn{2}{|c}{$\begin{array}{c}\text { With personal } \\
\text { information }\end{array}$} \\
\hline $\begin{array}{c}\text { System Fall accident } \\
\text { recognition }\end{array}$ & Yes & No & Yes & No \\
\hline Positive & 25 & 10 & 30 & 8 \\
\hline Negative & 8 & 17 & 3 & 19 \\
\hline \multicolumn{4}{c}{ TABLE III. }
\end{tabular}
FINAL SYSTEM PERFORMANCE

\begin{tabular}{c|c|c}
\hline & $\begin{array}{c}\text { Without personal } \\
\text { information }\end{array}$ & $\begin{array}{c}\text { With personal } \\
\text { information }\end{array}$ \\
\hline Accuracy & 0.70 & 0.81 \\
\hline Sensitivity & 0.78 & 0.90 \\
\hline Specificity & 0.60 & 0.86 \\
\hline Kappa & 0.68 & 0.80 \\
\hline
\end{tabular}


out of six successive frames, if the first three ratios are greater than one, and the last three ratios are less than one and descending, then a fall is possible. Lastly, the last two ratio changes must be greater than a threshold. To compensate for different ratios due to different body sizes, the subject's body mass index is used to adjust the ratios.

Using a group of 20 people of varying heights $(151-180 \mathrm{~cm})$ and weights $(<50 \mathrm{~kg}$ to $>80 \mathrm{~kg})$, they obtained the results shown in table 2-3. The accuracy of their technique was found to be $70 \%$ without using personal information and rising to $81 \%$ when personal information is included. Sensitivity is $78 \%$ and $90 \%$ respectively and specificity is $60 \%$ and $86 \%$. This suggests that even with BMI used to improve results, nearly one in five events are misclassified.

Time of Flight (ToF) cameras use the reflection of light to estimate distance from the camera. The two dimensional "image" contains distances to the nearest object instead of light intensity or colors as one might expect using a standard camera. Altuntas et al. conducted a study using the ToF camera to study human motion [77] in one male subject. The camera used in the study had a measurement accuracy of $2 \mathrm{~cm}$ at the maximum distance $(5 \mathrm{~m})$. For higher precision, the authors recommend temporal filtering as the range data can be quite noisy. They opted not to filter the data for this study, however, as the precision was not necessary to estimate joint angles. The camera was placed $1 \mathrm{~m}$ from the subject, who was

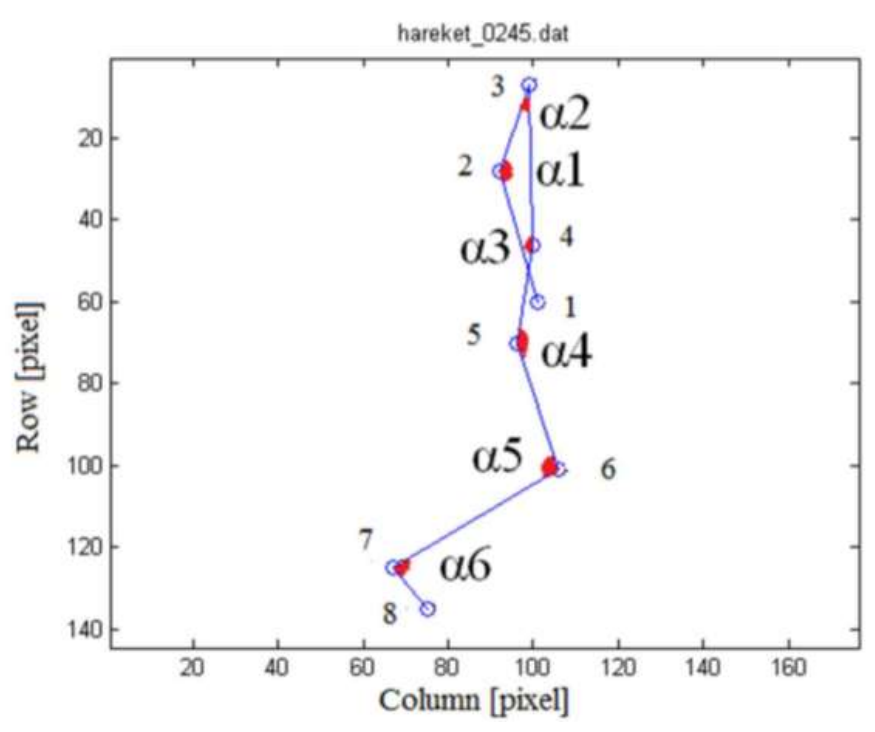

Figure 2-3: Location of joints (red dots) of the subject taken using a ToF camera. Taken from [77]. 
running on a treadmill. The 3-D coordinates were computed using the intensity values combined with the measurement data for each frame. Figure 2-3 shows the location of the joints, as extracted from the camera images, of the runner. Figure 2-4 shows the variations of the angles

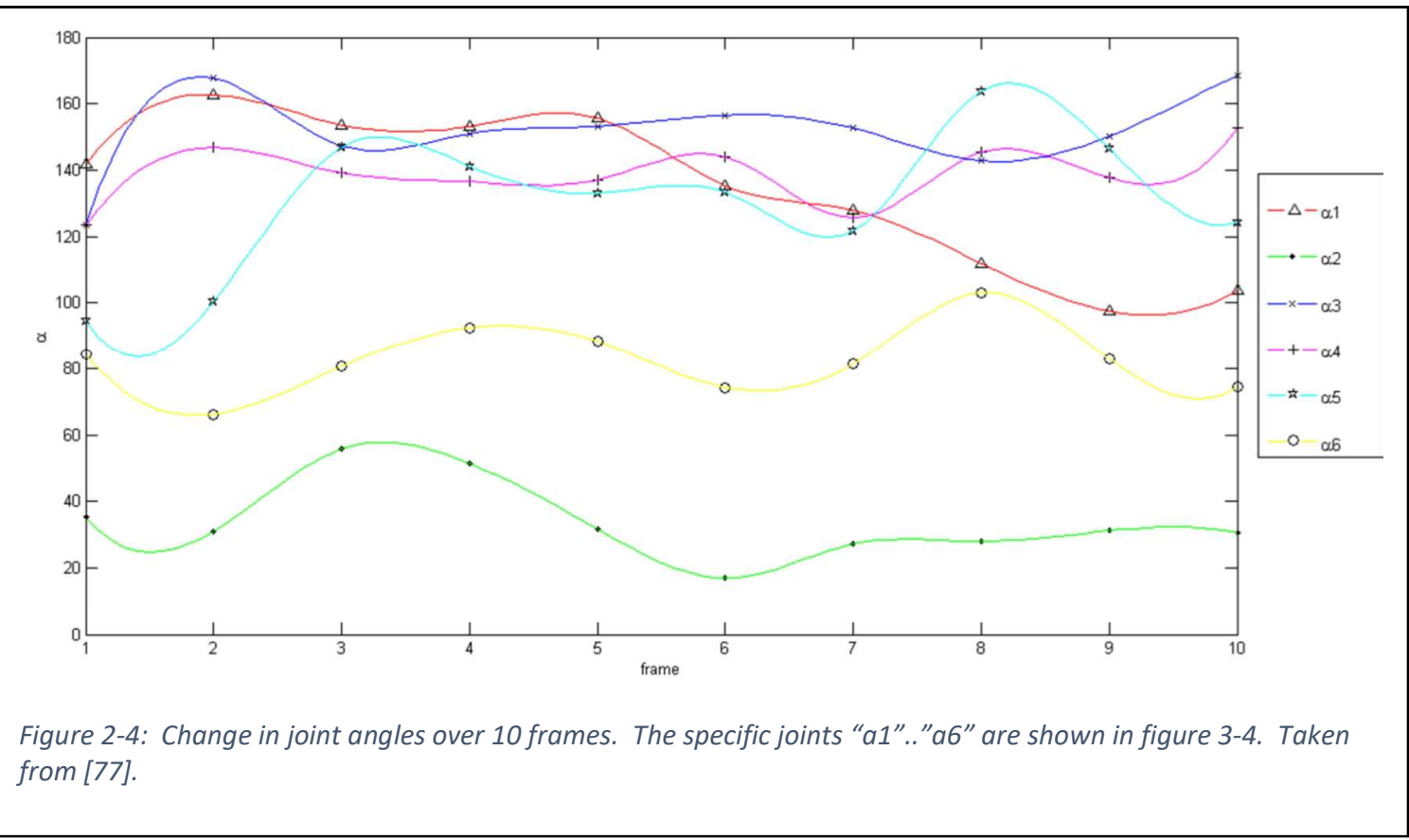

between the joints over 10 frames. As can be seen from these figures, at least visually, the ToF camera system is able to isolate and track the subject's motion.

\section{Microsoft Kinect Depth Camera System}

The Microsoft Kinect depth camera system is marketed by Microsoft as an accessory to their X-Box gaming platform. In addition to a standard 2-D visual light camera, it also has an infrared depth camera which can measure distance to the subject. The second-generation Kinect device depth camera component works similarly to the ToF camera system described in the previous section but is more accurate with less noise. Recently, this camera has received quite a bit of attention from research groups due to its low cost, ease of use, and accuracy.

Ejupi et al. utilize a Kinect depth camera system to directly assess fall risk using the choice reaching and stepping reaction time fall risk assessment[78]. This FRA, developed by Lord and 
Fitzpatrick [79], evaluates a person's risk of falling by having them step on or reach for one of four randomly illuminated panels. Ejupi et al. looked at 94 community dwelling older people including 20 who participated in the study at home. Using the skeletal data, reaction time, total time, and movement features were computed. They determined that the choice reaching reaction time, as measured using the Kinect, was effective at differentiating between fallers and non-fallers. The choice stepping reaction time, however, was not effective at classifying the two groups of people.

Chun et al. utilized a pair of Kinect cameras to monitor balance[80]. Subjects were asked to maintain their balance on a movable base plane while their movement was tracked using the Kinect skeletal data. These results were compared to ground truth data collected by the Vicon motion capture system. Their results show accurate results with high correlation between the hip and knee flexion - extension angles. Poorer results were seen when lower limb angles were considered. The authors conclude that clinically acceptable accuracy can be achieved by the Kinect when monitoring subject's movements to maintain balance.

Another study was undertaken by Cippitelli et al. [81]. This study presents an algorithm to locate and estimate the trajectories of up to six joints extracted from the side depth view of the Kinect. The group then uses this information to compute a "Get Up and Go Test" score based upon the gait information extracted from the joint motion.

The Kinect is mounted at a height of $92 \mathrm{~cm}$ from the floor and a distance of $330 \mathrm{~cm}$ from the wall. These values reflect a trade-off between pixel density and sensor coverage area and requires the height of the subject to be limited. The floor is carpeted to reduce reflections from the floor. Initially, 100 frames are collected and averaged to create a background image. Any point where the difference between the foreground and background depth value is less than a 
threshold is set to 0 . If the difference is greater than the threshold, then the resulting value is set to 1 . The end result of this is a silhouette of the subject.

The joint positions in the silhouette are then estimated using anthropometric models along with the assumption that the distance between joints do not change. For example, the head is located and used as a reference point. The shoulders are located a fixed distance from the top of the head along the vertical axis. This estimate is then improved by estimating the headshoulder angle. The elbow joint angle, as another example, is estimated by examining the pixels around the elbow. Starting from the shoulder joint, a vector is rotated through 180 degrees. The number of overlapping pixels are computed and the joint angle is determined to be the angle that maximizes the number of pixels matched.

This algorithm was validated against the Vicon ${ }^{\circledR}$ motion capture system. The results of the validation are shown in Table 2-4. "Algorithm 1" is the skeletal modeling algorithms in the Kinect system itself and "Algorithm 2" is the OpenNI Software Development Kit. As can be seen from the table, the joint angle measurements are significantly improved over the second and third algorithms. While some results deviate by close to $50 \%$, other joint angles are quite accurate (ankle angle, in particular).

A second, more recent, study by Muller et al. [82] created a motion study laboratory with comparable accuracy to the Vicon ${ }^{\circledR}$ system. The system was created using a total of six Kinect systems
Table 2-4: Comparison of joint angles computed by the proposed algorithm, and two other algorithms, to Vicon ${ }^{\circledR}$ motion capture results. From [81].

\begin{tabular}{ccccccc}
\hline Joints & \multicolumn{2}{c}{$\begin{array}{c}\text { Proposed } \\
\text { vs. } \\
\text { Marker }\end{array}$} & \multicolumn{2}{c}{$\begin{array}{c}\text { Algorithm 1 } \\
\text { vs. } \\
\text { Marker }\end{array}$} & \multicolumn{2}{c}{$\begin{array}{c}\text { Algorithm 2 } \\
\text { vs. } \\
\text { Marker }\end{array}$} \\
\hline & $\mu$ & $\sigma$ & $\mu$ & $\sigma$ & $\mu$ & $\sigma$ \\
head & $\mathbf{2 . 3 3 3 9}$ & $\mathbf{1 . 1 4 2 6}$ & 5.8878 & 3.0238 & 5.5202 & 2.8346 \\
shoulder & $\mathbf{4 . 4 1 5 4}$ & $\mathbf{2 . 1 3 9 2}$ & 5.3514 & 2.4382 & 7.4386 & 4.8854 \\
elbow & $\mathbf{4 . 0 1 6 6}$ & $\mathbf{1 . 9 9 1 7}$ & 5.4609 & 2.0548 & 8.1691 & 4.4949 \\
ankle & $\mathbf{3 . 0 3 8 6}$ & $\mathbf{2 . 9 9 6 1}$ & 5.2701 & 3.8576 & 12.2962 & 13.1106 \\
knee & $\mathbf{3 . 5 1 3 2}$ & $\mathbf{1 . 7 9 9 7}$ & 7.9368 & 5.706 & 9.9547 & 8.1032 \\
hip & $\mathbf{7 . 2 5 0 8}$ & $\mathbf{3 . 7 0 0 7}$ & 11.0742 & 6.3248 & 10.0549 & 5.2083 \\
\hline
\end{tabular}


arranged "as an avenue" in two rows of three surrounding a motion capture area. The volume of this area was chosen to match a typical Vicon system volume. Each row of cameras was placed roughly $2.5 \mathrm{~m}$ apart and the rows were separated by $2 \mathrm{~m}$. The cameras were placed looking along the rows and turned inward 35 degrees. The subject would then walk along the avenue towards the cameras. Taken as a whole, the Kinect camera systems created an area $9 m$ by $2 m$ of complete monitoring by at least two cameras.

Each Kinect was attached to a dedicated small form-factor computer which, in turn, was connected to a network switch along with a server machine which ultimately received and processed the data. All six data collection machines as well as the server computer were time synchronized to within milliseconds using the Precision Time Protocol. Each of the six clients transmit data to the server on demand by the server computer.

A 3-D scene is formed by combining the point clouds generated by each of the clients after transforming their coordinate systems to a room based coordinate system. This is possible because the set of cameras are calibrated to a global coordinate system enabling a single representative point cloud located at a specific location in the motion capture area.

The system was validated against the Vicon ${ }^{\circledR}$ motion capture system with 10 healthy subjects ranging in age from 18 to 35 years old. The Vicon system was configured with 10 cameras and is accurate to $1 \mathrm{~mm}$. Table 2-5: Results comparing the Kinect based motion capture system to the Vicon motion capture system. Results show nearly identical results across all measured metrics. From Muller et al. [81]

Twelve markers were affixed to each subject and data were recorded at 120 frames per second. The results of their validation are shown in

\begin{tabular}{l|l|l}
\hline Parameter & Our System & VICON \\
\hline AV Walking Speed $(\mathbf{c m} / \mathbf{s})$ & Mean \pm SD & Mean \pm SD \\
\hline Step time L (s) & $\mathbf{1 2 3 . 0 6} \pm \mathbf{1 4 . 2 6}$ & $\mathbf{1 2 2 . 5 3} \pm \mathbf{1 4 . 5 0}$ \\
\hline Step time R (s) & $0.60 \pm 0.07$ & $0.61 \pm 0.07$ \\
\hline AV step time L (s) & $0.60 \pm 0.07$ & $0.60 \pm 0.07$ \\
\hline AV step time R (s) & $0.60 \pm 0.07$ & $0.61 \pm 0.07$ \\
\hline AV step time AVG (s) & $0.60 \pm 0.06$ & $0.60 \pm 0.06$ \\
\hline
\end{tabular}


Table 2-5. Looking at the results, it is easy to see similar performance between both systems. When there is a discrepancy between a pair of metric values, the difference is only a small fraction of the measured standard deviation.

The results of this study show that the Kinect camera, particularly when combined, can have comparable performance to the "gold standard" Vicon system. The authors did notice that the skeletal fitting algorithm in the Kinect depends heavily on the viewing angle from which the sensor tracks the subject's movement. If the subject is viewed from the side, the Kinect will attempt to interpolate the position of an occluded leg passing behind the leg closer to the camera. As a gaming device, this is acceptable, but as a gait research tool, this is not desirable. For this reason, the authors utilized pairs of cameras viewing the subject from opposite sides.

Nguyen et al. developed an abnormal gait detection system using the skeletal data from a Kinect[83]. The skeletal data from each frame is decomposed into gait cycles and represented by a feature vector describing the relationship between pairs of bones and joints. These feature vectors are clustered and codewords are assigned to each cluster. These codewords are then used to model "normal" human gait. Deviations from this "normal" model are considered abnormal. They report an accuracy of $90 \%$ in distinguishing normal from abnormal gait.

Pfister et al. also compared the performance of the Kinect to the Vicon[84]. 20 healthy adults were monitored walking and jogging at three different velocities on a treadmill. While the Kinect did capture the overall nature of the subject's gait, it fell short with specific measurements of hip and knee flexion and extension. Hip angular displacement turned out to be highly inaccurate. Stride time measurements, however, showed better results, particularly at slower speeds. The authors conclude that while it can, with some "minor adjustments", accurately 
measure gait, it will still require significant advances in hardware and software before it can be used in a clinical setting.

Galna, et al. also compared the performance of the Kinect platform to the Vicon system [85]. In this study, fine motor control was also looked at along with gross motor control including gait. They concluded that the Kinect consistently performs well with timing repetitive movement. Additional, good performance is seen with gross movements (sit to stand as an example) but poor performance is seen with fine movements (such as hand clasping).

Xu et al. studied the accuracy of the Kinect in measuring gait parameters on a treadmill. The study tracked heel strike (HS) and toe off (TO) events, using the data to compute step time, stride time, stance time, swing time, and double support time metrics [86]. The study also explored the use of the skeletal models to follow joint trajectories of the knee and hip joints. Their results show significant magnitude error when following joint trajectories. Based upon other published criteria, they determined that the Kinect is unable to measure gait parameters using the skeletal model. Better results were also seen when computing metrics using the HS events as opposed to the TO events. They speculate that HS data produced better results simply due to the positioning of the sensor. The sensor was placed at the front of the treadmill and, consequently, was closer to the HS than the TO events resulting in more accurate measurement of the HS events.

While many studies have shown that tracking gait using the skeletal data provided by the Kinect can be problematic, Gabel et al. [87] present a methodology that utilizes the Kinect to produce a full body gait measurement system. By using regression trees on the entire skeletal model, the authors are able to refine the skeletal data to produce accurate readings of gait parameters. Stride time measurements, for example, are accurate to within $1 \%$ and are reported to improve upon the results in [8]. 
Another gait analysis system using the skeletal model that achieved good results was published by Clark et al. [88]. As part of their study, many metrics were derived, though it was found that many were redundant, as determined through correlation. The subset used include step length, step length asymmetry, foot swing velocities, and the difference between peak and mean velocity. The group found that all the Kinect derived metrics proved to be reliable measurements (Intraclass correlation coefficients $>0.8$ for all metrics). While the study was done using subjects who had had strokes, the authors make no assertion as to the reliability of using this system for subjects who have recently had a stroke, leaving that for future work.

Dolatabadi et al. presented a direct comparison between the Kinect and the GaitRite system [89] in measuring gait. Twenty healthy adults performed several sequences of walks including fast and normal pace. Step Length, Step Time, and speed were among the metrics evaluated. Looking at Bland-Altman limits, Intraclass correlation coefficients, and inter-trial reliability results, they conclude that the Kinect is capable of measuring gait parameters in healthy adults.

At the Center for Eldercare and Rehabilitation Technology at the University of Missouri, the Kinect has been used to measure and monitor gait since 2011. The first studies performed with the Kinect [66] focused on measuring gait by monitoring the movement of the legs and, unlike the majority of studies at the time, opted not to use the skeletal model data due to instability and noise. Another key difference with this study is it was performed in a home setting and not in a laboratory or clinic.

The depth data are first read from the Kinect and the background subtracted using a simple background subtraction algorithm. All values less than a depth threshold are considered foreground while the remainder are considered background and removed. The remaining depth 
information is filtered using a block based filtering algorithm to remove noise and smoothed. The resulting collection of data is a point cloud representation of the subject.

The centroid of the point cloud is computed next and projected onto the ground plane. To compute walking speed, this centroid value is first filtered with a moving average filter to reduce noise. After, it is a simple matter to compute the distance traveled by the centroid over a specific length of time to generate walking speed.

Table 2-6: Differences in Walking Speed, Stride Time, and Stride Length as compared to the Vicon results. From [66].

$\%$ DIFFERENCE IN WALKING SPEED COMPARED TO VICON

\begin{tabular}{l|c|c|c}
\hline & Kinect \#1 & Kinect \#2 & Web-Camera \\
\hline Mean \% Diff & -4.1 & -1.9 & 4.4 \\
\hline Std. Deviation. & 1.9 & 1.2 & 4.8 \\
\hline
\end{tabular}

To compute the temporal gait parameters, only the points less than 20 inches from the ground plane are used. These points are projected to the ground plane, normalized by subtracting the mean, and rotated

\% DifFERENCE In STRIDE TIME COMPARED TO ViCON

\begin{tabular}{l|c|c|c}
\hline & Kinect \#1 & Kinect \#2 & Web-Camera \\
\hline Mean \% Diff & 1.9 & 0.7 & -2.3 \\
\hline Std. Deviation. & 2.5 & 1.3 & 2.1 \\
\hline
\end{tabular}

\% DifFERENCE IN STRIDE LENGTH COMPARED TO ViCON

\begin{tabular}{l|c|c|c}
\hline & Kinect \#1 & Kinect \#2 & Web-Camera \\
\hline Mean \% Diff & -1.9 & -1.1 & 0.2 \\
\hline Std. Deviation. & 3.9 & 2.5 & 1.6 \\
\hline
\end{tabular}
based upon the localized walking direction. The number of right and left steps are derived from the time series of the correlation coefficient. The signal is filtered to remove noise and to smooth, and the footsteps are extracted with right steps being detected as local minima, and left steps are local maxima. Once these points are determined, the calculation of stride time and stride length are straight forward.

Stride time, stride length, and walking speed were compared against Vicon ${ }^{\circledR}$ measurements and the results are shown in Table 2-6. Walking speed and stride time showed better results than using multiple orthogonal webcams while the stride length calculation was 
better using the webcam. Clearly, all three metrics compared favorably to the Vicon ${ }^{\circledR}$ generated metrics.

A subsequent study, also by Stone et al. [8] more fully develops the use of the Kinect to measure gait parameters. In this study, the overall approach was the same with some optimizations added to improve quality. First, a minimum height criterion was added to filter point clouds which did not sufficiently capture the resident. Secondly, a state machine was developed to cull activity that is not considered to be a purposeful walk. A diagram of the state

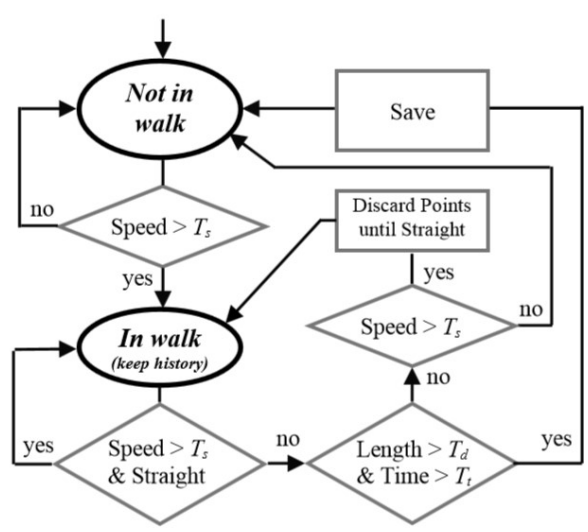

Figure 2: State machine diagram of the walk identification algorithm. The values $T_{s}, T_{d}$, and $T_{t}$ are thresholds for speed, distance, and time, respectively. These thresholds, along with a straightness criterion, are used to determine the minimum characteristics of walks that are saved. For this work, threshold values were: $T_{s}=5 \mathrm{in} / \mathrm{sec}, T_{d}=48 \mathrm{in}, T_{t}=1.0 \mathrm{sec}$.

Fiqure 2-5: From Stone et al. $[81$

machine is shown in figure 2-6. To be considered a purposeful walk, the motion must be at a speed greater than $5 \mathrm{in} / \mathrm{s}$, must be longer than 48 inches in length, and must exceed 1 second in duration. Additionally, the walk must be reasonably straight as curved walks can negatively impact the extracted gait parameters. Historical data are also used to facilitate tracking subjects. Lastly, potential walks are discarded if they are occluded, show problems with segmentation, or contain fewer than 6 steps. 
Results show the

ability to track walking speed,

stride time, and stride length in

real time (15 frames / s) over

several months. Figure 2-7

shows these three parameters

monitored for four different

subjects. This work shows that

the Kinect can be used to

monitor a person's gait in the

home with no wearable

sensors in an unobtrusive way.

The chief weakness with this

method is the susceptibility to

obstructions and the quality

constraints necessary to ensure

good results.

Staranowicz et al. [90]

developed a system that would

help solve this issue, though

with the extra complexity of

this system it is not an ideal walking speed (in/sec)

stride length (in)

— stride time (sec)

APARTMENT 1 - RESIDENT 1

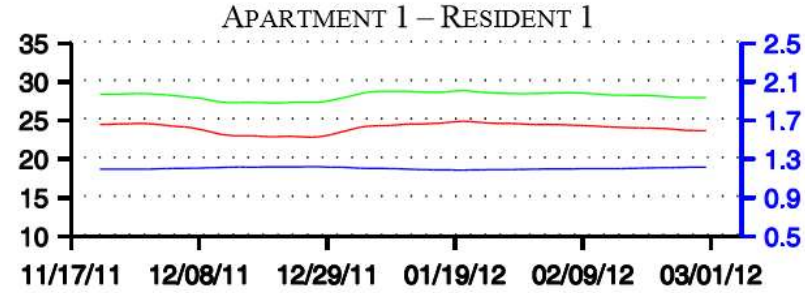

APARTMENT 2 -RESIDENT 1

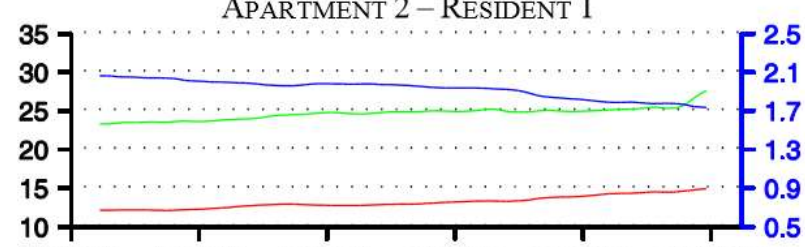

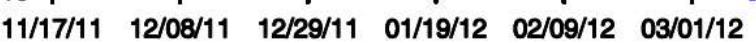

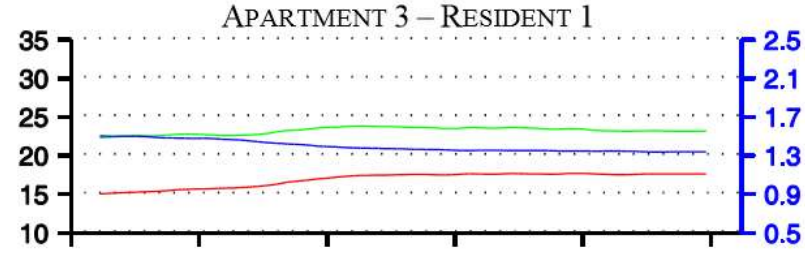

$\begin{array}{llllll}11 / 17 / 11 & 12 / 08 / 11 & 12 / 29 / 11 & 01 / 19 / 12 & 02 / 09 / 12 & 03 / 01 / 12\end{array}$

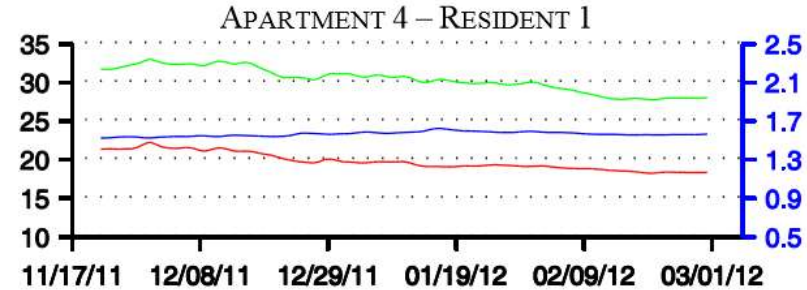

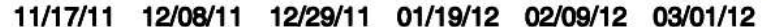

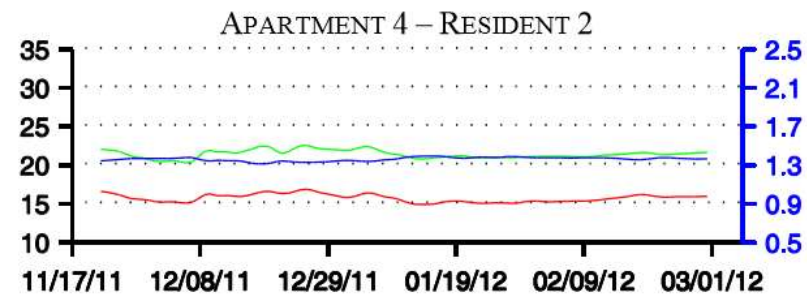

Fig. 4. Trends for the habitual, in-home gait parameters of walking speed, stride time, and stride length for five residents of four apartments computed using data from a four month period, Nov. 1, 2011, thru Feb. 29, 2012. The scale on the left applies to walking speed and stride length; while the scale on the right applies to stride time.

Figure 2-6: Trends for in-home gait parameters. From Stone et al. [8]

solution. The group uses the skeletal output from the Kinect system to monitor joint movement and, by extension, step time (left and right) and step length (left and right). One novel aspect of 
the system is the Kinect depth camera is mounted on an iRobot Create platform. The iRobot platform has been programmed to follow the subject "at a safe distance". This allows the system to monitor the subject, theoretically, wherever the iRobot platform can follow.

While it was not mentioned as a motivation, the robot/camera may help manage environments with obstructions. The researchers limited testing of the robot/camera platform to straight line walking paths, so it is unclear how well the platform will function as the viewing angle to the subject changes direction and the robot scoots around obstructions. Depending upon the technology used to follow the subject being monitored, it is also conceivable that the robot may "get lost" in a cluttered apartment, leaving the subject unmonitored and, likely, requiring some intervention.

\section{Summary}

In the last several sections, a wide range of different techniques currently in use to monitor gait have been explored. With a goal of monitoring gait in the home in an inexpensive and non-intrusive way, each of the approaches mentioned in this section meets these goals to some extent with the approach used by Stone et al. coming the closest. The motion capture systems such as the Vicon and GaitRite systems are extremely accurate but are both expensive and require a great deal of equipment to be installed - not feasible for a home environment. External sensors that can be built into carpets would create a system that would be more accepted in the home but, again, these would be cost prohibitive at least with today's technology. Cameras show more promise as they can generate results that are comparable with the motion capture systems but at a much lower cost. Cameras can, for the most part, be placed to minimize their visibility in the home. The Microsoft Kinect is proving to be a powerful tool to measure gait and, as is shown here, can produce results that are comparable to a motion capture system. The only drawback is the sensitivity of the approach to occlusions in the home. Most homes will typically 
have some furniture, (tables, chairs, etc...) in the middle of the room causing some of the subject's walks to be occluded by those objects from time to time. What is needed, then, is an approach that is less sensitive to obstructions.

\section{Clustering and Outlier Detection}

Possibilistic C-Means (PCM) Clustering Algorithm

The Possibilistic C-Means (PCM) clustering algorithm was developed by Krishnapuram and Keller in 1993 [91] as an improvement over the Fuzzy C-Means (FCM) clustering algorithm developed by Bezdek more than a decade prior. The crux of the improvement is the relaxation of the constraint that memberships among the various clusters are probabilities and must, therefore, sum to one. This relaxation allows the membership to more reflect the typicality or compatibility with a particular model.

The objective function for this approach is presented as:

$$
J_{m}(L, U)=\sum_{i=1}^{C} \sum_{j=1}^{N}\left(u_{i, j}\right)^{m} d_{i, j}^{2}+\sum_{i=1}^{C} \eta_{i} \sum_{j=1}^{N}\left(1-u_{i, j}\right)^{m}
$$

When the objective function is differentiated and solved for $u_{i, j}$, the following function results:

$$
u_{i, j}=\frac{1}{1+\left(\frac{d_{i, j}^{2}}{\eta_{i}}\right)^{\frac{1}{m-1}}}
$$

Where the value of $\eta_{i}$ is given by this equation: 


$$
\eta_{i}=K \frac{\sum_{j=1}^{N} u_{i, j}^{m} d_{i, j}^{2}}{\sum_{j=1}^{N} u_{i, j}^{m}}
$$

In all three equations, $\mathrm{L}$ is the set of clustering prototypes, $d_{i, j}^{2}$ is the distance from feature point $\boldsymbol{x}_{j}$ to the $i^{\text {th }}$ prototype, $\mathrm{C}$ is the total number of classes, $u_{i, j}$ is the typicality of the $j^{\text {th }}$ point to the $i^{\text {th }}$ prototype, and $m$ is a weighting exponent which is greater than one.

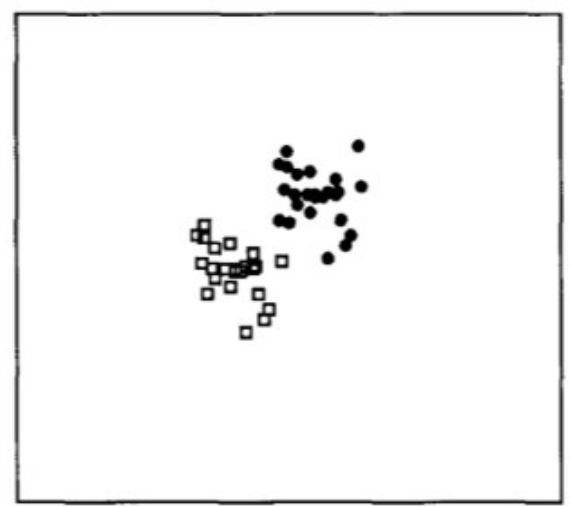

(a)

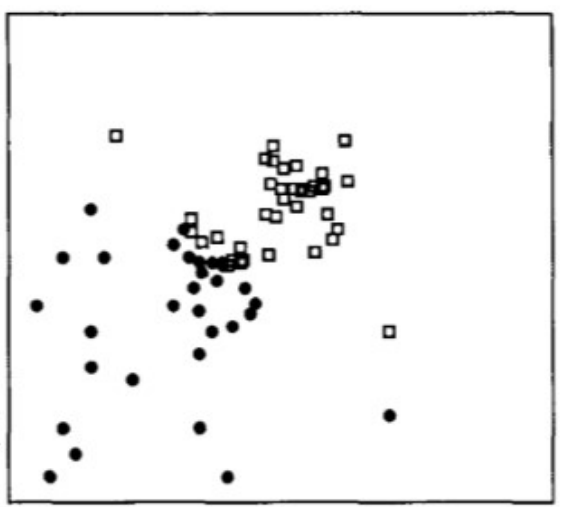

(c)

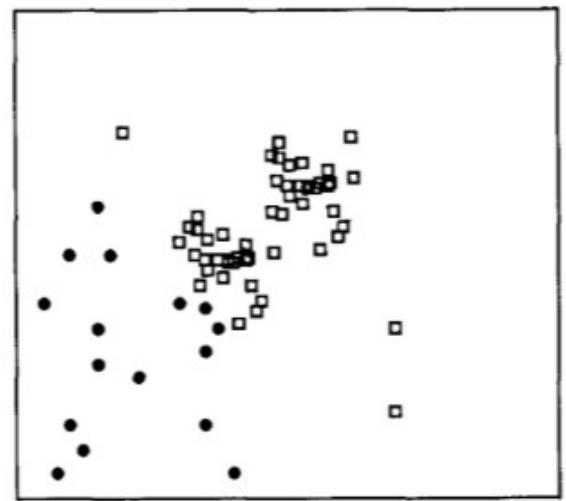

(b)

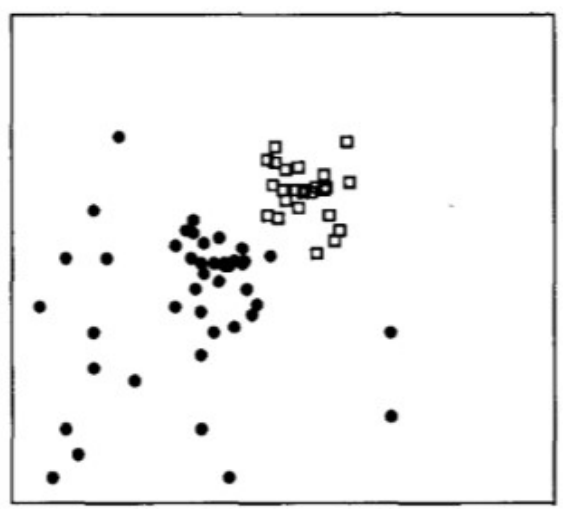

(d)

Fig. 4. Results on a data set generated by a Gaussian random number generator: (a) the crisp partition resulting from the HCM. FCM, and PCM algorithms; (b) the crisp partition from the HCM algorithm when noise is added; (c) the crisp partition from the FCM algorithm when noise is added; (d) the crisp partition from the PCM algorithm when noise is added.

Figure 2-7: Examples of clustering using HCM, FCM, and PCM algorithms. Plots $b, c$, and $d$ show the clusters in the presense of noise while (a) simply shows the clustering without any noise in the data. Note that the PCM algorithm (d) shows the best performance. From [91]. 
Figure 2-7 shows an example of the PCM, FCM, and Hard C-Means (HCM) algorithms being used to cluster data. The upper left image is a set of data points clustered by the HCM algorithm. Noise was then added to the points and each of the three algorithms was used to cluster the data. The HCM algorithm, which did well in the absence of noise, struggled once noise was added. The FCM also struggled with the noise, assigning a number of points in the lower left cluster to the upper right cluster. The PCM algorithm was, even in the presence of noise, able to correct cluster the data.

The authors mention that the algorithm does depend upon the initialization, particularly because the centers in the possibilistic algorithms do not move much so it is important to ensure that initial centers are somewhat close. Their recommendation is to utilize any hard or fuzzy clustering algorithm initially to approximate the cluster centers and use that information to initialize PCM clustering.

\section{Possibilistic Fuzzy C-Means (PFCM) Clustering Algorithm}

The PFCM algorithm, developed by Pal et al. [92], solves many of the challenges in both the FCM and PCM algorithms by marrying the two approaches into a single clustering algorithm. In the FCM, the fuzzy memberships for each point across all clusters must sum to 1 . This implies that even outliers must be assigned to a specific cluster which can influence the structure of the clusters. Relaxing this constraint, one of the goals of the PCM algorithm, however, allows outliers as well as cluster members to have very little influence on the model. This actually goes too far and makes this method highly "brittle" to choices in parameters as well as initialization.

Their solution was creating an optimization problem that combines both a fuzzy and a possibilistic component. While this was done earlier in the development of the FPCM algorithm, that approach had a flaw for large data sets for which the workaround was to scale up the 
typicality values. The PFCM algorithm corrects this problem for large data sets and creates a clustering algorithm that is less dependent upon initial conditions, and performs well in both estimating memberships and typicalities, as well as identifying reliable cluster centers.

Figure 2-8 shows the three equations to implement this algorithm. The $\mathrm{D}$ terms are the distances between each data point and each cluster center. The $\mathrm{m}$ and $\eta$ terms represent constants set greater than 1.

The $a$ and $b$ terms define the relative importance of fuzzy membership and possibilistic

$$
\begin{array}{rl}
u_{i k} & =\left(\sum_{j=1}^{c}\left(\frac{D_{i k A}}{D_{j k A}}\right)^{2 /(m-1)}\right)^{-1} \\
1 \leq i \leq c ; \quad 1 \leq k \leq n & 1 \\
t_{i k} & =\frac{1+\left(\frac{b}{\gamma_{i}} D_{i k A}^{2}\right)^{1 /(\eta-1)}}{\sum^{\leq} \leq i \leq c ; 1 \leq k \leq n} \\
\mathbf{v}_{i} & =\frac{\sum_{k=1}^{n}\left(a u_{i k}^{m}+b t_{i k}^{\eta}\right) \mathbf{x}_{k}}{\sum_{k=1}^{n}\left(a u_{i k}^{m}+b t_{i k}^{\eta}\right)} \\
1 \leq & \leq i \leq c
\end{array}
$$

Figure 2-8: Equations for the PFCM algorithm. The first equation (21) computes the fuzzy memberships for each data point. The second equation (22) computes the typicality for each point, and the third equation (23) updates the cluster centers. These equations come from [81]

typicality terms in the clustering. If the constant a is set to zero, then the algorithm behaves as a fuzzy c-means algorithm. Setting the constant b to zero, in turn, causes the algorithm to behave like the PCM algorithm.

The authors conclude that this approach combines the best features of both fuzzy and possibilistic clustering while solving the problems identified in previous algorithms. This method generates three sets of data. The first is the prototype cluster centers. The second is the set of fuzzy membership values (the " $u$ " terms mentioned above) and the third are possibilistic typicality values (the " $\mathrm{t}$ " terms above). While these $\mathrm{u}$ and $\mathrm{t}$ data sets are not identical to their respective counterparts in FCM and PCM, they do share the same properties and, consequently, can be used 
similarly. The $a$ and $b$ terms can be used to adapt the algorithm to behave more like a PCM or FCM algorithm in instances where better estimation of centroid or membership is needed.

\section{Change Detection using One Class Possibilistic C-Means (OCPCM)}

Wang used One Class PCM to detect outliers in data representing potential health anomalies [93]. Using several different sources of data, the goal was to isolate anomalous readings as indicative of potential health problems. To accomplish this, a set of data was clustered using the PCM algorithm to compute typicality values for a single cluster prototype. PCM was chosen as the cluster prototype is less affected by outliers. As these outliers are removed, very little movement of the cluster center is expected. Contrast this with a larger amount of expected movement if true members of the cluster are removed.

This behavior is the key to this technique. The entire dataset is clustered using the OCPCM algorithm. Next, the data point with the lowest typicality value is removed from the dataset. The set is clustered once again and the difference between the centers of the two prototypes is computed. This is repeated removing a point and recalculating the prototype for each pass. This is repeated until the change in the prototype center is sufficiently large.

As can be seen in Fig 2-9, the distance moved by the prototype centroid move very little over the first 40 days after which a steady increase in movement is seen. It is at this point, around day 40 , that the last outlier is removed, and actual members of the cluster are removed. 
In the study, Wang

used this approach to identify outliers in data

streams from several

sources to identify changes

which may portend clinical

concerns. This approach

Figure 2-9: Graph of movement of the cluster center during a One Class PCM computation. Note that around day 40 , the prototype center begins to

change more dramatically indicating the removal of points within the cluster. From [82].

showed some success in

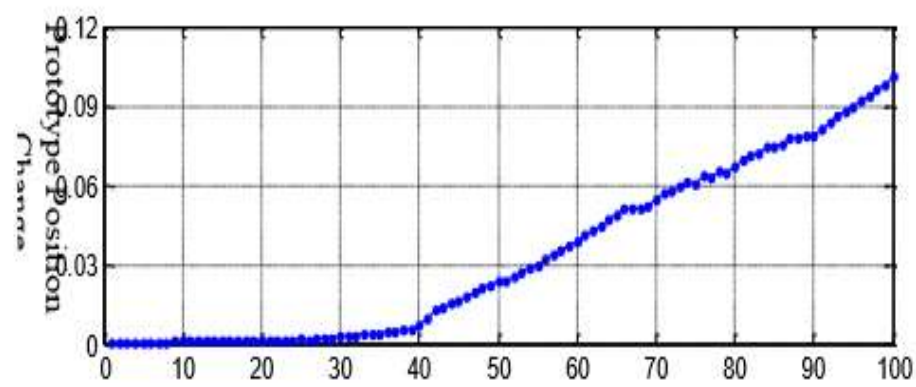

predicting events. The study showed more difficulty in predicting falls as many falls are purely random events for which there may not be a visible trend leading up to the event. 


\section{Chapter 3 Metrics from In-Home Sensor Data to Assess the Effect of}

\section{Clinical Therapies}

This chapter contain the text from the above paper which has been published in the SmartHealth Journal. The paper presents, in detail, the development, verification, and validation of the metrics against lab data and in home data. It also validates the performance of the metrics against existing fall risk assessments for several subjects. Lastly, it uses all three classes of metrics to explore the weighted vest therapy on three subjects.

Please note that the references section of the paper has been removed and all references have been updated to refer to the "References" section at the end of the dissertation. All figures, tables, and equation references in this chapter refer to figures, tables, and equations in this chapter alone.

Please also note that the following table shows the mapping between the patient number in this paper and the patient numbers shown in main dissertation paper.

\begin{tabular}{|l|l|}
\hline Patient \# in this chapter & $\begin{array}{l}\text { Corresponding Patient } \\
\text { \# in whole dissertation. }\end{array}$ \\
\hline 1 & 3004 \\
\hline 2 & 3046 \\
\hline 3 & 3013 \\
\hline 4 & 3050 \\
\hline 5 & 3037 \\
\hline V1 & 3012 \\
\hline V2 & 3057 \\
\hline V3 & 202 \\
\hline
\end{tabular}




\title{
Metrics from In-Home Sensor Data to Assess Gait Change Due to Weighted Vest Therapy
}

\author{
R. Wallace ${ }^{1}$, C. Abbott ${ }^{2}$, C. Gibson-Horn ${ }^{3}$, M. Rantz ${ }^{4}$ and M. Skubic ${ }^{5}$
}

Abstract - A set of metrics and a methodology were developed to characterize a subject's ability to ambulate.

These metrics use the movement of the subject's centroid as detected by an inexpensive depth camera system. The centroid is chosen as it is less sensitive to cluttered environments typically found in a person's home. Three classes of metrics focusing on three major categories of motion were developed. The first class measures fundamental characteristics of movement in three directions. The second class focuses on measuring the walk's entropy. The third class uses periodicity in the subject's motion to deduce temporal gait parameters including stride length. Metrics are validated and compared to existing Fall Risk Assessments (FRA's). While results show strong correlation to many FRA's, not every subject has the same relationships between metrics and FRA's suggesting a unique "fingerprint" of metrics associated with a subject and/or their condition.

The methodology was tested using a group of subjects undergoing Balance Wear Therapy targeting sensory inputs to improve balance control. The ability of the metrics to detect changes in the subject's ambulation when the vest is either put on, or taken off was also explored. Results show sufficient sensitivity to detect changes when the vest is donned and doffed. Many effects are not seen immediately, but over 2-4 hours following donning or doffing the vest. Results also demonstrate the ability, using the size of the analysis window, to focus on the time required for the effects of each metric to change.

\section{1 - Introduction}

\footnotetext{
The research is funded by the NSF under grant CNS-0931607

${ }^{1} \mathrm{RW}$ is with the Electrical Engineering and Computer Science (EECS) Department, University of Missouri, Columbia, MO. (rswallace@missouri.edu) (Corresponding Author)

${ }^{2}$ CA is with the Department of Physical Therapy, University of Missouri, Columbia, MO.

${ }^{3} \mathrm{CGH}$ is a researcher in the Department of Physical Therapy, Samuel Merritt University, Oakland, CA, and a co-owner of Motion Therapeutics.

${ }^{4}$ MR is with the Sinclair School of Nursing, University of Missouri, Columbia, MO.

${ }^{5}$ MS is with the EECS Department, University of Missouri, Columbia, MO.
} 
More than one third of older adults fall each year [1]-[4]. Of these falls, $10 \%$ to $20 \%$ cause moderate to severe injuries. Of these falls, about three percent will result in a fracture of some kind [6]. According to the United States Centers for Disease Control and Prevention, in 2012, the direct medical cost of falls among older adults, adjusted for inflation, was over $\$ 30$ billion [94].

Beyond the physical and immediate financial cost of falls, there are longer term costs, psychological, physical and financial, that result from falling. Howland reported that the fear of falling may, in fact, affect social interaction regardless of actual fall risk [7]. Nevitt found that approximately one quarter of falls cause the subject to limit their normal activities, usually because of injury, but often simply due to the fear of falling again [6]. Reducing the occurrence of falls, then, can go a long way in reducing the direct and indirect costs of falls. Not only will the patient's life be extended, and direct hospitalization costs be reduced, but more patients will be able to maintain their functional independence longer, delaying or eliminating their dependency on family, friends, or the healthcare system as a whole. This will lead to a higher quality of life.

To better monitor the population of older adults in order to identify the potential of falling before it happens or between exams, a set of metrics was developed that can be used to study the effects of therapies and monitor a subject's gait as the subject carries out their typical day to day activities. Since the target is the home environment, we focused on using inexpensive depth cameras, such as the Microsoft Kinect ${ }^{\circledR}$.

In order to test these algorithms and metrics, we chose to use BalanceWear Therapy. BalanceWear Therapy (BWT) consists of strategically placing sensory inputs (small $1 / 4$ to $1 / 2$ pound weights) at various locations on the trunk. BWT is currently being utilized to treat patients with Multiple Sclerosis, Parkinson's disease, traumatic brain injury, vestibular issues, and ataxia. The BalanceWear ${ }^{\circledR}$ vest, produced by Motion Therapeutics in Oxnard, CA, consists of a vest, worn on 
the torso with Velcro areas where small, light weights are attached to the inside of the vest. The physical therapist performed a BalanceWear assessment to identify directional imbalance of a subject and strategically placed $1 / 4$ to $1 / 2$ pound increments of weight in specific locations on the vest to improve directional balance and postural control. The patient was then instructed to wear the vest for several hours once or twice during the day. Studies conducted have focused on the clinical effects of the vest [34]-[36] but have not investigated how the vest specifically impacts the patient's ambulation beyond gait speed.

A brief discussion of background and related work is included in Section 2. The Methods section describes how the data were collected and analyzed. The Metrics section discusses how each metric is computed. The Results and Discussion sections highlight the findings.

\section{2 - Related Work}

\section{1 - Fall Risk Assessments}

\subsection{1 - Timed Up-and-Go}

The "Timed Up and Go" (TUG) test is a modification of the "Get Up and Go" test developed by Mathias et al., 1986 to add a temporal component to the assessment [21]. Their research has shown that the TUG test is reliable, correlates well with the Berg Balance Scale, Gait Speed, and Barthel Index of "Activity of Daily Living", and appears to predict the patient's ability to go outside alone safely [21]. Other researchers have arrived at similar conclusions [22], [23].

The TUG is a timed test requiring the subject to rise from a chair, walk 3 meters, turn around, return to the chair, and sit back down.

Morris et al. [26] investigated the reliability of this measure in people with Parkinson's disease.

The TUG has been studied with other pathological conditions as well, including amyotrophic lateral sclerosis [27], post stroke [28], and orthopedic disturbances [29], [30]. 


\subsection{2 - Habitual Gait Speed}

Habitual gait speed (HGS) has been studied as a means to detect a significant change in a person's gait [31][32][33] which is a predictor of falls. To measure, the subject is asked to walk at a comfortable, habitual speed for a specified distance (20 feet is typical). The walk is timed and the average speed is computed. Bohannon found gait speed to be highly reliable [31]. This FRA was also shown to be moderately correlated with the subject's age $(-0.558 ; p<0.001)$, and minimally correlated with height and several mechanical aspects of gait (ankle dorsiflexion strength, hip flexion strength, and hip abduction strength). Knee extension strength was found to be strongly correlated with HGS. Bohannon also published height normalized means and standard deviations for Habitual Gait Speed and Maximum Gait Speed [31].

\subsection{3 - Functional Reach}

A third common fall risk instrument is the Functional Reach Assessment. This test guides the patient through a series of reach tasks designed to gauge not only the flexibility, but also the ability of the patient to balance sufficiently to reach. This instrument was published in 1990 by Duncan et al. [16]. The instrument measures the difference between the arm's length and the maximum forward reach using a fixed base of support.

\subsection{4 - Berg Balance Scale}

The Berg Balance Scale (BBS) is a test of 14 different items and is used to monitor fall risk principally relying on assessment of the patient's balance. The 14 activities that comprise the instrument evaluate the subject's balance, ability to rise from and sit on a chair, walking, turning, and balance while walking. In this instrument, all 14 items are rated on a scale of 0 to 4 corresponding from no ability to full ability. The final score is simply the total of each rating out of a total of 56 with each item being equally weighted [18]. 


\subsection{5 - Short Physical Performance Battery}

The Short Physical Performance Battery (SPPB) test [95] is commonly used to assess lower extremity strength and has been shown to have some correlation to recurrent falls [96]. The instrument measures five aspects of physical performance and scores each in the range of [0..4] where a score of 0 represents the inability to complete the task and a score of 4 indicates the highest level of performance [95].

\subsection{6 - Single Leg Stance}

The single leg stance (SLS) assesses fall risk by examining the subject's balance. In this instrument, the subject is asked to stand on one foot with the second foot raised slightly (near the ankle of the stance foot). The time the subject is able to maintain balance is recorded. This is done three times with eyes open and three times with eyes closed. The six trials alternate between eyes opened and closed and a 5 min. rest period was allowed between each pair of trials. Shorter stance times suggest a higher risk of falling [97].

\section{2 - Studies with Strategically Weighted Vest}

While there is published research being conducted on the use of strategically weighted vests in other applications, there has been little research on the usefulness of these vests for improving the ambulation and reducing the risk of falls in the elderly. In [34], Gibson-Horn presents the case of a single patient who experienced improved balance and gait during static and dynamic activities. In [35], Widener et al. expand upon the previous study to include 16 subjects with Multiple Sclerosis and found significant improvement in several clinical assessments of balance. The last study [36] was a full clinical trial demonstrating immediate improvement of gait velocity and functional activity. Hunt et al. used a force plate in an attempt to deduce how balance is affected by BBTW [98]. More recently, a study [9] has demonstrated the ability to measure 
changes in some metrics in the home environment. This paper more fully develops these proposed metrics and presents new metrics to serve as a basis to study the mechanisms by which the vest and other interventions improve a subject's ambulation.

\section{3 - Wearable Sensors}

Several techniques have been utilized to measure gait parameters in a laboratory or clinical setting. These may rely on markers worn by the subject that are detected by some form of sensor external to the patient or by some form of sensor (usually an accelerometer) worn by the subject. The Vicon ${ }^{\circledR}$ Motion Capture System is an example of the first type. This system requires multiple infrared reflecting markers to be worn by the subject, typically at the joints, in order to capture a skeletal model. Using several infrared emitters and sensors, the position of each of the markers is captured and tracked while the subject is asked to perform various tasks.

While this system is extremely accurate in monitoring the subject's position and works well in a laboratory or clinical environment, it is prohibitively expensive and invasive to use for home monitoring as it requires multiple infrared cameras and emitters to be installed in the subject's environment. Additionally, the markers must be worn at all times for monitoring to occur.

An alternative, and less expensive, solution is achieved using sensors worn by the subject. This common approach [39]-[42] requires the subject to wear one or more sensors which wirelessly transmits the subject's acceleration in each of three directions, or other related parameters, to a computer which can then deduce location and speed. While the accuracy of this technology is lower than with the Vicon ${ }^{\circledR}$ system, this type of system has been used to determine ambulation parameters. These systems tend to be much more affordable as the sensors are inexpensive and the computational power required to process these data is not great. New sensors are also emerging which are incorporated into clothing or footwear, which may be less obtrusive [99]. 
The drawback of this type of system is the necessity of wearing a sensor and typically, recharging the device. For a subject to reliably wear the sensor, it must be designed to be put on, taken off, recharged, and activated easily. The subject must also be wearing the sensor when they are moving throughout their environment. If the subject were to wake in the middle of the night, they may not take the time to put on the sensor, leaving them unmonitored during a period when they are at a higher risk of falling. Studies also show that many seniors refuse to wear wearable sensors or are unable to operate them [100], [101]. Lastly, there are psychological reasons why these wearable sensors may not be accepted by the elderly population which can range from simply being unwilling to wear the device, questioning the need for the device, or even associating the device with an admission of vulnerability [44].

\section{4 - Non-Wearable Sensors}

What is more practical for today's vulnerable seniors, particularly for continuous in-home monitoring, is an inexpensive system that does not require any direct interaction by the user. Several approaches have been used including single camera [102], multiple orthogonal webcams [103], [49], [104], and Doppler radar to monitor subject movement and or falls [69]. An inexpensive depth camera, such as the Microsoft Kinect system, has been used to measure gait parameters. This approach uses the movement of the subject's feet to measure stride length, stride time and walking speed [66], [105], [68], [106], [107], [108], [109]. While this system generates good results as compared to ground truth generated by a Vicon ${ }^{\circledR}$ Motion Capture System, it suffers when the subject's feet are occluded by objects in the subject's environment (tables, chairs, etc....). In this paper, we investigate a set of metrics that can be computed using centroid values extracted from depth images taken by an inexpensive depth camera system. The remainder of this paper describes the development, validation, and use of such metrics. 


\section{3 - Methods}

\section{1 - Centroid Measurement}

One solution to the problem of occluded legs and feet is to monitor the centroid of the subject's image. As a person ambulates, there is oscillation in the vertical direction when the heel plant is made at the start of a new step to the maximum when the opposite leg is in the middle of its swing. Similarly, there is oscillation in the lateral direction as the subject's center of mass shifts over the planted foot to balance the subject when the swinging foot is lifted. Since the subject's centroid is easier to identify and less likely to be occluded, we hypothesize this would improve the ability to measure in an unstructured and cluttered environment.

\section{2 - Data Collection}

After receiving approval from the University of Missouri Institutional Review Board, a Microsoft Kinect system was placed in the apartments of four subjects. Subjects were chosen based upon their current physical condition and likely benefit of the BBTW strategically weighted BalanceWear Therapy. Depth image data are used directly as in previous work [8], [110]. The system collected and stored the centroid location at 8 to 30 frames per second for each object that moved throughout the room.

The camera system was active 24 hours per day in the living room of each apartment, and any walks made by the home resident or any visitors during that time were captured. Data were captured for roughly 220 to more than 500 days, depending upon the subject. Walks by people other than the subject are classified as visitors and culled as described in the next section.

\section{3 - Data Analysis}

The Kinect system was used to collect the raw depth image data, and the dynamically updated background was removed. The foreground image was segmented, and a 3-D point cloud was 
generated, representing the person moving about the scene. The centroid values were computed from this point cloud.[66]. Since the data were collected in a subject's unstructured and cluttered apartment, and not a laboratory setting, the walks were furthered filtered by culling all walks that were not considered purposeful. Purposeful walks are defined as walks at least 1.22 meters long, speed at least $12.7 \mathrm{~cm} /$ second, with a duration of at least 1 second [65].

For this study, the centroid data were adjusted to a constant rate of 15 frames per second (averaging multiple points and interpolating missing points). The data were then transformed from a room oriented location $(x, y, z)$ to a triplet of error values $\left(\delta_{x}, \delta_{y}\right.$, and $\left.\delta_{z}\right)$ corresponding to the deviation from the expected location $\left(x^{\prime}, y^{\prime}, z^{\prime}\right)$ along the walking path. The expected location was found by projecting a best-fit line over one second of data, centered on the current point in time, and extending it to the next frame. By convention, the $X$ direction was chosen to be in the direction of travel, the $Y$ direction was lateral, and the $Z$ direction was vertical. The positive directions for $\mathrm{X}, \mathrm{Y}$, and $\mathrm{Z}$ indicate forward, left, and up, respectively. This process is illustrated in fig. 3-1.

The last pre-processing step removes those samples that are not likely to be generated by the subject. To do this, a Gaussian mixture model (GMM) clustering algorithm was applied using height and speed as features. The GMM is initialized randomly and 50 models are computed for $\mathrm{N}+1$ clusters where $\mathrm{N}$ represents the number of residents in the apartment. The Fowlkes-Mallows score is computed for each model and

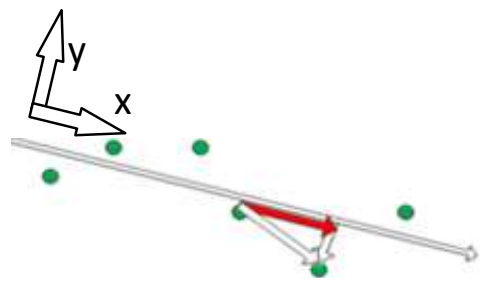

Figure 3-1: Diagram of the process used to generate the $\left(\delta_{x}, \delta_{y}\right.$, and $\left.\delta_{z}\right)$ error signals from the room coordinates $(x, y, z)$. The $X-Y$ plane is shown (as if looking down from the ceiling). The dots represent the measured position. The long arrow represents the 1 second average path centered on the current position. The red (dark) arrow represents the expected position at the next sample. The error in the $X$ direction is simply the difference between the expected position and the component of the actual displacement. The shortest arrow represents the error in the $Y$ direction and is the component of the error along a line perpendicular to the expected path. The error in the $Z$ direction is simply the difference between the $z$ component of the centroid and the average centroid height 
the model with the best score is selected as the winner. The data in the smallest cluster is discarded as belonging to visitors. Lastly, for apartments with two residents, the cluster for each resident is differentiated using their respective heights. This is similar to the approach used in Stone et al. [105].

4-Metrics

\section{1 - Basic Metrics}

After pre-processing, the following metrics are computed using the error values generated ${ }^{6}$.

- Asymmetry is computed in all three directions as the ratio between the mean error $\left(\overline{\delta_{l}}\right)$ and the maximum error $\left(\delta_{i}\right)$ for a given walk $(i=x, y$, or $z)$. This measures the degree to which the walk favors one side versus the other.

$$
A_{i}=\frac{\bar{\delta}_{l}}{\operatorname{MAX}\left(\left|\delta_{i}\right|\right)}
$$

- Peak-to-Peak (p2p) is computed in all three directions as the difference between the largest error values in both the positive and negative directions.

$$
p 2 p_{i}=\operatorname{MAX}\left(\delta_{i}\right)-\operatorname{MIN}\left(\delta_{i}\right)
$$

- Walk speed is computed by dividing the distance covered by the duration of the walk (T). The variable $\mathrm{n}$ represents the number of samples for the walk.

$$
S=\frac{\sum_{i=1}^{n-1} \sqrt{\left(x^{\prime}{ }_{i+1}-x^{\prime}{ }_{i}\right)^{2}+\left(y^{\prime}{ }_{i+1}-y^{\prime}{ }_{i}\right)^{2}}}{T}
$$

- The ten foot walk metric is computed utilizing the total actual distance moved by the centroid (D) and the duration of the walk(T).

\footnotetext{
${ }^{6} \mathrm{~A}$ table listing the variable names and meanings for this article is provided in Appendix 1.
} 


$$
W=T \times \frac{10 \mathrm{ft} .}{D}
$$

- Walk Efficiency is computed as the ratio between the most direct walking path $\left(x^{\prime}, y^{\prime}\right)$ and the actual path $(x, y)$ using the following formula:

$$
E=\frac{\sum_{i=1}^{n-1}\left(\sqrt{\left(x^{\prime}{ }_{i+1}-x^{\prime}\right)^{2}+\left(\left(y^{\prime}{ }_{i+1}-y^{\prime}{ }^{\prime}\right)^{2}\right.}\right.}{\sum_{i=1}^{n-1}\left(\sqrt{\left(x_{i+1}-x_{i}\right)^{2}+\left(\left(y_{i+1}-y_{i}\right)^{2}\right.}\right.}
$$

\section{2 - Entropy Metrics}

Entropy measures the uncertainty, irregularity, or randomness in a given signal. In this case, entropy will be used to measure the irregularity in the subject's gait. The hypothesis is that a healthy individual's gait is characterized by more regular, sinusoidal, movement laterally and vertically. As a subject's gait degrades due to age or disease, the gait can become more irregular. This would cause entropy measurements to increase as the subject's gait degrades indicating a higher risk of falling. Arafat et al. used entropy to study gait deficiencies and identify ataxia in horses [111]. In his work, he utilized three different computations of entropy. The first, equation 6, by Deluca and Termini in 1972 [112], is given by:

$$
H_{D T E}^{\delta_{K}}=-K \sum_{j} \delta_{k j} \log _{2} \delta_{k j}+\left(1-\delta_{k j}\right) \log _{2}\left(1-\delta_{k j}\right)
$$

Equation 7, developed by Pal in 1989 [113] is given here:

$$
H_{P P E}^{\delta_{k}}=K \sum_{j} \delta_{k j} e^{1-\delta_{k j}}+\left(1-\delta_{k j}\right) e^{\delta_{k j}}
$$


And equation 8, developed by Pal \& Bezdek in 1994 [114]:

$$
H_{\alpha Q E}^{\delta_{k}}=K \sum_{j} \delta_{k j}^{\alpha}+\left(1-\delta_{k j}^{\alpha}\right)^{\alpha}
$$

In all three formulae, $\delta_{k j}$ is the error signal in a particular direction. $\mathrm{K}$ is a normalization constant taken to be 1 in each of these measurements.

The entropy metrics determine the randomness or unpredictability of the subject's walks. In a healthy younger person, examining the movement of their centroid in the $\mathrm{Y}$ direction (lateral to the direction of motion) would show a fairly regular sinusoid as the centroid moves back and forth to keep the subject's center of mass above the planted foot. For a subject with difficulty balancing, as an example, there may be some additional random movement due to unsteadiness. It is this extra randomness that this metric will measure. For a regular, more sinusoidal-like signal, the entropy will be low. For a more irregular, random centroid path, the entropy will increase.

\section{3 - Temporal Metrics}

This estimation exploits the periodic nature of the error value $\delta_{z}$ during a typical walk. During a normal stride, the centroid reaches a vertical minimum value when both feet are in contact with the ground and reaches a maximum when either foot is in the middle of its swing and closest to the other leg. By measuring the period of this oscillation, the average stride time for a particular walk can be deduced.

The first step is to separate the components of centroid movement that most strongly captures the periodic components of a walk. In particular, we want to focus on the sudden change in vertical acceleration and velocity coincident with the foot plant. To isolate this, the vertical 
centroid signal is filtered using a $5 \mathrm{~Hz}$ high pass digital filter to remove low frequency components. The signal is then passed through a three-point triangular filter shown here:

$$
\delta_{z}^{\prime}(i)=\frac{\delta_{z}(i-1)+2 \delta_{z}(i)+\delta_{z}(i+1)}{4}
$$

During development, it was found that for steps that do not include crisp foot plants, the accuracy of the algorithm decreases. While the lateral component of a typical healthy walk should resemble a sinusoid, the vertical component should more closely resemble a sequence of arches, more like $|\sin (x)|$ than $\sin (x)$. To isolate these walks, the Deluca and Termini entropy equation (eq. 6) was used to compute the entropy of the vertical component of the centroid movement. Through experimentation, it was found that if walks do not score an entropy value in the range of [1..10], they will generate a large amount of error in the stride time value. Consequently, these walks with an entropy value outside of this range are discarded.

Lastly, a Fast Fourier Transform (FFT) is taken of the filtered signal. The period corresponding to the highest peak that is greater than zero is selected. Stride time is defined as the average time between the same events (for example, right foot heel strike) in two successive strides. Stride time, then, is computed by doubling the average step time (reciprocal of the dominant frequency in the FFT).

Once stride time is computed, the left and right steps are isolated using the following algorithm:

1. Compute the vector, $\mathbf{M}$, containing the time indices of all of the local minima in the $\delta_{z}$ signal.

2. Compute the vector, $\mathbf{P}$, containing the time indices of all of the local maxima in the $\delta_{z}$ signal.

3. Compute Step Time on both sides of the walk: 


$$
\begin{aligned}
& S T 1=\frac{1}{n / 2} \sum_{i=O D D}^{n-1} \boldsymbol{M}(i+1)-\boldsymbol{M}(i) \\
& S T 2=\frac{1}{n / 2} \sum_{i=E V E}^{n-1} \boldsymbol{M}(i+1)-\boldsymbol{M}(i)
\end{aligned}
$$

4. Compute Left Step Time and Right Step Time

a. If $\left(\delta_{y}\left(\frac{M(2)-M(1)}{2}\right)>0\right)$

i. $\quad$ Left Step Time $=$ ST1

ii. $\quad$ Right Step Time $=$ ST2

b. else

i. $\quad$ Left Step Time $=$ ST2

ii. $\quad$ Right Step Time $=$ ST1

Bounce is then computed using the following formula:

$$
B=\operatorname{MEAN}\left(\delta_{z}(\boldsymbol{P})\right)-\operatorname{MEAN}\left(\delta_{z}(\boldsymbol{M})\right)
$$

Trunk sway is computed by applying steps 1,2 on the $\delta_{y}$ signal then computing trunk sway using Eq. 13.

$$
S=\operatorname{MEAN}\left(\delta_{y}(\boldsymbol{P})\right)-\operatorname{MEAN}\left(\delta_{y}(\boldsymbol{M})\right)
$$




\section{4 - Minimum Detectable Change}

One use for these metrics is to study specific therapies to determine if there are any immediate or short term effects of a particular therapy. For example, one might want to study how putting on a set of shoe lifts impacts gait and ambulation. The clinician may want to monitor the subject as he goes about his daily activities without the lifts, then have him put the lifts on for a couple hours during the day. These proposed metrics could be used to study the effects on gait of the lifts, but some mechanism is needed to determine if the changes seen are statistically significant. Because the units for the proposed metrics vary, as does the number of walks for each particular subject, the concept of Minimum Detectable Change (MDC) was used. This value is computed from the standard deviations of the metrics both pre- and post-event. If the metric's value following the event deviates from the pre-event value by more than the MDC, we consider the change significant. MDC is computed using the approach defined in [115]. This calculation is skipped for pre-event distributions that are not Gaussian or if the pre-event signal is strongly correlated with the post-event signal $(\rho>0.50)$.

The computation is a two-step process using the following formulae. The first step is to compute the statistical " $\mathrm{t}$ " value using the following:

$$
\mathrm{t}=\frac{\overline{\mathrm{X}}_{\text {pre }}-\overline{\mathrm{X}}_{\text {post }}}{\mathrm{S}_{\mathrm{X}_{\text {pre }} \mathrm{X}_{\text {post }}} \sqrt{\frac{1}{\mathrm{n}_{\text {pre }}}+\frac{1}{\mathrm{n}_{\text {post }}}}}
$$

Where

$$
S_{X_{\text {pre }} X_{\text {post }}}=\sqrt{\frac{\left(n_{\text {pre }}-1\right) S_{\mathrm{x}_{\text {pre }}}^{2}+\left(n_{\text {post }}-1\right) S_{\mathrm{X}_{\text {post }}}^{2}}{n_{\text {pre }}+n_{\text {post }}-2}}
$$

and $\overline{\mathrm{X}}_{\text {pre }}$ and $\overline{\mathrm{X}}_{\text {post }}$ are the means for the pre-event distribution and the post-event distribution respectively. $n_{\text {pre }}$ and $n_{\text {post }}$ are the number of samples in the pre- and post-event distributions. 
$\mathrm{S}_{\mathrm{X}_{\mathrm{pre}}}^{2}$ and $\mathrm{S}_{\mathrm{X}_{\mathrm{post}}}^{2}$ are the variances for each distribution. Finally, MDC is computed using the following formula:

$$
M D C=t \sqrt{\frac{M S E}{n_{p r e}}+\frac{M S E}{n_{\text {post }}}}
$$

where MSE is the pooled mean squared error which is estimated by the variance for the pre-event distribution.

\section{5 - Pearson Correlation}

Pearson's Correlation was used to characterize the relationship between the behavior of the metrics and Fall Risk Assessment data. It measures the tendency for two variables to co-vary and has a range of $|\rho|=[0 . .1]$. A correlation value of 1 indicates perfect correlation between the two variables - as one changes, the other changes in the same direction. A correlation value of -1 indicates perfect negative correlation - as one changes, the other changes in the opposite direction. The formula for Pearson Correlation between two signals, s1 and s2, is shown in equation 17 [116]. It is worth noting that a strong correlation does not imply causality, merely that the two signals change along with, or opposite to, each other.

$$
\rho=\frac{\sum_{i=1}^{n}\left(\left(s 1_{i}-\overline{s 1}\right)\left(s 2_{i}-\overline{s 2}\right)\right)}{\sqrt{\left[\sum_{i=1}^{n}\left(s 1_{i}-\overline{s 1}\right)^{2}\right]\left[\sum_{i=1}^{n}\left(s 2_{i}-\overline{s 2}\right)^{2}\right]}}
$$

\section{5 - Measuring Effects of the Strategically Weighted Vest}

The vest, shown in fig. 3-2, was weighted in accordance with Motion Therapeutics' recommended Balance-Based Torso-Weighting (BBTW) assessment approach. The subject was evaluated for response to perturbation at shoulders and pelvis and small weights were chosen, along with the proper location for these weights. Following the assessment, the subject was instructed to wear the vest twice per day for two hours each session, though subjects often wore 
it for longer or shorter periods of time. With staff assistance, the subject recorded when the vest was put on or taken off. The BBTW assessment

was

repeated every two weeks and the amount were adjusted as needed. FRAs were also performed at this time.

For a given metric,
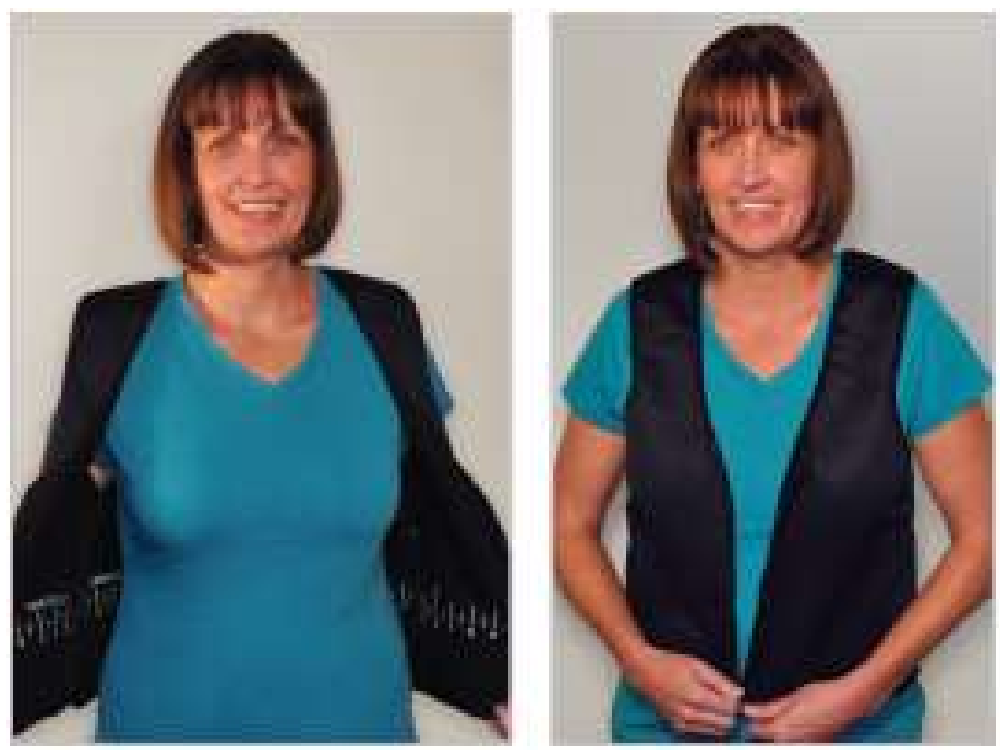

\section{of weight and locations}
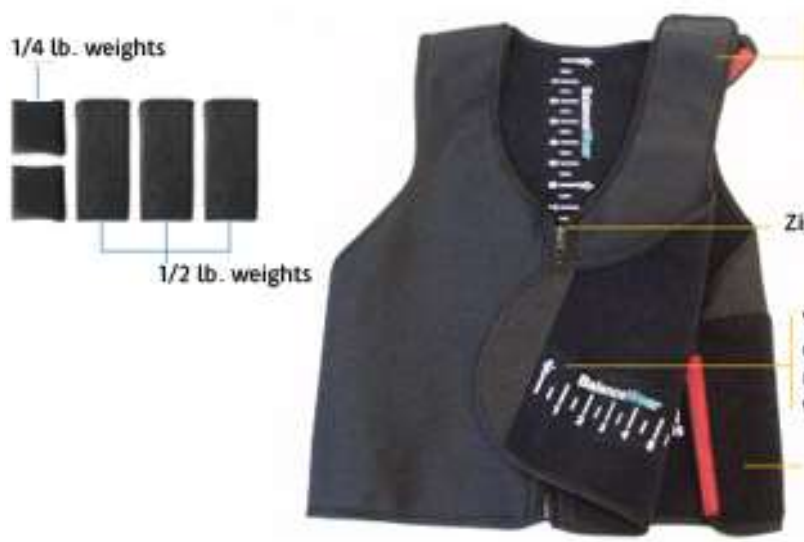

Adjustable shoulder straps

Zipper

Vest interior with convenient indicator marks for locating weight positions

Adjustabte side panels

Figure 3-2: An example of strategically weighted vest used in this study - the the time relative to BalanceWear BW300.

each time the vest is

donned ("vest on" event) or doffed ("vest off" event) was computed. These relative times were then binned into 40 bins and the average of each bin was computed. For this study, the width of the analysis window (\#bins $x$ bin width) was chosen for each subject to maximize the visibility of the vest's effects. This was done by scanning 50 different bin sizes from 0.375 minutes up to 18.75 minutes in 0.375 minute steps and selecting the window with the largest number of metrics showing a significant change. If the chosen bin width is too narrow, then the complete effect of the event will not be visualized as the range of the histogram will be smaller than the duration of 
the event's effects. If the chosen bin width is too (a)

wide, there will be some longer term effects that may hide the direct effects of the vest. This could occur if the effects of the event are short lived. The metric could change and return to its preevent value or to a new value within one or two bin widths. If this were to occur, then it may be difficult to detect the change. An alternative would be to set the window size to a fixed value in order to answer the question "Which metrics show changes within $x$ minutes of the

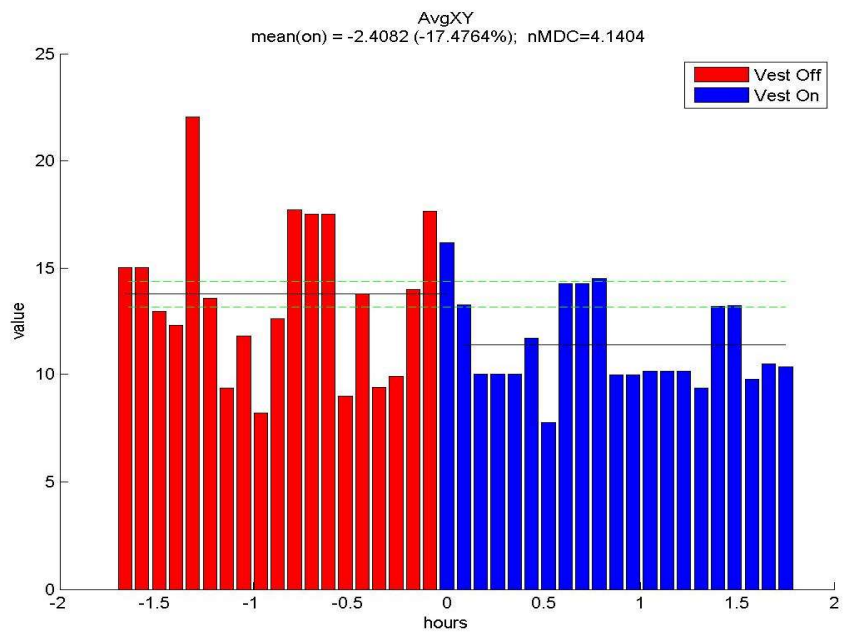

(b)

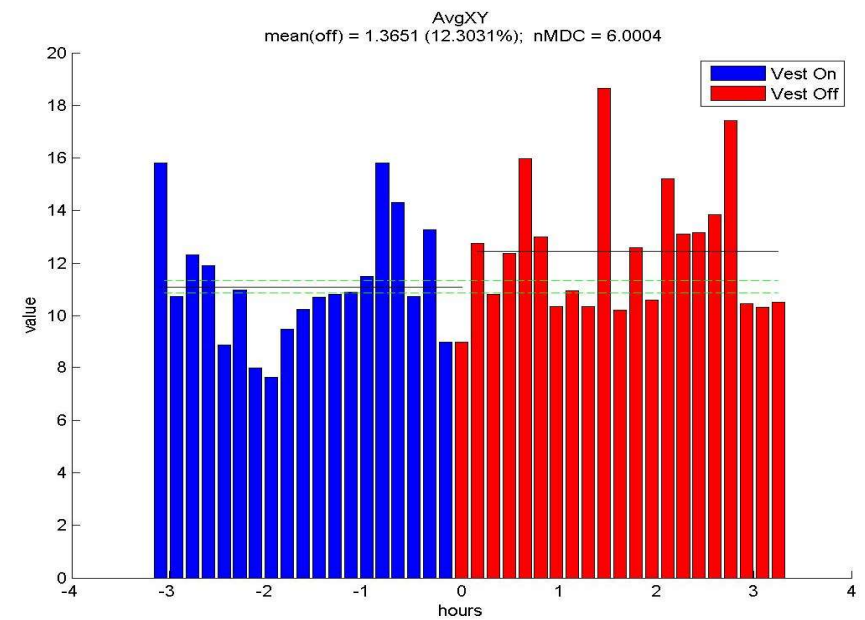

Figure 3-3: Plot showing Entropy in the XY direction for subject \#3004 centered on donning the vest (a) and doffing the vest (b). The plot shows the average step time (in seconds) of all walks that occurred at the time relative to the event shown on the $X$ axis. The solid, horizontal bars represent the mean value of the metric before and after the event. Dashed lines correspond to +/- 1 MDC based upon the pre-event value.

intervention?"

The average values of the metric in each bin were then plotted. A pair of sample plots is shown in fig. 3-3. The distributions both before and after the event were tested to determine if they are drawn from a Gaussian distribution using the t-test. The mean histogram value before the event 
is computed along with the MDC value. The mean value after the event is computed as well and the difference is compared to the MDC value to determine if the changes are significant.

\section{4 - Results}

\section{1 - Verifying and Validating Stride Time Estimation}

\subsection{1 - Lab Based Verification}

The proposed algorithm for estimating stride time was first compared to results obtained from the Vicon ${ }^{\circledR}$ system. The input data consisted of 13 healthy subjects each with 8 walks in our motion capture laboratory. Each walk was captured by 2 different Microsoft Kinect systems and each was filtered with two different algorithms. This resulted in a total of 416 different sets of walk data. As each subject had only 8 walks (each viewed by 2 cameras), walks from all subjects were combined and stride times and step lengths were computed by the Vicon ${ }^{\circledR}$ system and the proposed algorithm for each walk. The difference between the two results was computed and probability density functions were created. The arithmetic mean and the location of the peak in the PDFs were computed and compared. These are shown in figure 4.

The mean stride time for all walks computed by the Vicon ${ }^{\circledR}$ system was $1.13 \mathrm{~s}$ with a standard deviation of $0.101 \mathrm{~s}$. For the proposed algorithm, the mean was $1.11 \mathrm{~s}$ with a standard deviation of $0.123 \mathrm{~s}$. The mean error between the two systems was $0.02 \mathrm{~s}(2.2 \%)$ with a standard deviation of 0.160 . This shows similar results using both methods on the same data and shows equivalence in the lab. The PDF functions shown in figure 3-4 look very similar except for the dual peak around a stride time of 1.15 and a corresponding short and broad peak just to the left of 0.6 . 


\subsection{2 - Validation Using In Home Data}

A similar approach was applied using data captured from older adult subjects in their homes. The subjects used were all residents of TigerPlace, an independent living community located in Columbia, Missouri. Of the 7 subjects, 3 were male and 4 were female. Ages ranged from a high of 93 years old to a low of 75 years old with an average age of 86 . Note that only two of the subjects used for the validation portion of this study were also part of the weighted vest therapy study and are noted by the letter " $\mathrm{V}$ " in their designation. As it is not feasible to install a Vicon ${ }^{\circledR}$ system into each apartment, the algorithm developed and validated by Stone et al. [105] was used as the reference algorithm. The stride time from each suitable walk was computed and compared to the time computed by the reference algorithm for the same walk. This was done for 7 residents individually and the results

for each are shown in

Table 3-I. It can be seen that for all seven

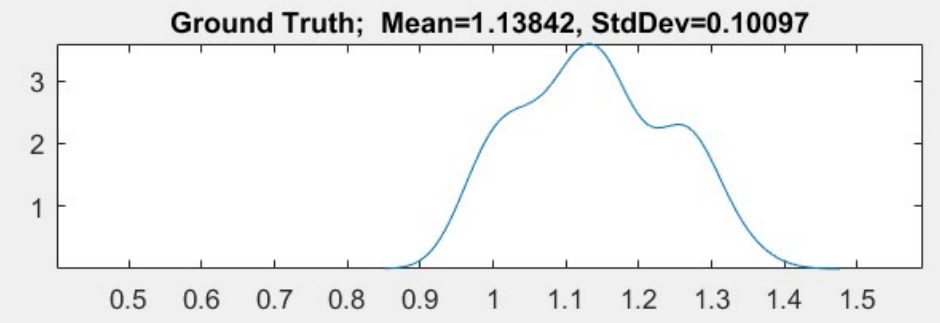

residents, the mean value

of the stride time error for each walk is less than

$0.3 \%$. This demonstrates

Centroid Estimate. Mean=1.11362, StdDev=0.12297

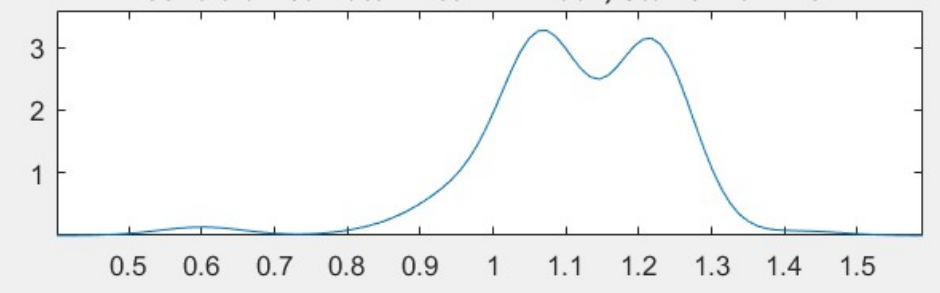

Error - Peak $=0.04 ;$ Mean $=0.02 ;$ StdDev $=0.16$

and validates the

proposed algorithm's

effectiveness

at

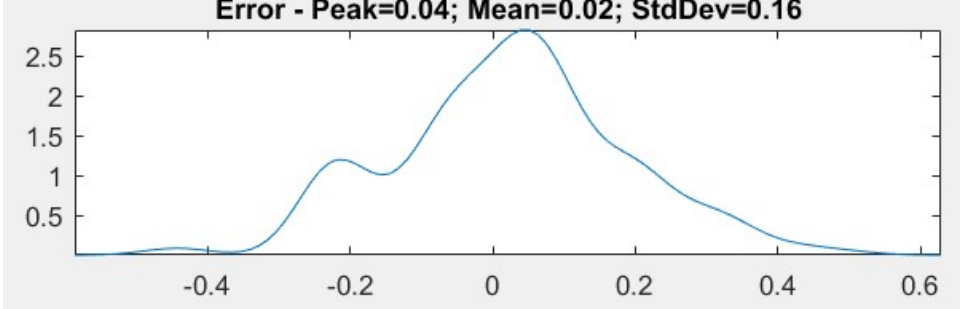

estimating stride time

based upon the

Figure 3-4: Laboratory validation of the Stride Time metric. The top graph shows the probability density function PDF for the stride time as detected by the Vicon ${ }^{\circledR}$ system. The middle graph shows the PDF for stride time as movement of the subject's computed by the proposed algorithm. The bottom graph shows the PDF of the error values between the two stride time measurements. 
centroid. The mean values for the errors in stride length and average speed for each resident, while not as accurate as stride time, are still within $7.5 \%$ and $2.6 \%$ of the reference algorithm respectively. These results also show that these algorithms correctly estimate stride length and speed.

Table 3-1: Results from validation of proposed algorithm using in-home data showing stride time, stride length, and walking speed. Mean Error is the mean of the probability density function of the error between the ground truth and proposed algorithms. Subject numbers starting with a "V" were included in the weighted vest study. Data includes all "purposeful" walks during the study which is variable from subject to subject but ranges from 220 to over 500 days.

\begin{tabular}{llll} 
Subj. & $\begin{array}{l}\text { Mean } \\
\text { Stride } \\
\text { Time } \\
\text { Error }\end{array}$ & $\begin{array}{l}\text { Mean } \\
\text { Stride } \\
\text { Length } \\
\text { Error }\end{array}$ & $\begin{array}{l}\text { Mean } \\
\text { Speed } \\
\text { Error }\end{array}$ \\
\hline 1 & $0.101 \%$ & $-1.93 \%$ & $-1.85 \%$ \\
2 & $-0.036 \%$ & $-7.23 \%$ & $-1.56 \%$ \\
3 & $0.001 \%$ & $-4.67 \%$ & $-1.98 \%$ \\
4 & $0.270 \%$ & $3.13 \%$ & $-1.38 \%$ \\
5 & $0.259 \%$ & $1.24 \%$ & $-2.18 \%$ \\
V1 & $0.214 \%$ & $-1.02 \%$ & $-2.52 \%$ \\
V2 & $-0.073 \%$ & $-6.04 \%$ & $-2.26 \%$
\end{tabular}




\subsection{3 - Validating to Fall Risk Assessment Instruments}

Next, we compared each proposed metric to the results of Fall Risk Assessments as well as the individual components of each FRA. The metric values on the three days centered on the day the FRA was conducted are compared against the actual FRA metric. Pearson correlation was performed between the individual metrics and FRA values for each resident individually. In these results, correlations that are not significant $(p>0.05)$ are not included. Table 3-2 shows those metrics that showed strong correlation $(|\rho|>=0.40)$ for one resident (\#1). Metrics related to step time (left, right, and total) have strong correlation with the residents FRA scores for HGS, BBS, TUG, SPPB, and SLS.

Looking at these results across other residents shows a different profile for each resident. This may suggest different disease progressions can amplify different relationships between metrics and their corresponding FRA's. To explore this, the number of strong correlations is tallied across all residents. The results of this analysis are shown in Table 3-3. While there are many pairs that

Table 3-2: Correlation Between Proposed Metrics and fra for Subject \#1.

\begin{tabular}{|c|c|c|c|c|c|c|}
\hline & Side Rea & hHGS & BBS & TUG & SPPB & SLS \\
\hline Speed & & & 0.5163 & -0.586 & & \\
\hline Ten Foot Walk & & & -0.5521 & & & \\
\hline Asymmetry X & & & 0.5582 & & & \\
\hline Asymmetry $Y$ & & & -0.6811 & 0.5872 & -0.574 & \\
\hline Stride Length & & 0.5403 & & & & \\
\hline Stride Time & & 0.7978 & & 0.7286 & -0.7065 & -0.6657 \\
\hline Left Step Time & & 0.7601 & & 0.6612 & -0.6463 & -0.6638 \\
\hline Right Step Time & & 0.8109 & & 0.7693 & -0.7414 & -0.6509 \\
\hline Peak to Peak Z & & -0.7494 & & & & \\
\hline Bounce & 0.5861 & -0.9139 & & & & \\
\hline Entropy Y & & 0.5304 & & & & \\
\hline Entropy Z & 0.6521 & & & & & \\
\hline
\end{tabular}

PEARSON CORRELATION "R" VALUES FOR RESIDENT 1 (NON-VEST). ALL CORRELATIONS SHOWN ARE STRONG $(|R|>0.40)$ OR VERY STRONG $(|R|>$ 0.70) AND ALL NON-ZERO CORRELATIONS ARE SIGNIFICANT (P<0.05). EXISTING FALL RISK ASSESSMENTS ARE SHOWN IN EACH COLUMN, AND METRICS DEVELOPED IN THIS STUDY ARE SHOWN IN ROWS. METRICS WITHOUT ANY CORRELATION ARE OMITTED. 
do not show correlation, all metrics except for trunk sway, show correlation to an established FRA in at least 3 residents. Additionally, all FRA's show correlation to at least 8 metrics. This shows that while the optimal choice of metrics varies from resident to resident, likely based upon their particular medical condition, there are a substantial set of metrics to choose from.

Since the relationship between FRA and the proposed metrics has some dependency on the resident's condition, we aggregated the data for all residents in the hopes of finding some overall correlation between the FRA results and metrics. The results are shown in Table 3-4. With all

Table 3-3: Subjects With Strong Correlation Between Proposed Metrics and fras

\begin{tabular}{cccccccccc} 
& Side & HGS & $\begin{array}{c}\text { Funct. } \\
\text { Reach }\end{array}$ & BBS & TUG & SPPB & SLS & $\begin{array}{c}\text { M } \\
\text { AX }\end{array}$ & TOT. \\
\hline Speed & 2 & 1 & 0 & 2 & 3 & 1 & 1 & 3 & 10 \\
Ten Foot Walk & 2 & 2 & 0 & 2 & 2 & 1 & 1 & 2 & 10 \\
Walk Efficiency & 1 & 1 & 1 & 0 & 1 & 0 & 1 & 2 & 5 \\
Asymmetry X & 0 & 1 & 0 & 2 & 1 & 1 & 0 & 2 & 5 \\
Asymmetry Y & 0 & 0 & 0 & 2 & 2 & 4 & 0 & 4 & 8 \\
Asymmetry Z & 0 & 1 & 1 & 0 & 0 & 1 & 0 & 1 & 3 \\
Stride Length & 1 & 2 & 0 & 0 & 0 & 0 & 0 & 2 & 3 \\
Stride Time & 1 & 3 & 0 & 1 & 2 & 1 & 1 & 3 & 9 \\
Left Step Time & 1 & 3 & 0 & 1 & 2 & 1 & 3 & 3 & 11 \\
Right Step Time & 1 & 3 & 0 & 1 & 2 & 1 & 1 & 3 & 9 \\
P2P X & 1 & 3 & 2 & 2 & 0 & 1 & 0 & 3 & 10 \\
P2PY & 0 & 2 & 1 & 1 & 1 & 2 & 0 & 2 & 7 \\
P2P Z & 0 & 2 & 1 & 0 & 1 & 0 & 2 & 2 & 6 \\
Step Ratio & 0 & 1 & 0 & 0 & 0 & 0 & 2 & 2 & 3 \\
Bounce & 3 & 2 & 2 & 0 & 1 & 0 & 2 & 3 & 10 \\
Sway & 1 & 1 & 0 & 0 & 0 & 0 & 0 & 1 & 2 \\
Entropy X & 0 & 2 & 1 & 1 & 0 & 1 & 0 & 2 & 5 \\
Entropy Y & 0 & 2 & 0 & 2 & 0 & 2 & 0 & 2 & 6 \\
Entropy Z & 1 & 1 & 1 & 1 & 1 & 0 & 0 & 1 & 5 \\
Entropy XY & 1 & 2 & 0 & 1 & 1 & 1 & 0 & 2 & 6 \\
Totals & 16 & 35 & 10 & 19 & 20 & 18 & 14 & 45 & 133 \\
& & & & & & & & &
\end{tabular}

This table shows the number of residents showing strong correlation ( $|r|>0.4)$ between the metric (left side) and established Fall Risk Analysis (top row). In this table, the total number of residents was 15. The MAX column shows the maximum number of residents with strong correlation for that metric. The TOTAL column shows the total number of strong correlations for that particular metric. All correlations shown in this table are statistically significant ( $p<=0.05$ ). All subjects for whom data is shown in this table did not participate in the weighted vest portion of the study. 
patient data aggregated, there is still strong correlation between the Berg Balance Score and the Walk Asymmetry in the Y direction (side-to-side), Habitual Gait Speed and Peak-to-Peak motion in the $Y$ direction and Trunk Sway. Additionally, seven metric-FRA pairs are moderately correlated $(r>0.3)$ with the 26 showing minor or no correlation.

\section{2 - Weighted Vest Therapy Results}

Table 3-4: Correlations Between Proposed Metrics and FRAs With AGGREGATED DATA

With a set of validated metrics that

\begin{tabular}{|c|c|c|c|c|}
\hline & Reach & BBS & TUG & SPPB \\
\hline Speed & & 0.206 & & \\
\hline Ten Foot Walk & & -0.269 & & -0.246 \\
\hline Walk Efficiency & & 0.222 & & 0.208 \\
\hline Asymmetry $\mathrm{X}$ & 0.258 & 0.258 & & 0.253 \\
\hline Asymmetry $Y$ & & 0.412 & -0.234 & 0.336 \\
\hline Asymmetry Z & & & 0.206 & \\
\hline Stride Length & & 0.207 & & \\
\hline Stride Time & & -0.258 & & -0.255 \\
\hline Left Step Time & & -0.280 & & -0.274 \\
\hline Right Step Time & & -0.235 & & -0.234 \\
\hline Peak to Peak X & & -0.213 & & -0.261 \\
\hline Peak to Peak Y & & & & -0.213 \\
\hline Entropy XY & & 0.252 & & 0.201 \\
\hline
\end{tabular}
each resident, histograms similar to fig. 3-

3 are generated for each metric and the

Pearson correlations between the different FRA instruments and various proposed metrics. Correlations are performed by number of "Minimum Detectable Changes aggregating metric and FRA pairs for all subjects. Missing cells indicate negligible correlation $(r<0.20)$ or statistically insignificant correlation ( $p>0.05)$. Metrics and FRA instruments without any (MDC's)" are computed. As can be seen correlations are not shown.

from the Table 3-5, all of the optimal window sizes for "vest on" events are equal to or shorter than for the corresponding "vest off" events. This suggests that, while variable for each subject, the body's response to putting the vest on is as quick as or quicker than taking the vest off. It also shows that the body's response to the vest is not immediate but requires some time to have an effect. 
The results for the subjects participating

Table 3-5: Window Size fOR Weighted Vest AnAlysis

in the vest study are shown in tabular form

Subject Vest On Window $\quad$ Vest Off Window

in Table 3-6 and graphically in fig. 3-5.

V1

2 hours, 45 minutes.

4 hours

Impact is reported in terms of number of

$\sqrt{2}$

3 hours, 30 minutes.

6 hours, 30 minutes.

V3 4 hours

4 hours

MDCs; the value of one MDC is shown in

This table shows the optimal analysis window size for each subject and each event. Interestingly, the vest on window is shorter or the

parentheses. These results are referenced same length as the vest off window suggesting a potentially faster response to donning the vest. Also, all of these windows are on the order of a few hours showing, at least for these subjects, that the

throughout the next three sections detailing effects of the vest are not immediate, but take some time to show an effect.

the results of each subject.

\subsection{1 - Subject \#V1}

This subject's gait is described as "stiff and ridged" due to progressive supranuclear palsy. A stiff, rigid gait has characteristically less flexion in the knee joints. This results in a higher than normal amount of centroid movement during ambulation. When this subject donned the weighted vest, the result showed significant decreases in entropy in the $Y(-1.78), Z(-1.43)$, and $X Y(-2.49)$ directions. Additionally, the peak-to-peak values also decreased for the $Y$ and $Z$ directions (-1.28 and -1.34 respectively).

When the vest is taken off, entropy in the $\mathrm{Y}$ direction returns to close to the original value. In the $\mathrm{XY}$ direction, there is a potentially similar increase in entropy though this value is only 0.95 MDC. In the $Z$ direction, entropy further decreased (-1.67) as well as for peak-to-peak in the $Z$ direction (-1.98). 
Table 3-6: Short Term Impact of the Weighted Vest Therapy on the Proposed Metrics

\begin{tabular}{|c|c|c|c|c|c|c|c|c|}
\hline \multirow[b]{3}{*}{$\begin{array}{l}\text { Speed } \\
\text { (ft./s) }\end{array}$} & \multicolumn{2}{|c|}{ V1 } & \multicolumn{2}{|c|}{ V2 } & \multicolumn{2}{|c|}{ V3 } & \multicolumn{2}{|c|}{ V3 (short) } \\
\hline & Vest On & Vest Off & Vest On & Vest Off & Vest On & Vest Off & Vest On & Vest Off \\
\hline & $\begin{array}{l}-1.48 \\
(0.0231)\end{array}$ & $\begin{array}{l}0.09 \\
(0.0362)\end{array}$ & $\begin{array}{l}-1.63 \\
(0.1014)\end{array}$ & $\begin{array}{l}2.12 \\
(0.0815)\end{array}$ & $\begin{array}{l}2.06 \\
(0.0137)\end{array}$ & $\begin{array}{l}-0.39 \\
(0.0138)\end{array}$ & $\begin{array}{l}-0.51 \\
(0.0335)\end{array}$ & $\begin{array}{l}-2.00 \\
(0.0335)\end{array}$ \\
\hline $\begin{array}{l}\text { Ten Foot Walk } \\
\text { (s) }\end{array}$ & $\begin{array}{l}2.14 \\
(0.0541)\end{array}$ & $\begin{array}{l}-0.81 \\
(0.0863)\end{array}$ & $\begin{array}{l}1.21 \\
(0.1630)\end{array}$ & $\begin{array}{l}-1.56 \\
(0.1404)\end{array}$ & $\begin{array}{l}-1.29 \\
(0.0759)\end{array}$ & $\begin{array}{l}1.05 \\
(0.0759)\end{array}$ & $\begin{array}{l}0.15 \\
(0.1731)\end{array}$ & $\begin{array}{l}1.36 \\
(0.1729)\end{array}$ \\
\hline Walk Efficiency & $\begin{array}{l}-2.89 \\
(0.0019)\end{array}$ & $\begin{array}{l}3.98 \\
(0.0016)\end{array}$ & $\begin{array}{l}3.81 \\
(0.0011)\end{array}$ & $\begin{array}{l}-6.14 \\
(0.0009)\end{array}$ & $\begin{array}{l}-1.36 \\
(0.0021)\end{array}$ & $\begin{array}{l}-0.05 \\
(0.0020)\end{array}$ & $\begin{array}{l}-0.76 \\
(0.0017)\end{array}$ & $\begin{array}{l}-2.34 \\
(0.0017)\end{array}$ \\
\hline asymmetry $\mathrm{X}$ & $\begin{array}{l}-0.64 \\
(0.0009)\end{array}$ & $\begin{array}{l}5.72 \\
(0.0002)\end{array}$ & $\begin{array}{l}-0.64 \\
(0.0035)\end{array}$ & $\begin{array}{l}-0.87 \\
(0.0036)\end{array}$ & $\begin{array}{l}1.95 \\
(0.0002)\end{array}$ & $\begin{array}{l}2.94 \\
(0.0002)\end{array}$ & $\begin{array}{l}1.10 \\
(0.0006)\end{array}$ & $\begin{array}{l}-1.32 \\
(0.0007)\end{array}$ \\
\hline asymmetry $Y$ & $\begin{array}{l}-0.83 \\
(0.0017)\end{array}$ & $\begin{array}{l}-1.64 \\
(0.0161)\end{array}$ & $\begin{array}{l}-2.06 \\
(0.0089)\end{array}$ & $\begin{array}{l}-0.78 \\
(0.0076)\end{array}$ & $\begin{array}{l}-1.89 \\
(0.0235)\end{array}$ & $\begin{array}{l}0.14 \\
(0.0235)\end{array}$ & $\begin{array}{l}-16.22 \\
(0.0048)\end{array}$ & $\begin{array}{l}1.12 \\
(0.0048)\end{array}$ \\
\hline asymmetry Z & $\begin{array}{l}-3.32 \\
(0.0008)\end{array}$ & $\begin{array}{l}-0.12 \\
(0.0017)\end{array}$ & $\begin{array}{l}-2.65 \\
(0.0054)\end{array}$ & $\begin{array}{l}12.62 \\
(0.0014)\end{array}$ & $\begin{array}{l}-1.42 \\
(0.0058)\end{array}$ & $\begin{array}{l}0.02 \\
(0.0050)\end{array}$ & $\begin{array}{l}-5.21 \\
(0.0018)\end{array}$ & $\begin{array}{l}1.16 \\
(0.0018)\end{array}$ \\
\hline $\begin{array}{l}\text { Stride Length } \\
\text { (ft) }\end{array}$ & $\begin{array}{l}-3.72 \\
(0.0224)\end{array}$ & $\begin{array}{l}3.40 \\
(0.0288)\end{array}$ & $\begin{array}{l}-0.55 \\
(0.1057)\end{array}$ & $\begin{array}{l}1.54 \\
(0.1039)\end{array}$ & $\begin{array}{l}1.31 \\
(0.0157)\end{array}$ & $\begin{array}{l}-1.33 \\
(0.0157)\end{array}$ & $\begin{array}{l}-0.33 \\
(0.1068)\end{array}$ & $\begin{array}{l}-0.92 \\
(0.1068)\end{array}$ \\
\hline $\begin{array}{l}\text { Stride Time } \\
\text { (s) }\end{array}$ & $\begin{array}{l}-6.25 \\
(0.0013)\end{array}$ & $\begin{array}{l}3.18 \\
(0.0073)\end{array}$ & $\begin{array}{l}15.53 \\
(0.0038)\end{array}$ & $\begin{array}{l}-4.66 \\
(0.0021)\end{array}$ & $\begin{array}{l}-2.19 \\
(0.0067)\end{array}$ & $\begin{array}{l}0.84 \\
(0.0067)\end{array}$ & $\begin{array}{l}1.18 \\
(0.0141)\end{array}$ & $\begin{array}{l}0.05 \\
(0.0145)\end{array}$ \\
\hline $\begin{array}{l}\text { Left Step Time } \\
\text { (s) }\end{array}$ & $\begin{array}{l}-1.86 \\
(0.0031)\end{array}$ & $\begin{array}{l}2.75 \\
(0.0085)\end{array}$ & $\begin{array}{l}74.92 \\
(0.0007)\end{array}$ & $\begin{array}{l}-0.37 \\
(0.0055)\end{array}$ & $\begin{array}{l}-0.87 \\
(0.0123)\end{array}$ & $\begin{array}{l}0.36 \\
(0.0123)\end{array}$ & $\begin{array}{l}2.55 \\
(0.0114)\end{array}$ & $\begin{array}{l}0.36 \\
(0.0112)\end{array}$ \\
\hline $\begin{array}{l}\text { Right Step Time } \\
\text { (s) }\end{array}$ & $\begin{array}{l}-19.69 \\
(0.0006)\end{array}$ & $\begin{array}{l}3.68 \\
(0.0062)\end{array}$ & $\begin{array}{l}9.14 \\
(0.0069)\end{array}$ & $\begin{array}{l}-13.71 \\
(0.0013)\end{array}$ & $\begin{array}{l}-17.13 \\
(0.0011)\end{array}$ & $\begin{array}{l}6.45 \\
(0.0011)\end{array}$ & $\begin{array}{l}0.26 \\
(0.0170)\end{array}$ & $\begin{array}{l}-0.37 \\
(0.0169)\end{array}$ \\
\hline $\begin{array}{l}\text { Peak to Peak X } \\
\text { (in) }\end{array}$ & $\begin{array}{l}-0.34 \\
(0.0238)\end{array}$ & $\begin{array}{l}-9.42 \\
(0.0268)\end{array}$ & $\begin{array}{l}-4.21 \\
(0.0546)\end{array}$ & $\begin{array}{l}1.52 \\
(0.0543)\end{array}$ & $\begin{array}{l}1.66 \\
(0.0605)\end{array}$ & $\begin{array}{l}-1.04 \\
(0.0605)\end{array}$ & $\begin{array}{l}-0.70 \\
(0.0995)\end{array}$ & $\begin{array}{l}-0.92 \\
(0.0995)\end{array}$ \\
\hline $\begin{array}{l}\text { Peak to Peak Y } \\
\text { (in) }\end{array}$ & $\begin{array}{l}-1.28 \\
(0.1755)\end{array}$ & $\begin{array}{l}0.21 \\
(0.2362)\end{array}$ & $\begin{array}{l}1.14 \\
(0.3875)\end{array}$ & $\begin{array}{l}1.12 \\
(0.3537)\end{array}$ & $\begin{array}{l}-1.39 \\
(0.2018)\end{array}$ & $\begin{array}{l}0.71 \\
(0.2018)\end{array}$ & $\begin{array}{l}-2.39 \\
(0.0624)\end{array}$ & $\begin{array}{l}-2.23 \\
(0.0624)\end{array}$ \\
\hline $\begin{array}{l}\text { Peak to Peak Z } \\
\text { (in) }\end{array}$ & $\begin{array}{l}-1.34 \\
(0.0705)\end{array}$ & $\begin{array}{l}-1.98 \\
(0.0720)\end{array}$ & $\begin{array}{l}-9.64 \\
(0.0571)\end{array}$ & $\begin{array}{l}1.66 \\
(0.0461)\end{array}$ & $\begin{array}{l}2.15 \\
(0.1150)\end{array}$ & $\begin{array}{l}-1.52 \\
(0.1151)\end{array}$ & $\begin{array}{l}-0.90 \\
(0.1205)\end{array}$ & $\begin{array}{l}1.01 \\
(0.1206)\end{array}$ \\
\hline Step Ratio & $\begin{array}{l}-1.61 \\
(0.0013)\end{array}$ & $\begin{array}{l}-39.97 \\
(0.0000)\end{array}$ & $\begin{array}{l}9.33 \\
(0.0030)\end{array}$ & $\begin{array}{l}-2.69 \\
(0.0058)\end{array}$ & $\begin{array}{l}2.03 \\
(0.0012)\end{array}$ & $\begin{array}{l}2.71 \\
(0.0012)\end{array}$ & $\begin{array}{l}0.75 \\
(0.0052)\end{array}$ & $\begin{array}{l}0.05 \\
(0.0052)\end{array}$ \\
\hline $\begin{array}{l}\text { Bounce } \\
\text { (in) }\end{array}$ & $\begin{array}{l}-7.32 \\
(0.0015)\end{array}$ & $\begin{array}{l}-5.96 \\
(0.0051)\end{array}$ & $\begin{array}{l}-2.66 \\
(0.0683)\end{array}$ & $\begin{array}{l}1.60 \\
(0.0697)\end{array}$ & $\begin{array}{l}4.28 \\
(0.0105)\end{array}$ & $\begin{array}{l}-6.13 \\
(0.0105)\end{array}$ & $\begin{array}{l}-0.10 \\
(0.0464)\end{array}$ & $\begin{array}{l}2.18 \\
(0.0467)\end{array}$ \\
\hline $\begin{array}{l}\text { Sway } \\
\text { (in) }\end{array}$ & $\begin{array}{l}-0.78 \\
(0.0561)\end{array}$ & $\begin{array}{l}0.45 \\
(0.0877)\end{array}$ & $\begin{array}{l}0.25 \\
(0.9900)\end{array}$ & $\begin{array}{l}1.13 \\
(0.7589)\end{array}$ & $\begin{array}{l}-1.98 \\
(0.1077)\end{array}$ & $\begin{array}{l}2.08 \\
(0.1076)\end{array}$ & $\begin{array}{l}-0.74 \\
(0.1045)\end{array}$ & $\begin{array}{l}-0.01 \\
(0.1017)\end{array}$ \\
\hline Entropy X & $\begin{array}{l}1.73 \\
(0.0250)\end{array}$ & $\begin{array}{l}-9.98 \\
(0.0212)\end{array}$ & $\begin{array}{l}-8.81 \\
(0.0461)\end{array}$ & $\begin{array}{l}3.82 \\
(0.0393)\end{array}$ & $\begin{array}{l}1.65 \\
(0.0933)\end{array}$ & $\begin{array}{l}-1.55 \\
(0.0933)\end{array}$ & $\begin{array}{l}0.01 \\
(0.1200)\end{array}$ & $\begin{array}{l}-0.85 \\
(0.1177)\end{array}$ \\
\hline Entropy $Y$ & $\begin{array}{l}-1.78 \\
(0.2316)\end{array}$ & $\begin{array}{l}1.46 \\
(0.2988)\end{array}$ & $\begin{array}{l}0.81 \\
(0.4408)\end{array}$ & $\begin{array}{l}1.18 \\
(0.4392)\end{array}$ & $\begin{array}{l}-0.49 \\
(0.4532)\end{array}$ & $\begin{array}{l}0.10 \\
(0.4528)\end{array}$ & $\begin{array}{l}0.63 \\
(0.2658)\end{array}$ & $\begin{array}{l}-1.53 \\
(0.2657)\end{array}$ \\
\hline Entropy Z & $\begin{array}{l}-1.43 \\
(0.1030)\end{array}$ & $\begin{array}{l}-1.67 \\
(0.1104)\end{array}$ & $\begin{array}{l}-6.21 \\
(0.0743)\end{array}$ & $\begin{array}{l}3.86 \\
(0.0192)\end{array}$ & $\begin{array}{l}1.80 \\
(0.1871)\end{array}$ & $\begin{array}{l}-1.21 \\
(0.1871)\end{array}$ & $\begin{array}{l}2.69 \\
(0.1351)\end{array}$ & $\begin{array}{l}1.22 \\
(0.1351)\end{array}$ \\
\hline Entropy $\mathrm{XY}$ & $\begin{array}{l}-2.49 \\
(0.1125)\end{array}$ & $\begin{array}{l}0.95 \\
(0.1963)\end{array}$ & $\begin{array}{l}-1.17 \\
(0.3704)\end{array}$ & $\begin{array}{l}1.77 \\
(0.3637)\end{array}$ & $\begin{array}{l}8.55 \\
(0.0239)\end{array}$ & $\begin{array}{l}-4.42 \\
(0.0239)\end{array}$ & $\begin{array}{l}0.08 \\
(0.3747)\end{array}$ & $\begin{array}{l}-1.06 \\
(0.3741)\end{array}$ \\
\hline
\end{tabular}

Impact on metrics (left column) of the vest therapy for three subjects. Values shown are the number of Minimum Detectable Change intervals represented by the change. The number in parenthesis below each value is the value of one MDC. The units for each metric, applicable to the MDC value, is shown under the name of the proposed metric. "Vest On" refers to the event when the vest is put on, and "Vest Off" refers to the event where the vest is taken off. Therefore, the "Vest On" refers to the change in the proposed metric from when the vest was off to when it was on. * Positive asymmetry values represent a positive change in the absolute value of the metric - towards a more asymmetrical walk. Negative values correspond to a change towards a more symmetrical walk. 


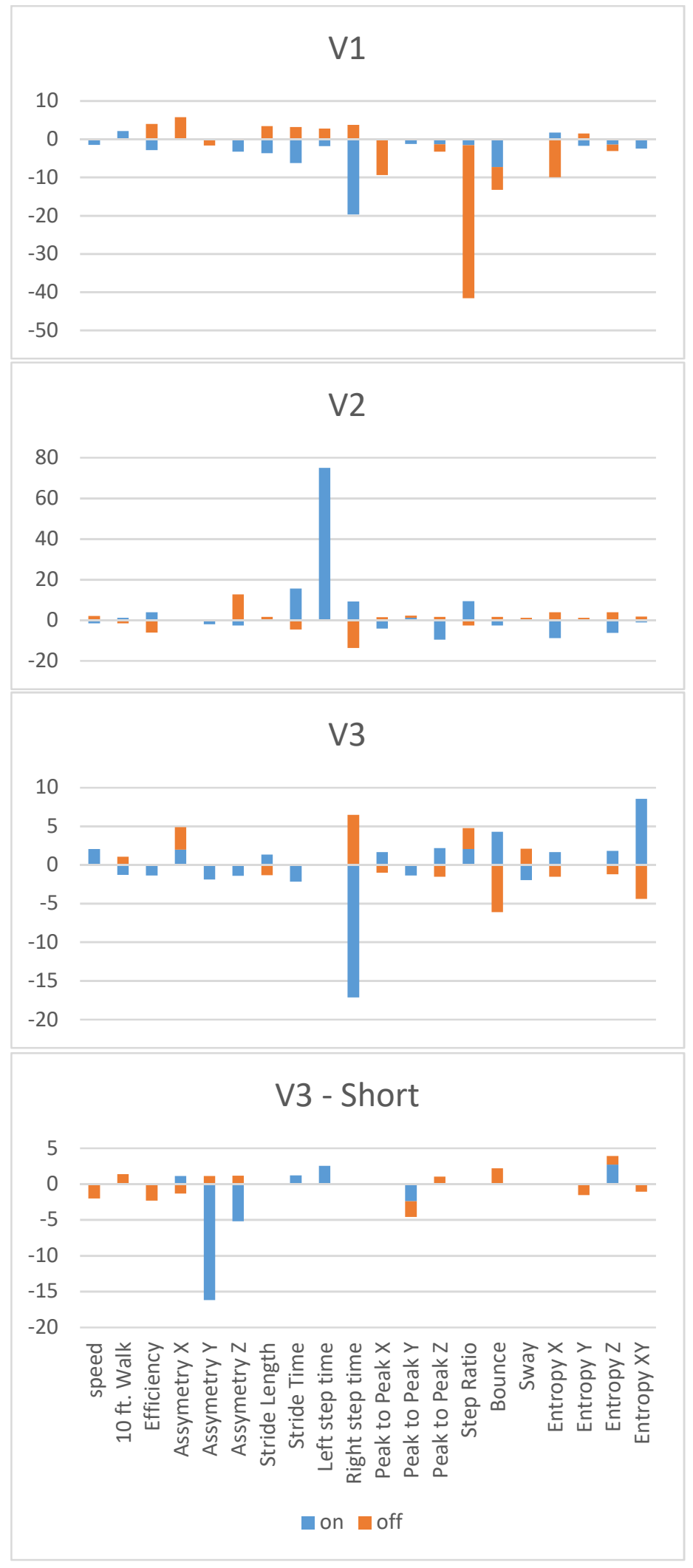

Figure 3-5: Graphical representation of the data in Table VI. These figures show the number of MDC's that the metric has changed when the vest is both taken off (red bars) and put on (blue bars). Metrics for which the change is less than one MDC are recorded as zero on these graphs. 
The gait parameters, however, showed a more consistent behavior when used with the vest. When the vest was donned, the subject showed an increase in 10 foot walk time (2.14), a decrease in both step times (-1.86, and -19.69 for left and right respectively), and -6.25 stride time, stride length (-3.72) and a decrease in walk efficiency (-2.89). Walking speed also decreased by -1.48 . All totaled this seems to suggest shorter and more frequent steps. When the vest was removed, all parameters had a significant change in the opposite direction except for both 10 foot walk times

\subsection{2 - Subject \#V2}

Subject number two is characterized by a looser gait. In addition to the clinical assessment, this is confirmed mostly by a decrease in entropy in the $X, Z$, and combined $X Y$ directions $(-8.81,-6.21$, and -1.17 respectively) when the vest is put on. There is also a reduction in asymmetry in the $Y$ and $Z$ directions (-2.06 and -2.65). Lastly, peak-to-peak motion in the $X$ and $Z$ directions also decreases when the vest is put on (-4.21 and -9.64 respectively). When the vest is removed, the above three entropy values also show significant increase $(3.82,3.86$, and 1.77 respectively). Asymmetry in the $Y$ direction does not show any significant change, while Asymmetry in the $Z$ direction does show an increase of 12.62 MDC's. Both peak-to-peak measurements ( $\mathrm{X}$ and Z directions) show significant increases (1.52 and 1.66) suggesting a partial return towards pre-vest values.

Gait metrics tell a more compelling story. When the vest is put on, significant increases are seen in the 10 foot walk time (1.21), both step times (right (9.14), left (74.92)) and stride time (15.53). When the vest was removed, all of these metrics (except for left step time) showed a significant change back towards the pre-vest value (10 foot Walk: -1.56 ; step times: $r=-13.71, \mathrm{l}=-$ 0.37 (not significant), stride time=-4.66). Stride length also showed a significant increase (1.54). 
Speed shows a decrease $(-1.63$ when

donned; 2.12 when doffed) which is

consistent with an increase and

decrease in time needed to walk 10

feet. Walk efficiency increased when

the vest was donned (3.81 MDC) and

subsequently dropped back beyond

initial value when the vest was

removed.

\subsection{3 - Subject \#3}

Subject \#3 also has a looser gait.

When the vest was put on, entropy

increased in the $X Y$ and $Z$ directions
Table 3-7: Metrics Sensitivity

\begin{tabular}{lll} 
& On & Ott \\
\hline Speed & 1.72 & 0.87 \\
Ten Foot Walk & 1.55 & 1.14 \\
Walk Efficiency & 2.69 & 3.39 \\
Asymmetry X & 1.08 & 3.18 \\
Asymmetry Y & 1.59 & 0.85 \\
Asymmetry Z & 2.46 & 4.25 \\
Stride Length & 1.86 & 2.09 \\
Stride Time & 7.99 & 2.89 \\
Peak to Peak X & 2.07 & 3.99 \\
Peak to Peak Y & 1.27 & 0.68 \\
Peak to Peak Z & 4.38 & 1.72 \\
Left Step Time & 25.88 & 1.16 \\
Right Step Time & 15.32 & 7.95 \\
Step Ratio & 4.32 & 15.12 \\
Bounce & 4.75 & 4.56 \\
Sway & 1.00 & 1.22 \\
Entropy X & 4.06 & 5.12 \\
Entropy Y & 1.03 & 0.91 \\
Entropy Z & 3.15 & 2.25 \\
Entropy XY & 4.07 & 2.38 \\
\end{tabular}

Sensitivity of each Metric for all three subjects. This table shows the average number of $M D C^{\prime}$ 's registered by each metric for all residents. From the results obtained, the most sensitive metrics are the temporal metrics, particularly left and right step time, step ratio, average step time. Of the more sensitive metrics, most are more sensitive to the vest on event than the vest off event.

(8.55 and 1.80 respectively). Peak-to-Peak in the $Y$ direction decreased (-1.39) while it increased in the $Z$ direction (2.15). Asymmetry decreased in the $Y$ and $Z$ directions $(-1.89$ and -1.42 respectively) when the vest was donned. Removing the vest causes a significant change in the opposite direction in entropy $X Y$ and $Z$ directions (-4.42 and -1.21) peak-to-peak $Z(-1.52)$ There were no significant changes in Asymmetry $\mathrm{Y}$ or $\mathrm{Z}$ when the vest was removed.

The temporal parameters showed negative changes in 10 foot walk time, (-1.29), and right step time (-17.13). Positive changes were seen in stride length (1.31). When the vest was taken off, positive changes are seen in both the 10 foot walk (1.05) and right step time (6.45), while a negative change was seen in stride length (-1.33). This subject showed an increase in speed as the vest was put on and a potential, but not significant, drop in speed when it was removed. 
For this subject, we also took a brief look at the very short term effect of the vest by looking at only the half hour before and after the vest is donned and doffed. While most of the metrics did not show any significant change over that short time period, there were significant changes in all three asymmetry metrics. When the vest was put on, asymmetry in the $\mathrm{Y}$ and $\mathrm{Z}$ directions both decreased (-16.22 and -5.21 respectively) and increased in the $X$ direction (1.10). All three of these metrics changed significantly in the opposite direction $(1.12,1.16$, and -1.32 respectively) when the vest was taken off. This result confirms what was seen during development - different metrics change over different periods of time, and that by choosing different time windows, different conditions can be studied.

\section{3 - Sensitivity of Metrics}

Lastly, the sensitivity of each metric was gauged by averaging the absolute value of the MDC changes for all subjects. The results of this analysis are shown in Table 3-7. From the results shown, for these subjects, the temporal metrics appear to be the most sensitive. Entropy in the $\mathrm{X}$ direction and bounce also appear to be more sensitive while peak-to-peak in the $\mathrm{Y}$ direction and entropy in the $\mathrm{Y}$ direction appear to be the least.

\section{5 - Discussion}

\section{1 - Validation}

The proposed algorithm scored within $2 \%$ of the Vicon ${ }^{\circledast}$ generated results with 13 different subjects with multiple walks. Next, the algorithm was tested against a validated reference algorithm using data obtained from in home subjects captured in the subject's home. Data from seven subjects were selected and all had less than $0.26 \%$ error in the mean value of stride time. Stride length and average speed, while slightly less accurate, were still accurate within $7 \%$ and $3 \%$ respectively. 


\section{2 - Relationship to FRA}

Comparisons to existing FRA's were done to assess the potential of comparing these metrics to clinical fall risk assessments. Each individual subject had a different set of correlation values between the set of metrics and FRA results. While you would expect, and we have seen in Tables 3-2 and 3-3, some relationship between step time and the TUG test, the TUG test includes a requirement for the subject to execute a 180 degree turn. The ability to execute a proper 180 degree turn depends on the subject's ability to balance. This would suggest some relationship between the TUG and balance measurements. As also seen in Table 3-2, there is a strong negative correlation between the BBS score and asymmetry as well as a positive correlation between TUG and asymmetry. This confirms, at least in this subject, that the BBS and TUG measure common components of gait.

The relationship between FRA's and metrics are, however, different between different subjects. The results in Table 3-3 show that, while not every metric relates to each FRA in the patient population, there is enough overlap between these to show several FRA's correlating to several proposed metrics. As each subject has a different set of symptoms that will impact balance and/or gait, you would expect each subject to have a different set of correlations. Table 3-3 shows the smallest set of correlations between the metrics and the functional reach FRA. The largest set of correlations is shown for Habitual Gait Speed. Similarly, the metrics with the most overlap with FRA's are Step times, 10 foot walk, speed and peak-to-peak in the $Y$ direction. 


\section{3 - Use with the Weighted Vest}

Lastly, this full set of metrics was used to study the weighted vest therapy on 3 different subjects. Overall, the changes in these metrics are consistent with expected changes based upon the subject's physical condition.

\subsection{1 - Subject V1}

For the rigid gait of subject $\# \mathrm{~V} 1$, the weighted vest caused a decrease in lateral entropy followed by an increase when the vest was removed. This is backed up by a decrease in peak-to-peak movement in the $Y$ direction. Looking at all of the changed temporal metrics together, a picture of the effect on the subject starts to appear. The 10 foot walk time metric increased and overall speed decreased. This is, at first glance, counter-intuitive as you may expect an increase in walk speed as the subject's ability to ambulate "improves". Factoring in stride length, stride time, right step time, and left step time, paints a picture of a subject taking shorter, quicker steps. Lastly, the subject's walk efficiency is shown to drop when the vest is donned and increase when the vest is removed.

With the vest removed, entropy in the $Y$ direction, stride length, all both step times (left, right), and stride time changed back towards their values before the vest was put on. Speed, 10 foot walk, and peak-to-peak in the $Y$ direction had very little change when the vest was removed suggesting a possible residual effect of the vest therapy after the vest is removed.

\subsection{2 - Subject V2}

Subject V2 also shows an increase in the 10 foot walk times and a reduction in speed when the vest is donned. Additionally, right and left step times and stride time increase with a slight, but not statistically significant, decrease in stride length. Looked at together, this indicates slower, 
more deliberate steps of the similar length. The subject's walk becomes more efficient, even with a small but significant increase in peak-to-peak motion in the $Y$ direction suggesting that additional "rocking" or swaying during the walk might improve the overall directness of the walking path. A drop in entropy in the $X$ and $X Y$ directions suggest a smoother walk as a result of the therapy as does the decrease in peak-to-peak motion in the $\mathrm{X}$ direction.

For virtually all of the metrics discussed, removing the vest causes a change in the opposite direction except for left step time which, while showing a decrease, was not a significant change. Removing the vest also caused a significant increase in stride length. This suggests a smaller residual effect of the vest on this particular subject.

\subsection{3 - Subject V3}

The addition of the $30 \mathrm{~min}$. window analysis showed a difference in the progression of the vest therapy on different parameters. This run suggests that, for this subject, all three asymmetry metrics change quickly when the vest is donned and doffed. Few of the remaining metrics had any significant change when the vest was donned while more had changed significantly when the vest was removed. Using a window size of 3 hours (more in line with the other two subjects), a much larger group of metrics show significant changes. The three asymmetry metrics still show changes when the vest is donned, but no longer show a significant change when the vest is doffed.The longer term window showed a decrease in the 10 foot walk time, and an increase in speed. It also showed an increase in stride length and a decrease in stride time, although the

decrease in left step time was not significant. Taken together, this shows the subject is simply walking faster with longer and quicker steps. Asymmetry in the $\mathrm{Y}$ and $\mathrm{Z}$ directions decrease (though there is no significant return to previous values with the longer window). 


\section{4 - Limitations}

Two limitations with using centroid based metrics involve multiple subjects and large obstructions. If two or more people are routinely present in the monitored area, it is necessary to separate them before analysis can be done. In this study, the Gaussian Mixture Model clustering algorithm was able to adequately separate people with significantly different height and/or walking speeds. Of the four total subjects who participated in the strategically weighted vest study, the data from one was omitted from this study. The subject had a similar height and walking speed as another person who was frequently in the apartment. The clustering algorithm was unable to separate these two people's walks adequately. An improved clustering algorithm combined with a different feature set will be tried in the hopes of better separating the walks by each person.

A second limitation is large obstructions. One of the benefits of using the centroid to compute gait parameters is the ability to monitor even if the subject's feet are obstructed. A typically cluttered living area may have foot stools, ottomans, or coffee tables which may, from time to time, obstruct the subject's feet. Using centroids to compute gait parameters reduces the impact of these obstructions on generating reliable metrics. Larger obstructions, however, may negatively impact the computation of these metrics. If enough of the subject is obstructed, the size of the point cloud used to compute the centroids may be small enough to cause distortions to the calculated metrics. This can be addressed by applying a minimum size threshold to the point cloud and rejecting all walks for which the point cloud is too small.

\section{6 - Conclusions}

A toolbox of metrics and analysis approaches are presented that can be used to study intervention effects on gait. The large assortment of metrics allows a clinician to choose which 
metric(s) to use based upon what is being studied. For example, if a TUG test shows a change, these metrics can be used to determine if it was due to a change in stride time, stride length, or entropy (suggesting balance). Comparing these metrics against established FRAs shows strong to very strong correlation. These correlations, however, appear to be dependent upon the subject's individual physical condition and, potentially, disease state. The relationships are consistent with the components of both the FRA instruments and the physical and physiological components of the metrics. When aggregated across all subjects, three metric/FRA pairs showed strong correlation, 4 show moderate correlation, and 25 show weak correlation.

Results also show that response to the strategically weighted vest therapy is not immediate but, at least for these subjects, requires time on the order of a few hours to reach full effect. These results also show that the body responds as quickly or more quickly to the vest being donned than when the vest is removed. These results also show that the algorithm can be used to determine the effects on each metric over specific periods following the events. In other words, it can be used to answer the question "Which metrics show the most change within 1 hour of the vest being taken off".

Finally, the metrics previously presented in [9] are now presented in the larger context of temporal metrics and overall subject gait. The results of three participants in the weighted vest study, each with unique physical conditions, demonstrate the ability of these metrics to identify changes in the subject's ambulation as a result of the vest therapy. The results also show that the subject shows significant changes in some metrics soon after donning the vest, while other metrics are slower to respond. Future work will include study into the long term effects, measured over days and weeks, of the vest therapy as well as shorter term effects of the vest on these aspects of the subject's gait. It will also study, more in depth, the relationship between 
FRA's and these metrics. Results also suggest that the temporal metrics are the most sensitive to changes following wearing or removal of the vest.

\section{Acknowledgements}

The research is funded by the NSF under grant CNS-0931607. 


\section{Appendix I}

What follows is a list of all of the variables used throughout this paper and their meanings.

Unless qualified as pertaining to a particular computation in the table, variables names and their usage are consistent throughout all formulae.

\begin{tabular}{|c|c|}
\hline$x, y, z$ & $\begin{array}{l}\text { Actual centroid location in the } \mathrm{X} \text { (along the direction of travel), } \mathrm{Y} \text { (perpendicular to } \\
\mathrm{X} \text { in the horizontal plane), and } \mathrm{Z} \text { (up / down) directions. }\end{array}$ \\
\hline$x^{\prime}, y^{\prime}, z^{\prime}$ & $\begin{array}{l}\text { Expected location of the centroid in the walking path. To compute this, a best fit } \\
\text { line is determined over }+/-0.5 \text { seconds centered on the current point. Using the line, } \\
\text { the centroid location at time }=t+1 \text { is determined. }\end{array}$ \\
\hline$\delta_{i}$ & $\begin{array}{l}\text { Error between the expected location and actual location of the centroid in the } i \\
\text { direction. }\end{array}$ \\
\hline$A_{i}$ & Asymmetry in the $i$ direction. \\
\hline$p 2 p_{i}$ & Peak to Peak travel in the $i$ directions. \\
\hline $\mathrm{s}$ & Walking speed. \\
\hline W & Ten Foot Walk Time \\
\hline$T$ & Duration of the walk \\
\hline $\mathrm{D}$ & Distance of the walk. \\
\hline E & Walk Efficiency \\
\hline$H_{D T E}^{\delta_{i}}$ & Deluca \& Termini computed entropy in the $i$ direction. \\
\hline$H_{P P E}^{\delta_{i}}$ & Pal computed entropy in the $i$ direction. \\
\hline$H_{\alpha Q E}^{\delta_{i}}$ & Pal and Bezdek computed entropy in the $i$ direction \\
\hline$\delta_{k j}$ & For entropy calculations, centroid position $j$ in the $k$ direction. \\
\hline K & For entropy calculations, a normalization constant chosen to be 1 in this paper. \\
\hline$\alpha$ & $\begin{array}{l}\text { For Pal-Bezdek entropy calculations, this controls the sensitivity of the entropy } \\
\text { value to changes in the input data. Set to } 0.75 \text { for this paper. }\end{array}$ \\
\hline$M$ & Vector containing the time indices of all the local minima in the $\delta_{z}$ signal. \\
\hline $\begin{array}{l}S T 1 \\
S T 2\end{array}$ & $\begin{array}{l}\text { Temporary values of step time during the calculation of left and right step time. } \\
\text { Based upon the direction of the first peak (either left or right) these are assigned to } \\
\text { be left and right step time. }\end{array}$ \\
\hline$B$ & Bounce measurement in gait. \\
\hline
\end{tabular}




\begin{tabular}{|l|l|}
\hline$S$ & Trunk sway measurement in gait. \\
\hline$M D C$ & $\begin{array}{l}\text { Minimum Detectable Change. MDC corresponds to the smallest change that can be } \\
\text { detected which cannot be attributed to random error. }\end{array}$ \\
\hline$M S E$ & Mean Squared Error estimated by the variance for the pre-event distribution. \\
\hline
\end{tabular}




\section{Chapter 4 PCA Assisted Clustering}

\section{Background}

Principle Component Analysis

In the study presented in chapter 3 as well as in [9], a Gaussian Mixture Model using height and speed as features [10] had been used. While effective on the data from most apartments, there were some apartments that could not be separated reliably, and their data was excluded. The PFCM algorithm, developed by Pal in 2005 [92], includes a fuzzy component that is useful for separating difficult feature sets. Simply using the PFCM algorithm yielded better results than had been obtained previously, but the clustering was still marginal at best and unreliable.

Principle Component Analysis (PCA) is a technique developed by Pearson in 1901 [117]. The technique is most often used for dimensional reduction of the feature space to better visualize or reduce the computational load during analysis. PCA transforms a muti-feature data set into a new coordinate system such that each component (column) of the matrix is orthogonal to all the other components.

PCA was used to study both the input data and clustering results in order to identify possible relationships in the features that remain invisible in the untransformed data. Observing that PCA analysis was very effective at visually separating clusters, clustering was attempted on PCA transformed data and yielded very good results. Although dimensional reduction was not the motivation for using PCA, the smallest set of features was chosen such that $95 \%$ of the total variance was captured.

The process utilized involves first normalizing each column such that the mean of each column is zero. Next, the covariance matrix of the data is computed, then the eigenvalues and eigenvectors are computed from the covariance matrix. Next, these eigenvectors are orthogonalized and normalized to become unit vectors. 
During the process of developing each component, the transformed matrix is ordered such that the first column captures the maximum variance. The second column will capture the maximum possible variance for what is left, and so on.

It is important to note that, for this study, the PCA transformed data is not fundamentally altered but simply projected along a different set of axes. Analogous to viewing a 3-D object from a different perspective to better visualize the object. This allows clustering to be performed on the PCA transformed data without any loss accuracy or corruption of results.

\section{iVAT}

The Improved Visual Assessment of Clustering Tendencies was developed in 2012 by Havens and Bezdek [118] as an improvement on the Visual Assessment of Clustering Tendencies approach. This visualization technique can be used to investigate the structure of a set of features and data points to identify possibly clusters of related data.

The technique creates a matrix of disparity values between all combinations of points in the data set. The disparity values are displayed in color or gray scale plot showing low disparity values in dark colors, and high values using light colors. The resulting image shows zero or more dark blocks indicating likely clusters in the original data. The number and relative sizes of the blocks suggest a number and relative size of different clusters in the data. To illustrate, Figure 51 shows an IVAT image of trunk sway for apartment 3037 (a two-resident apartment). The large block in the upper left corner and the smaller block in the lower right corner represent the walks for both residents. 


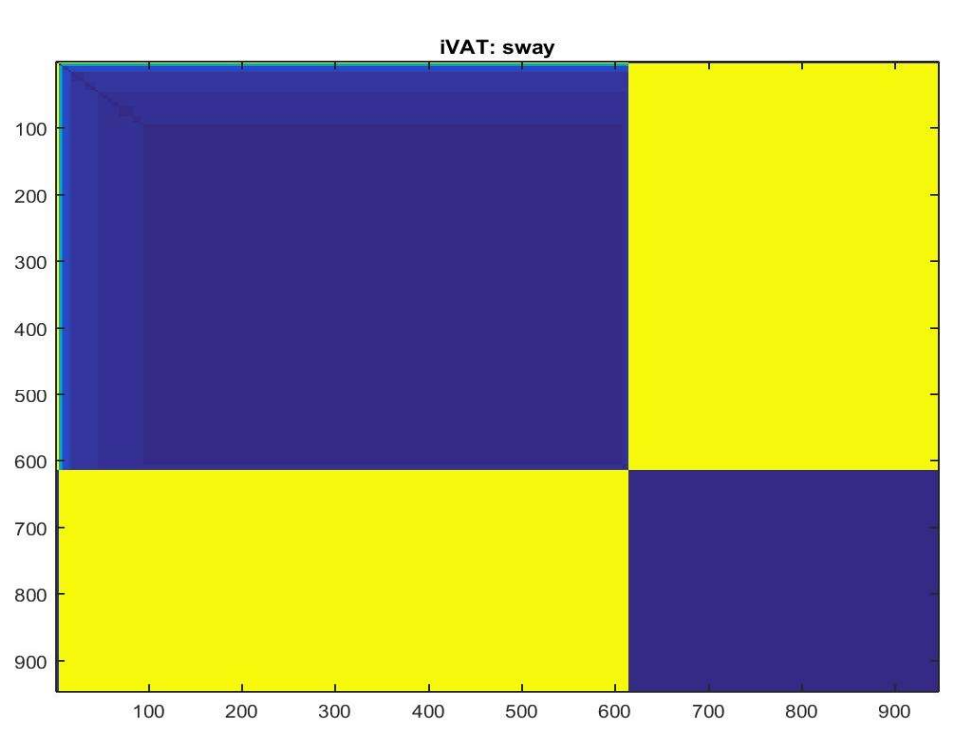

Figure 4-1 Example of an IVAT image for a two resident apartment. The two dark colored blocks represent potential clusters in the dataset.

While not used to actually cluster datasets, this image can be used to visualize the data in order to determine the number of clusters and qualitative sizes that are likely contained in the data.

\section{Possibilistic Fuzzy C-Means}

Possibilistic Fuzzy C-Means is an unsupervised clustering approach developed by Pal et al. in 2005 [119]. It solves weaknesses in both the Possibilistic C-Means and Fuzzy C-Means approaches by combining the two approaches with weighting factors which determine the relative contribution of the fuzzy and possibilistic aspects of this algorithm.

The fuzzy component of PFCM assigns membership values to each data point based upon the proximity of the point to the center of its cluster. The possibilistic component assigns typicality values showing the relative likelihood that the point is part of the cluster. As mentioned in [119], membership values are more useful when assigning crisp labels to data points while 
typicality values are more useful when estimating centroid locations and also help to reduce challenges with the undesirable side-effects associated with outliers.

The PFCM algorithm, as many other unsupervised learning algorithms, is an optimization problem with a cost function given here: [119]

$$
J_{m, \eta}(\boldsymbol{V})=\sum_{i=1}^{c} \sum_{k=1}^{n}\left(a u_{i k}^{m}+b t_{i k}^{\eta}\right) D_{i k A}^{2}
$$

In the equation, $a$ and $b$ determine the relative weights of the fuzzy and possibilistic behavior of the clustering. Setting $a=0$ will remove the fuzzy component and result in a behavior very similar to the Possibilistic C-Means algorithm. Similarly, setting $b$ to zero will cluster using an approach` very similar to the fuzzy C-Means algorithm. $D$ is the distance between two points, $u$ and $t$ are the fuzzy membership and typicality values which correspond in meaning and interpretation to the equivalent values in FCM and PCM.

\section{Feature Identification}

The initial attempt at clustering residents utilized a Gaussian Mixture Model (GMM) based upon subject height and walking speed to cluster the data collected from a single apartment. The GMM was initialized randomly and 50 models were computed for 2 clusters in a one-person apartment (one resident and others), and 3 clusters in a two person apartment (two residents and others). The Fowlkes Mallows score is computed for each model with the best score selected as the winner. The smallest cluster is discarded as it most likely contains visitors, guests, and other outliers. This method is similar to that used by Stone et al. [105]. This method worked for the simpler apartments and was published in the paper presented in chapter 3 . The method was

not, however, able to separate the more complicated apartments where residents are similarly sized and have similar walking speeds. An improved method was needed. 
Starting with the computed metrics for all walks in a particular apartment, the first step is to find a set of features that would help differentiate the residents. IVAT images were generated for each individual metric for each subject. These images, an example of which is shown in figure 4-2, can provide insight into the underlying clustering of each metric. The dark blue regions represent areas of low disparity between samples suggesting that those points belong in the same cluster.
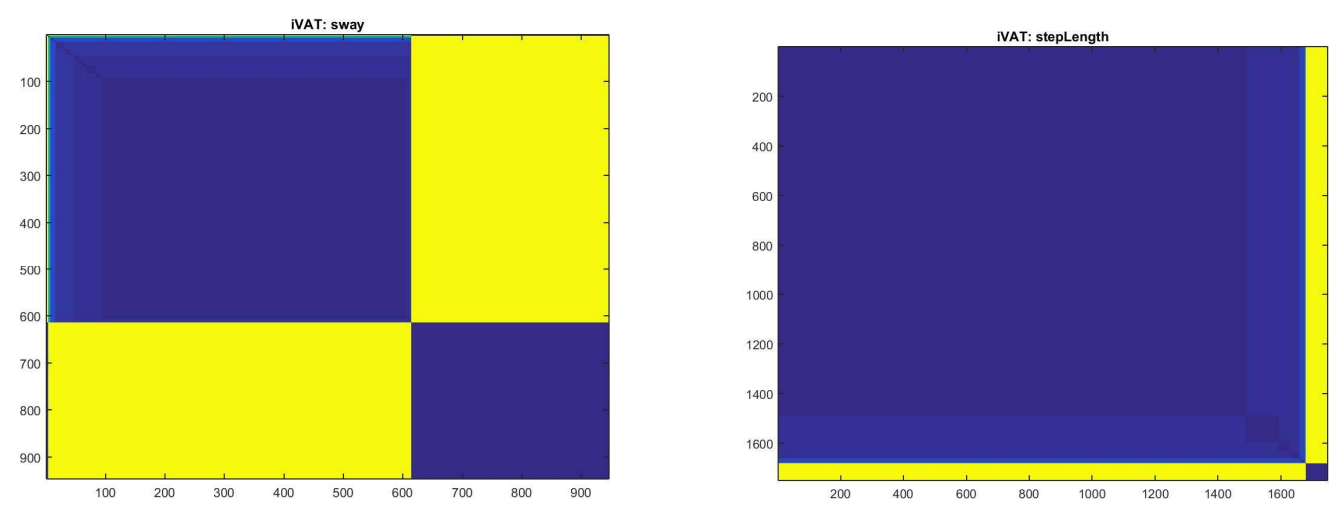

Figure 4-2: Two examples of an IVAT image for a single resident apartment (left) and a double resident apartment (right). The two blue squares (upper-left, lower-right) correspond to clusters of walks by each resident. In the top image, one resident walked roughly $60 \%$ of the time while in the bottom image, the small square represents less than $5 \%$ of the walks.

Once iVAT images were created for each individual metric, each image was classified on a scale of 1-4 based upon how much structure was shown in the image. iVAT images for each individual metric were generated for 3 different apartments and categorized. After examining each of the images, four "required" features and four "optional" features were selected. The set of features are shown in Table 4-1. The "required" features are used for every data set while the "optional" features are selected as described in the next section.

While identifying the features as described above, it was found that Table 4-1: Metrics Used for Resident Identification Clustering

\begin{tabular}{|l|l|}
\hline Required Metrics & Optional Metrics \\
\hline Step Time & Trunk Sway \\
\hline Total Height & Step Ratio \\
\hline Average Speed & Step Length \\
\hline Avg. Instantaneous Speed & XY Entropy \\
\hline
\end{tabular}


in some cases, reducing the number of metrics not only saved computational demand, but also improved the results. This makes sense as each person is unique and no two people present with a particular disease in the same way due to unique disease progression and a patient's unique history. What was needed was a way of selecting smallest set of features as long as the DaviesBouldin index does not increase. This was done, simply, by scanning all 16 combinations of the four optional features, combining them with the "required" features, and attempting to cluster the PCA transformed set of features from the first fourteen days of data.

This final set of metrics was then used to cluster the data. The data was divided into chunks representing two weeks. This was done to minimize the effects of long term changes in certain metrics which may result in misclassification. After the first clustering, the largest cluster was selected as the first resident. In a two-resident apartment, the second largest cluster was selected as the second resident. Remaining clusters are matched based upon the proximity of the centers of each cluster to the previous centers.

\section{Results}

The iVAT algorithm was implemented and validated with ad-hoc simulated data heavily based upon the test data used in [118]. An example of an attempt at clustering a full data set for

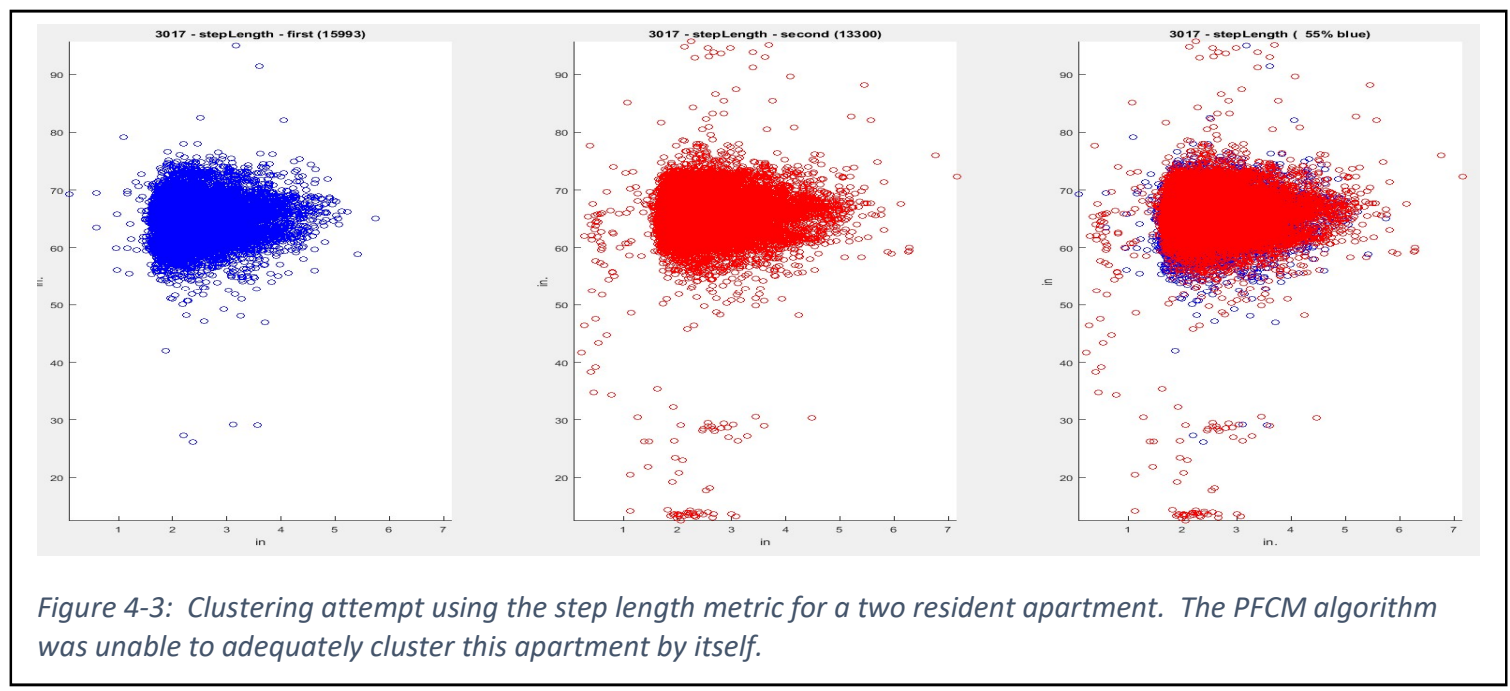


apartment 3017 using the PFCM algorithm is shown in figure 4-3. The next figure, figure 4-4, shows apartment 3047, also a two-resident apartment, clustered with PCA using 14 day chunks. The first pair of images shows the first two weeks. The second pair of images shows weeks 5 and 6 . The third shows weeks 7 and 8 , and the fourth shows weeks 9 and 10 .

Looking at these cluster sets, there is very little overlap between the clusters. The "red" resident in the right plots seems to be taller with a slightly longer step time, something not unexpected on a taller person. Looking at the second set of images (weeks 3 and 4) the results look very similar showing that the process is repeatable. The third set of images shows better separation based upon the individual's heights. Looking at the transformed images for the first two sets of images, it could be argued that "red" cluster extends farther up along its right edge. The third transformed image does not show this a possible explanation for the improved clustering for this period. The fourth clustering looks a bit different. The walks by the shorter person seem to have been
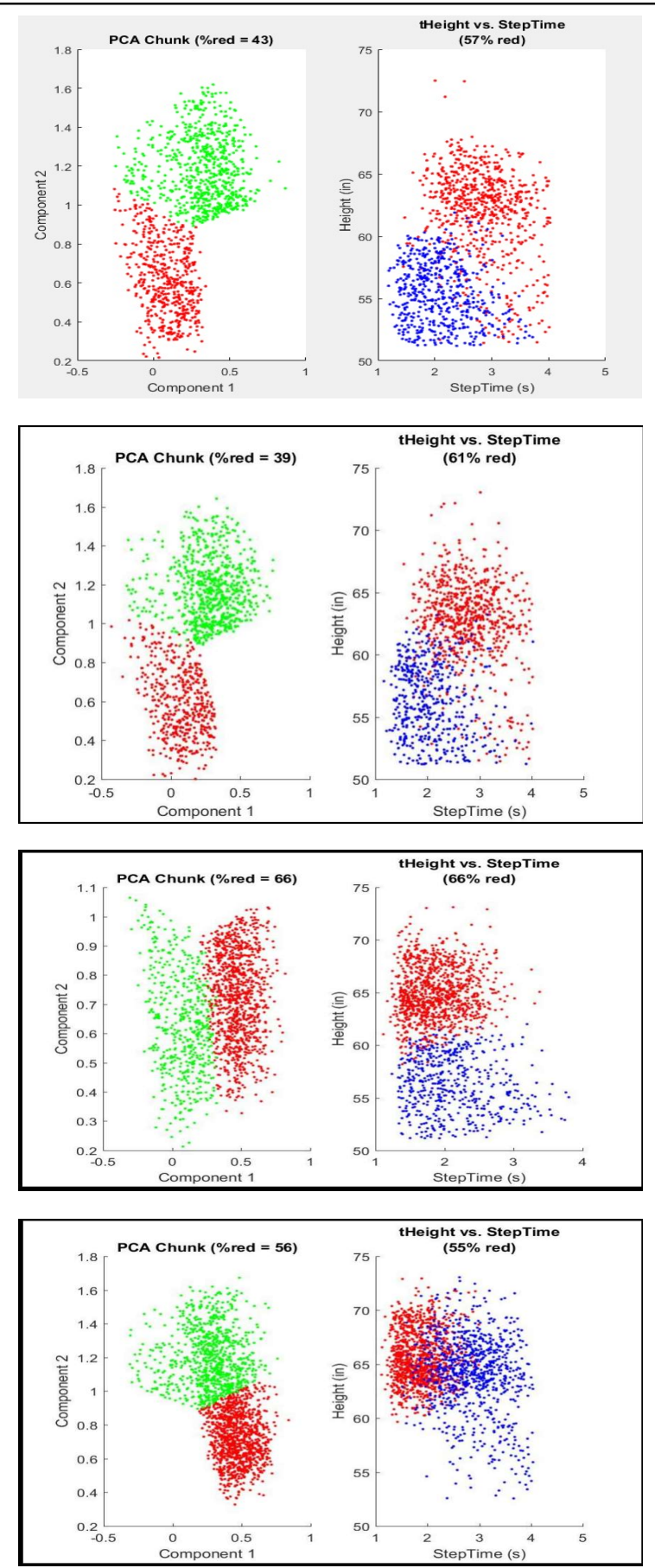

Figure 4-4: Progression of two week chunks clustered using PFCM with PCA transform. The images on the left are PCA transformed dataset with the two most dominant components. The images on the right are the Total Height vs. Step Time metrics. The red clusters are the same resident. 
significantly decreased in number to the point where the algorithm is attempting to split the taller resident's cluster in half. This phenomenon also appeared in the weeks 11 and 12, and returned to "normal" after that. In all four sets of images, the PCA transformed image shows a clean separation between two clusters. The images shown here are representative of all of the residents.

Integrating the clustering approach with the medical history of the residents may provide additional knowledge about the presence or absence of any of the residents and might help improve clustering if this behavior is truly related to one resident being "away from home" during the period. Additionally, the resident's history can also be used to tune the clustering further. For example, if you already know one subject wears a lift which adds asymmetry to their gait, and their partner does not wear a lift, but has a faster walking speed, then you could weight features to emphasize these two metrics in clustering. 


\section{Chapter 5 Predictive Analysis}

\section{Motivation}

As mentioned in chapter 1 , one of the goals of developing centroid based metrics is the ability to reliably monitor subjects, in their homes, and identify changes in their gait that could portend potential falls or other clinical events. This chapter discusses the development of a method of identifying significant changes in a subject's metrics over time which could flag possible falls or other clinical events.

The strategy is to monitor long term trends and identify sudden changes in one or more metrics. In the absence of any significant events, a person's gait should remain steady or slowly decline due to age and/or disease progression. If the metrics are scanned in small chunks, the long-term changes in the person's gait can be eliminated. Any sudden change in a person's gait would generate an outlier or sudden change in the data. The objective, then, is to identify outliers in the monitored metrics and prove the hypothesis that outliers correspond to significant changes in physical conditions and can predict clinical events.

\section{Conditioning of Data}

For this analysis, we will start with data clustered as described in chapter 4. From each metric, a signal showing the rate of change of the minimum detectable change (MDC) was created. Using the MDC effectively normalized the metric as it reflects the degree to which the metric has changed from a baseline. Using the rate of change of the metric served to amplify the changes to the metric which is what is truly sought.

Specifically, the following approach is done for each metric individually. Daily averages are computed and a baseline for the metric is computed over the first thirty days. From the baseline statistics, the Minimum Detectable Change is computed. Starting with day 31, the 
number of MDC's that the current day deviates from the baseline is computed for the remainder of the days.

The hypothesis for this study is that one large, rapid changes in the metrics over a short period of time are indicative of a heightened risk of significant clinical events. The average metrics would indicate this condition with longer and steeper slope changes than would by typical with statistical variance. To amplify these longer, larger slope changes, the signal will be transformed by using the magnitude of the change in the metric. The MDC signal is scanned looking for a peak or valley. A peak is defined as:

$M(t-1)<M(t)>M(t+1)$

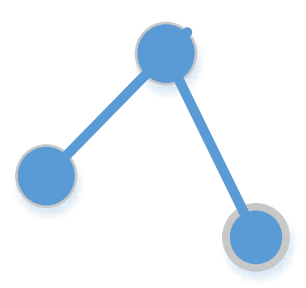

while a valley is defined as

$M(t-1)>M(t)<M(t+1)$

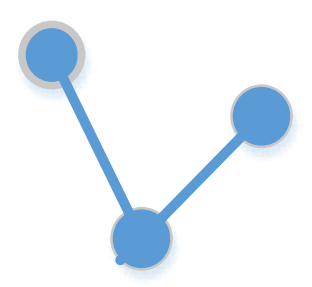

Each point in the original MDC signal is subsequently replaced by the amplitude of the peak-tovalley or valley-to-peak transition that the point is included within. This approach amplifies changes in the metric's signal and factors in the length of time that the slope exists in the signal. This makes it easier to identify outliers as will be described in the next section. 


\section{Outlier Detection using One Class Possibilistic C-Means (OCPCM)}

Outliers were identified using the One Class Possibilistic C-Means algorithm. This algorithm, developed by the Center for Eldercare and Rehabilitation Technology at the Missouri, Columbia and described in Wang [120] uses a modified version of the Possibilistic C-Means (PCM) algorithm. PCM was selected as it is stable with respect to outliers. The algorithm developed here was inspired by Wang's approach and is shown in Algorithm 1. In general, the data is clustered and the possibilities of inclusion in the cluster are generated. The point with the lowest possibility of inclusion is removed and the data is clustered again. This process is then repeated until $50 \%$ of the data points have been removed. After each iteration, the location of the center is logged. Upon completion, the distances between the centers for $\mathrm{n}^{\text {th }}$ and $\mathrm{n}-\mathrm{1}^{\text {th }}$ iteration are computed for all " $n$ ". As each outlier is removed, the cluster center will move very little. As points in the cluster are removed, the center moves more dramatically. The point where the movement of the cluster center becomes more pronounced is the point where members of the cluster are being removed. Looking at the movement of the cluster centers, the average movement of the second half of the removed points is computed. The threshold to separate outliers from points within the cluster was chosen by experimentation to be $75 \%$ of this after movement. This threshold was determined experimentally to strike the best balance between true positive and

1. With full dataset, run OCPCM algorithm to obtain list of possibilities

2. Store location of cluster center

3. Repeat until $50 \%$ of data has been removed.

4a. Remove point with lowest possibility and re-run OCPCM

4b. Store distance center moved due to point deletion

5. Find mean of center movements for second half of removed points

6. Identify the first point that is $>75 \%$ of this value.

7. Label all points with a lower possibility as outliers.

Algorithm 1: Outlier identification using OCPCM. Steps 5-7 reflect an adaptation of Wang's algorithm to this application. 
false positives. All points with an inclusion possibility less than the point at this threshold are classified as outliers.

Outlier detection was also performed on two week chunks of data instead of the entire data set. With some data sets spanning 30 months, it is necessary to break the data into chunks to minimize the effects of long term changes in the subject's physical condition. Also, instead of using the raw metrics, the rate of change of the normalized metric was used. We wanted a more dramatic change to be flagged as an event and using the number of MDC values as the normalization amplifies these changes. This is done by first computing a baseline mean, standard error about the mean, and Minimum Detectable Change (MDC) over the first 30 days. For the remaining points, the number of MDCs that the point deviates from the baseline mean is computed. The daily mean of the slopes of the MDC curve for each walk during the day are computed and used to identify outliers.

This approach was done on a set of features optimized for each subject. The specific algorithm for selecting the feature set is described in the "Metrics Selection" section below.

\section{Scoring Result}

Once outliers have been identified, they were compared to actual clinical data. The

$$
\begin{aligned}
& \text { history of the subject's clinical } \\
& \text { events, limited to falls, emergency } \\
& \text { department visits, and } \\
& \text { hospitalizations, was obtained for } \\
& \text { each resident. The outliers } \\
& \text { identified in the previous section } \\
& \text { were compared to these physical }
\end{aligned}
$$

Table 5-1: Classification criteria for scoring outliers against clinical events.

\begin{tabular}{|l|l|}
\hline Classification & Criteria \\
\hline True Positive & $\begin{array}{l}\text { Clinical event occurs within seven days } \\
\text { after an outlier is identified. }\end{array}$ \\
\hline False Positive & $\begin{array}{l}\text { No clinical event occurs within seven } \\
\text { days after an outlier is found. }\end{array}$ \\
\hline False Negative & $\begin{array}{l}\text { Clinical event occurs with no outlier } \\
\text { within the previous seven days. }\end{array}$ \\
\hline Post Event & $\begin{array}{l}\text { An outlier occurs within seven days } \\
\text { following a clinical event. }\end{array}$ \\
\hline
\end{tabular}


events and scored as shown in Table 5-1. The fourth score category, Post Event, categorizes outliers which follow clinical events. For example, if a resident were to fall and, as a result of the fall, their gait changes due to a sprained ankle, this category would capture that occurrence. Once a clinical event is matched with an outlier, all subsequent outliers that occur within seven days of the clinical event are removed from consideration.

\section{Metrics Selection}

The next task in identifying outliers is to identify the best set of metrics to score each resident. A recurring theme in this research is that each subject has a unique set of metrics and behaviors that are based on their health history, disease progression, typical decline to name a few. It is reasonable to explore the potential differences in results by identifying a unique set of features for each subject. Since a larger feature set is not always the best selection, a set of 16 metrics was chosen initially. These 16 are the best 8 identified earlier when selecting metrics for clustering plus an additional 8 that seemed to produce good iVAT images. Sixteen metrics are used here as the outlier identification is less computationally demanding and for most of the residents, and more than 8 metrics are typically needed for optimum results.

Instead of using a brute force method of checking all 65,535 combinations of metrics for

the best set, the metrics were

eliminated, one by one, using the

method shown in Algorithm 2.

The approach is to start with the

full set of 16 metrics and use the

OCPCM method to identify

outliers. The results are then

scored, and the True Positive and
1. Start with set of Predictive Analysis metrics

2. Run Algorithm 1 on full dataset; compute True Positive score (TP) and False Positive score (FP) Recursive Entry Point (pass data, TP score, and FP score):

3. Repeat for all metrics in dataset.

3a. Remove one metric from dataset.

3b. Run Algorithm 1 and compute TP and FP

4. Select metric set with best TP and FP

5. IF (TP improves) OR (TP stays same, AND FP improves) THEN recursively call passing new dataset and scores. ELSE return passed dataset to caller.

Algorithm 2: Find best set of metrics to identify outliers 
False Positive rates are noted. Next, one metric is removed, outliers are detected, the results scored again, and the metric is replaced. This is repeated for all 16 combinations of 15 metrics and the best score is compared to the starting score. If the percentage of true positives has increased or the percentage of true positives has remained the same and the percentage of false positives has decreased, then the score is considered improved and the process starts again with the best combination of 15 metrics. This process is repeated recursively until the score neither improves nor stays the same. The final set of metrics is used one last time to detect outliers and those outliers are reported.

\section{Results}

\section{Metrics Selection}

A table showing the metrics used for each resident is shown in table 5-2. As expected, each resident had an optimal set of metrics for outlier detection. Average $Z$ entropy is selected for every resident. Left step time, average step time, and average $\mathrm{X}$ entropy are selected in 10

Table 5-2: Chart of the metrics used in the optimal outlier detection. The top row indicates the resident number. $A$ " $X$ " indicates that the metric is included in the resident's optimal set. The numbers in the top row correspond to the resident's apartment numbers with the leading "30" removed. (ie. $04=3004,50=3050$ )

\begin{tabular}{|l|c|c|c|c|c|c|c|c|c|c|c|c|}
\hline Metric & 04 & 12 & 13 & 17 & 37 & 46 & 50 & 52 & 54 & 55 & 57 & Total \\
\hline 10 Foot Walk & $\mathrm{X}$ & $\mathrm{X}$ & & $\mathrm{X}$ & & $\mathrm{X}$ & $\mathrm{X}$ & $\mathrm{X}$ & $\mathrm{X}$ & $\mathrm{X}$ & $\mathrm{X}$ & 9 \\
\hline Average Speed & $\mathrm{X}$ & & & & $\mathrm{X}$ & $\mathrm{X}$ & $\mathrm{X}$ & $\mathrm{X}$ & $\mathrm{X}$ & $\mathrm{X}$ & $\mathrm{X}$ & 8 \\
\hline Stride Time & & $\mathrm{X}$ & & $\mathrm{X}$ & & $\mathrm{X}$ & $\mathrm{X}$ & $\mathrm{X}$ & $\mathrm{X}$ & $\mathrm{X}$ & $\mathrm{X}$ & 8 \\
\hline Left Step Time & $\mathrm{X}$ & & $\mathrm{X}$ & $\mathrm{X}$ & $\mathrm{X}$ & $\mathrm{X}$ & $\mathrm{X}$ & $\mathrm{X}$ & $\mathrm{X}$ & $\mathrm{X}$ & $\mathrm{X}$ & 10 \\
\hline Right Step Time & $\mathrm{X}$ & & $\mathrm{X}$ & $\mathrm{X}$ & $\mathrm{X}$ & $\mathrm{X}$ & & $\mathrm{X}$ & $\mathrm{X}$ & $\mathrm{X}$ & $\mathrm{X}$ & 9 \\
\hline Trunk Sway & & & $\mathrm{X}$ & $\mathrm{X}$ & $\mathrm{X}$ & $\mathrm{X}$ & $\mathrm{X}$ & $\mathrm{X}$ & $\mathrm{X}$ & $\mathrm{X}$ & $\mathrm{X}$ & 9 \\
\hline Average Step Time & & $\mathrm{X}$ & $\mathrm{X}$ & $\mathrm{X}$ & $\mathrm{X}$ & $\mathrm{X}$ & $\mathrm{X}$ & $\mathrm{X}$ & $\mathrm{X}$ & $\mathrm{X}$ & $\mathrm{X}$ & 10 \\
\hline Asymmetry Z & $\mathrm{X}$ & $\mathrm{X}$ & & $\mathrm{X}$ & & $\mathrm{X}$ & $\mathrm{X}$ & $\mathrm{X}$ & $\mathrm{X}$ & $\mathrm{X}$ & $\mathrm{X}$ & 9 \\
\hline Peak to Peak X & & & $\mathrm{X}$ & $\mathrm{X}$ & & $\mathrm{X}$ & $\mathrm{X}$ & $\mathrm{X}$ & $\mathrm{X}$ & $\mathrm{X}$ & $\mathrm{X}$ & 8 \\
\hline Peak to Peak Z & & & & $\mathrm{X}$ & $\mathrm{X}$ & $\mathrm{X}$ & $\mathrm{X}$ & $\mathrm{X}$ & $\mathrm{X}$ & $\mathrm{X}$ & $\mathrm{X}$ & 8 \\
\hline Step Ratio & & $\mathrm{X}$ & & $\mathrm{X}$ & & $\mathrm{X}$ & $\mathrm{X}$ & $\mathrm{X}$ & $\mathrm{X}$ & $\mathrm{X}$ & $\mathrm{X}$ & 8 \\
\hline Average Entropy X & & $\mathrm{X}$ & $\mathrm{X}$ & $\mathrm{X}$ & $\mathrm{X}$ & $\mathrm{X}$ & $\mathrm{X}$ & $\mathrm{X}$ & $\mathrm{X}$ & $\mathrm{X}$ & $\mathrm{X}$ & 10 \\
\hline Average Entropy Y & & $\mathrm{X}$ & $\mathrm{X}$ & $\mathrm{X}$ & $\mathrm{X}$ & $\mathrm{X}$ & $\mathrm{X}$ & $\mathrm{X}$ & $\mathrm{X}$ & $\mathrm{X}$ & & 9 \\
\hline Average Entropy Z & $\mathrm{X}$ & $\mathrm{X}$ & $\mathrm{X}$ & $\mathrm{X}$ & $\mathrm{X}$ & $\mathrm{X}$ & $\mathrm{X}$ & $\mathrm{X}$ & $\mathrm{X}$ & $\mathrm{X}$ & $\mathrm{X}$ & 11 \\
\hline Average Entropy XY & $\mathrm{X}$ & $\mathrm{X}$ & & & & $\mathrm{X}$ & $\mathrm{X}$ & $\mathrm{X}$ & $\mathrm{X}$ & $\mathrm{X}$ & $\mathrm{X}$ & 8 \\
\hline Step Length & & $\mathrm{X}$ & & $\mathrm{X}$ & & $\mathrm{X}$ & $\mathrm{X}$ & $\mathrm{X}$ & $\mathrm{X}$ & $\mathrm{X}$ & $\mathrm{X}$ & 8 \\
\hline
\end{tabular}


(91\%) of the residents.

Step time and entropy

show up as the most

commonly selected

metrics suggesting they,

either individually or in

tandem, are most

sensitive to changes or

most likely to change

preceding a clinical event.

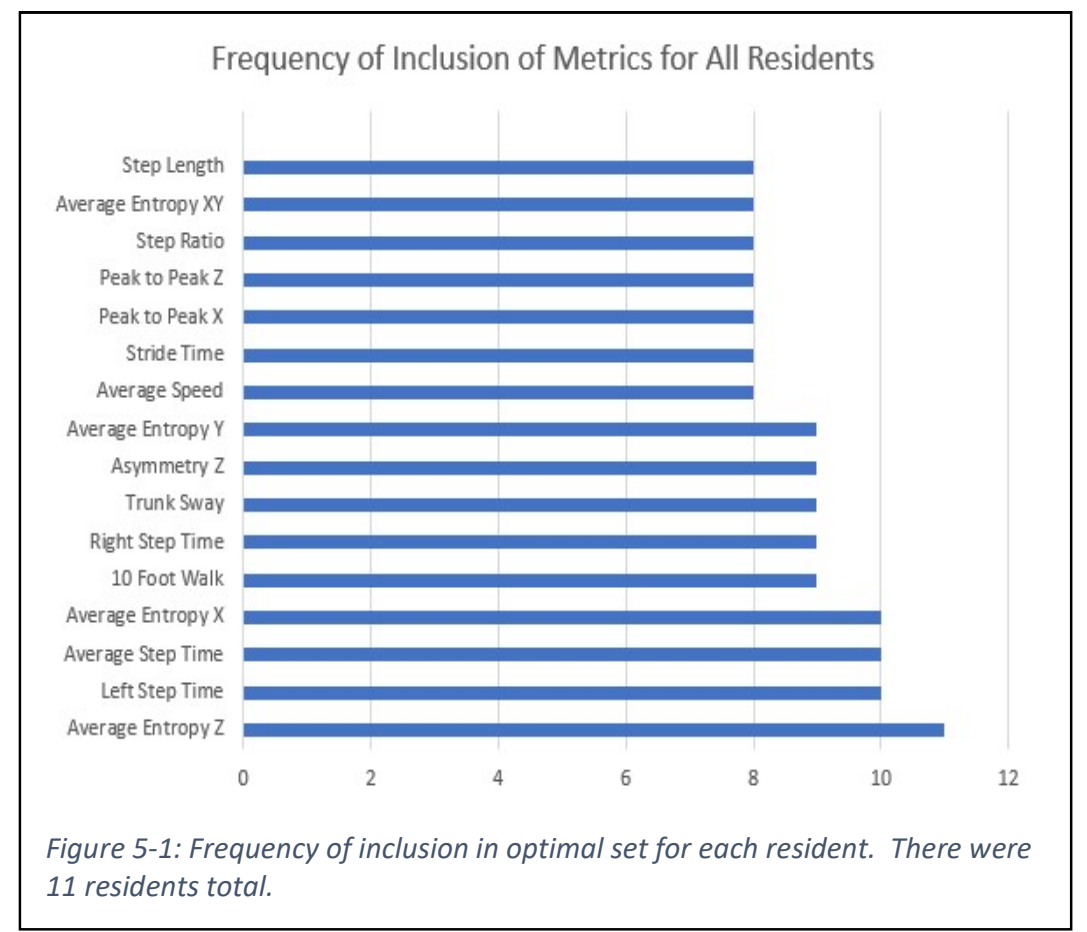

For example, resident

\#3004, who only needed 7 metrics for optimal predictive analysis, used two entropy measurements, two speed metrics, and two step time measurements along with asymmetry. This is also shown graphically in Figure 5-1. 


\section{Outlier Detection and Scoring}

Outlier detection for eleven residents was done and the results are shown in Table 5-3.

Residents 3046, 3052, 3054, and 3055 do not have any clinical events on record and, subsequently do not have any scores for false positive and change. Resident \#3037 has the best score of $80 \%$ true positive with $7 \%$ false positive. $75 \%$ of the clinical events resulted in a significant change to the metrics. Resident \#3012 ended up with a score of $18 \%$. This resident fell several times per

Table 5-3: Scores for eleven residents. M/F shows resident's gender. \#Metrics indicates the number of metrics used for this resident. The "Correct" column shows the percentage of correctly identified points (TruePositive + True Negative). "Post" shows the percentage of clinical events which are followed by a significant change in metrics within seven days.

\begin{tabular}{c|c|ccccc} 
& $\mathrm{M} / \mathrm{F}$ & \#Metrics & TruePos & FalsePos & Correct & Post \\
\hline 3004 & $\mathrm{M}$ & 7 & $60 \%$ & $16 \%$ & $83 \%$ & $80 \%$ \\
3012 & $\mathrm{~F}$ & 10 & $18 \%$ & $11 \%$ & $80 \%$ & $28 \%$ \\
3013 & $\mathrm{~F}$ & 8 & $25 \%$ & $6 \%$ & $93 \%$ & $75 \%$ \\
3017 & $\mathrm{~F}$ & 14 & $33 \%$ & $25 \%$ & $75 \%$ & $83 \%$ \\
3037 & $\mathrm{~F}$ & 9 & $80 \%$ & $7 \%$ & $93 \%$ & $60 \%$ \\
3046 & $\mathrm{~F}$ & 16 & $\mathrm{~N} / \mathrm{A}$ & $6 \%$ & $94 \%$ & $\mathrm{~N} / \mathrm{A}$ \\
3050 & $\mathrm{~F}$ & 15 & $50 \%$ & $22 \%$ & $78 \%$ & $50 \%$ \\
3052 & $\mathrm{~F}$ & 16 & $\mathrm{~N} / \mathrm{A}$ & $7 \%$ & $93 \%$ & $\mathrm{~N} / \mathrm{A}$ \\
3054 & $\mathrm{M}$ & 16 & $\mathrm{~N} / \mathrm{A}$ & $7 \%$ & $93 \%$ & $\mathrm{~N} / \mathrm{A}$ \\
3055 & $\mathrm{~F}$ & 16 & $\mathrm{~N} / \mathrm{A}$ & $8 \%$ & $92 \%$ & $\mathrm{~N} / \mathrm{A}$ \\
3057 & $\mathrm{M}$ & 15 & $26 \%$ & $17 \%$ & $81 \%$ & $\mathrm{~N} / \mathrm{A}$
\end{tabular}

week and, often, several times per day, for much of the study, each fall a clinical event. Falls this common suggest that falling is "normal" and a significant change in metrics before, or after, each fall is not expected. For the remainder of this section, resident \#3012 will be excluded. Of the remaining scores, two others have a true positive rate greater than or equal to $50 \%$ and 3 have a true positive rate $<50 \%$. More than half of the residents have a false positive score $<10 \%$. Of the residents with clinical events, all but one had a significant outlier following a clinical event. 
Table 5-4: Scores for eleven residents using a fixed set of 16 metrics. M/F shows resident's gender. \#Metrics indicates the number of metrics used for this resident. The "Correct" column shows the percentage of correctly identified points (TruePositive + True Negative). "Post" shows the percentage of clinical events which are followed by a significant change in metrics within seven days.

\begin{tabular}{c|c|ccccc} 
& $\mathrm{M} / \mathrm{F}$ & \#Metrics & TruePos & FalsePos & Correct & Post \\
\hline 3004 & $\mathrm{M}$ & 16 & $60 \%$ & $32 \%$ & $68 \%$ & $40 \%$ \\
3012 & $\mathrm{~F}$ & 16 & $13 \%$ & $10 \%$ & $78 \%$ & $46 \%$ \\
3013 & $\mathrm{~F}$ & 16 & $25 \%$ & $7 \%$ & $92 \%$ & $75 \%$ \\
3017 & $\mathrm{~F}$ & 16 & $17 \%$ & $21 \%$ & $78 \%$ & $83 \%$ \\
3037 & $\mathrm{~F}$ & 16 & $80 \%$ & $40 \%$ & $60 \%$ & $20 \%$ \\
3046 & $\mathrm{~F}$ & 16 & $\mathrm{~N} / \mathrm{A}$ & $13 \%$ & $84 \%$ & $\mathrm{~N} / \mathrm{A}$ \\
3050 & $\mathrm{~F}$ & 16 & $50 \%$ & $23 \%$ & $77 \%$ & $50 \%$ \\
3052 & $\mathrm{~F}$ & 16 & $\mathrm{~N} / \mathrm{A}$ & $7 \%$ & $93 \%$ & $\mathrm{~N} / \mathrm{A}$ \\
3054 & $\mathrm{M}$ & 16 & $\mathrm{~N} / \mathrm{A}$ & $7 \%$ & $93 \%$ & $\mathrm{~N} / \mathrm{A}$ \\
3055 & $\mathrm{~F}$ & 16 & $\mathrm{~N} / \mathrm{A}$ & $8 \%$ & $92 \%$ & $\mathrm{~N} / \mathrm{A}$ \\
3057 & $\mathrm{M}$ & 16 & $11 \%$ & $10 \%$ & $87 \%$ & $95 \%$
\end{tabular}

Table 5-4 shows the same data for the case where all 16 metrics are used, and no optimal search is done. As can be seen from the results, there is some improvement, when a personalized set of metrics is used. This is also shown graphically in Figure 5-2. For each resident, the left two bars show the True Positive rate (higher is better) while the second pair of bars show false positive rates (lower is better). For most residents, there is modest improvement in True Positive rates with a personalized set of metrics while there is a more substantial improvement in the False Positive rate with the specialized set of metrics.

The results show that it is possible to predict clinical events by detecting outliers in the metrics. While the results are not as good as

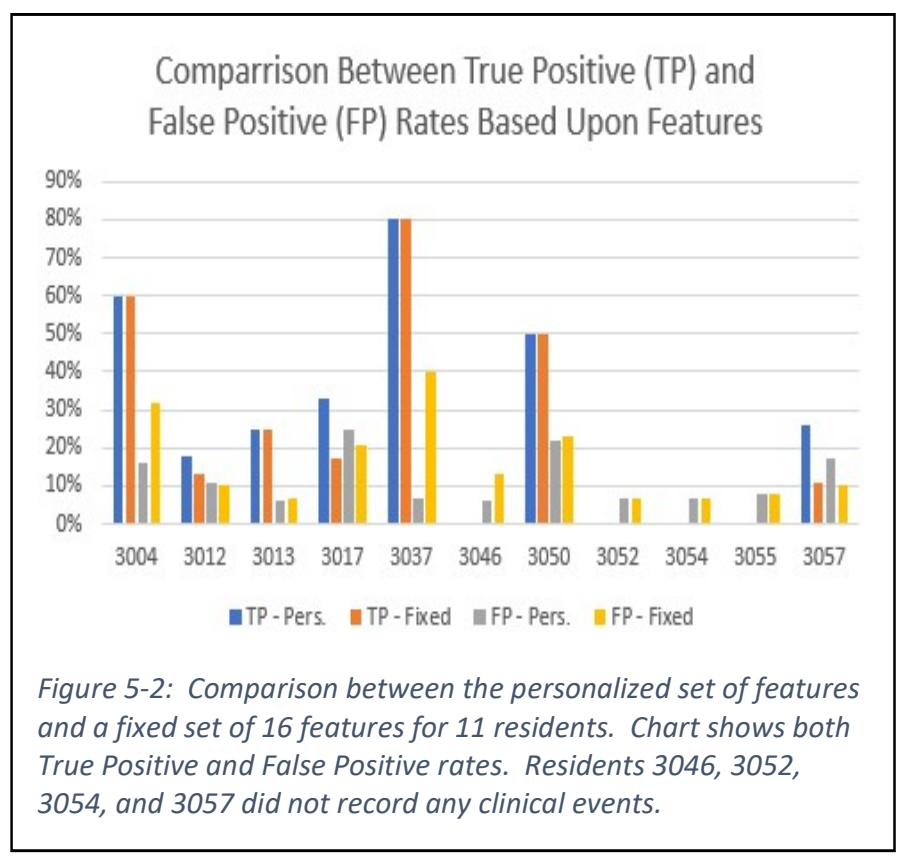
hoped, there are several explanations. The first is the method of separating outliers from 
members of the cluster. In this study, a simple threshold is used. Moving the threshold up (90100\%) will significantly increase the number of false positives and true positives. An area of improvement would be to search for an ideal threshold which maximizes the true positive rate and minimizes the false positive rate for each resident. Doing this may result in better thresholds and better scores. While the actual clinical events are, obviously, in the future, it would be possible to factor in the resident's prior history and use that to improve event detection. In this same vein, utilizing additional data which may have been captured for the resident, alternative measurement modalities, clinical assessments, or other types of monitoring.

A second source of error in this approach is the quality of the clustering. A walk by a visitor which is miss-classified as one done by the resident would have a high probability of being caught here as an outlier and contribute to the false positive rate. This might be improved by looking at each outlier and determining if it is "a little bit out" in which case it might be the resident, or "significantly out" in which case it might be a visitor. Of course, this approach could just as easily call a significant change in the resident's gait as "significantly out" and miss-classify it as belonging to a visitor.

A third cause, and the one most difficult to control, is the capturing a true record of clinical events. In this study, a clinical event is an event recorded in the resident's medical record at the facility. If the resident had a fall which was not severe, or a stumble that did not result in a fall, even though these may have followed outliers in metrics, they would be recorded as false positives. One possible solution to this issue would be to integrate this analysis with automated fall detection[62],[121]. If any of these methods could feed in their detected falls, particularly if they were to identify stumbles, then the scoring could be improved. A second, and more obvious, solution is better recording of falls to capture all falls and stumbles. Lastly, improving the classification of clinical events would improve results. For example, gait related hospitalizations 
are expected to be preceded by outliers while non-gait related hospitalizations should have no relationship to outliers. 


\section{Chapter 6 Trends Analysis}

\section{Motivation}

In addition to identifying potential clinically significant events, the changes to the metrics due to clinically significant events as well as longer term therapies was investigated. The analysis in this case was much more straightforward as a direct comparison was made between each metric both before and after the event to determine if a statistically significant change in the metric was seen.

\section{Methodology}

Non-Vest Related

For this study, the metrics were handled individually. The mean and MDC for the 14 days prior to each event was computed. The mean for the 14 days following the event was also calculated. If the post event mean differed from the pre-event mean by more than $1 \mathrm{MDC}$, then this is considered a significant change.

During this study, it was found that at times, the mean of the metric did not significantly change but the distribution did. MDC can detect some level of change in the variance, but it is possible for the distribution to change significantly without seeing a change greater than $1 \mathrm{MDC}$ in the mean. To detect this scenario, the Kolmogorov-Smirnov (KS) statistical test was used. This test tests for the null hypothesis that both data sets are drawn from the same distribution. If the null hypothesis is rejected with a $95 \%$ confidence, then this is also considered a significant change in the metric.

\section{Vest Related}

A similar test was also done for those subjects who participated in the strategically weighted vest study. In this case, the metrics over the 30 day period after the study was completed (the last time the subject wore the vest) was compared to the 30 day period prior to 
the start of the study (the first time the subject wore the vest). As in the previous non-vest study, both MDC and KS tests were used to judge significance of changes.

\section{Results}

Non-Vest Related

The results from the study with the non-vest residents is shown in table 6-1. Resident \#3012 was excluded, as mentioned earlier, as the frequency of falls through most of the study period was closer to normal behavior than extraordinary and, subsequently, changes in metrics prior to, or subsequent to a fall were not expected. By far, the most often changed metric is stride time changing with $91 \%$ of all clinical events. Five of the next six metrics are all temporal metrics with the Aggregate 8 metric (which has a majority of temporal metrics) as the only non-strictly temporal metric.

While each event was associated with different sets of changed metrics, $100 \%$ of the events were associated with a change in at least one metric. This shows that even with the personalized set of metrics for each resident, stride time does show changes coincident with more than $90 \%$ of events.

\section{Resident \#3012}

During roughly the first $1 / 3$ of the study, resident $\# 3012$ rarely fell. During the rest of the study, this resident fell at least once per week and usually one or more times per day. Using the same algorithm, the one week, three week, and five week periods before and after the first fall were examined. The results are shown in table 6-2. 


\begin{tabular}{|c|c|c|c|c|c|c|c|c|c|}
\hline $\begin{array}{l}\text { Resident \# } \\
\text { (No. Events) }\end{array}$ & $\begin{array}{c}3004 \\
(5)\end{array}$ & $\begin{array}{c}3013 \\
(3)\end{array}$ & $\begin{array}{c}3017 \\
(6)\end{array}$ & $\begin{array}{c}3037 \\
(7)\end{array}$ & $\begin{array}{c}3046 \\
(7)\end{array}$ & $\begin{array}{l}3050 \\
(3)\end{array}$ & $\begin{array}{c}3052 \\
(3)\end{array}$ & $\begin{array}{c}3057 \\
(21)\end{array}$ & $\begin{array}{c}\% \\
\text { Detected }\end{array}$ \\
\hline Stride Time & 100 & 100 & 83 & 86 & 71 & 100 & 67 & 100 & 91 \\
\hline Left Step Time & 20 & & 17 & 14 & 71 & 100 & 67 & 86 & 56 \\
\hline Step Time & 20 & & 17 & 14 & 71 & 100 & 67 & 76 & 53 \\
\hline Rt. Step Time & & 33 & 17 & 14 & 71 & 100 & 67 & 71 & 51 \\
\hline Speed & 20 & & 33 & 43 & 57 & 100 & 67 & 62 & 51 \\
\hline Aggregate 8 & 40 & & 17 & 42 & 57 & 67 & 33 & 61 & 47 \\
\hline $10 \mathrm{ft}$. Walk & 20 & & 33 & 14 & 57 & 100 & 67 & 57 & 46 \\
\hline Entropy XY & & & 17 & 42 & 29 & 100 & 33 & 52 & 39 \\
\hline Aggregate 16 & & & 17 & 42 & 29 & 100 & 33 & 52 & 39 \\
\hline Entropy Y & & 67 & 33 & 14 & 43 & 100 & 33 & 43 & 39 \\
\hline Peak to Peak Y & & & 33 & 14 & 42 & 100 & 33 & 52 & 39 \\
\hline Bounce & & 33 & 17 & 14 & 29 & 67 & 33 & 57 & 37 \\
\hline Peak to Peak X & 80 & 33 & & & 42 & 33 & & 48 & 35 \\
\hline Trunk Sway & & & 17 & 14 & 29 & 100 & & 52 & 33 \\
\hline Asym X & 40 & & & 29 & 29 & 100 & 33 & 33 & 31 \\
\hline Asym Y & 40 & & 17 & & 57 & 100 & 33 & 29 & 31 \\
\hline Peak to Peak Z & & & & 14 & 29 & 67 & 33 & 52 & 31 \\
\hline Avg. Entropy Z & & 33 & & & 43 & & & 52 & 27 \\
\hline Avg. Entropy X & 60 & & & & 29 & & & 43 & 25 \\
\hline Step Length & & & 17 & & 14 & 67 & & 29 & 18 \\
\hline Efficiency & & & 17 & & 29 & 100 & 67 & 10 & 18 \\
\hline Asymmetry Z & 20 & 33 & & 14 & & & 33 & 14 & 13 \\
\hline \% Identified & 100 & 100 & 100 & 100 & 100 & 100 & 100 & 100 & \\
\hline
\end{tabular}

These changes paint a picture of a walk with less oscillation in the $\mathrm{Y}$ direction combined with faster steps and an increase in vertical entropy. An increase in walk efficiency combined with decreases in lateral motion (decreases in Peak to Peak $\mathrm{Y}$, and Entropy $\mathrm{XY}$ ) all suggest a decrease in lateral motion. Combined with an increase in $Z$ entropy this suggests some more randomness (and likely more motion) in the $\mathrm{Z}$ direction. This suggests a more ridged gait with larger vertical and smaller lateral components. Also, there is an initial decrease in stride time and, followed later on, by decreases in average, left, and right step times. With no changes in step length or stride 


length, these suggest an
increase in cadence as
well as in speed.
Looking at the decrease
in ten-foot walk time
supports the general
increase in speed
brought on by the
change in cadence.

Table 6-2: Qualitative changes in 9 metrics over 14, 42, and 70 day periods centered around resident \#3012's first fall.

\begin{tabular}{|l|l|l|l|}
\hline & $\begin{array}{l}+/-7 \text { days } \\
\text { from first } \\
\text { fall }\end{array}$ & $\begin{array}{l}+/-21 \text { days } \\
\text { from first } \\
\text { fall }\end{array}$ & $\begin{array}{l}+/-35 \text { days } \\
\text { from first } \\
\text { fall. }\end{array}$ \\
\hline Walk Efficiency & Increases & No change & Increase. \\
\hline Peak to Peak Y & Decreases & No change & No change. \\
\hline Stride Time & Decreases & Decreases & Decreases \\
\hline Entropy Z & Increases & No change & No change \\
\hline Entropy XY & Decreases & No change & No change \\
\hline 10' Walk Time & No change & No change & Decreases \\
\hline $\begin{array}{l}\text { Step Time } \\
\text { Left Step Time } \\
\text { Right Step Time }\end{array}$ & No Change & No Change & Decreases \\
\hline
\end{tabular}

Lastly, the ten-foot walk speed shows small, but significant changes over this period. A decrease in time implies an increase in speed. While the average speed metric does not show any significant changes, the ten-foot walk time is extended over a longer time period, amplifying any changes to speed.

\section{Vest Related}

Overall effects of the strategically weighted vest therapy were explored next. Using a similar approach as the previous two studies, the means of the metrics for a 30 day period after the therapy concluded were compared to the means for the 30 day period immediately preceding the first time the vest was worn. As in the previous studies, a significant change is defined as a change in mean greater than +/- $1 \mathrm{MDC}$ or a significant difference in the distribution as determined by the KS statistical test.

This was run on the three vest study participants and the results are shown in table 7-3. Roughly 10 months prior to the start of frequent falling, resident 3012 underwent weighted vest therapy. The therapy lasted roughly 2 months, concluding 8 months prior to falling the first time. The therapy reduced walk efficiency by increasing lateral movement ( $\uparrow$ Peak-to-Peak $Y$ and 
个Trunk Sway). Entropy and Peak-to-Peak motion in the $\mathrm{X}$ direction also increased, suggesting more random forward/rearward movement during gait. While the mean stride time did not change, the variance of the stride time measurements did decrease suggesting a more consistent stride time. The resident's walk is described as "stiff and rigid" which is characterized by less flexion in the knee joints during ambulation. Less flexion suggests a higher peak-to-peak motion and entropy in the $Z$ direction and less motion in the lateral direction. The vest therapy appears to have loosened the resident's gait somewhat while reducing the variance in step time.

Resident 3057 showed significant changes in many metrics. A decrease in ten foot walk time and an increase in average speed confirm an increase in speed. A decrease in step times and stride time suggest a faster cadence as well. There was no change in step length implying that the subject is simply walking faster. An increase in all three peak-to-peak measurements along with entropy in the $X, Z$, and $X Y$ directions suggest a walk with more motion in all three directions. This is supported by increases in trunk sway and bounce.

Stride time and all three step times also showed a significant decrease in variance after the vest therapy suggesting the increase in cadence also includes a more regular cadence. Peak to Peak motion in the $X$ and $Y$ directions and entropy in the $X$ direction showed an increase in variance, both suggesting more randomness in these directions. The $Z$ direction, however showed more regularity as the variances for the peak-to-peak and entropy in this direction became more regular.

While an increase in vertical motion is consistent with a tightening of the gait. The increased motion in the $\mathrm{X}$ and $\mathrm{Y}$ directions, however, are more consistent with a looser gait and are more significant than the vertical changes. The resident's average speed has increased due to an increase in their cadence. 
The third participant in the vest study, resident 202, had large increases in all three step time metrics, and a slight decrease in speed. There was also a large increase in step length suggesting longer and slower steps with only a slight change in speed. More motion and randomness in all $\mathrm{X}$ and $\mathrm{Y}$ directions are suggested by increases in peak-to-peak and entropy measurements in these directions. There was no change in either the peak-to-peak Z or bounce metrics, though the variance of these metrics did decrease somewhat.

Table 6-3: Relationship between trends in metrics 30 days prior to, and 30 days following the strategically weighted vest therapy. The MDC columns shows the magnitude of the change in mean expressed in multiples of the MDC value. The KS columns shows the statistical " $p$ " value for the Kolmogorov-Smirnov test. The up or down arrow to the right of the KS value indicates the direction of change in the variance post therapy compared to pretherapy.

\begin{tabular}{|c|c|c|c|c|c|c|c|c|c|}
\hline & \multicolumn{3}{|c|}{ Resident 2} & \multicolumn{3}{|c|}{ Resident 11} & \multicolumn{3}{|c|}{ Resident 12} \\
\hline $10 \mathrm{ft}$. Walk & & & & -1.2 & 0.02 & $\nabla$ & & $<0.001$ & $\nabla$ \\
\hline Avg. Speed & & & & 2.5 & $<0.001$ & $\Delta$ & -1.0 & $<0.001$ & $\boldsymbol{\nabla}$ \\
\hline Efficiency & -1.0 & 0.03 & $\nabla$ & -4.0 & $<0.001$ & $\Delta$ & & 0.007 & $\boldsymbol{\Delta}$ \\
\hline Asymmetry X & -2.0 & $<0.001$ & $\boldsymbol{\Delta}$ & -5.9 & $<0.001$ & $\nabla$ & -3.9 & $<0.001$ & $\boldsymbol{\nabla}$ \\
\hline Asymmetry Y & & & & -4.4 & $<0.001$ & $\nabla$ & & 0.021 & $\boldsymbol{\nabla}$ \\
\hline Asymmetry Z & & & & -1.3 & $<0.001$ & $\nabla$ & & & \\
\hline Step Length & & & & & & & 5.8 & $<0.001$ & $\boldsymbol{\Delta}$ \\
\hline Step Time & & & & -2.5 & $<0.001$ & $\nabla$ & 7.4 & $<0.001$ & $\boldsymbol{\Delta}$ \\
\hline Peak to Peak X & 2.4 & 0.001 & $\boldsymbol{\Delta}$ & 8.0 & $<0.001$ & $\boldsymbol{\Delta}$ & 3.2 & $<0.001$ & $\boldsymbol{\Delta}$ \\
\hline Peak to Peak Y & 1.9 & 0.014 & $\Delta$ & 5.7 & $<0.001$ & $\Delta$ & 2.2 & 0.001 & $\Delta$ \\
\hline Peak to Peak Z & & & & 3.4 & $<0.001$ & $\nabla$ & & 0.003 & $\nabla$ \\
\hline Left Step Time & & & & -2.5 & $<0.001$ & $\boldsymbol{\nabla}$ & 6.6 & $<0.001$ & $\boldsymbol{\Delta}$ \\
\hline Rt. Step Time & & & & -2.5 & $<0.001$ & $\boldsymbol{\nabla}$ & 6.6 & $<0.001$ & $\boldsymbol{\Delta}$ \\
\hline Stride Time & & $<0.001$ & $\boldsymbol{\nabla}$ & -16 & $<0.001$ & $\boldsymbol{\nabla}$ & -2.4 & $<0.001$ & $\boldsymbol{\Delta}$ \\
\hline Bounce & & & & 3.8 & $<0.001$ & $\Delta$ & & 0.01 & $\boldsymbol{\nabla}$ \\
\hline Trunk Sway & 1.4 & 0.003 & $\boldsymbol{\Delta}$ & 5.4 & $<0.001$ & $\Delta$ & 2.4 & $<0.001$ & $\boldsymbol{\Delta}$ \\
\hline Avg. Entropy X & 2.2 & 0.002 & $\boldsymbol{\Delta}$ & 9.8 & $<0.001$ & $\boldsymbol{\Delta}$ & 7.3 & $<0.001$ & $\boldsymbol{\Delta}$ \\
\hline Avg. Entropy Y & & & & & & & & 0.046 & $\boldsymbol{\Delta}$ \\
\hline Avg. Entropy Z & & & & 2.7 & $<0.001$ & $\boldsymbol{\nabla}$ & 1.5 & $<0.001$ & $\boldsymbol{\nabla}$ \\
\hline Avg. Entropy XY & & 0.02 & $\Delta$ & 2.1 & $<0.001$ & $\Delta$ & 3.1 & $<0.001$ & $\boldsymbol{\nabla}$ \\
\hline Aggregate 8 & & & & 6.9 & $<0.001$ & $\boldsymbol{\nabla}$ & & & \\
\hline Aggregate 16 & & & & 3.3 & $<0.001$ & $\boldsymbol{\Delta}$ & & & \\
\hline
\end{tabular}

Overall, increases in peak to peak movement in the $\mathrm{X}$ and $\mathrm{Y}$ directions, trunk sway, and average entropy in the $X$ direction were seen with all three subjects. This raises the possibility that this effect is common with all patients undergoing the strategically weighted vest therapy. 
Peak to peak motion in the $X$ and $Y$ directions, entropy in the $X$ direction, and trunk sway also showed an increase in variance after the vest therapy for all three residents. This behavior could be studied with a larger cohort to determine if this is, in fact, the primary effect the vest has on the patient. 


\section{Chapter 7 Correlation with Fall Risk Assessments}

\section{Motivation}

Part of the standard of care for managing decline in gait and balance is monitoring the subject's gait using one or more fall risk assessments. Several FRA's were described in chapter 2. In this study, the relationship between the developed metrics and the established FRA's is investigated. The FRA's have been shown to be effective in assessing current physical condition as well as identifying changes in a subject's ability to ambulate and possibly risks of falls or other clinical events.

While looking at the result from this study, one must realize that it can be problematic to directly compare FRA's to metrics. Each metric measures only a small component of the subject's gait. For example, an increase in stride time could mean a decrease or increase in speed. If it occurs in isolation, an increase in stride time means a slower cadence with the same step length indicating a decrease in average speed. If the increase in stride time occurs with a proportional increase in stride length, then average speed may increase OR decrease depending upon the degree of change for both metrics. Each metric measures only a single aspect of the subject's gait while the FRA measures the interaction between many different aspects of gait.

\section{Methodology}

A subset of eight subjects had fall risk assessments performed monthly by physical therapists. These fall risk assessments included the instruments described in chapter 2 . The inhome walks are continuously collected during this same time period. As correlation requires similarly sized data sets, metrics were averaged over a three day period centered on the date the fall risk assessments were done. These two datasets were then compared using Pearson correlation. Results were reported if it was strongly correlated $(\rho \geq 0.50)$ with a " $p$ " value $\leq 0.1$. 


\section{Results}

Results from resident \#3004 are presented in chapter \#3.

Results from resident \#3013 are shown in

table 7-1. In this case, the only correlation seen is against the Berg Balance Scale with Peak to Peak

X, Entropy X, Entropy Z, and the Aggregate 8 metric showing negative correlation. In this case, an increase in peak to peak $X$, and entropy in $X$ and Y directions suggest a loosening of the resident's

Table 7-1: Correlations between metrics and FRA's. Only the combinations with strong correlation (>0.5) are shown. The $\Delta$ column shows the change in the metric over the course of the study.

\begin{tabular}{|l|c|c|}
\hline Resident \#3013 & $\Delta$ & BBS \\
\hline Peak to Peak X & $\uparrow$ & -0.57 \\
\hline Avg. Entropy X & $\uparrow$ & -0.60 \\
\hline Avg. Entropy Z & $\uparrow$ & -0.58 \\
\hline Aggregate 8 & $\uparrow$ & -0.62 \\
\hline
\end{tabular}
gait. The negative correlation indicates that the BBS score actually improves over the course of the study. This was verified looking at the actual clinical record for this resident. While there was not a large amount of change in their BBS score, there was a slight trend downward.

For resident \#3053, unlike the previous two residents, there were correlations seen between many metrics and FRA's. The results for this resident are shown in table 7-2. While BBS has shown up in the previous two residents, and SPPB correlation was seen in resident \#3004, neither of these FRA's appear here. In this case, the HGS and TUG fall risk assessments show strong correlation to many metrics. These two FRA's focus more on actual gait and ultimately speed and less on balance. In all cases, for this resident, each of the FRA results show fairly flat trends suggesting minimal changes in these assessments. A strong correlation between these FRA's and metrics also suggest rather flat trends in these metrics. These results may actually indicate that the correlation between the two are not the result of a causal relationship, but merely due to different components of FRA instruments and metrics that do not change. 
Resident \#3050

showed 71 different

pairs showing strong

correlation with 48

(68\%) of the pairs

showing $|\rho|>0.75$.

Even more profoundly

than in the previous

resident, none of the

metrics showed any

significant change in

trends either up or

down over the course of

the study. Perhaps

more so than the

previous resident, this
Table 7-2: : Correlations between metrics and FRA's. Only the combinations with strong correlation (>0.5) are shown. The $\Delta$ column shows the change in the metric over the course of the study.

\begin{tabular}{|l|l|l|l|l|l|}
\hline Resident \#3053 & $\Delta$ & FReach & HGS & TUG & SLS \\
\hline Step Length & $\uparrow$ & & -0.58 & -0.61 & \\
\hline Ten Foot Walk & $\downarrow$ & -0.52 & 0.58 & 0.66 & \\
\hline Average Speed & $\uparrow$ & -0.62 & -0.59 & & \\
\hline Stride Time & $\uparrow$ & & -0.58 & -0.58 & \\
\hline Right Step Time & $\downarrow$ & & -0.51 & -0.52 & \\
\hline Sway & $\downarrow$ & & 0.59 & 0.58 & \\
\hline Step Time & $\downarrow$ & & & & -0.60 \\
\hline Asymmetry Z & $\downarrow$ & 0.51 & -0.53 & -0.62 & \\
\hline Peak to Peak X & $\uparrow$ & & -0.61 & -0.58 & \\
\hline Peak to Peak Z & $\downarrow$ & & & 0.52 & \\
\hline Step Ratio & $\downarrow$ & & 0.59 & 0.63 & \\
\hline Average Entropy X & $\uparrow$ & & -0.61 & -0.58 & \\
\hline Average Entropy Y & $\downarrow$ & & -0.57 & -0.64 & \\
\hline Average Entropy Z & $\uparrow$ & & -0.58 & -0.59 & \\
\hline Average Entropy XY & $\uparrow$ & & -0.58 & -0.58 & \\
\hline
\end{tabular}

resident's results show no significant change in either the FRA or the various metrics over the study period.

Resident \#3052 showed a more modest set of correlations. This is shown in table 7-3. In this case, neither the FReach FRA or the Step Length metric changed significantly over the course of the study. Stride time increased and entropy in the $\mathrm{Y}$ and $\mathrm{Z}$ directions also increased. In this case, the Asymmetry Z decrease coincides with a similar change in BBS. Taken together, these suggest an improvement in this aspect of gait. 
Overall results show

fairly strong correlation

between FRA's and some

metrics for some residents.

A few residents show either

lackluster correlation or the

"flat correlation" seen for
Table 7-3: Correlations between metrics and FRA's. Only the combinations with strong correlation (>0.5) are shown. The $\Delta$ column shows the change in the metric over the course of the study.

\begin{tabular}{|l|c|l|l|l|l|}
\hline Resident \#8 & $\Delta$ & FReach & BBS & TUG & SLS \\
\hline Step Length & - & -0.52 & & & \\
\hline Stride Time & $\uparrow$ & & -0.58 & 0.55 & \\
\hline Asymmetry Z & $\downarrow$ & & 0.60 & -0.69 & \\
\hline Avg. Entropy Y & $\downarrow$ & & & & -0.50 \\
\hline Avg. Entropy Z & $\downarrow$ & & & & 0.56 \\
\hline
\end{tabular}

residents $3050 \& 3053$.

When the data is aggregated, as described in chapter 3, correlation is more apparent. Probably the strongest explanation for this is the scope of each type of measurement. Each FRA is effectively an aggregate of several metrics and measures a wide swath of the subject's condition. For example, the TUG test measures balance (rise from chair, turn around after walking 10 feet, and sitting back down), in addition to gait speed. A TUG score would suffer in the case where the subject has trouble standing and/or sitting but walks easily, as well as when the subject has no trouble standing or sitting but has difficulty walking. The closest FRA to a "pure" metric is the HGS FRA which should correlate well with the Average Speed metric. In this study, however, no significant change is seen in the HGS FRA for any resident. There were changes observed in the Average Speed metric, however. It is possible that the disparity is explained by the measurement accuracy of each data set. For the HGS FRA value, the subject's speed is measured by a physical therapist once during the assessment each month. The Average Speed metric is measured many times per day and averaged over a three day period making the average speed measurement more statistically accurate. Additionally, as mentioned in chapter 1 , the resident's performance could be biased due simply due to their knowledge that their fall risk is being assessed. In the 
case of the metrics, the walks taken in their residence as they perform their daily tasks are measured while there is likely very little thought given to "performing for the camera".

It may be possible to achieve stronger correlation between FRA's and metrics by aggregating metrics to create something which measures something similar to the FRA. For example, if an aggregate that more closely agrees with TUG is wanted, it may be possible to combine weighted contributions of average speed, entropy (to estimate balance), peak to peak movement or trunk sway (to also contribute to balance), or possibly walk efficiency. The other challenge with creating aggregates like these is different subject's physical conditions could result in different combinations of metrics with different weights. This has already been seen several times through this dissertation and would require a large population of monitored residents to achieve. 


\section{Chapter 8 Performance Analysis of Centroid Software}

\section{Motivation}

One motivation for using centroid based metrics instead of measuring gait directly from the movement of the feet is a perceived better result in a cluttered environment, typical of the home environment. This study explores the performance of these metrics compared to an established approach which monitors the movement of the feet. Additionally, as this comparison does not directly explore the impact of occlusions on the effectiveness of these algorithms, a simulated study was done to test the ability of the centroid based algorithms to properly measure walking speed, stride time, and stride length in the presence of these occlusions.

\section{Comparison to Existing Method}

\section{Method}

For a set of five residents, the results of the centroid based metrics were compared against the approach first described in [12] which I will call the "existing method. The existing method will examine each walk and flag walks as invalid if they do not meet certain criteria. These criteria are described in [8] and require the following:

1. The walk must be greater than 1.22 meters in length

2. The walk must be faster than $12.5 \mathrm{~cm} / \mathrm{s}$.

3. The walk must be longer than 1 second in duration.

4. The walk must be "Reasonably straight"

If all four of these conditions are met, the existing method will mark the walk as valid and compute stride time and stride length. Average speed is always computed.

The centroid based metrics were calculated on all walks with a vertical entropy value in the range $[1,10]$. All walks for a particular resident were scanned and average speed, stride length, and stride time were calculated for each walk. Walks not considered valid by the existing method are dropped for the reference algorithm. Walks for which the vertical entropy value is 
out of range are discarded for the centroid based metrics. The final tally of valid walks by each algorithm are compared. Additionally, the distributions of stride time, stride length, and average speed by both algorithms are compared.

\section{Results}

Table 8-1 shows the results of this comparison for six residents. As can be seen, the existing method is only able to calculate metrics for between 11 and $46 \%$ of the walks while the centroid based algorithm can compute between 95 and $98 \%$ of the walks. Calculation of stride length and average speed are fairly close together though stride time has larger discrepancies. While both algorithms validate well against Vicon ${ }^{\circledR}$, there is some disparity between the two during this study. Firstly, the Vicon ${ }^{\circledast}$ data is captured in a controlled laboratory setting. The data used for this study is derived from in home walks captured as described throughout this research. This would likely result in a generally larger error. Secondly, the existing method excludes the first $1 / 2$ second and the last $1 / 2$ second of data from each walk in its calculations while the centroid based metrics do not. This is likely to bias stride length and stride time higher since these walks are less likely to include speeding up and slowing down at the beginning and end of walks. Lastly,

the purposeful walks constraints used by the existing method selects walks that are straighter and more likely to have longer stride times and stride lengths as the subject is walking at "full speed". $\quad$ The centroid
Table 8-1: Results from comparing existing method results to centroid based method. The Total column shows the total number of walks for each resident. The Ref. column shows the percentage of walks for which stride time and stride length are computed with the existing method. The Cent. Column shows the same for centroid based calculations. SL, ST, and AS are the percent difference between the centroid based and existing methods for Stride Length, Stride Time, and Average Speed respectively.

\begin{tabular}{|l|l|l|l|l|l|l|}
\hline Resident & Total & Ref. & Cent. & SL & ST & AS \\
\hline 3004 & 68,073 & $46 \%$ & $98 \%$ & $7 \%$ & $16 \%$ & $8 \%$ \\
\hline 3012 & 49,495 & $11 \%$ & $95 \%$ & $20 \%$ & $20 \%$ & $18 \%$ \\
\hline 3013 & 114,826 & $16 \%$ & $97 \%$ & $10 \%$ & $18 \%$ & $9 \%$ \\
\hline 3017 & 27,488 & $26 \%$ & $97 \%$ & $5 \%$ & $15 \%$ & $12 \%$ \\
\hline 3046 & 14,528 & $25 \%$ & $97 \%$ & $2 \%$ & $10 \%$ & $8 \%$ \\
\hline
\end{tabular}


based metrics also include walks closer to walls and other obstacles where the subject may be accelerating, decelerating, or turning. These would reduce all three metrics as the average speeds and dominant frequencies (used by the stride time calculation algorithm) are lower.

Shown in figures 8-1 through 8-4 are probability density functions of stride length, stride time, and average speed for the existing method (indicated as "GT") and the centroid based method (indicated as "BWF"). From right to left you can see stride length, stride time, and average speed. Comparing the top and bottom figures for each metric, a similar structure can be seen. The existing method tends to have less variance and a generally tighter PDFs than the centroid based algorithm. In light of the sources of discrepancies between the two sets of results, this is not unexpected.

Generally speaking, these results show positive performance when compared against the existing method. The centroid based algorithms, for the subjects tested, will analyze $95 \%$ or more of the walks vs. between 11 and $46 \%$ of the walks for the existing method. While there are some discrepancies between the values of stride length, average speed, and, in particular, stride time, these are likely an artifact of the less restrictive constraints with the centroid based calculations combined with the measurement of full walk. 

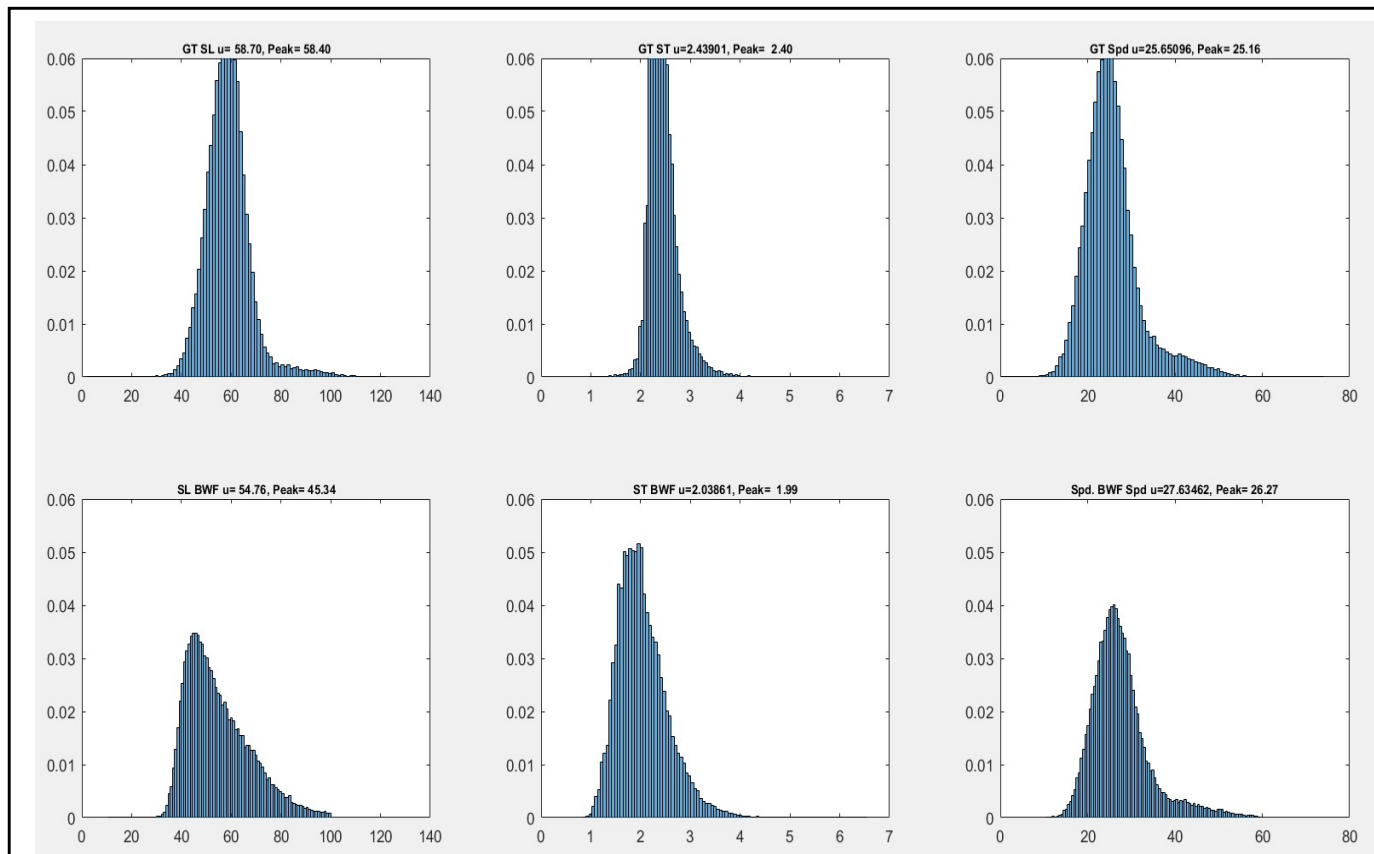

Figure 8-1: PDF's for Stride Length (left), Stride Time (center), and Average Speed (right) for resident 3004. The existing method distribution is on top while the centroid based distributions are on the bottom.
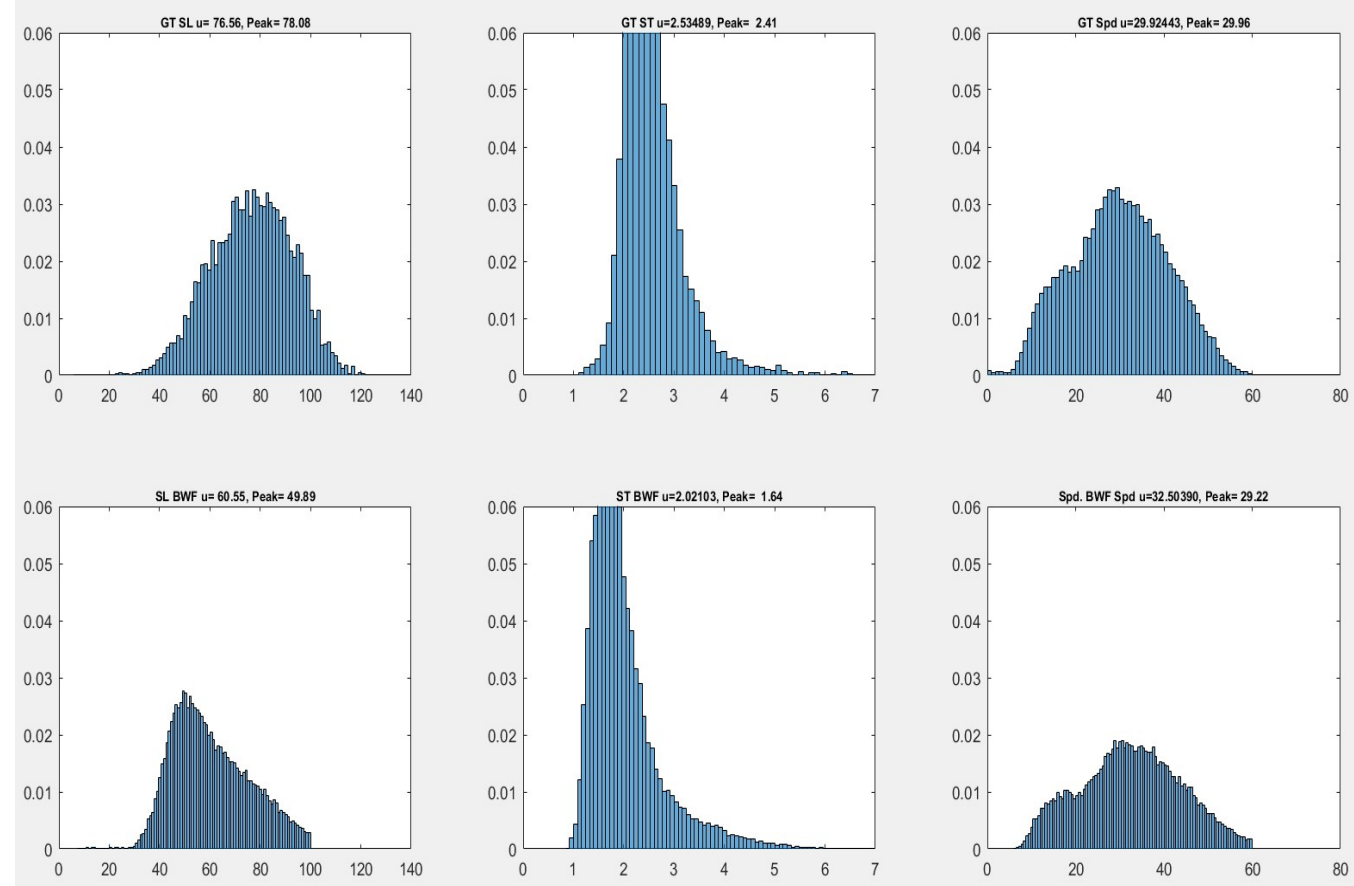

Figure 8-2: : PDF's for Stride Length (left), Stride Time (center), and Average Speed (right) for resident 3012. The existing method distribution is on top while the centroid based distributions are on the bottom 


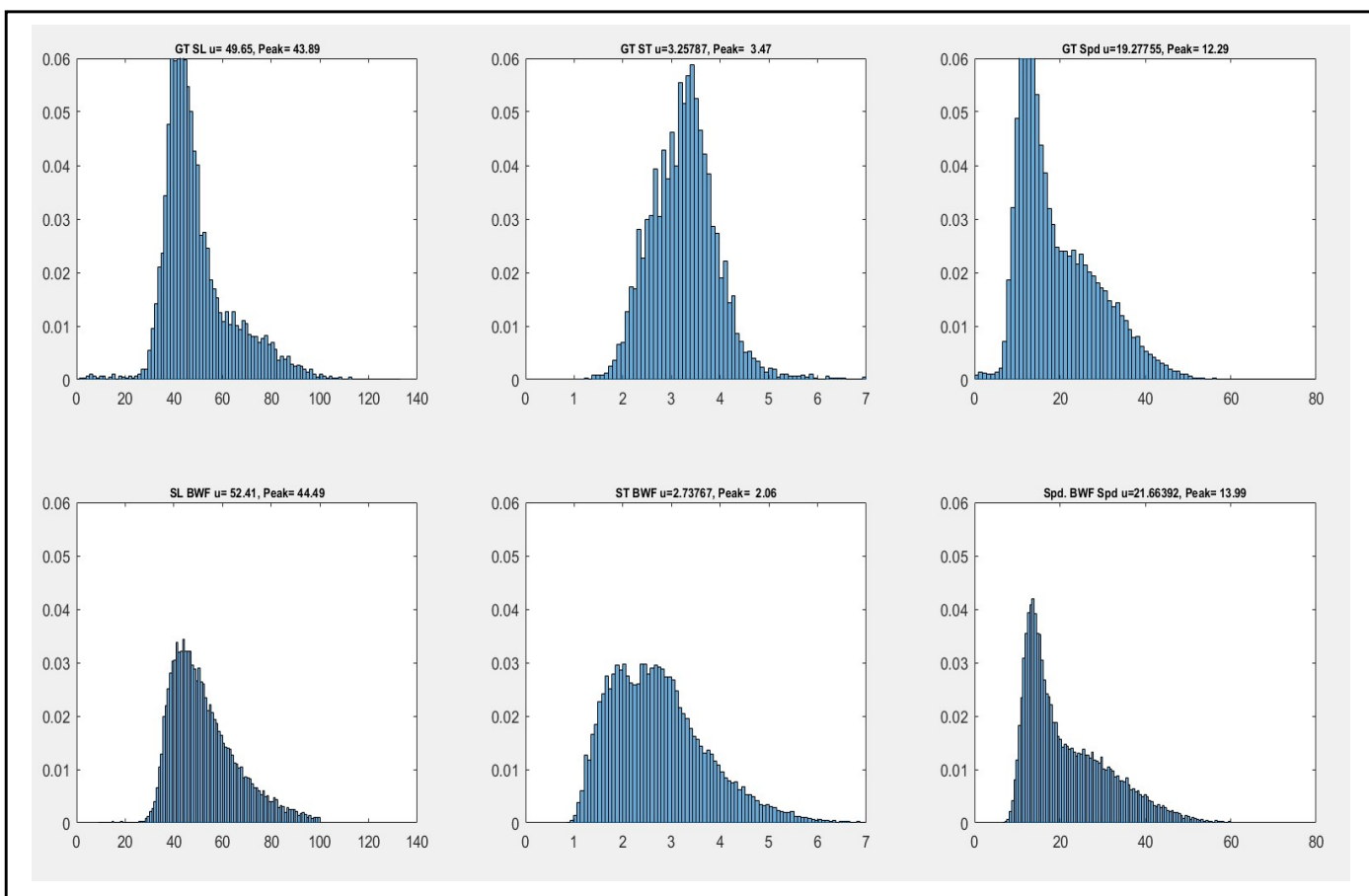

Figure 8-3: PDF's for Stride Length (left), Stride Time (center), and Average Speed (right) for resident 3017. The existing method distribution is on top while the centroid based distributions are on the bottom
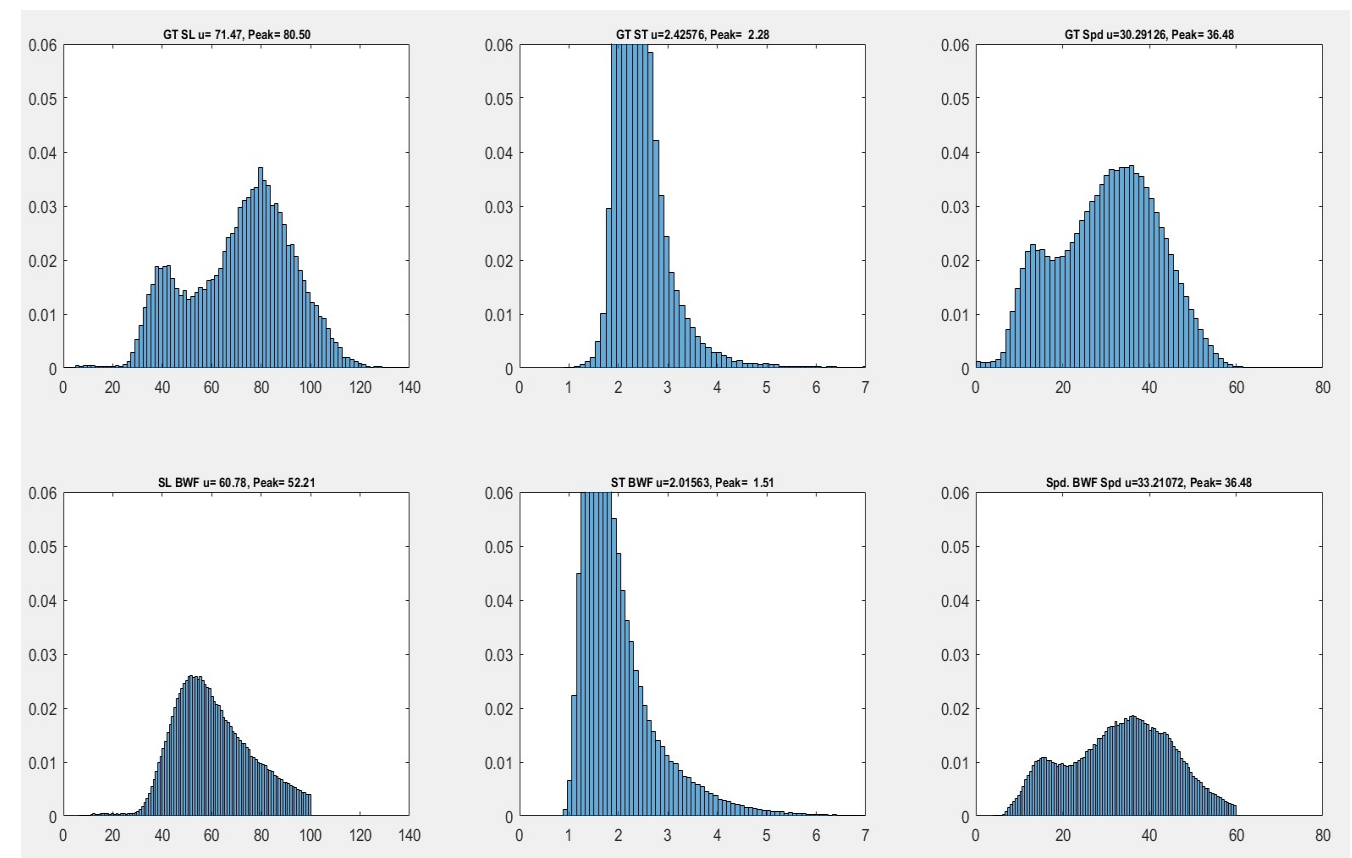

Figure 8-4: PDF's for Stride Length (left), Stride Time (center), and Average Speed (right) for resident 3057A. The existing method distribution is on top while the centroid based distributions are on the bottom 


\section{Simulated Obstruction}

Method

The previous section does not, however, answer the question "How well do the centroid based metrics perform with obstacles in the walking path. The previous study focused on the performance across a wide variety of factors including speed, path, obstructions, and length of time. To answer the specific question of how the centroid based metrics perform around obstructions, a simulated study was conducted as real data did not exist and would be obtrusive to do in a home setting.

A cube measuring $2^{\prime} \times 2^{\prime} \times 2^{\prime}$ was simulated in the middle of the resident's apartment. This was done by defining a $2^{\prime} \times 2^{\prime}$ square on the floor and raising the centroid height by 1 foot whenever the centroid appeared in this square. To illustrate this, let's assume the subject is six foot tall. Their centroid, when not obstructed, will be approximately 3 feet below the top of the point cloud (top of the subject's head). Now, let's insert a cube with 2 foot sides in front of the subject. The lowest 2 feet of the subject's point cloud is now obstructed. When the static components of the image are removed during pre-processing, the resulting point cloud will only be 4 feet tall. This places the centroid two feet below the top of the point cloud, raising the centroid by 1 foot.

The code that generates the metrics was modified such that a 2 foot square area was defined in the middle of the visible area. For every centroid with $X$ and $Y$ coordinates within the square area, one foot in height is added to the $Z$ value. Once this is done, the resulting signals are processed as usual to compute a full set of metrics. The metrics for stride time, stride length, and average speed were looked at and compared to the same sets of unobstructed walks. 


\section{Results}

The results of this study

are shown in table 8-2. In all cases, stride length and average speed showed no impact from the simulated obstruction. Stride Time showed between 0 and $4 \%$ change. This could be due to a slight increase in error when computing the dominant
Table 8-2: Results from comparing existing method results to centroid based method. The Total column shows the total number of walks for each resident. The Ref. column shows the percentage of walks for which stride time and stride length are computed with the existing method. The Cent. Column shows the same for centroid based calculations. SL, ST, and AS are the percent difference between the centroid based and existing methods.

\begin{tabular}{|l|l|l|l|l|l|}
\hline Resident & Total & Ref. & SL & ST & AS \\
\hline 3004 & 76,147 & $45 \%$ & $0 \%$ & $1 \%$ & $0 \%$ \\
\hline 3012 & 60,121 & $49 \%$ & $0 \%$ & $0 \%$ & $0 \%$ \\
\hline 3013 & 114,798 & $38 \%$ & $0 \%$ & $4 \%$ & $0 \%$ \\
\hline 3017 & 31,184 & $25 \%$ & $0 \%$ & $3 \%$ & $0 \%$ \\
\hline 3037 & 81,010 & $18 \%$ & $0 \%$ & $3 \%$ & $0 \%$ \\
\hline
\end{tabular}

frequency in the $Z$ centroid movement. In this simulated environment, walking behind the "obstruction" causes the centroid to move the entire one foot upward within one frame. This results in a step in the $Z$ centroid movement. As with any step function, there is energy at all frequencies which, when the FFT is taken, could result in a higher dominant frequency and lower stride time. This is borne out in the variance of the stride time computation. In the obstructed data, the variance of the stride time increases by as much as $60 \%$. The probability density functions confirm this. While all of the PDF images have the same characteristics both with and without the obstruction, the stride time PDF's do tend to look a bit more ragged. The PDF images are shown in figures 8-5 through 8-8. 

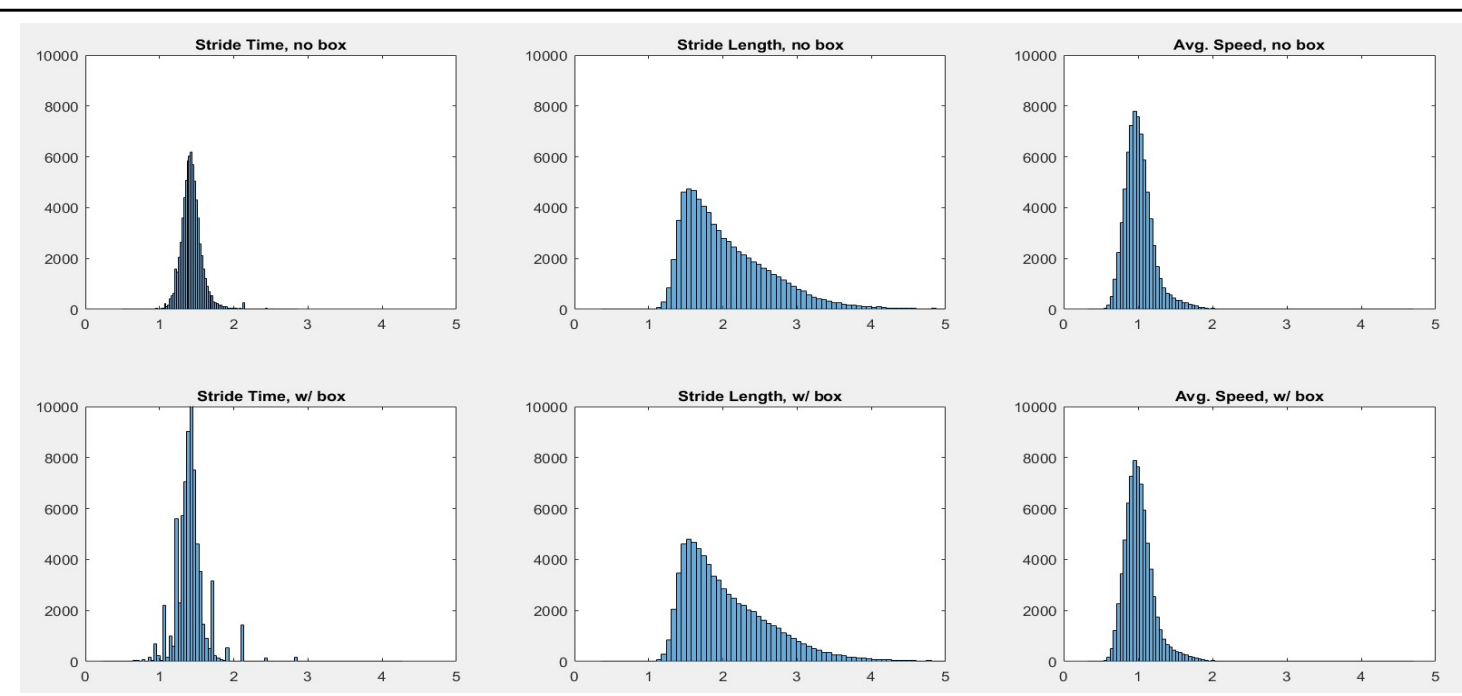

Figure 8-5: PDF's for resident 3004. Probability density functions for the simulated obstruction. The top row shows the PDF's for Stride Time, Stride Length, and Average Speed without the simulated obstruction. The bottom row shows the same images for the dataset with the included simulated obstruction.
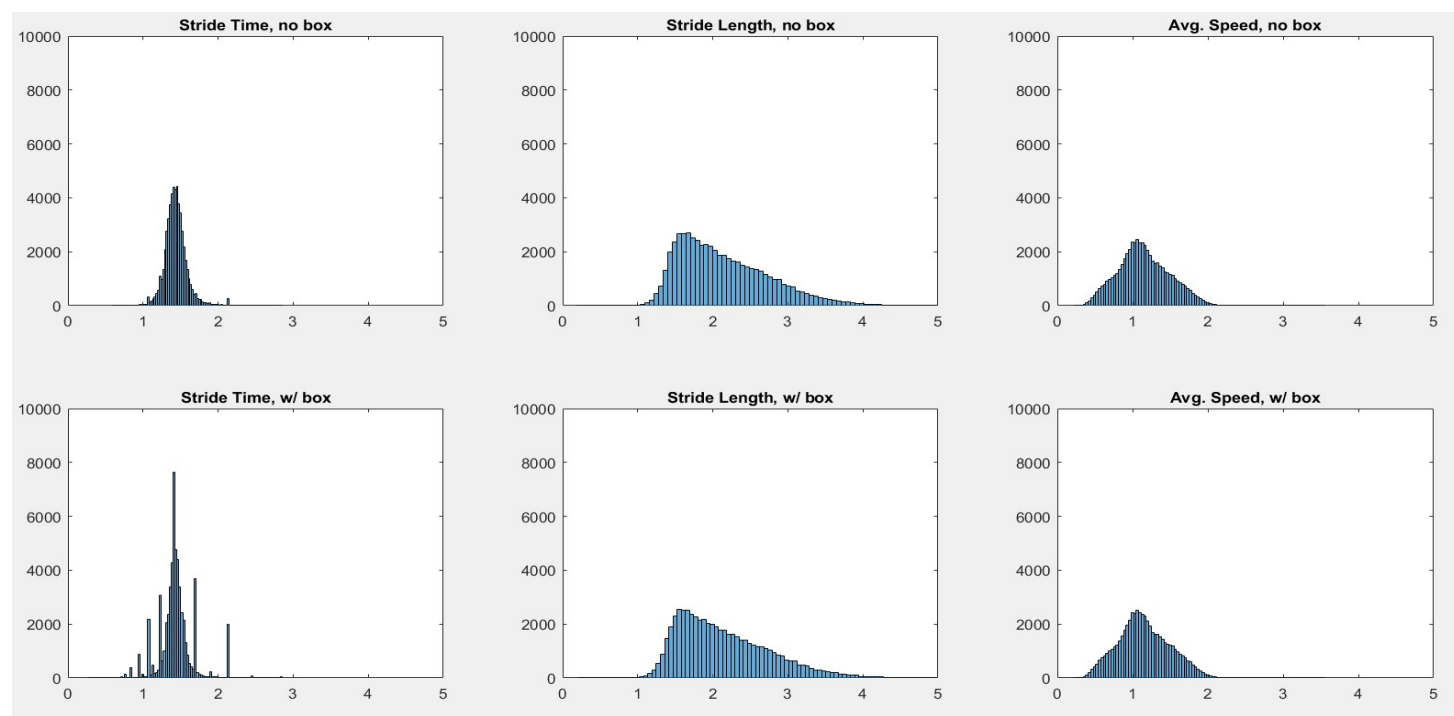

Figure 8-6: PDF's for resident 3012. Probability density functions for the simulated obstruction. The top row shows the PDF's for Stride Time, Stride Length, and Average Speed without the simulated obstruction. The bottom row shows the same images for the dataset with the included simulated obstruction 

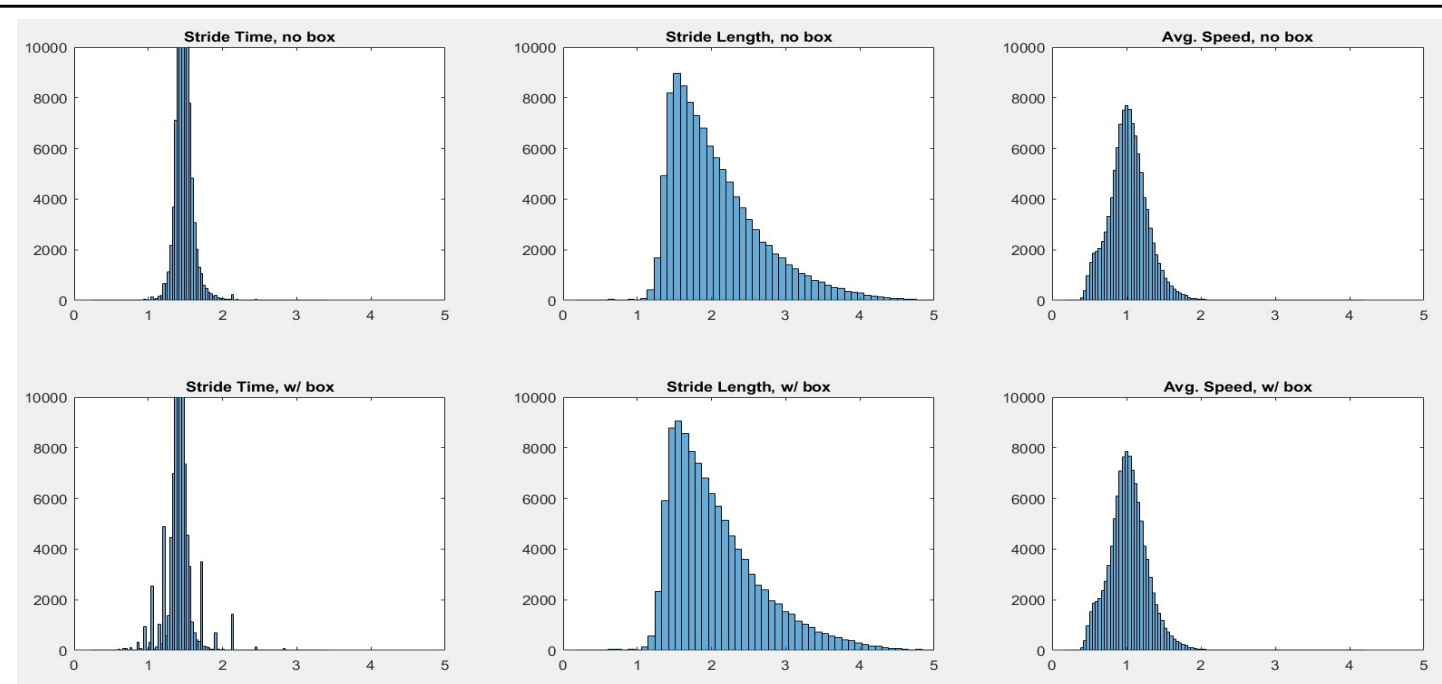

Figure 8-7: PDF's for resident 3013. Probability density functions for the simulated obstruction. The top row shows the PDF's for Stride Time, Stride Length, and Average Speed without the simulated obstruction. The bottom row shows the same images for the dataset with the included simulated obstruction
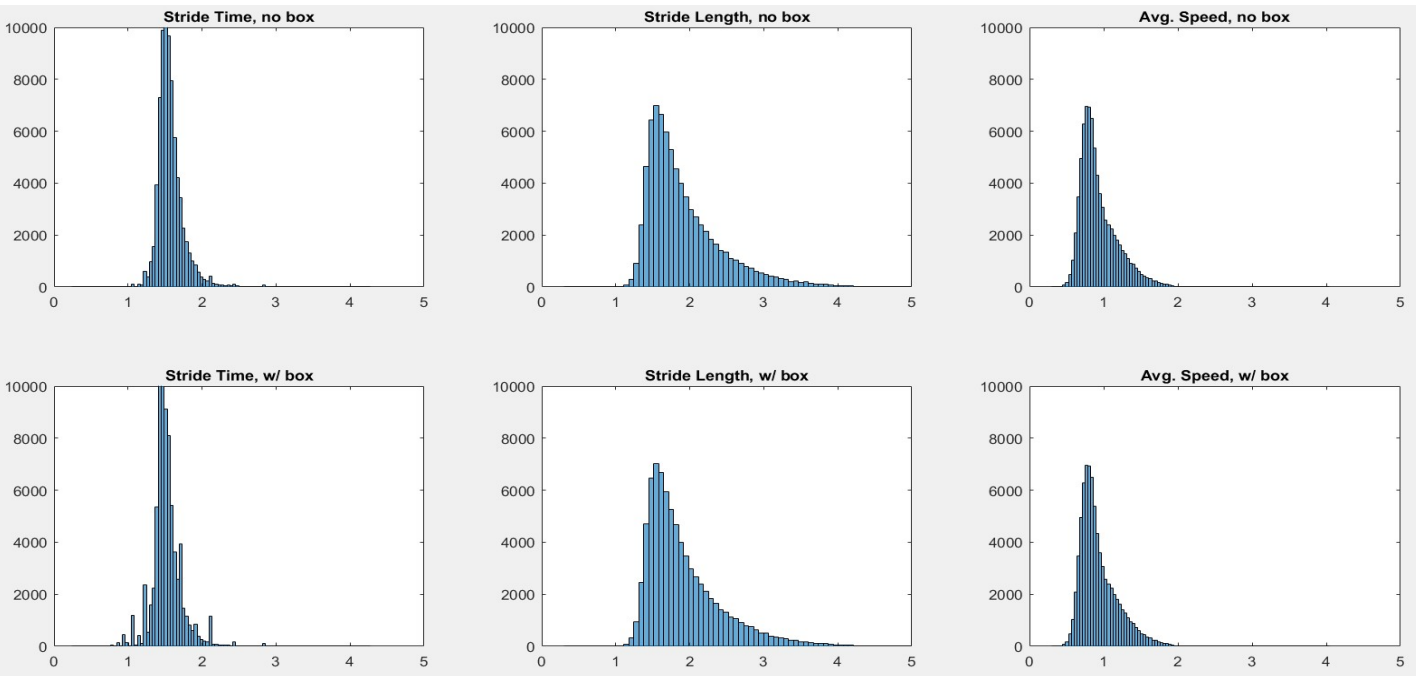

Figure 8-8: PDF's for resident 3037. Probability density functions for the simulated obstruction. The top row shows the PDF's for Stride Time, Stride Length, and Average Speed without the simulated obstruction. The bottom row shows the same images for the dataset with the included simulated obstruction 


\section{Chapter 9 Overall Summary of Results}

While many results and conclusions have been shared in the specific chapters, this section summarizes the most salient conclusions in one place and tie each chapter together to show how these centroid based metrics can be used to characterize gait and detect significant changes either due to clinical events, or that may predict a clinical event.

\section{Verification and Validation}

Chapter 3 discusses the verification of stride time and stride length metrics against an independently developed and validated ground truth algorithm using laboratory generated data with 13 subjects ( 8 walks $\times 2$ cameras each). Based upon the results of the verification obtained, the algorithms can compute, on average, stride time to within $2 \%$ of the values computed by the Vicon ${ }^{\circledR}$ system.

Validating these algorithms against in home collected data showed similarly good results. As also mentioned in Chapter 3, peak error for stride time, left step length, and right step length error versus the Ground Truth Algorithm shows a mean error less than $4 \%$ for 6 of the 7 subjects. The $7^{\text {th }}$ subject has a significantly asymmetric gait due to hip surgery and orthotic lifts on one side. The Probability Density Function for the error shows two peaks in the ground truth algorithm. A primary peak aligned with the peak in the proposed algorithm, and a second peak to the right (longer step time). It is this secondary peak that is skewing the stride time measurements. This illustrates one weakness in the algorithm - if the subject's gait is temporally asymmetric, there is energy in the Fourier Transform at two frequencies which could lead to incorrect calculations of the step time. This could skew the remainder of the temporal metrics as they all depend upon 
step time. A reasonably simple solution to this may be to detect two distinct peaks in the FFT and do a weighted average based upon the area of each peak to compute a better estimate of the dominant frequency.

Another weakness was noted in the previous chapter. One benefit of using centroid based metrics is the ability to compute metrics over a much larger set of walks. Relaxing the constraints imposed by the existing method also adds some additional error. As these centroid based metrics include the first and last parts of the walk, as well as walks where the subject may not be walking in a "reasonably straight" path, there may be more variability as the subject may be accelerating, decelerating, or turning. In the studies shown here, absolute accuracy is not as important as being able to detect relative changes. If absolute accuracy is needed, applying the purposeful walks criteria and clipping the first and last $1 / 2$ second from each walk would remove much of this error.

In spite of the weaknesses, this does outline a set of metrics that can be, and have been, used to monitor a subject's ambulation. These metrics are validated against both the VICON ${ }^{\circledR}$ system as well as a existing method.

\section{Performance}

The most dramatic result from this study is the percentage of walks that can be processed by the centroid based metrics versus the existing method. The existing method was only able to compute $11-46 \%$ of the walks for the set of residents studied. The centroid based metrics could be computed for greater than $95 \%$ of the walks. As mentioned earlier, the centroid based walks showed the same shape in the PDFs, but shifted downward slightly. These are likely to be due to a wider range of walks being included as well as the entire walk being used instead of a shortened walk. This is supported by the tendency for the means to be shifted to a slower speed and shorter 
steps (lower stride time) which is an expected set of changes as a person accelerates or decelerates. This is beneficial as walks captured in the cluttered home environment may be more susceptible to noise, obstructions, other residents or visitors, or a number of other causes for invalid data which must be excluded. By starting with a larger number of walks, removing invalid walks matter less to the overall results.

The simulated obstruction study showed that the metrics are minimally impacted by having a physical obstruction in the camera's field of view. In fact, the Stride Length and Average Speed parameters showed no error for all five of the residents examined. Stride Time did show between 0 and $4 \%$ error with between 18 and $49 \%$ of the walks obstructed. The percent error was not related to the number of obstructed walks as the apartment with the most obstructed walks also had the lowest stride time error (0\%).

These results show, when combined with the results of the metric validation, that it is not only possible to use centroids to estimate stride time, stride length, and average speed, but that performance is as good as the existing method with nearly double the number of walks which can produce results. This can be particularly important if the subject only has a limited number of walks per day due to their physical condition or habits.

\section{Clustering}

Developing an improved method of separating the walks from different residents in the same apartment was necessary in a few apartments. Results show that by utilizing the PCA transformation on the original data, and using dynamic feature selection from a set of high performing features across all residents, good clustering can be obtained. The prior method of using a two or three cluster Gaussian Mixture Model, while somewhat effective on many 
apartments, failed on a few apartments. Clean separation for all apartments was seen with the PCA / PFCM approach.

Using dynamic feature selection increased the effectiveness of the clustering. In many cases, a smaller feature set produced better results than the full set of 8 metrics. This is, likely, due to the similarities between different metrics. Additionally, adding features to a clustering can often make the clustering more complicated as there are more degrees of freedom in the underlying convergence. However, adding additional features can also amplify subtle differences in each individual feature by combining features.

\section{Use With the Weighted Vest}

The early part of this project has shown the basic ability to detect changes in a person's ambulation using centroid based metrics. Results have been presented that show the ability to detect changes in several metrics that are coincident with putting on and taking off the vest. Results also show the ability of the analysis technique and metrics to focus on very short-term effects and longer term effects. For example, by repeating the analysis with several window sizes, it is possible to identify which metrics react the fastest to an event or a therapy, and which ones take longer to react.

This portion of the research has shown that different metrics respond with different latencies following donning and doffing the vest. While this was not done, by plotting the changes in the metrics for different window sizes after the vest is put on or removed, a picture of the relationship between the vest and the several metrics can be observed. The short-term vest study in chapter three did show that, based upon the window size chosen, there is a difference in the behavior of the metrics. 


\section{Predictive Analysis}

I also looked at the ability to detect significant changes in the metrics as harbingers of clinical events. Using the well clustered data, a technique was developed whereby the One Class PCM algorithm was used to identify outliers in the walks. These outliers, it was found, tend to correspond to clinical events. While there was some significant number of false positives as well as, at times, lower true positive scores, these could be due to a non-ideal threshold to identify outliers, errors or missing classifications of clinical events, or small errors in clustering.

In spite of these issues, an ability to predict clinical events was seen using this approach with $60 \%$ to $80 \%$ true positive scores and fairly low false positive scores (typically less than $<15 \%$ with all $<25 \%)$.

Dynamic feature selection also improved the scores. Only four out of 11 residents used all 16 features for outlier detection. The remainder used some subset of the full set. Stride time turned out to be the one feature used by all 11 residents with temporal metrics being used more often than the entropy or basic metrics.

\section{Trend Analysis}

Examining the utility of these metrics for identifying long term changes due to both clinical events and therapy also generated good results. In all of cases, a change in one or more metrics was seen coinciding with a clinical event. Once again, the temporal metrics were the most likely to change along with a clinical event. As mentioned above, change was not limited to changes in the values of the metrics, but also the change in the distribution of the metric. While the mean, for example walking speed, may not change significantly, the subject may still have a more random gait with a higher variance among walking speed values. This was seen in several cases. 
This approach was also used to identify changes due to therapies. While different subjects responded differently to the vest therapy, there were some commonalities. Increases were seen in peak to peak movement in the $\mathrm{X}$ and $\mathrm{Y}$ directions, trunk sway, and average entropy in the $\mathrm{X}$ direction were seen with all three subjects. While it we do not have enough data to draw solid conclusions, this does show that a broader study might show equally promising results and help to explain how the vest therapy actually works.

Lastly, trend analysis was done before and after a major physical change. Resident \#3012, part way through the study, became a frequent faller. The 30 day period before the first fall was looked at and compared to the 30 day period following the first fall. These results show a stiffening of the resident's gait coincident with the first fall.

\section{Relationship to FRA}

Comparing these metrics against existing FRA instruments show correlation between the FRA's and the metrics. Results have shown that while a general relationship does exist between FRAs and the metrics, it is not consistent across different subjects. As each of the FRA instruments measures complex combinations of more basic ambulatory parameters (gait, sway, entropy to name a few), it is possible that a TUG score will increase based upon different mechanisms for different subjects and/or diseases. For example, consider two imaginary subjects. The first subject's walking speed is slowly declining due to age and some arthritis. The second subject, however, can still walk just fine, but has difficulty in the initial rising and final sitting down portion

of the test due to balance issues. While both will experience an increase in their TUG times, they will likely have very different long term changes in their metrics. The first will see a combination of decrease in step length and/or an increase in step time. The second may see an increase in asymmetry, trunk sway or entropy. 


\section{Chapter 10 Status and Future Work}

In this chapter, I'll start by outlining the commitments made at my comprehensive examination and show their current status as well as provide some suggestions as to future work to both develop the metrics as well as improvements to or additional methods of analysis.

\section{\#1 - Develop set of metrics that can be used to track subject ambulation}

This was completed for my comprehensive examination. It has since been published in the Smart Health paper included in chapter 3. One area that may benefit from some additional effort is investigating the existence and usefulness of an aggregate metric. While some investigation of aggregate metrics was done as part of this research, it was not complete nor was it a primary focus. Consequently, only two aggregates were explored - one consisting of 8 most effective metrics with the predictive analysis, and the other using the 16 metrics used for the predictive analysis.

\section{\#2 - Develop analysis techniques to support monitoring short term effects of interventions.}

This was completed for my comprehensive examination. It has since been published in the Smart Health paper included in chapter 3. As mentioned in the paper, one area of further study is the dependency of the short term analysis on the window size. For this study, the window size was chosen to maximize the number of metrics that changed as well as the magnitude of these changes. One short look at a much shorter window for one of the residents showed a different picture as some metrics reacted more quickly after the vest was donned while others took longer to achieve a significant change. By varying window sizes for a series of runs, it may be possible to develop a picture of the temporal behavior of different metrics.

\section{\#3 - Validate metrics against a validated algorithm to verify proper function.}

This was completed for my comprehensive examination. It has since been published in the Smart Health paper included in chapter 3. 
\#4 - Characterize performance of metrics against established FRA's.

This was partially completed for my comprehensive examination. It has since been published in the Smart Health paper included in chapter 3. Additional work is presented in chapter 7.

\#5 - Use metrics and analysis techniques to study short term effects of the strategically weighted vest therapy.

This was completed for my comprehensive examination. It has since been published in the Smart Health paper included in chapter 3. This study would benefit from a broader study involving more people and, perhaps, a longer period of time undergoing the therapy (if appropriate).

\#6 - Validate stride time and step length metrics against the Vicon ${ }^{\circledR}$ system.

This was completed for my comprehensive examination. It has since been published in the Smart Health paper included in chapter 3.

\#7 - Develop a computational methodology to track long term trends in these metrics and detect changes.

This was completed and described in chapters 5 and 6 . A manuscript describing this research is currently being prepared. As described in chapter 5 , this component would benefit from a more intelligent method of establishing the possibility threshold for outliers. Currently there is some variability between the true positive and false positive scores directly due to the threshold. A couple of ideas were mentioned in the chapter including using a dynamic threshold that can be learned from the resident's history. Also, the identification of clinical events can be improved by integrating this module with other modules that perform fall detection.

\#8 - Use metrics and methodologies in \#7 to study long term effects of the strategically weighted vest therapy. 
This was completed and described in chapter 6 . A manuscript describing this research is currently being prepared. As mentioned for \#5, perhaps the biggest benefit to this component would be repeating the study with more residents.

\#9 - Use metrics and methodology in \#7 to study impact of major interventions on two or three subjects. Compare to existing FRA instrument results

This was completed as described in chapters 5 and 6 . A manuscript describing this research is currently being prepared. Some residents had hospitalization events which were investigated as part of chapter 6 . I also looked at the changes in the metrics in resident \#3012 before and after frequent falling started. This could benefit from more frequent FRA assessments (though that may be impractical or an excessive burden to the resident or healthcare staff) to increase the amount of data used in determining correlation. With more data points to consider, existing correlations may be more pronounced and/or additional trends may start to show statistically significant correlation.

\#10 - Use metrics and methodology in \#7 to study long term change in metrics and compare any changes to FRA's.

This was completed as described in chapter 6 (trends analysis) and chapter 7 (fall risk assessments).

\#11 - Determine the fraction of walks that are not included using E. Stone's algorithm due to occlusion which are considered valid with the centroid based metrics. Focus on stride time and stride length.

The fraction of walks that are rejected using E. Stone's algorithm (the "existing method") are compared to the centroid based metrics in the beginning of chapter 8. As the walks that were rejected by the existing method may be rejected for many reasons, including obstruction, a simulated obstruction was added to the data. This is described at the end of chapter 8 . This could be improved by configuring the CERT Motion Capture lab with one or more physical obstructions and re-running the simulated study in chapter 8. 
\#12 - Improve clustering algorithm from currently implemented method (GMM on $n+1$ clusters) to PCM and/or PFCM.

This was completed and described in chapter 4 . In addition to using PFCM, PCA was also used to prepare the data for clustering. 


\section{Appendix I: Summary of Current Status of Proposed Tasks}

NOTE: IEEE = IEEE EMBS [9], SH = Smart Health [10], PH = Pervasive Health [11]

\begin{tabular}{|c|c|c|c|c|}
\hline$\#$ & Description & Status & Chapter & Published \\
\hline 1 & $\begin{array}{l}\text { Develop set of metrics that can be used } \\
\text { to track subject's ambulation. }\end{array}$ & $\begin{array}{l}\text { Completed for comprehensive } \\
\text { exam fall, } 2014\end{array}$ & 3 & IEEE, SH \\
\hline 2 & $\begin{array}{l}\text { Develop analysis techniques to support } \\
\text { monitoring short term effects of } \\
\text { interventions. }\end{array}$ & $\begin{array}{l}\text { Completed for comprehensive } \\
\text { exam fall, } 2014\end{array}$ & 3 & IEEE, SH \\
\hline 3 & $\begin{array}{l}\text { Validate metrics against a validated } \\
\text { algorithm to verify proper function. }\end{array}$ & $\begin{array}{l}\text { Completed for comprehensive } \\
\text { exam fall, } 2014 .\end{array}$ & 3 & IEEE, SH \\
\hline 4 & $\begin{array}{l}\text { Characterize performance of metrics } \\
\text { against established FRA's. }\end{array}$ & $\begin{array}{l}\text { Completed for comprehensive } \\
\text { exam fall, } 2014 .\end{array}$ & 3 & $\begin{array}{l}\text { IEEE, SH, } \\
\mathrm{PH}\end{array}$ \\
\hline 5 & $\begin{array}{l}\text { Use metrics and analysis techniques to } \\
\text { study short term effects of the } \\
\text { strategically weighted vest. }\end{array}$ & $\begin{array}{l}\text { Completed for comprehensive } \\
\text { exam fall, } 2014 .\end{array}$ & 3 & IEEE, SH \\
\hline 6 & $\begin{array}{l}\text { Validate stride time and step length } \\
\text { metrics against the Vicon }{ }^{\circledast} \text { system. }\end{array}$ & $\begin{array}{l}\text { Completed for comprehensive } \\
\text { exam fall, } 2014\end{array}$ & 3 & IEEE, SH \\
\hline 7 & $\begin{array}{l}\text { Develop a computational methodology } \\
\text { to track long term trends in these } \\
\text { metrics and detect changes. }\end{array}$ & $\begin{array}{l}\text { Using OCPCM. Includes } \\
\text { clinical event prediction }\end{array}$ & 5 & $\mathrm{PH}$ \\
\hline 8 & $\begin{array}{l}\text { Use metrics and methodology in \#7 to } \\
\text { study long term effects of the } \\
\text { strategically weighted vest therapy }\end{array}$ & $\begin{array}{l}\text { Compared walk data } 30 \text { days } \\
\text { prior to, and } 30 \text { days following } \\
\text { therapy to identify changes. }\end{array}$ & 5 & $\mathrm{PH}$ \\
\hline 9 & $\begin{array}{l}\text { Use metrics and methodology in \#7 to } \\
\text { study impact of major interventions on } \\
\text { two or three subjects. Compare to } \\
\text { existing FRA instrument results. }\end{array}$ & $\begin{array}{l}\text { Presented correlation } \\
\text { between events generated as } \\
\text { part of } \# 7 \text { and FRA's for those } \\
\text { residents who had FRA's } \\
\text { taken. Additionally, looked at } \\
\text { change in metrics coincident } \\
\text { with the frequency falling of } \\
\text { resident } 3012\end{array}$ & 5,6 & $\mathrm{PH}$ \\
\hline 10 & $\begin{array}{l}\text { Use metrics and methodology in \#7 to } \\
\text { study long term change in metrics and } \\
\text { compare any changes to FRA's. }\end{array}$ & $\begin{array}{l}\text { First part completed along } \\
\text { with \#7. Compared results to } \\
\text { FRAs }\end{array}$ & 5,6 & $\mathrm{PH}$ \\
\hline 11 & $\begin{array}{l}\text { Determine the fraction of walks that are } \\
\text { not included using E. Stone's algorithm } \\
\text { due to occlusion which are considered } \\
\text { valid with the centroid based metrics. } \\
\text { Focus on Stride Time and Step Length. }\end{array}$ & $\begin{array}{l}\text { Completed in this dissertation. } \\
\text { Also included simulated } \\
\text { obstruction analysis. }\end{array}$ & 7 & None \\
\hline 12 & $\begin{array}{l}\text { Improve clustering algorithm from } \\
\text { currently implemented method (GMM } \\
\text { on } n+1 \text { clusters) to PCM and/or PFCM. }\end{array}$ & $\begin{array}{l}\text { Developed improved } \\
\text { clustering algorithm using } \\
\text { PFCM combined with PCA } \\
\text { transformation. }\end{array}$ & 4 & $\mathrm{PH}$ \\
\hline
\end{tabular}


Appendix II

Software Design Description Metrics Computation Component 


\section{Introduction}

\section{Purpose}

This software design document describes the architecture, design, and algorithms of the Metrics Computation Subsystem. It is written to be used and understood by anyone wanting to use this software in a larger application. It assumes a knowledge of MatLab.

\section{Scope}

This software system reads a list of centroid values for "Walk Data" (as opposed to "Path Data"). It will compute the various metrics and generate an output a single file for each resident.

\section{Background}

\section{Input Data Format}

The input data is stored, as of this writing, under the path "/mnt/mcp/xxxx/KinectData/WalkData" where "xxxx" is the resident ID for the walks in that apartment. In the folder WalkData, there is one folder for each day that there have been data collected. Each folder, in turn, contains a list of folders, each corresponding to one walk during the day. Each of these folders contains a set of raw image files which we do not use, and one file called "centList.txt" which stores the centroid data for that walk.

The file centList.txt has a list of centroid locations for each walk. The file has 8 tab separated columns. The first is a sequence number, the second is a time stamp for the record. The third through fifth are the $X, Y$, and $Z$ position of the centroid. Column 6 is the total height of the subject measured at the top of the head. The last two columns relate to the point cloud characteristics and are not used.

The records in the file are captured in reverse chronological order typically, though not required, at 15 records per second. 


\section{Walk Data}

\section{Entropy}

Entropy measures the uncertainty, irregularity, or randomness in a given signal. In this case, entropy will be used to measure the irregularity in the subject's gait. The hypothesis is that a healthy individual's gait is characterized by more regular, sinusoidal, movement laterally and vertically. As a subject's gait degrades due to age or disease, the gait can become more irregular. This would cause entropy measurements to increase as the subject's gait degrades indicating a higher risk of falling. Arafat et al. used entropy to study gait deficiencies and identify ataxia in horses [111]. In his work, he utilized three different computations of entropy. The first, equation 6, by Deluca and Termini in 1972 [112], is given by:

$$
H_{D T E}^{\delta_{k}}=-K \sum_{j} \delta_{k j} \log _{2} \delta_{k j}+\left(1-\delta_{k j}\right) \log _{2}\left(1-\delta_{k j}\right)
$$

Equation 7, developed by Pal in 1989 [113] is given here:

$$
H_{P P E}^{\delta_{k}}=K \sum_{j} \delta_{k j} e^{1-\delta_{k j}}+\left(1-\delta_{k j}\right) e^{\delta_{k j}}
$$

And equation 8, developed by Pal \& Bezdek in 1994 [114]:

$$
H_{\alpha Q E}^{\delta_{k}}=K \sum_{j} \delta_{k j}^{\alpha}+\left(1-\delta_{k j}^{\alpha}\right)^{\alpha}
$$

In all three formulae, $\delta_{k j}$ is the error signal in a particular direction. $\mathrm{K}$ is a normalization constant taken to be 1 in each of these measurements.

The entropy metrics determine the randomness or unpredictability of the subject's walks. In a healthy younger person, examining the movement of their centroid in the $\mathrm{Y}$ direction (lateral to the direction of motion) would show a fairly regular sinusoid as the centroid moves back and forth 
to keep the subject's center of mass above the planted foot. For a subject with difficulty balancing, as an example, there may be some additional random movement due to unsteadiness. It is this extra randomness that this metric will measure. For a regular, more sinusoidal-like signal, the entropy will be low. For a more irregular, random centroid path, the entropy will increase.

\section{System Overview}

This software will run on MatLab. It has been run successfully on MatLab versions 2010b, 2016b, and 2017a.

\section{Modules}

\section{ComputeEntropy.m}

This is the top level module for this system. When started, it initializes some flags and variables that control the overall function. The following table shows the initial flags and describes their functions.

\begin{tabular}{|l|l|l|}
\hline addABox & $\begin{array}{l}\text { If set to 1, creates a 24" x 24" box on the floor and, when } \\
\text { the subject's centroid is inside this box, adds 12" to the } \\
\text { centroid's Z value to simulate an occlusion in the } \\
\text { environment. }\end{array}$ \\
\hline useWalkData & $\begin{array}{l}\text { If set to 1, uses Walk data to generate the metrics. If set } \\
\text { to zero, the PathData is used instead. I have only used } \\
\text { PathData once in the last several years with poor results. } \\
\text { Use at your own risk. }\end{array}$ \\
\hline SamplesToSkip & $\begin{array}{l}\text { Allows you to only use every N } \\
\text { walk. }\end{array}$ \\
\hline skipSamples & $\begin{array}{l}\text { This is initialized to zero here. It is the counter used by } \\
\text { the previous "SamplesToSkip" to keep track of what has } \\
\text { been skpped. }\end{array}$ \\
\hline
\end{tabular}




\begin{tabular}{|l|l|l|}
\hline FPS & $\begin{array}{l}\text { Frames Per Second value used to compute the metrics. I } \\
\text { have been using } 15 \text { fps for all but the earliest analysis. }\end{array}$ \\
\hline newDataFormat & $\begin{array}{l}\text { This controls the data file and folder naming conventions } \\
\text { used to read in the data. If false, it reads in data using } \\
\text { expectations suitable for } 2011 \text { to } 2013 \text { data (with the IP } \\
\text { subject ID). This should probably be left at } 1 .\end{array}$ \\
\hline
\end{tabular}

The next "if" block determines which directory from which to start reading walk or path information. The string variable "source" defines the source of the walk data. Under this folder, there should be a single folder for each apartment. The variable "target" defines where the results file will be put.

As this file will process all resident apartments in the "source" folder, the next block will read in the directory list of apartments and loop through each. I will refer to this subsequently as the "I" loop. The lines with "strcmp" calls allow you to skip resident apartments.

The next set of lines opens up the output file. If we are creating a set with a simulated box, we will add the characters "-box" into the filename.

Next, a flag indicating that this is the first time through the loop is set. This flag controls the emission of a column header row with the names of the columns.

The path with the walk or path data for the residence is created next. This directory is scanned and all of the days for which there are walks is read into a list. This list is used in a second nested loop (the "J" loop) which processes each day.

Finally, the "K" loop is set up for reading each walk on a particular day. Entering this loop, you first see the logic around the "SamplesToSkip" variable set initially. If this is set to a positive integer, the loop will skip that many walks. I don't remember why I put this in, but I don't see the need for it anymore. Leaving this set as zero will keep any skipping from happening. 
Next, the timestamp from the filename is read. The old file format had the a prefix of 6 characters before the time stamp. The new file format does not. This logic simply selects the right one to extract the timestamp from the filename. This timestamp is then used to create an year/month/day/hour/minute/second timestamp using the "dateToDay" function and adding the number of seconds (as a fraction of a day) to the return value of the function.

Next, we prepare to read in the datafile. If we are using the old data format, the input file is stored at this level and named with the timestamp. If we are using the new data format, the input file is stored in a directory named with the time stamp. The name of the file with the centroid data is "centList.txt". With the correct name, the function "ReadMyData()" is called to read in the data from the disk file. The function returns a matrix with 5 columns (timestamp, centroidX, centroidY, centroidZ, and total height) with one row for every step. The function also adjusts the input data to the passed Frames Per Second value (FPS) by culling extra data or interpolating missing data. The details will be provided in the documentation for that function.

At this point, we are ready to start computing metrics. The function "StretchOutWalks6" is called passing it the $X, Y, Z$, and Height data (ZP). Also, the frames per second and the number of frames in the estimation window are also passed. The second to last parameter defines the threshold to be computed with "walk" or "rest" measurements. The idea at the time, particularly with "path" data, was to separate purposeful walks (> $5 \mathrm{in} / \mathrm{s}$ ) with very slow movements or even resting. The last parameter is not used and I cannot remember why it was put there.

The following values are returned by the function:

\begin{tabular}{|l|l|}
\hline xeMove & X error vector while the subject is moving $>$ than the threshold passed. \\
\hline yeMove & Y error vector while the subject is moving $>$ than the threshold passed. \\
\hline
\end{tabular}




\begin{tabular}{|l|l|}
\hline zeMove & Z error vector while the subject is moving $>$ than the threshold passed. \\
\hline xyeMove & XY error vector while the subject is moving $>$ than the threshold passed. \\
\hline xeRest & X error vector while the subject is moving $<$ than the threshold passed. \\
\hline yeRest & Y error vector while the subject is moving $<$ than the threshold passed. \\
\hline zeRest & Z error vector while the subject is moving $<$ than the threshold passed. \\
\hline xyeRest & XY error vector while the subject is moving $<$ than the threshold passed. \\
\hline iSpeed & $\begin{array}{l}\text { Vector containing the instantaneous speed between the current and } \\
\text { previous point. }\end{array}$ \\
\hline Speed & Vector containing the speed at that point expressed in MPH. \\
\hline Asymmetry X, Y, & $\begin{array}{l}\text { Three values containing the asymmetry values in the X, Y, and Z } \\
\text { directions. }\end{array}$ \\
\hline $\begin{array}{l}\text { Peak to Peak X, Y, } \\
\text { Z }\end{array}$ & $\begin{array}{l}\text { Three values containing the Peak to Peak metrics for the X, Y, and Z } \\
\text { directions. }\end{array}$ \\
\hline tenFootWalk1 & $\begin{array}{l}\text { Time spent on the walk adjusted for a 10 foot walk. This one uses } \\
\text { estimated distance. }\end{array}$ \\
\hline tenFootWalk2 & $\begin{array}{l}\text { Time spent on the walk adjusted for a 10 foot walk. This one uses true } \\
\text { distance. }\end{array}$ \\
\hline tDist & $\begin{array}{l}\text { Estimated Distance / True Distance. } \\
\text { Estimated distance }- \text { length of the expected distances }\end{array}$ \\
\hline walkTime & $\begin{array}{l}\text { The distance }- \text { length of the actual path of the resident. } \\
\text { debugging }\end{array}$ \\
\hline pentAtRest & Percentage of frames classified as at rest (speed $<$ passed threshold) \\
\hline failureFlags &
\end{tabular}

Average speed is computed next by simply taking the mean of the returned speed vector. Next, StrideTime is computed using the "DeduceStrideLengthAndSway5()" function. Passed the $Y$ and Z motion vectors, FPS value, and the minimum and maximum entropy values, this function returns the stride time and three flags indicating failures to deduce stride time. 
At this point, we check if we have managed to compute a stride time value. If we have not, we cut the minimum entropy value in half and retry. This is repeated a few more times. The highest minimum entropy value is used to compute stride time. The code in the else statements for each check that deals with "timeList" and "multipliers", I don't think, has any effect. If memory serves, the original purpose of this was to do a weighted average of several ST computations, but this does not seem to be doing this at all. In the near future, I may remove this code and see what happens. But until then, it is still there and I doubt it does anything useful.

Once StrideTime is computed, it is passed into "FindBounceAndSway()" which, as the name suggests, finds bounce, and sway. It also finds stepRatio, and left and right step times. Left and Right step lengths are computed using left and right step times and speed. Next, left step length (which should more properly be called StrideLength) is computed by adding the left and right step lengths. Step Time, which is properly named, is computed as the average of the left and right step times. Average Speed is computed as the average of the speed vector. CHeight (centroid height) is the average of the $\mathrm{Z}$ vector, and theight (Total Height) is the mean of the total height vector.

The next block of 12 lines computes the various entropy metrics. The first four lines compute the entropy values based upon $X, Y, Z$, and Speed for the "moving" portion of the walks. The "AS" entropy metric is calculated next and is based upon the instantaneous speed vector while the "Speed" entropy measures are based upon the speed vector. Next the $X, Y$, and Z Resting entropy values are computed from those vectors. Lastly, the Total Height and $X Y$, and $X Y$ at rest entropy values are computed. 
The last metrics computed are the Average Entropy values which are the simple averages of the three entropy metrics for each metric.

Finally, there is logic to write column headers to the output file if it is the first time through the loop. The metrics are assembled into matrix of all walks in that particular day and, when the entire day is processed, the matrix is written to disk in CSV format. The last metric in the matrix is a dummy value and is there simply to facilitate writing a carriage return at the end of the line.

\section{dateToDay.m}

This function takes a date (month, day, and year) and converts it to the number of days from January 1,2000 . It uses a lookup table to find the total days passed in the year at the first day of the month, adds the number of days in the month. Lastly, it adds the year while adjusting for leap year.

\section{DeduceStrideLengthAndSway.m}

The data is first filtered to remove some of the high frequency components. Next, the entropy of the $\mathrm{Z}$ signal is computed. If the resulting Deluca-Termini entropy value is in the range [1..10], the signal is suitable for finding stride time. If not, set StrideTime to NaN and return.

Once the entropy meets the requirements, a FFT of the $Z$ signal is computed. The first point is zeroed out (the highest peak) and the remaining highest peak is identified. This is then converted into a period which is the $1 / 2$ of the stride time. The result is doubled yielding the stride time. 


\section{GenerateFuzzyVector2.m}

First, the probabilistic vector is computed. The denominator is computed as the first norm of the input vector. The numerator is the original vector squared. The probabilistic vector is formed by dividing the numerator by the denominator. Next, the minimum, maximum and range for the input vector is computed and used to normalize the input vector squared. Any values $==0$ or 1 are replaced with 0.00001 and 0.99999 to keep the algorithm from running into problems with the edges.

Finally, the entropy of each element is computed using the formula for each method. Lastly, the elements are summed as appropriate for the entropy method.

isEven.m

This function simply returns true if the passed number is even by comparing the modulus 2 result to zero.

\section{ReadMyData.m}

This function reads the centroid data from the passed source file. It returns the centroid data adjusted to the passed sample rate (typically 15 frames per second) and a count of the number of frames that were blocked by a simulated obstruction. The "textscan" MatLab function is used to load the disk file into memory and parse based upon the provided format string. Each column is then isolated and a set of time stamps, relative to the first frame, is computed for each frame. Next the data is adjusted to the passed frame rate by either interpolating missing points or culling extra points. To do this, a "fixedData" matrix is created which will hold the adjusted data. For each point in this new matrix, minimum and maximum times corresponding to each point in the output data set are computed. The subset of walks 
between the minimum and maximum times for each point are extracted. If there is one point, it is used with no modifications. If there are multiple points extracted, an average is used. If there are no points, a NaN is inserted as a placeholder indicating missing data.

The output matrix is then scanned looking for NaN data. If one is found, it is replaced by the average value of the closest two sets of centroids, one on each side of the missing point.

Finally, if we are simulating an obstruction in the center of the floor, each centroid is checked to determine if it is within the simulated obstruction. If it is, 12 inches are added to the centroid value.

\section{StretchOutWalk6.m}

In addition to converting the room coordinate centroid values into error values, as described in dissertation chapter 3 , this function directly computes most of the basic metrics including asymmetry, peak to peak, average speed, and walk efficiency. It is passed the $X, Y, Z$, and Total Height which are generated by the previous function, a window size to compute best fit lines, and an "at rest" speed.

The function loops through all points from $\times(1)+$ windowSize/2 to $\times(e n d)-$ windowSize/2. First, a best fit line for the window size number of points centered on the current point is computed. Next, the average speed over the window is computed by using the Pythagorean theorem to compute the distance and dividing by the window size. Instantaneous speed is also computed by looking at the distance between the current point and the previous point also divided by the window size. Next, the "atRest" flag is computed. This flag controls the placement of this point into the "moving" or "at rest" matrices. As the "at rest" data is not used, this effectively culls "at rest" data. 
Data is considered "at rest" if the average instantaneous speed over half the passed window is less than the passed "at rest" speed. Once the flag switches state, data for the last half window of time is copied from one array to the other.

Asymmetry is computed next by dividing the mean of each direction by the largest absolute value of all points in the array. Peak to Peak is also computed in each direction by computing the absolute value of the difference between the maximum value and minimum values. Finally, walk times and walk efficiency are computed. 
Appendix III

Software Design Description Vicon ${ }^{\circledR}$ Validation Component 


\section{Purpose}

This SDD describes the Vicon Validation component which is used to validate the functions StretchOutWalk6.m, DeduceStrideLengthAndSway5.m, and FindBounceAndSway.m from the Compute Metrics Component.

\section{Scope}

This software system reads a set of centroid positions, stride time, and stride length data from a data file generated using the Vicon ${ }^{\circledR}$ system. The code uses these data to verify the proper function of the three functions mentioned in the above paragraph.

\section{Background}

This simple component computes the stride length and stride time metrics using the same functions as described in the Compute Metrics Component SDD document and compares it against the same values generated by the Vicon ${ }^{\circledR}$ Gait Analysis System. Error values and probability density functions are computed to compare results.

\section{Foo.m}

The top level file is called "foo.m". It starts by loading a file containing Vicon ${ }^{\circledR}$ ground truth data for a number of different walks for a number of different subjects. Each walk also has two different Kinect camera views (different cameras) and two different filter algorithms. For each individual walk, the data file already contains the stride time, stride length, and average speed values for each walk and the centroid positions for each walk.

The function starts with four "for" loops for each resident, walk, camera and filter. For each walk, the frame rate is adjusted to 15 FPS using the FixFrameRate function which, uses an improved implementation of the same approach used in ReadMyData.m (Appendix II). The functions StretchOutWalk6.m and DeduceStrideLengthAndSway5.m are called to compute the 
error vectors and estimate stride time. FindBounceAndSway.m is called next to compute left step time, right step time, left step length, and right step length. StrideLength is computed as the sum of the left step length and the right step length.

Finally, error vectors are computed for stride time and stride length by subtracting the ground truth from the calculated values. Probability density function plots are generated using MatLab's gkdeb() function. The PDF's along with error values and other statistics are displayed.

\section{FixFrameRate.m}

While the code to fix the frame rate to a passed value in this module uses the same algorithm as in the ComputeMetrics component, its implementation reflects an improved understanding of MatLab and, consequently, is written more efficiently.

\section{FindBounceAndSway.m}

As this is one of the functions being validated, this is a direct copy of the file in the ComputeMetrics component. For the results to be meaningful, this must be a direct copy.

\section{StretchOutWalk6.m}

As this is one of the functions being validated, this is a direct copy of the file in the ComputeMetrics component. For the results to be meaningful, this must be a direct copy.

\section{DeduceStrideLengthAndSway5.m}

As this is one of the functions being validated, this is a direct copy of the file in the ComputeMetrics component. For the results to be meaningful, this must be a direct copy. 
Appendix IV - SDD for Resident Validation Component

\section{Software Design Description Resident Validation Component}




\section{Purpose}

This SDD describes the Resident Validation component which is used to validate the functions StretchOutWalk6.m, DeduceStrideLengthAndSway5.m, and FindBounceAndSway.m from the Compute Metrics Component.

\section{Scope}

This software system reads standard resident data and computes stride time, stride length, and average speed using both centroid data as well as Erik Stone's approach (the "existing method"). The code uses these data to verify the proper function of the three functions mentioned in the above paragraph.

\section{Background}

This simple component computes the stride length and stride time metrics using the same functions as described in the Compute Metrics Component SDD document and compares it against the same values generated by the Vicon ${ }^{\circledR}$ Gait Analysis System. Error values and probability density functions are computed to compare results.

\section{RunStuff.m}

This function takes, as a parameter, the id of a resident. If this is not specified, the value is assigned in this function. Doing this allows it to be run as part of look which runs through all residents, or runs for a single resident. The first part of this function, (down to the commented line "ADDED BY BW") along with the functions it calls, were originally provided by Erik Stone to compute stride time, stride length and average speed using his algorithms. This is done with the functions "openKinectWalkFiles()", "openKinectCCTSFiles()", and "calcStrideParamsKinect()". Following the last call, the cell arrays "strideParams" contains the results. 
Next, for every walk that the existing method was able to compute values

(strideParams $\{r, c\}(1)==3$ ), the centroid based stride time, stride length, and average speed are computed using StretchOutWalk6.m, DeduceStrideLengthAndSway5.m, and

FindBounceAndSway.m. Outliers are removed using the "IdentifyOutliers()" function. Similarly to how the Vicon ${ }^{\circledR}$ Validation results are shown, PDF's are computed for each metric and disparity values from the existing method. 
Appendix V

SDD for Improved Clustering

Component 


\section{Motivation}

The walk data collected for each residence captures all walks in the room. This includes walks by the resident, walks by guests, and walks by facility staff. In order to properly identify the person responsible for each walk in order to allow individual monitoring, this collection of walks must be separated by person.

Initially, a Gaussian Mixture Model was used to cluster the data. For residents with distinct differences in one or more features, this approach works well. However, in two of the apartments, the GMM approach was insufficient to separate residents. Consequently, a new approach was developed.

\section{Methodology}

This component operates on the computed metrics. The approach used separates these walks into chunks of walks done within 14 day periods. The number of clusters expected is fixed at the number of residents per apartment. If PCA transformation is desired, the PCA transform matrix is calculated and the minimum number of columns corresponding to $95 \%$ of the variance in the data are selected. The transform matrix is then applied to the input data.

Next, the optimal set of features to cluster the data is selected. This is done by simply clustering all 16 combinations of four candidate features (Trunk Sway, Step Ratio, Step Length, Average Entropy XY). These candidate features are combined with the required features (Step Time, Total height, Avg. Speed, Avg. Instantaneous Speed) to create the input feature set for the clustering algorithm. After each combination is clustered, the Davies-Bouldin index is computed on each combination and the combination with the highest D.B. index is selected as the winner.

The best set of features is then clustered using the PFCM algorithm (alternatively, the KMeans algorithm can be selected, though the results obtained using this method are 
significantly inferior). Once the labels have been returned, the entire dataset is separated into clusters based upon the labels. As the label numbers can vary based upon the starting conditions of the data, labels are assigned by choosing the existing clusters (from previous chunks) for which the cluster centers are closest to the new chunk. In other words, if the center of resident \#1 is closer to the center of cluster \#2 for the current chunk, cluster \#2 is assigned to resident \#1, and cluster \#1 is assigned to resident \#2. After being assigned, all data $>2$ standard deviations away from the center of each cluster are culled. Finally, each cluster is saved individually.

\section{Modules}

\section{ChunkClusterPFCM.m}

This module is the top level module that actually does the clustering. When called, it initializes the global variables and sets the code to use PFCM clustering (Clustering = ' $\mathrm{P}$ ') and use PCA transformation (PCA=1). Next the required and scanned features are defined. The data is cleaned up using "CleanUpData", then we start the clustering itself.

UpdateChunkBoundaries is called to set the minimum and maximum time stamp associated with the current cluster. Next, the "EstimateNumClusters" function is called and the estimated number of clusters is set to 1 more than this value. The function

"ScanForBestFeatures" is called to select the best features for the cluster.

The selected set of best features for this chunk returned and the PCA transform is generated. The MatLab PCA function generates a vector containing the variance explained by each component. This vector is used to select the minimum set of components that explain at least $95 \%$ of the variance. 
Lastly, the function "CleanAndAssignClusters" is called to remove outliers and properly assign clusters from this chunk to the entire set of clusters for this dataset. After all this is done, each cluster is saved into a separate datafile.

\section{CleanAndAssignClusters.m}

This module is passed the clustering results for a particular chunk, culls any walk that is more than 2 standard deviations from the cluster center, and assigns each chunk cluster to the proper cluster in the accumulated results.

As no logic is contained here to deal with 3 or more residents in an apartment, a check is made to ensure that this is not the case.

Cluster \#1 is selected and its data isolated from the full set. The mean and standard deviation of the distance of each walk to the cluster center is computed and all walks that are > 2 standard deviations away from the cluster center are removed. If this is a single resident apartment, the cluster is stored and the module returns. If there is a second resident, the same process is repeated for the second cluster.

Lastly, the clustered chunks are assigned to their proper resident. If this is the first chunk, the largest cluster is assigned to the resident \#1 and the second cluster is assigned to resident \#2. If this is not the first chunk, the center of each chunk cluster is compared to the centers for the first chunk clusters and each chunk cluster is associated with the proper resident based upon distance between the chunk cluster center the resident cluster center.

\section{CleanUpData.m}

This module is called early on to clean up data prior to clustering. The first thing it does is to sort walks based upon time of occurrence. Next it removes all walks that contain NaN for 
any metric. Finally, it culls all walks for which at least one feature lies outside $+/-2$ Standard Deviations away from the mean of the feature.

\section{ClusterData.m}

This is the top level function that works on one apartment. After loading the metrics file, some control variables are set. These are:

\begin{tabular}{|l|l|}
\hline Variable & Meaning \\
\hline nClusters & $\begin{array}{l}\text { A Nx2 matrix of resident apartments and the number of clusters expected in } \\
\text { that apartment. Number of clusters is equal to 1 + number of residents. } \\
\text { This variable is no longer used. }\end{array}$ \\
\hline useTallerPerson & For nClusters > 2, the cluster with the taller height is selected when needed. \\
\hline chunkSize & Size of clustering chunk (in days). \\
\hline dayStep & $\begin{array}{l}\text { Number of days advanced between clusters (in days - typically matches } \\
\text { chunkSize) }\end{array}$ \\
\hline
\end{tabular}

After the control variables are set, a "test" variable is checked and, if set, the code substitutes test data for the read data. The last pre-processing step marks walks as having been done wearing the weighted vest. This allows an output file set to be created with no vest walks included.

Clustering is actually done by the function ChunkClusterPFCM.m (described earlier). If the apartment has a resident participating in the weighted vest study, two different sets of output files are created - one including any walks while the vest is being worn, and one with vest walks excluded. It should be noted that at this point, it is not possible to programmatically identify which resident is undergoing weighted vest therapy, so for two resident apartments 
with one resident wearing the vest, all walks by BOTH residents while the vest is being worn are removed.

\section{ComputeDaviesBouldin.m}

The Davies-Bouldin index is an internal cluster validity measurement the relates the sum of the scatter for each cluster to the distance between each cluster. The idea is that two clusters that minimal scatter and maximal separation are the best pair. The algorithm here is one that I had used for an unsupervised learning assignment and pre-dates my knowledge of the existence of a MatLab version.

\section{EstimateNumClusters.m}

This function estimates the number of clusters based upon the data. For the residences where the number of residents are know a priori, that value is simply returned. For datasets where the number of residents are not known, an estimate is made by using PFCM clustering to cluster into 2, 3, and 4 clusters. The number of clusters that yielded the lowest Davies-Bouldin score is selected as the number of residents in the apartment.

\section{ExtractFullSet.m}

This module traverses the set of results for each chunk and assembles each chunk into the full dataset for each resident. This is then saved and used in other analysis modules.

isBitSet.m

This function returns TRUE if bit number " $b$ " (counting from zero) is set in passed numeric value " $n$ ". 


\section{MakeTimeStamp.m}

This utility function takes an array of date components and time expressed as a fraction of a day since the beginning of the day and computes a single floating point representation of the date/time as the number of days since January 1, 2000.

\section{MarkVestWalks.m}

This utility function marks all walks that occur while the weighted vest is worn in the apartment. The event on and event off times are loaded and all walks between the time the vest is donned and the next time the vest is doffed are marked.

\section{myDist.m}

A highly simplified and faster implementation of MatLab's general purpose pdist2() function.

PFCM.m

This is a direct implementation of the PFCM algorithm from [119] developed, tested, and used in Unsupervised Learning.

\section{ScanForBestFeatures.m}

This function selects the set of features that yield the best Davies-Bouldin index. A For loop is started from zero to $2^{\text {nFeatures }}$. For 4 required features and 4 optional features, this makes 16 combinations. If the normalize flag is true, the data is normalized between $[0,1]$. The required features are then copied into the PFCM input data array, followed by the scanned features being used for this pass.

The PFCM clustering algorithm is then used to cluster the results and the Davies-Bouldin index is computed and stored. Following all 16 passes, the set of features that yielded the 
lowest Davies-Bouldin index is selected as the optimal set of features. A matrix containing all walks with the optimal set of features is returned.

UpdateChunkBoundaries.m

This utility function updates the start and end indicies for each chunk based upon the number of days per chunk (typically 14) and the dateStep value (typically the same as the chunk size). 
Appendix VI

SDD for Short Term Analysis 


\section{Motivation}

The purpose of this component is to examine the effects of a particular therapy, in this case the strategically weighted vest therapy, on a resident's metrics. Initially, an attempt was made to examine individual walks but it quickly became clear that aggregating the walks and examining general behavior around a collection of events would yield results.

\section{Methodology}

The horizontal axis of the plot is broken into a specified number of bins representing a portion of the specified time window. Next, each walk is aligned to the corresponding bin relative to each vest on and vest off event. The value of each metric is summed in its bin. While unlikely, a walk may be recorded in multiple bins if it is within the window for two or more events (window size of 3 hours, and resident wore the vest for 1 hour). After all walks have been included, the sum in each bin is divided by the number of values added to the bin - the average value of all of the walks included in the bin.

Next, the Minimum Detectable Change is computed for the left side (before the event) of the graph. The mean of the right side of the graph is also computed and compared to the $M D C$ value. If the mean on the right side of the graph is greater than the left hand mean + $1 M D C$, or less than the left hand mean - 1MDC, the change is flagged as significant.

\section{Modules}

\section{Analyze.m}

This is the main script for this analysis component. First, some variables are set up for the analysis. Important ones to set are shown in table A6.1. The most important ones are the variables that start with "binEvent...". These control the bin width and size of the resulting plot. AverageAllEvents controls the aggregation of all of the walks around a particular type of event. This should be left at 1. "binEventWindow..." sets the number of days, hours, minutes, and 
seconds that will be shown in the plot. "binEventNumberBins" sets the number of bins in the plot. The last two in this section, "binMultipleRunStep" and "binNumTotalPasses" are used to scan for the optimal plot parameters to maximize the significant changes in the number of metrics.

The next set of settings controls data smoothing. This is left in but is no longer used.

The code enters a loop for the number of passes to make - for the basic plots, this just runs once. After loading data files, the event times are turned into compatible timestamps. The data is then sorted by timestamp (it should already be sorted but is here to increase robustness). If the number of people in the data set is $>1$, the data is clustered using a Gaussian Mixture Model, as described in "PruneAndCluster3()".

Next, each metric is considered in a loop. If trend lines are desired, those are generated first. Next, if we are going to compute daily averages, those are done next. No plots are generated for this section, but the statistics are printed to the console.

The short term plots are generated next. The "binEventWindow..." values are converted into a decimal number of days. The EventBin() is called to create and display the graphs. If plots of the changes over time are selected, those are generated next. If averages by day are selected (currently set to "off"), those are computed next. Lastly, a set of curves with confidence intervals are plotted if selected (currently, they are not).

Finally, if multiple passes are selected, the post processing is done here and the best window size is selected based upon the number of metrics that show significant changes.

\section{ComputeFowlkesMallows.m}

This function takes a series of labels from many clustering attempts and selects the best set of labels based upon the Fowlkes Mallows index for each combination of labels. The 
function is passed a set of labels for multiple runs (each column is a run). The parameter " $\mathrm{n}$ " is not used and is explicitly set upon entry into the function. To reduce the computational load, as each set of labels could have as many as 60,000 values, the rows for the entire set of labels are randomized and $5 \%$ of these rows are selected for analysis. Note that rows are randomized, with no changes to the data in each row. This is important as not ensuring this would invalidate the usefulness of Fowlkes Mallows computations. Once the NxN matrix ( $N$ is the number of clustering attempts) of Fowlkes-Mallows scores are computed, the highest score is selected. The row and column indices for this value correspond to the two clustering sets that agreed the closest. The row index is then chosen as the best set and returned to the caller.

\section{EventBin.m}

This function computes and plots the short term analysis plots. The bin size is computed based upon the number of bins and the desired amount of time to include in the plots, both before and after the event. Once the setup is finished, the function uses a double set of loopsthe outer loop runs for every recorded event, and the inner loop runs for each data point. The loop computes the bin that the point would be in based upon it's time relative to the event. If the bin number is $<0$ or $>$ the maximum number of bins, the point is dropped. Otherwise, it is added to the bin count for the bin, and the number of data points added into the bin is also incremented. When finished, the average value for each bin is computed by dividing the sum by the number of points.

Next the MDC value is computed based upon the data points before and after the event. This MDC value is then used throughout to look at both vest on and vest off changes. Next, outliers are removed from the data set. An outlier is defined as a data point greater than 2 standard deviations from the mean. Finally, two plots are made - the binned points around the 
vest off and the vest on event. Note that the data is aggregated as there was insufficient data for each vest event to look at it individually.

\section{FindMDC.m}

This function computes the Minimum Detectable Change value for the passed data. The computation is a two-step process using the following formulae. The first step is to compute the statistical " $\mathrm{t}$ " value using the following:

$$
\mathrm{t}=\frac{\overline{\mathrm{X}}_{\text {pre }}-\overline{\mathrm{X}}_{\text {post }}}{\mathrm{S}_{\mathrm{X}_{\text {pre }} \mathrm{X}_{\text {post }}} \sqrt{\frac{1}{\mathrm{n}_{\text {pre }}}+\frac{1}{\mathrm{n}_{\text {post }}}}}
$$

Where

$$
S_{X_{\text {pre }} \mathrm{X}_{\text {post }}}=\sqrt{\frac{\left(\mathrm{n}_{\text {pre }}-1\right) S_{\mathrm{x}_{\text {pre }}}^{2}+\left(\mathrm{n}_{\text {post }}-1\right) S_{\mathrm{X}_{\text {post }}}^{2}}{\mathrm{n}_{\text {pre }}+\mathrm{n}_{\text {post }}-2}}
$$

and $\bar{X}_{\text {pre }}$ and $\overline{\mathrm{X}}_{\text {post }}$ are the means for the pre-event distribution and the post-event distribution respectively. $\mathrm{n}_{\text {pre }}$ and $\mathrm{n}_{\text {post }}$ are the number of samples in the pre- and post-event distributions. $\mathrm{S}_{\mathrm{X}_{\mathrm{pre}}}^{2}$ and $\mathrm{S}_{\mathrm{X}_{\mathrm{post}}}^{2}$ are the variances for each distribution. Finally, MDC is computed using the following formula:

$$
M D C=t \sqrt{\frac{M S E}{n_{p r e}}+\frac{M S E}{n_{\text {post }}}}
$$

where MSE is the pooled mean squared error which is estimated by the variance for the pre-event distribution.

\section{GenerateTrendLines.m}

This function generates trend lines on the passed data. The first parameter is the data to be analyzed. The second parameter specifies the number of trend lines to generate. If this value is equal to two, the pivot parameter specifies the point shared by each of the two trend lines. If more than two, the lines are computed equally spaced. 


\section{MakeTimeStamp.m}

This is the same function as described in the Improved Clustering Module.

\section{PlotOverTime.m}

This function averages the metrics over each day returning a single matrix with each feature over the second dimension and each day along the first dimension. It is passed the set of timestamps (real number corresponding to number of days (including fractional days) since January 1, 2000 and midnight). The function first identifies the first and last day by using the floor of the timestamp. Next, it steps through each day and creates a subset of all metrics for that day. This subset is then averaged and added to the output array. The second "for" loop identifies all rows with missing data (timestamp and data $==0$ ) and removes them. This will create an output matrix with fewer rows than days if not all days are represented in the data. Note that the "slope" output is not used and set to zero.

\section{PruneAndCluster3.m}

This function clusters the input data using a Gaussian Mixture Model. It loops through a set number of iterations (currently 50) and clusters the data each time. The resulting labels are saved for each time and is passed into the ComputeFowlkesMallows() function where the best set of labels is selected. These labels are then re-labeled such that the largest set is labeled as " 1 ", the next largest as " 2 ", and the smallest is " 3 ". For single resident apartments, one " 1 " and "2" are used.

\section{PointFilter.m}

This function filters the input data by simply averaging the passed number of points centered on the current point for each point in the dataset. 


\section{Appendix VII - SDD for Long Term Analysis}

Note: the code for this module is stored in the folder "EventAnalysis".

\section{Motivation}

This module analyzes the data to answer two distinct questions. First, which metrics change coincidently with any of the resident's clinical events. Secondly, the module determines if there are any significant changes in the metrics over two, non contiguous, periods of time. Lastly, additional code has been added to study the behavior of the metrics for resident \#2.

\section{Methodology}

The approach for this module is to compute a mean, standard deviation, and MDC over the period of data leading up to the event, and comparing it to the same amount of data following the event. The "before" and "after" blocks of data can either be contiguous, or disjointed. The events used are any of the clinical events in the resident's medical record, as well as any vest on and vest off events.

As metrics may change in such a way that the mean may not change, but the statistical distribution of the data may change, the Kolmogorov-Smirnov test was also used to evaluate the null hypothesis that the metric's data before and after the event are similar.

\section{Modules}

\section{CompareMeanShift.m}

\section{CompareMeanShiftSplit.m}

Both functions are similar with the major difference being the first function looks at contiguous data around a single event. The second function looks at the data for a period of time before the first event and compares it to the period of time after the second event. 
The first function is used for specific events which refer to a single point in time. Fall events, E.D. visits, individual vest events, and hospitalizations fall under this category. The second function is used when the "event" of interest occurs over a long period of time. In this study, the "event" is weighted vest therapy and the "first" event is the first time the resident dons the vest. The second event is the last time the resident doffs the vest.

The data which occurs within the passed window before the event is isolated and basic statistics are computed. Next the data occurring after the event (or after the second event if using the second function) is isolated and statistics are computed for it as well. The MDC is computed for the first range and is compared to the change (if any) computed for the second set of data. If the data following the event indicates a significant change (> 1MDC), this is logged as a significant change. Additionally, the K.S. test is performed on the before and after data. The change is considered significant if the K.S. test rejects the null hypothesis at a confidence of $95 \%$.

\section{ComputeAggregate.m}

This utility function combines the metrics which make up the aggregate metrics. The individual metrics are normalized in the range $[0,1]$ and those metrics which show improvement by increasing are subtracted from 1 . This ensures that all metrics show improvement by decreasing. 


\section{Appendix VIII - SDD for Predictive Analysis Component}

NOTE: The code for this module is stored in the directory LongTermAnalysis.

\section{Motivation}

This component performs the predictive analysis as described in chapter 6 . The goal is to monitor the long term trends in various metrics and identify any outliers which may indicate a sudden change in the resident's physical condition and may portend a fall or other clinical event.

\section{Methodologies}

Using the PCA/PFCM clustered data as input, this component will use the One Class PCM algorithm to identify outliers in the data. These outliers are the possible harbingers of clinical events. These are then scored against the ground truth consisting of all falls, hospitalizations, and ER Visits by the resident over the study period.

\section{Modules}

\section{AlignDataToFRA.m}

This function creates a matrix of the average value of each metric over each "window" day period centered on each Fall Risk Assessment. For each date in the FRA dataset, all of the walks are extracted that occur in the range [date-window, date+window] where date is the date the FRA was performed and widow is a passed parameter indicating the size of the average. In this research, the value of 1 is used. Two matrices are returned, the metrics matrix has one row for each FRA and the average of each metric. The fraData has the FRA data for the 6 instruments on each day FRA studies were done. 


\section{CheckCorrelation.m}

This function computes the Pearson correlation using MatLab's corrcoef() function between each combination of FRA and Metrics. For the metrics, the entire passed set is used. For the FRA values, columns 28 to 33 in the datafile are used as those correspond to the final value of each of the 6 FRAs used.

Also, a quick indication of the long term behavior of the metrics as needed so the average value of each metric over the first five percent of the walks is compared to the average value over the last $5 \%$ of the walks. This is displayed along with the correlation results.

\section{dateToDay.m}

This utility function converts the passed day, month, and year to the number of days since January 1, 2000 adjusting for leap years.

\section{FindBestSet.m}

This function finds the best set of metrics to identify outliers using the clinical event history to score the results. FullSet is the full dataset to start with. FullSetldx is the column numbers for each feature in the FullSet matrix. NNLabels are the clinical events, Score and FP are the best scores and false positive scores so far respectively.

After initializing global data, each feature is removed one-at-a-time and replaced when done. For a matrix of $\mathrm{N}$ walks of 16 features, this look will run 16 times with each unique set of 15 features. If the "UsePCA" flag is set (typically it is NOT), a PCA transform is done on the data prior to being clustered. LabelOutliers is called to identify outliers and the output of this function is scored. This is repeated for the remaining combinations of metrics. The best pair of scores (maximize TruePositive, then minimize false positive) is identified and the corresponding set of features is isolated. 
This best feature set is then passed into a recursive call of this module in order to determine if any features in this set can be removed to improve results. This recursion is repeated until an improved score is not obtained or only one feature is left. The final, best set, of features (or individual feature) is passed back along with the score. Each instance of this module that recursively called itself compares the returned score to it's best score. If the returned score is better, the returned results are passed upwards. If the current instance's results are best, those are passed back.

\section{FindSlopes.m}

This function computes the change in value between each pair of peaks and valleys or valleys and peaks. This is similar to the slope, but does not include the " $X$ " component of the slope.

\section{IndexOfDay.m}

This utility function returns the lowest index in the metrics matrix for which the walk occurs on the passed date.

\section{LabelOutliers.m}

This function uses the OCPCM function to label outliers in the input data set. After initializing some variables, the OCPCM function is run on the entire data set. A center and vector containing possibilities for each walk are returned. The walk with the lowest possibility of membership is removed and the process is repeated. For all but the first time, the movement of the center of the cluster between the current and previous loop is computed.

This movement of the center of the cluster should be very small as the first few points are removed. As more points are removed which are more likely to be in the cluster, the distance moved by the cluster center should increase until walks which are not outliers start to 
be removed. This corresponds to a flattening of the difference vs. iteration curve. It is at this point where walks that are highly likely to be part of the cluster are being removed.

This knee is identified next. Starting at point 50, the slope of the one hundred and one points centered on this point are computed. This is done for each point for which a difference is computed up to the $\mathrm{N}-50^{\text {th }}$ point. The first minimum slope (including negative) is identified. This value is most likely the point where the curve turns flat indicating the knee of the curve. The possibility value at this point is determined and a threshold of $75 \%$ of this value is determined. Finally, all possibility values less than this threshold are identified as outliers.

\section{LoadFRAData.m}

This utility function loads the data from the FRA results. As all the FRA data for all residents are in the same file, this function will parse the data file and pull out those data records belonging to the desired resident.

\section{OneClassPCM2.m}

This is an implementation of the OneClassPCM algorithm developed by the University of Missouri Sensor Team and described in [93]. The original file OneClassPCM.m contains the direct implementation of the cost function described in Wang's dissertation. This file is an optimized version that will execute more quickly.

\section{ParseEventFile.m}

This function reads in the contents of the clinical and vest event file for all residents and extracts the events for a particular resident. Each event is coded by its type and the return value is a matrix with time stamps and event codes. 


\section{ScanForEvents8.m}

This function is an older method of identifying events and is no longer used.

Score.m

This function scores the results of outlier detection. The function is passed the events as ground truth, an array of predictions, one element per day, with values of 0 if there is no event that day, and 1 if there is an event, and the number of days (n) to use as a window both before and after the event. The predictions matrix is scanned, one by one, and each score is incremented as described here:

\begin{tabular}{|l|l|l|}
\hline Classified as & If prediction(i) is & And \\
\hline True Positive & $\begin{array}{l}\text { Prediction }==1 \text { and } \\
\text { sum(events(i:i+n) })>0\end{array}$ & $\begin{array}{l}\text { All predictions }[\mathrm{i}+1, \mathrm{i}+\mathrm{n}] \text { are } \\
\text { ignored. (set to zero) }\end{array}$ \\
\hline False Positive & $\begin{array}{l}\text { Prediction }==1 \text { and } \\
\text { Sum(events( }(\mathrm{i}: \mathrm{i}+\mathrm{n}))=0\end{array}$ & Nothing. \\
\hline False Negative & $\begin{array}{l}\text { Events(i)==1 and } \\
\text { Sum(predictions }(\mathrm{i}: \mathrm{i}+\mathrm{n}))=0\end{array}$ & Nothing. \\
\hline True Negative & All others & Nothing. \\
\hline Changes & $\begin{array}{l}\text { Events(i) }==1 \text { and } \\
\text { Sum(predictions }(\mathrm{i}: \mathrm{i}+\mathrm{n}))=0\end{array}$ & Nothing. \\
\hline
\end{tabular}

Finally, a confusion matrix is computed using True Positive, True Negative, False Positive, and

False Negative scores and returned to the caller.

\section{TimeAverages.m}

TimeAverages. $m$ is responsible for reducing the full dataset into an average of each metric for the specified period. FirstDay and LastDay are optional parameters and default to 0 and 999,999,999 effectively using the entire data set.

The index is first advanced up to the first day in the set. When this has happened, the index into the dataset is advanced one by one until one "period" of data is identified. The mean of this data is computed, the index, startTime, and endTime variables are reset, and the process is repeated. 


\section{WindowScan.m}

This function computes the velocity of the normalized metric data. The data is normalized to the Minimum Detectable Change (MDC) computed over the first 30 days. This function first computes the baseline of the metric over the first "baselineWidth" days (typically 30). For each day, the departure from the baseline is computed using the average over 7 days starting from the current day. Finally, for this pass, the MDC departure from the baseline is computed by simply dividing the departure from the baseline by the MDC value over the initial "baslineWidth" days.

On the second pass, the change in average value from the "avgWindow" prior to the current day and the average value from the "avgWindow" following the current day is calculated. The MDC departure is also computed using the MDC computed over the first part of this window (today - avgWindow). Note that the code loosely refers to this as slope, though this is not the slope (though it is proportional to it). 


\section{References}

[1] S. R. Lord, J. A. Ward, P. Williams, and K. J. Anstey, "An epidemiological study of falls in older community-dwelling women: the Randwick falls and fractures study," Aust. J. Public Health, vol. 17, no. 3, pp. 240-245, 1993.

[2] P. Kannus et al., "Fall-induced injuries and deaths among older adults," Jama, vol. 281, no. 20, pp. 1895-1899, 1999.

[3] P. Scuffham, S. Chaplin, and R. Legood, "Incidence and costs of unintentional falls in older people in the United Kingdom," J. Epidemiol. Community Health, vol. 57, no. 9, pp. 740744, 2003.

[4] R. W. SATTIN et al., "THE INCIDENCE OF FALL INJURY EVENTS AMONG THE ELDERLY IN A DEFINED POPULATION," Am. J. Epidemiol., vol. 131, no. 6, pp. 1028-1037, Jun. 1990.

[5] J. A. Stevens, P. S. Corso, E. A. Finkelstein, and T. R. Miller, "The costs of fatal and non-fatal falls among older adults," Inj. Prev., vol. 12, no. 5, pp. 290-295, Oct. 2006.

[6] Nevitt MC, Cummings SR, Kidd S, and Black D, "Risk factors for recurrent nonsyncopal falls: A prospective study," JAMA, vol. 261, no. 18, pp. 2663-2668, May 1989.

[7] J. Howland, E. W. Peterson, W. C. Levin, L. Fried, D. Pordon, and S. Bak, "Fear of Falling among the Community-Dwelling Elderly," J. Aging Health, vol. 5, no. 2, pp. 229-243, May 1993.

[8] E. Stone and M. Skubic, "Passive, In-Home Gait Measurement Using an Inexpensive Depth Camera: Initial Results," 2012.

[9] R. Wallace, C. Abbott, C. Gibson-Horn, and M. Skubic, "In-home measurement of the effect of strategically weighted vests on ambulation," in Engineering in Medicine and Biology Society (EMBC), 2013 35th Annual International Conference of the IEEE, 2013, pp. 949-952.

[10] R. Wallace, C. Abbott, C. Gibson-Horn, M. Rantz, and M. Skubic, "Metrics from in-home sensor data to assess gait change due to weighted vest therapy," Smart Health, Apr. 2017.

[11] R. Wallace, C. Abbott, and M. Skubic, "Exploring Clinical Correlations in Centroid-Based Gait Metrics from Depth Data Collected in the Home.," presented at the 11th EAI International Conference on Pervasive Computing Technologies for Healthcare, Barcelona, Spain, 2017.

[12] E. E. Stone and M. Skubic, "Unobtrusive, Continuous, In-Home Gait Measurement Using the Microsoft Kinect," IEEE Trans. Biomed. Eng., vol. 60, no. 10, pp. 2925-2932, Oct. 2013.

[13] J. M. Guralnik et al., "A Short Physical Performance Battery Assessing Lower Extremity Function: Association With Self-Reported Disability and Prediction of Mortality and Nursing Home Admission," J. Gerontol., vol. 49, no. 2, pp. M85-M94, Mar. 1994.

[14] J. M. Guralnik, L. Ferrucci, E. M. Simonsick, M. E. Salive, and R. B. Wallace, "Lowerextremity function in persons over the age of 70 years as a predictor of subsequent disability," N. Engl. J. Med., vol. 332, no. 9, pp. 556-561, Mar. 1995.

[15] A. Corsonello et al., "Prognostic Significance of the Short Physical Performance Battery in Older Patients Discharged from Acute Care Hospitals," Rejuvenation Res., vol. 15, no. 1, pp. 41-48, Feb. 2012.

[16] P. W. Duncan, D. K. Weiner, J. Chandler, and S. Studenski, "Functional Reach: A New Clinical Measure of Balance," J. Gerontol., vol. 45, no. 6, pp. M192-M197, Nov. 1990.

[17] A. L. Behrman, K. E. Light, S. M. Flynn, and M. T. Thigpen, "Is the functional reach test useful for identifying falls risk among individuals with Parkinson's disease?," Arch. Phys. Med. Rehabil., vol. 83, no. 4, pp. 538-542, Apr. 2002. 
[18] K. Berg, "Measuring balance in the elderly: preliminary development of an instrument," Physiother. Can., vol. 41, no. 6, pp. 304-311, Jan. 1989.

[19] B. Ko, W.-D. SI, W. Ji, and M. B, "Measuring balance in the elderly: validation of an instrument.," Can. J. Public Health Rev. Can. Sante Publique, vol. 83 Suppl 2, pp. S7-11, Dec. 1991.

[20] E. A. Hurvitz, J. K. Richardson, R. A. Werner, A. M. Ruhl, and M. R. Dixon, "Unipedal stance testing as an indicator of fall risk among older outpatients," Arch. Phys. Med. Rehabil., vol. 81, no. 5, pp. 587-591, May 2000.

[21] D. Podsiadlo and S. Richardson, "The timed 'Up \& Go': a test of basic functional mobility for frail elderly persons," J. Am. Geriatr. Soc., vol. 39, no. 2, p. 142-148, Feb. 1991.

[22] T. M. Steffen, T. A. Hacker, and L. Mollinger, "Age-and gender-related test performance in community-dwelling elderly people: Six-Minute Walk Test, Berg Balance Scale, Timed Up \& Go Test, and gait speeds," Phys. Ther., vol. 82, no. 2, pp. 128-137, 2002.

[23] M. T. Kristensen, C. Ekdahl, H. Kehlet, and T. Bandholm, "How Many Trials Are Needed to Achieve Performance Stability of the Timed Up \&amp; Go Test in Patients With Hip Fracture?," Arch. Phys. Med. Rehabil., vol. 91, no. 6, pp. 885-889, Jun. 2010.

[24] J. C. Wall, C. Bell, S. Campbell, and J. Davis, "The Timed Get-up-and-Go test revisited: measurement of the component tasks," J. Rehabil. Res. Dev., vol. 37, no. 1, 2000.

[25] R. W. Bohannon, "Reference Values for the Timed Up and Go Test: A Descriptive MetaAnalysis," J. Geriatr. Phys. Ther., vol. 29, no. 2, pp. 64-68, 2006.

[26] S. Morris, M. E. Morris, and R. lansek, "Reliability of measurements obtained with the Timed 'Up \& Go' test in people with Parkinson disease," Phys. Ther., vol. 81, no. 2, pp. 810-818, 2001.

[27] J. Montes, B. Cheng, B. Diamond, C. Doorish, H. Mitsumoto, and P. H. Gordon, "The Timed Up and Go test: Predicting falls in ALS," Amyotroph. Lateral Scler., vol. 8, no. 5, pp. 292295, Jan. 2007.

[28] C. Walker, B. J. Brouwer, and E. G. Culham, "Use of visual feedback in retraining balance following acute stroke," Phys. Ther., vol. 80, no. 9, pp. 886-895, 2000.

[29] C. M. Arnold and R. A. Faulkner, "The history of falls and the association of the timed up and go test to falls and near-falls in older adults with hip osteoarthritis," BMC Geriatr., vol. 7, no. 1, p. 17, 2007.

[30] M. T. Kristensen, N. B. Foss, and H. Kehlet, "Factors with independent influence on the 'timed up and go' test in patients with hip fracture," Physiother. Res. Int., vol. 14, no. 1, pp. 30-41, Mar. 2009.

[31] R. W. Bohannon, "Comfortable and maximum walking speed of adults aged 20-79 years: reference values and determinants," Age Ageing, vol. 26, no. 1, pp. 15-19, 1997.

[32] H.-K. Kuo, S. G. Leveille, Y.-H. Yu, and W. P. Milberg, "Cognitive function, habitual gait speed, and late-life disability in the National Health and Nutrition Examination Survey (NHANES) 1999-2002," Gerontology, vol. 53, no. 2, pp. 102-110, 2006.

[33] X. Li et al., "Validity of an Exercise Test Based on Habitual Gait Speed in Mobility-Limited Older Adults," Arch. Phys. Med. Rehabil., vol. 93, no. 2, pp. 344-350, Feb. 2012.

[34] C. Gibson-Horn, "Balance-Based Torso-Weighting in a Patient with Ataxia and Multiple Sclerosis: A Case Report:," J. Neurol. Phys. Ther., vol. 32, no. 3, pp. 139-146, Sep. 2008.

[35] G. L. Widener, D. D. Allen, and C. Gibson-Horn, "Balance-Based Torso-Weighting May Enhance Balance in Persons With Multiple Sclerosis: Preliminary Evidence," Arch. Phys. Med. Rehabil., vol. 90, no. 4, pp. 602-609, Apr. 2009. 
[36] G. L. Widener, D. D. Allen, and C. Gibson-Horn, "Randomized Clinical Trial of Balance-Based Torso Weighting for Improving Upright Mobility in People with Multiple Sclerosis," Neurorehabil. Neural Repair, vol. 23, no. 8, pp. 784-791, Oct. 2009.

[37] A. Gorgas, G. Widener, C. Gibson-Horn, and D. D. Allen, Gait Parameters with and without Balance-Based Torso-Weighting in people with Multiple Sclerosis. 2011.

[38] A.-M. Gorgas, G. L. Widener, C. Gibson-Horn, and D. D. Allen, "Gait Changes with BalanceBased Torso-Weighting in People with Multiple Sclerosis: Balance-Based Torso-Weighting in MS," Physiother. Res. Int., vol. 20, no. 1, pp. 45-53, Mar. 2015.

[39] D. Giansanti, G. Maccioni, S. Cesinaro, F. Benvenuti, and V. Macellari, "Assessment of fallrisk by means of a neural network based on parameters assessed by a wearable device during posturography," Med. Eng. Phys., vol. 30, no. 3, pp. 367-372, Apr. 2008.

[40] S. Bamberg, A. Y. Benbasat, D. M. Scarborough, D. E. Krebs, and J. A. Paradiso, "Gait Analysis Using a Shoe-Integrated Wireless Sensor System," IEEE Trans. Inf. Technol. Biomed., vol. 12, no. 4, pp. 413-423, Jul. 2008.

[41] M. Marschollek, K.-H. Wolf, M. Gietzelt, G. Nemitz, H. Meyer Zu Schwabedissen, and R. Haux, "Assessing elderly persons' fall risk using spectral analysis on accelerometric data-a clinical evaluation study," in Engineering in Medicine and Biology Society, 2008. EMBS 2008. 30th Annual International Conference of the IEEE, 2008, pp. 3682-3685.

[42] P. Boissy, S. Choquette, M. Hamel, and N. Noury, "User-Based Motion Sensing and Fuzzy Logic for Automated Fall Detection in Older Adults," Telemed. E-Health, vol. 13, no. 6, pp. 683-694, Dec. 2007.

[43] W. Tao, T. Liu, R. Zheng, and H. Feng, "Gait Analysis Using Wearable Sensors," Sensors, vol. 12, no. 12, pp. 2255-2283, Feb. 2012.

[44] E. Porter, "Wearing and using personal emergency respone system buttons," J. Gerontol. Nurs., vol. 31, no. 10, p. 26-33, Oct. 2005.

[45] F. Wang, M. Skubic, C. Abbott, and J. M. Keller, "Body sway measurement for fall risk assessment using inexpensive webcams," in Engineering in Medicine and Biology Society (EMBC), 2010 Annual International Conference of the IEEE, 2010, pp. 2225-2229.

[46] F. Wang, "Motion analysis for in-home gait and balance assessment using inexpensive video sensors," University of Missouri-Columbia, 2011.

[47] D. Anderson, J. M. Keller, M. Skubic, X. Chen, and Z. He, "Recognizing falls from silhouettes," in Engineering in Medicine and Biology Society, 2006. EMBS'06. 28th Annual International Conference of the IEEE, 2006, pp. 6388-6391.

[48] E. E. Stone and M. Skubic, "Silhouette classification using pixel and voxel features for improved elder monitoring in dynamic environments," in Pervasive Computing and Communications Workshops (PERCOM Workshops), 2011 IEEE International Conference on, 2011, pp. 655-661.

[49] F. Wang, M. Skubic, C. Abbott, and J. M. Keller, "Quantitative analysis of 180 degree turns for fall risk assessment using video sensors," in Engineering in Medicine and Biology Society, EMBC, 2011 Annual International Conference of the IEEE, 2011, pp. 7606-7609.

[50] P. E. Cuddihy et al., "Radar walking speed measurements of seniors in their apartments: Technology for fall prevention," in Engineering in Medicine and Biology Society (EMBC), 2012 Annual International Conference of the IEEE, 2012, pp. 260-263.

[51] L. Liu, M. Popescu, K. C. Ho, M. Skubic, and M. Rantz, "Doppler radar sensor positioning in a fall detection system," in Engineering in Medicine and Biology Society (EMBC), 2012 Annual International Conference of the IEEE, 2012, pp. 256-259. 
[52] T. Yardibi et al., "Gait characterization via pulse-Doppler radar," in Pervasive Computing and Communications Workshops (PERCOM Workshops), 2011 IEEE International Conference on, 2011, pp. 662-667.

[53] C. E. Phillips et al., "Radar walk detection in the apartments of elderly," in Engineering in Medicine and Biology Society (EMBC), 2012 Annual International Conference of the IEEE, 2012, pp. 5863-5866.

[54] Y. Li, Z. Zeng, M. Popescu, and K. C. Ho, "Acoustic fall detection using a circular microphone array," in Engineering in Medicine and Biology Society (EMBC), 2010 Annual International Conference of the IEEE, 2010, pp. 2242-2245.

[55] M. Popescu and A. Mahnot, "Acoustic fall detection using one-class classifiers," in Engineering in Medicine and Biology Society, 2009. EMBC 2009. Annual International Conference of the IEEE, 2009, pp. 3505-3508.

[56] M. Popescu, Y. Li, M. Skubic, and M. Rantz, "An acoustic fall detector system that uses sound height information to reduce the false alarm rate," in Engineering in Medicine and Biology Society, 2008. EMBS 2008. 30th Annual International Conference of the IEEE, 2008, pp. 4628-4631.

[57] C.-Y. Hsu, Y. Liu, Z. Kabelac, R. Hristov, D. Katabi, and C. Liu, "Extracting Gait Velocity and Stride Length from Surrounding Radio Signals," presented at the ACM CHI, Denver, CO, 2017, pp. 2116-2126.

[58] R. Khusainov, D. Azzi, I. E. Achumba, and S. D. Bersch, "Real-Time Human Ambulation, Activity, and Physiological Monitoring: Taxonomy of Issues, Techniques, Applications, Challenges and Limitations," Sensors, vol. 13, no. 10, pp. 12852-12902, Sep. 2013.

[59] W. Wang, A. X. Liu, and M. Shahzad, "Gait recognition using wifi signals," 2016, pp. 363373.

[60] S. Piérard, S. Azrour, R. Phan-Ba, and M. Van Droogenbroeck, "GAIMS: A Reliable NonIntrusive Gait Measuring System." [Online]. Available: https://ercimnews.ercim.eu/en95/special/gaims-a-reliable-non-intrusive-gait-measuring-system. [Accessed: 27-Oct-2017].

[61] T. Pallejà, M. Teixidó, M. Tresanchez, and J. Palacín, "Measuring Gait Using a Ground Laser Range Sensor," Sensors, vol. 9, no. 11, pp. 9133-9146, Nov. 2009.

[62] M. Popescu, B. Hotrabhavananda, M. Moore, and M. Skubic, "VAMPIR - An Automatic Fall Detection System Using a Vertical PIR Sensor Array," 2012.

[63] M. Pavel et al., "Continuous assessment of gait velocity in Parkinson's disease from unobtrusive measurements," in Neural Engineering, 2007. CNE'07. 3rd International IEEE/EMBS Conference on, 2007, pp. 700-703.

[64] R. Rana, D. Austin, P. G. Jacobs, M. Karunanithi, and J. Kaye, "Gait Velocity Estimation using time interleaved between Consecutive Passive IR Sensor Activations," ArXiv13104880 Cs, Oct. 2013.

[65] E. E. Stone and M. Skubic, "Capturing habitual, in-home gait parameter trends using an inexpensive depth camera," in Engineering in Medicine and Biology Society (EMBC), 2012 Annual International Conference of the IEEE, 2012, pp. 5106-5109.

[66] E. Stone and M. Skubic, "Evaluation of an inexpensive depth camera for in-home gait assessment," J. Ambient Intell. Smart Environ., vol. 3, no. 4, pp. 349-361, 2011.

[67] B. Caby, S. Kieffer, M. de Saint Hubert, G. Cremer, and B. Macq, "Feature extraction and selection for objective gait analysis and fall risk assessment by accelerometry," Biomed. Eng. Online, vol. 10, no. 1, p. 1, 2011. 
[68] E. Stone, M. Skubic, M. Rantz, C. Abbott, and S. Miller, "Average in-home gait speed: Investigation of a new metric for mobility and fall risk assessment of elders," Gait Posture, vol. 41, no. 1, pp. 57-62, Jan. 2015.

[69] F. Wang, M. Skubic, M. Rantz, and P. E. Cuddihy, "Quantitative Gait Measurement With Pulse-Doppler Radar for Passive In-Home Gait Assessment," IEEE Trans. Biomed. Eng., vol. 61, no. 9, pp. 2434-2443, Sep. 2014.

[70] R. C. González, A. M. López, J. Rodriguez-Uría, D. Álvarez, and J. C. Alvarez, "Real-time gait event detection for normal subjects from lower trunk accelerations," Gait Posture, vol. 31, no. 3, pp. 322-325, Mar. 2010.

[71] S. D. Din, A. Godfrey, and L. Rochester, "Validation of an Accelerometer to Quantify a Comprehensive Battery of Gait Characteristics in Healthy Older Adults and Parkinson \#x0027;s Disease: Toward Clinical and at Home Use," IEEE J. Biomed. Health Inform., vol. 20, no. 3, pp. 838-847, May 2016.

[72] J. McCamley, M. Donati, E. Grimpampi, and C. Mazzà, "An enhanced estimate of initial contact and final contact instants of time using lower trunk inertial sensor data," Gait Posture, vol. 36, no. 2, pp. 316-318, Jun. 2012.

[73] K. S. van Schooten et al., "Daily-life gait quality as predictor of falls in older people: a 1year prospective cohort study," PLoS One, vol. 11, no. 7, p. e0158623, 2016.

[74] N. Carbonaro, F. Lorussi, and A. Tognetti, "Assessment of a Smart Sensing Shoe for Gait Phase Detection in Level Walking," Electronics, vol. 5, no. 4, p. 78, Nov. 2016.

[75] A. L. McDonough, M. Batavia, F. C. Chen, S. Kwon, and J. Ziai, "The validity and reliability of the GAITRite system's measurements: A preliminary evaluation," Arch. Phys. Med. Rehabil., vol. 82, no. 3, pp. 419-425, Mar. 2001.

[76] S.-G. Miaou, P.-H. Sung, and C.-Y. Huang, "A customized human fall detection system using omni-camera images and personal information," in Distributed Diagnosis and Home Healthcare, 2006. D2H2. 1st Transdisciplinary Conference on, 2006, pp. 39-42.

[77] C. Altuntas, F. Turkmen, A. Ucar, and Y. A. Akgul, "Measurement and Analysis of Gait by Using a Time-of-Flight Camera," ISPRS - Int. Arch. Photogramm. Remote Sens. Spat. Inf. Sci., vol. XLI-B3, pp. 459-464, Jun. 2016.

[78] A. Ejupi, Y. J. Gschwind, M. Brodie, W. L. Zagler, S. R. Lord, and K. Delbaere, "Kinect-based choice reaching and stepping reaction time tests for clinical and in-home assessment of fall risk in older people: a prospective study," Eur. Rev. Aging Phys. Act., vol. 13, no. 1, Dec. 2016.

[79] S. Lord and R. Fitzpatrick, "Choice stepping reaction time: a composite measure of falls risk in older people.," J Gerontol Biol Sci Med Sci, vol. 56, no. 10, pp. M627-32, Oct. 2001.

[80] K. J. Chun, D. Lim, C. Kim, H. Jung, and D. Jung, "Use of the Microsoft Kinect system to characterize balance ability during balance training," Clin. Interv. Aging, p. 1077, Jun. 2015.

[81] E. Cippitelli, S. Gasparrini, S. Spinsante, and E. Gambi, "Kinect as a Tool for Gait Analysis: Validation of a Real-Time Joint Extraction AlgorithmWorking in Side View," Sensors, vol. 15, no. 1, pp. 1417-1434, Jan. 2015.

[82] B. Müller, W. Ilg, M. A. Giese, and N. Ludolph, "Validation of enhanced kinect sensor based motion capturing for gait assessment," PloS One, vol. 12, no. 4, p. e0175813, 2017.

[83] T.-N. Nguyen, H.-H. Huynh, and J. Meunier, "Skeleton-Based Abnormal Gait Detection," Sensors, vol. 16, no. 11, p. 1792, Oct. 2016.

[84] A. Pfister, A. M. West, S. Bronner, and J. A. Noah, "Comparative abilities of Microsoft Kinect and Vicon 3D motion capture for gait analysis," J. Med. Eng. Technol., vol. 38, no. 5, pp. 274-280, 2014. 
[85] B. Galna, G. Barry, D. Jackson, D. Mhiripiri, P. Olivier, and L. Rochester, "Accuracy of the Microsoft Kinect sensor for measuring movement in people with Parkinson's disease," Gait Posture, vol. 39, no. 4, pp. 1062-1068, Apr. 2014.

[86] X. Xu, R. W. McGorry, L.-S. Chou, J. Lin, and C. Chang, "Accuracy of the Microsoft Kinect" for measuring gait parameters during treadmill walking," Gait Posture, vol. 42, no. 2, pp. 145-151, Jul. 2015.

[87] M. Gabel, R. Gilad-Bachrach, E. Renshaw, and A. Schuster, "Full body gait analysis with Kinect," in Engineering in Medicine and Biology Society (EMBC), 2012 Annual International Conference of the IEEE, 2012, pp. 1964-1967.

[88] R. A. Clark et al., "Instrumenting gait assessment using the Kinect in people living with stroke: reliability and association with balance tests," J. NeuroEngineering Rehabil., vol. 12, no. 1, p. 15, 2015.

[89] E. Dolatabadi, B. Taati, and A. Mihailidis, "Concurrent validity of the Microsoft Kinect for Windows v2 for measuring spatiotemporal gait parameters," Med. Eng. Phys., vol. 38, no. 9, pp. 952-958.

[90] A. Staranowicz, G. R. Brown, and G.-L. Mariottini, "Evaluating the accuracy of a mobile Kinect-based gait-monitoring system for fall prediction," in Proceedings of the 6th International Conference on PErvasive Technologies Related to Assistive Environments, 2013, p. 57.

[91] R. Krishnapuram and J. Keller, "A Possibilistic Approach to Clustering," IEEE Trans. Fuzzy Syst., vol. 1, no. 2, pp. 98-110, May 1993.

[92] N. R. Pal, K. Pal, J. M. Keller, and J. C. Bezdek, "A possibilistic fuzzy c-means clustering algorithm," IEEE Trans. Fuzzy Syst., vol. 13, no. 4, pp. 517-530, Aug. 2005.

[93] S. Wang, "Change Detetction for Eldercare Using Passive Sensing," Doctoral Dissertation, University of Missouri-Columbia, 2011.

[94] "Costs of Falls Among Older Adults | Home and Recreational Safety | CDC Injury Center." [Online]. Available: https://www.cdc.gov/homeandrecreationalsafety/falls/fallcost.html. [Accessed: 13-Mar-2017].

[95] J. M. Guralnik, L. Ferrucci, E. M. Simonsick, M. E. Salive, and R. B. Wallace, "Lowerextremity function in persons over the age of 70 years as a predictor of subsequent disability," N. Engl. J. Med., vol. 332, no. 9, pp. 556-562, 1995.

[96] N. Veronese et al., "Association Between Short Physical Performance Battery and Falls in Older People: The Progetto Veneto Anziani Study," Rejuvenation Res., vol. 17, no. 3, pp. 276-284, Jun. 2014.

[97] B. A. Springer, R. Marin, T. Cyhan, H. Roberts, and N. W. Gill, "Normative values for the unipedal stance test with eyes open and closed," J. Geriatr. Phys. Ther., vol. 30, no. 1, pp. 8-15, 2007.

[98] C. M. Hunt, G. Widener, and D. D. Allen, "Variability in Postural Control With and Without Balance-Based Torso- Weighting in People With Multiple Sclerosis and Healthy Controls," Phys. Ther., vol. 94, no. 10, pp. 1489-1498, Oct. 2014.

[99] F. Lin, W. Aosen, Z. Yan, M. R. Tomita, and X. Wenyao, "Smart Insole: a Wearable Sensor Device for Unobtrusive Gait Monitoring in Daily Life," IEEE Trans. Ind. Inform. TII, vol. 12, no. 6, pp. 2281-2291, Dec. 2016.

[100] G. Demiris et al., "Older adults' attitudes towards and perceptions of 'smart home' technologies: a pilot study," Inform. Health Soc. Care, vol. 29, no. 2, pp. 87-94, Jan. 2004.

[101] G. Demiris et al., "Smart home sensors for the elderly: a model for participatory formative evaluation," Hum.-Comput. Interact., vol. 6, p. 7, 2006. 
[102] J. Courtney and A. M. de Paor, "A Monocular Marker-Free Gait Measurement System," IEEE Trans. Neural Syst. Rehabil. Eng., vol. 18, no. 4, pp. 453-460, Aug. 2010.

[103] Fang Wang, E. Stone, M. Skubic, J. M. Keller, C. Abbott, and M. Rantz, "Toward a Passive Low-Cost In-Home Gait Assessment System for Older Adults," IEEE J. Biomed. Health Inform., vol. 17, no. 2, pp. 346-355, Mar. 2013.

[104] R. Chavez-Romero, A. Cardenas, J. M. Rendon-Mancha, K. M. Vernaza, and D. Piovesan, "Inexpensive Vision-Based System for the Direct Measurement of Ankle Stiffness During Quiet Standing," J. Med. Devices, vol. 9, no. 4, p. 041011, 2015.

[105] E. E. Stone and M. Skubic, "Unobtrusive, Continuous, In-Home Gait Measurement Using the Microsoft Kinect," IEEE Trans. Biomed. Eng., vol. 60, no. 10, pp. 2925-2932, Oct. 2013.

[106] Y.-J. Chang, S.-F. Chen, and J.-D. Huang, "A Kinect-based system for physical rehabilitation: A pilot study for young adults with motor disabilities," Res. Dev. Disabil., vol. 32, no. 6, pp. 2566-2570, Nov. 2011.

[107] T. Dutta, "Evaluation of the Kinect ${ }^{\mathrm{TM}}$ sensor for 3-D kinematic measurement in the workplace," Appl. Ergon., vol. 43, no. 4, pp. 645-649, Jul. 2012.

[108] R. A. Clark, Y.-H. Pua, A. L. Bryant, and M. A. Hunt, "Validity of the Microsoft Kinect for providing lateral trunk lean feedback during gait retraining," Gait Posture, vol. 38, no. 4, pp. 1064-1066, Sep. 2013.

[109] Y. Angad, S. Shandilya, and N. Kumar, "Implementation of Affordable Joint Angle Measurement for Gait Analysis using Kinect Image Sensor."

[110] E. E. Stone and M. Skubic, "Passive in-home measurement of stride-to-stride gait variability comparing vision and Kinect sensing," in Engineering in Medicine and Biology Society, EMBC, 2011 Annual International Conference of the IEEE, 2011, pp. 6491-6494.

[111] S. Arafat and M. Skubic, "Combined Uncertainty Model for Best Wavelet Selection," in Proceedings of the IEEE 2003 International Conference on Fuzzy Systems, St. Louis, MO, 2003.

[112] A. Deluca and S. Termini, "A Definition of Non-Probabilistic Entropy in the Setting of Fuzzy Set Theory," Inf. Control, vol. 20, pp. 301-302, 1972.

[113] N. R. Pal and S. K. Pal, "Object-background segmentation using new definitions of entropy," Comput. Digit. Tech. IEE Proc. E, vol. 136, no. 4, pp. 284-295, 1989.

[114] N. R. Pal and J. C. Bezdek, "Measuring fuzzy uncertainty," Fuzzy Syst. IEEE Trans. On, vol. 2, no. 2, pp. 107-118, 1994.

[115] J. Spooner, "NWQEP NOTES," The NCSU Water Quality Group Newsletter, pp. 1-11, Dec2011.

[116] M. M. Mukaka, "A guide to appropriate use of correlation coefficient in medical research," Malawi Med. J., vol. 24, no. 3, pp. 69-71, 2012.

[117] K. Pearson, "On lines and planes of closest fit to systems of points in space.," Philos. Mag., vol. 2, pp. 559-572, 1901.

[118] T. C. Havens and J. C. Bezdek, "An efficient formulation of the improved visual assessment of cluster tendency (iVAT) algorithm," IEEE Trans. Knowl. Data Eng., vol. 24, no. 5, pp. 813822, 2012.

[119] N. R. Pal, K. Pal, J. M. Keller, and J. C. Bezdek, "A possibilistic fuzzy c-means clustering algorithm," IEEE Trans. Fuzzy Syst., vol. 13, no. 4, pp. 517-530, Aug. 2005.

[120] S. Wang, "Change Detetction for Eldercare Using Passive Sensing," Doctoral Dissertation, University of Missouri-Columbia, 2011.

[121] M. Popescu and A. Mahnot, "Acoustic fall detection using one-class classifiers," in Engineering in Medicine and Biology Society, 2009. EMBC 2009. Annual International Conference of the IEEE, 2009, pp. 3505-3508. 


\section{VITA}

Robert Wallace received the B.S. and M.S. degrees in Biomedical Engineering from Boston University in 1987 and 1991 respectively. His M.S. thesis was titled Development of a Software Package for Multiple Unit Analysis of Neurophysiological Data and involved the creation of a software package, in FORTRAN IV and FORTRAN 77, for conducting multi-unit analysis, using autocorrelation and cross-correlation in particular, of neural action potential trains recorded in response to sound stimulus in gerbil.

From 1991 to 1999, he worked in increasing levels of responsibility as a software engineer for Critikon, Inc., a division of Johnson \& Johnson, designing and developing software for medical patient monitors. In 1997, while at Critikon, Robert started work on an M.S. degree in Engineering Management which he completed in 2001. In 1999, Robert accepted a position with Baxter Healthcare developing software for hemodialysis and later peritoneal dialysis devices. During this time, Robert also expanded his role from that of a technical contributor to include more managerial responsibilities, eventually working as a project manager. Robert left Baxter in 2009 as part of a facility closure.

In 2007, while at Baxter, Robert developed and began teaching a course in Embedded Systems Programming at Brandeis University. For several years, Robert taught both Embedded Systems Programming and Software Engineering for Brandeis. Later, Robert developed graduate courses in Advanced Software Engineering and Wearable Technology (with another professor). In 2010, Robert also began teaching as an Adjunct Professor at Columbia College in Information Technology. While at Columbia, Robert developed an undergraduate course in Software Engineering which he has been teaching since 2012. 
Also in 2010, Robert was accepted into the Computer Science PhD program at University of Missouri Columbia. During this time, he worked as a teaching assistant for several classes as well as a research assistant from 2010 to 2013 . From 2013 to 2014, Robert worked as a research software engineer for Washington University in St. Louis where he developed an on-line analysis portal allowing researchers to upload and process mass spectrometry data.

Working under the guidance of Dr. Marjorie Skubic, he completed his dissertation titled Development of Centroid Based Metrics to Provide Personalized Monitoring and Assessment of Gait in 2017. His areas of interest include eldercare technology, machine learning, neural networks, embedded software, hardware / software interfaces, and software engineering. 